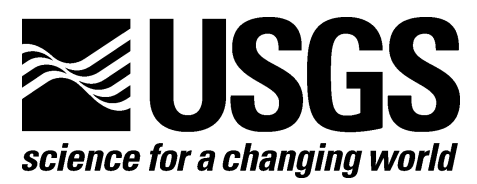

Prepared in cooperation with the U.S. Environmental Protection Agency

\title{
Assessment of 1-chloro-4-[2,2-dichloro-1-(4- chlorophenyl)ethenyl]benzene (DDE) Transformation Rates on the Palos Verdes Shelf, CA
}

By Robert P. Eganhouse and James Pontolillo

Open-File Report 2007-1362 


\section{U.S. Department of the Interior DIRK KEMPTHORNE, Secretary}

\section{U.S. Geological Survey \\ Mark D. Myers, Director}

\section{U.S. Geological Survey, Reston, Virginia: 2008}

For product and ordering information:

World Wide Web: http://www.usgs.gov/pubprod

Telephone: 1-888-ASK-USGS

For more information on the USGS - the Federal source for science about the Earth, its natural and living resources, natural hazards, and the environment:

World Wide Web: http://www.usgs.gov

Telephone: 1-888-ASK-USGS

Suggested citation:

Eganhouse, R.P., and Pontolillo, James, 2008, Assessment of 1-chloro-4-[2,2-dichloro-1-(4chlorophenyl)ethenyl]benzene (DDE) transformation rates on the Palos Verdes Shelf, CA: U.S. Geological Survey Open-File Report 2007-1362, 119 p., 7 appendices, available only online at http://pubs.usgs.gov/of/2007/1362.

Any use of trade, product, or firm names is for descriptive purposes only and does not imply endorsement by the U.S. Government.

Although this report is in the public domain, permission must be secured from the individual copyright owners to reproduce any copyrighted material contained within this report. 


\section{Contents}

Abstract

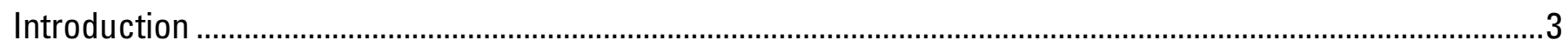

Impetus for this study

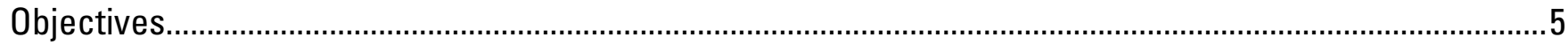

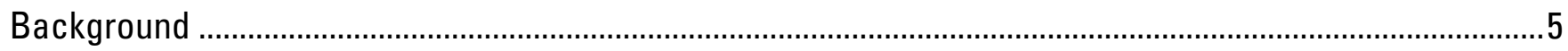

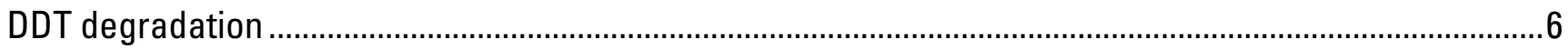

Previous work on the Palos Verdes Shelf .............................................................................................

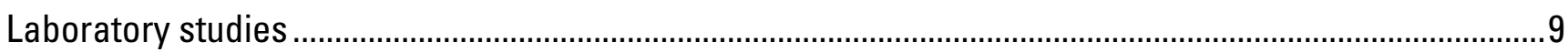

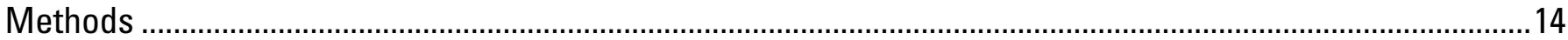

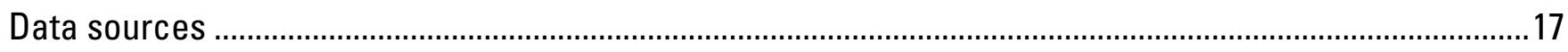

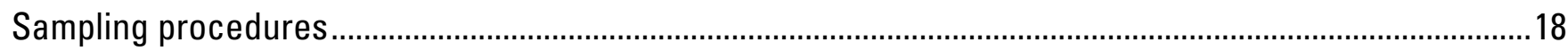

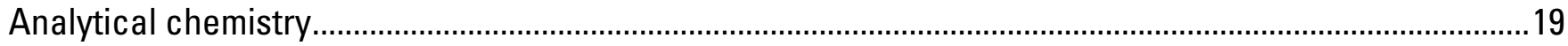

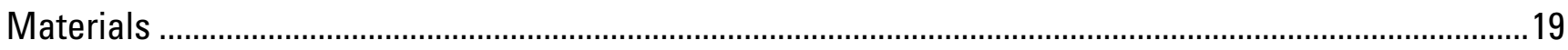

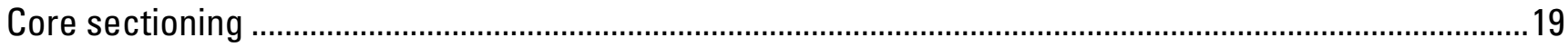

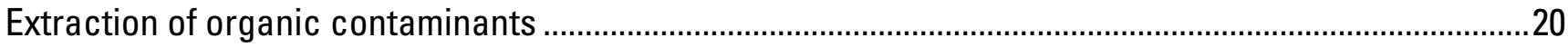

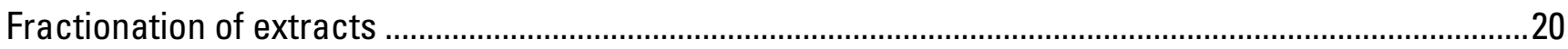

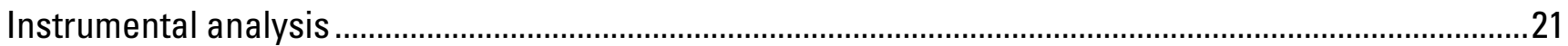

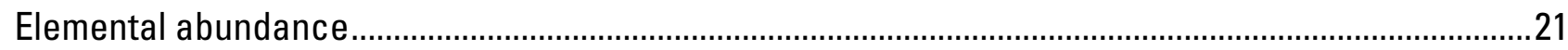

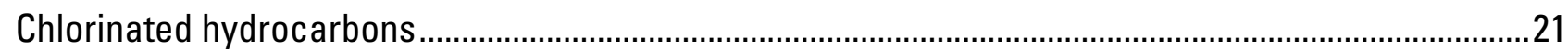

Long-chain alkylbenzenes ...............................................................................................................

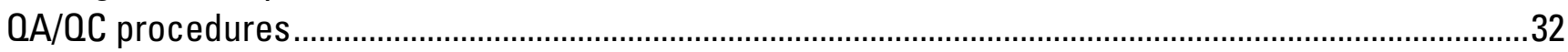

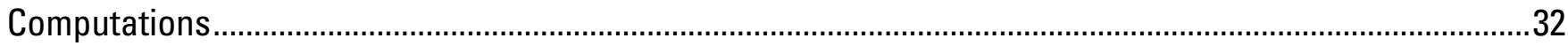

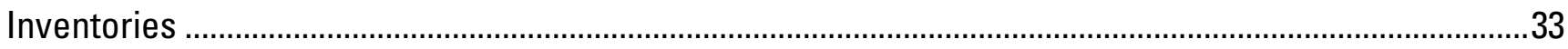

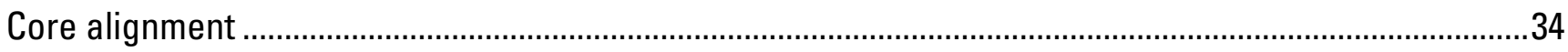

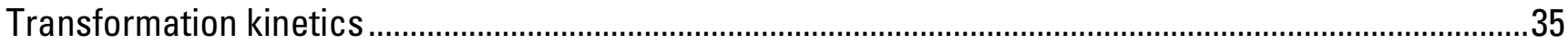

Modeling multi-step transformation kinetics..........................................................................................

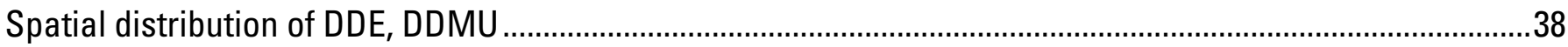

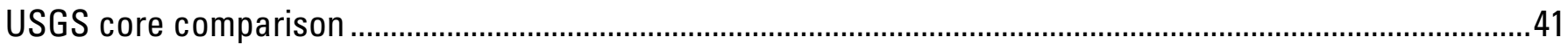

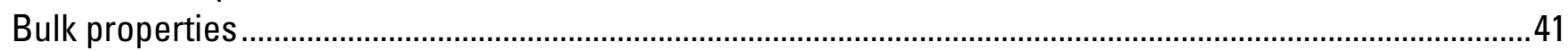

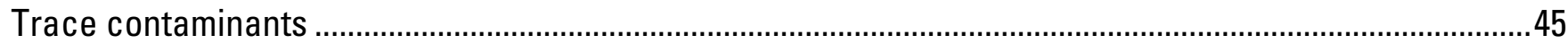

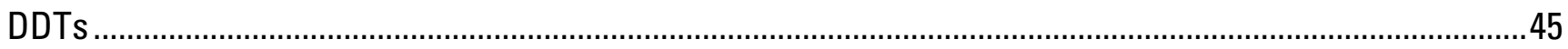

Polychlorinated biphenyls .................................................................................................................4

Long-chain alkylbenzenes..............................................................................................................

Transformation rates, pathways, products and kinetics.................................................................................53

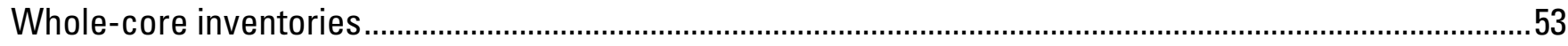

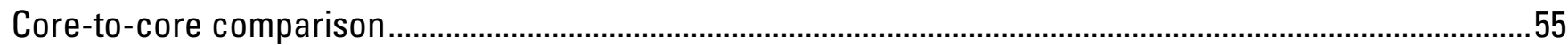

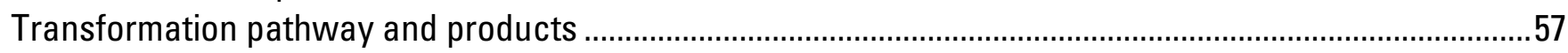

Environmental factors controlling DDE transformation rates .........................................................................58

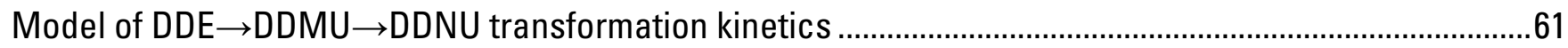

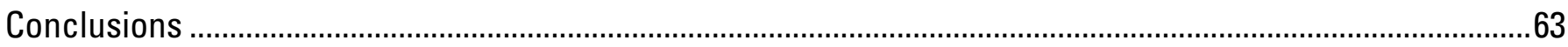

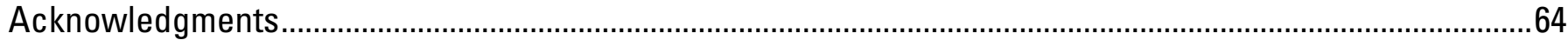

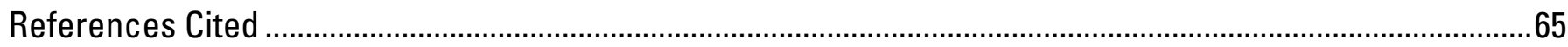


Appendices

1. Procedures for Preparation of Clean Reagents and Labware: Trace

Organics

(Available in a separate file)

2. Fractionation of Sediment Extracts for Determination of Trace Organics

3. Sectioning of Sediment Cores (Available in a separate file)

4. Extraction of Sediments for Determination of Trace Organics. (Available in a separate file)

5. Instrumental Analysis for the Long-Chain Alkylbenzenes (Available in a separate file)

6. Instrumental Analysis for Total Organic Carbon and Total Nitrogen in Sediments (Available in a separate file)

7. Instrumental Analysis for Chlorinated Hydrocarbons (Available in a separate file) (Available in a separate file)

\section{Figures}

1. Shaded relief maps showing $(A)$ Los Angeles, the Palos Verdes Peninsula and adjoining coastal bathymetry, and $(B)$ the Palos Verdes Shelf study site.

2. Graphs showing percent $p, p^{\prime}$-DDMU (relative to the sum, $p, p^{\prime}$-DDMU $+p, p^{\prime}-\mathrm{DDE}$ ) and vertical concentration ( $p, p^{\prime}$-DDMU $+p, p^{\prime}$-DDE) profiles in cores collected by the LACSD (Los Angeles County Sanitation Districts) at station 3C in 1991 and 2005.

3. Graphs showing percent $p, p^{\prime}$-DDMU (relative to the sum, $p, p^{\prime}-\mathrm{DDMU}+p, p^{\prime}-\mathrm{DDE}$ ) and vertical concentration $\left(p, p^{\prime}\right.$-DDMU $+p, p^{\prime}$-DDE) profiles in cores collected by the LACSD (Los Angeles County Sanitation Districts) at station 6C in 1991 and 2005.

4. Chart showing potential pathways for microbial degradation of DDT 76

5. Shaded relief maps showing sediment coring stations: (A) USGS-1992, (B) LACSD (Los Angeles

County Sanitation Districts), (C) and (D) SAIC (Science Applications International Corporation) 2004 (SU is a cell designation).

6. Shaded relief maps showing locations of cores collected by the USGS in 1992 and 2003 .........................79

7. Diagram showing extruder used for sectioning frozen sediment.

8. Figure showing gas chromatograms of $(A)$ DDT calibration standard solution, $(B)$ DDT F2 split of

30-32 cm section of sediment core 124B1-CH (2003), (C) PCB (polychorinated biphenyl) calibration standard solution, and (D) PCB F2 split of 30-32 cm section of sediment core 124B1-CH (2003)........81

9. Figure showing gas chromatograms and structures of $(A)$ linear alkylbenzenes (LABs), and $(B)$ tetrapropylene-based alkylbenzenes (TABs)

10. Figure showing total ion current (TIC) of (A) tetrapropylene-based alkylbenzene (TAB) calibration standard, and $(B)$ linear alkylbenzene (LAB) calibration standard, and mass fragmentograms for LCAB (long-chain alkylbenzene) F2 split of 30-32 cm section of 124B1-CH (2003) at (C) m/z=91, (D) $\mathrm{m} / \mathrm{z}=105,(E) \mathrm{m} / \mathrm{z}=119(\mathrm{~m} / \mathrm{z}=$ mass $/$ charge ratio).

11. Graph showing vertical concentration profiles of $p, p^{\prime}$-DDE in box core 147B3 (filled circles) and gravity core $179 \mathrm{G} 3$ (open circles) collected in 1992 at station 556 by the USGS.

12. Graphs showing core alignment curves based on Pearson correlation analyses of concentration/inventory compared to $(A)$ depth $(\mathrm{cm})$, and $(B)$ mass accumulation $\left(\mathrm{g} / \mathrm{cm}^{2}\right)$ offsets for cores 124B1-DDT (1992) and 124B1-CH (2003).

13. Graphs showing vertical concentration profiles of PCB $153(\mathrm{ng} / \mathrm{g}$ ) in cores 124B1-DDT (1992-blue lines) and 124B1-CH (2003-red lines) cores $(A)$ without depth offset, and $(B)$ with depth offset of 4 $\mathrm{g} / \mathrm{cm}^{2}$ 
14. Graphs showing cumulative inventory (CI) profiles for PCB 153 in cores 124B1-DDT (1992) and 124B1-CH (2003): $(A)$ without depth offset, and $(B)$ with depth offsets of 3 and $4 \mathrm{~g} / \mathrm{cm}^{2}$

15. Graphs showing percent $p, p^{\prime}$-DDMU (relative to the sum, $p, p^{\prime}$-DDMU $+p, p^{\prime}$-DDE; filled circles) and vertical concentration ( $p, p^{\prime}$-DDMU $+p, p^{\prime}$-DDE) profiles (solid gray lines) for cores collected by the USGS in 1992.

16. Graphs showing percent $p, p^{\prime}$-DDMU (relative to the sum, $p, p^{\prime}$-DDMU $+p, p^{\prime}$-DDE; filled circles) and vertical concentration ( $p, p^{\prime}$-DDMU $+p, p^{\prime}$-DDE) profiles (solid gray lines) for cores collected by the LACSD (Los Angeles County Sanitation Districts) at station 5C in 2005

17. Graphs showing percent $p, p^{\prime}$-DDMU (relative to the sum, $p, p^{\prime}$-DDMU $+p, p^{\prime}$-DDE; filled circles) and vertical concentration ( $p, p^{\prime}$-DDMU $+p, p^{\prime}$-DDE) profiles (solid gray lines) for cores collected by the LACSD (Los Angeles County Sanitation Districts; gravity) and SAIC (Science Applications International Corporation; piston) in 2004 at station A

18. Graphs showing percent $p, p^{\prime}$-DDMU (relative to the sum, $p, p^{\prime}$-DDMU + p, $p^{\prime}$-DDE; filled circles) and vertical concentration ( $p, p^{\prime}$-DDMU $+p, p^{\prime}$-DDE) profiles (solid gray lines) for cores collected by the LACSD (Los Angeles County Sanitation Districts; gravity) and SAIC (Science Applications International Corporation; piston) in 2004 at stations 7C and 7CB

19. Shaded relief maps showing percent $p, p^{\prime}$-DDMU in box cores collected by the USGS in 1992: $(A) 0-4$ $\mathrm{cm},(B)$ 0-8 cm, $(C)$ whole-core

20. Shaded relief maps showing percent $p, p^{\prime}$-DDMU in cores collected by the: (A) USGS-1992, (B) LACSD-

1991, (C) LACSD-2005.

21. Graphs showing vertical density profiles for 124B1 cores collected by the USGS in 1992 and 2003 near station 3C: $(A)$ bulk density, $(B)$ dry density.

22. Graphs showing vertical profiles of the ${ }^{*} \mathrm{C} 28 /$ hopane ratio in $(A)$ the $19813 \mathrm{C} 1$ core, and $(B)$ the 124B1-

DDT (1992) and 124B1-CH (2003) cores.

23. Graphs showing vertical concentration profiles of $(A)$ total organic carbon (TOC), and (B) total nitrogen

(TN) in sediment cores collected by the USGS near station 3C in 1992 and 2003.

24. Graphs showing vertical concentration profiles of total extractable organics (TEO) in sediment cores collected by the USGS near station 3C in 1992 and 2003

25. Graphs showing average composition of DDTs in (A) Montrose effluent, sewer samples, LACSD effluent, and sediments from the Palos Verdes Shelf (after Eganhouse and others, 2000), and $(B)$ cores collected by the USGS near station 3C in 1992 and 2003

26. Graphs showing vertical concentration profiles of three DDT compounds in cores collected by the USGS near station 3C in (A) 1992, and $(B) 2003$

27. Graphs showing distribution of PCBs by chlorination level in cores 124B1-DDT (1992) and 124B1-CH

(2003) along with distributions found in three common Aroclors

28. Graphs showing vertical concentration profiles of PCB congeners in cores collected by the USGS near station 3C in 1992 and 2003: $(A)$ sum of 65 PCB peaks (68 congeners) free of major interference $\left(\Sigma \mathrm{PCB}_{65}\right),(B)$ sum of 18 interference-free PCB congeners $\left(\Sigma \mathrm{PCB}_{18}\right)$, and $(C) \mathrm{PCB}$ congener $153 \ldots . . .101$

29. Graphs showing vertical profiles of molar abundance of chlorine/biphenyl in the 124B1-DDT (1992) and 124B1-CH (2003) cores: $(A)$ total chlorines/biphenyl, $(B)$ meta- + para-chlorines/biphenyl, and $(C)$ ortho-chlorines/biphenyl

30. Graphs showing $(A)$ total, meta- + para-, ortho-, meta- and para-chlorine abundances, and $(B)$ meta- + para-chlorine abundance versus ortho-chlorine abundances in 124B1 cores (average) and Aroclors based on 68 PCB congeners 
31. Graphs showing vertical profiles of $(A)$ meta- $\mathrm{Cl} /$ biphenyl abundance and $\Sigma \mathrm{PCB}_{65}$ concentration, $(B)$ para-Cl/biphenyl abundance, and (C) ortho-Cl/biphenyl abundance in cores 124B1-DDT (1992) and 124B1-CH (2003). 104

32. Graphs showing difference between molar composition of $\mathrm{PCB}$ congeners in sediments from cores 124B1-DDT (1992) and 124B1-CH (2003) at the bottom and tops of the cores. 105

33. Graphs showing percent molar composition of PCBs in cores (A) 124B1-DDT (1992), and (B) 124B1-CH (2003) at depths of maximum concentration $(30-32 \mathrm{~cm})$ along with $(C)$ plot of difference between the cores.

34. Graph showing synthetic alkylbenzene sulfonate surfactant consumption in the United States (1950-1992). 107

35. Graph showing $(A)$ alkyl chain length and $(B)$ dodecylbenzene isomer compositions of linear alkylbenzenes in sediment cores, LACSD effluent

36. Graph showing composition of TABs in 124B1 cores: $(A)$ average for all depths (error bars $=1$ standard deviation), and $(B)$ abundance in section of maximum PCB/DDT concentration $(30-32 \mathrm{~cm})$.

37. Graph showing vertical concentration profiles of long-chain alkylbenzenes in cores collected by the USGS near station 3C in 1992 and 2003: $(A)$ total linear alkylbenzenes $\left(\Sigma L^{2} B_{26}\right),(B) 6$ phenyldodecane $\left(6-C_{12}\right),(C)$ summed TAB analytes $\left(\Sigma T_{A B}{ }_{12}\right)$, and $(D)$ a persistent TAB (TAB3) .......110

38. Graph showing vertical profiles of $(A)$ first-order $p, p^{\prime}$-DDE transformation rate constant, $\lambda_{92: 033^{\prime}}$ computed from aligned core data, and $(B)$ concentration of $p, p^{\prime}$-DDE, and $(C) p, p^{\prime}$-DDMU in 1992, 2003 cores

39. Graph showing vertical concentration profiles of $(A) p, p^{\prime}$-DDE, $(B) p, p^{\prime}$-DDMU, $(C) p, p^{\prime}$-DDNU in cores collected by the USGS near station 3C in 1992 and 2003

40. Graph showing vertical profiles of $(A)$ summed molar inventories of $p, p^{\prime}-\mathrm{DDE}+p, p^{\prime}-\mathrm{DDMU}+p, p^{\prime}-\mathrm{DDNU}$, and $(B)$ percent difference in summed molar inventories of cores 124B1-DDT (1992) and 124B1-CH (2003). 113

41. Graph showing trajectories of first-order transformation for $p, p^{\prime}$-DDE, $p, p^{\prime}$-DDMU, and $p, p^{\prime}$-DDNU near station 3C: $(A) 1992$ to 2003, (B) 1992 to 2092 114

\section{Tables}

1. Summary of DDE dechlorination rate data from laboratory microcosm experiments. 14

2. Summary information for cores collected on the Palos Verdes Shelf that are discussed in this report....16

3. List of target analytes. .23

4. Ratings of PCB congener target analytes for potential interferences and inclusion in PCB metrics.........28

5. Whole-core inventories of bulk constituents and trace contaminants in cores collected by the USGS in 1992 and 2003 and by the Los Angeles County Sanitation Districts in 1991 and 2005.

6. Comparison of DDE transformation rates in sediments of the Palos Verdes Shelf with results of relevant microcosm studies. 


\section{Conversion Factors}

\begin{tabular}{|c|c|c|}
\hline Multiply & By & To obtain \\
\hline \multicolumn{3}{|c|}{ Length } \\
\hline inch (in.) & 2.54 & centimeter $(\mathrm{cm})$ \\
\hline inch (in.) & 25.4 & millimeter (mm) \\
\hline foot $(\mathrm{ft})$ & 0.3048 & meter $(\mathrm{m})$ \\
\hline mile (mi) & 1.609 & kilometer (km) \\
\hline \multicolumn{3}{|c|}{ Area } \\
\hline square foot $\left(\mathrm{ft}^{2}\right)$ & 929.0 & square centimeter $\left(\mathrm{cm}^{2}\right)$ \\
\hline square foot $\left(\mathrm{ft}^{2}\right)$ & 0.09290 & square meter $\left(\mathrm{m}^{2}\right)$ \\
\hline square mile $\left(\mathrm{mi}^{2}\right)$ & 2.590 & square kilometer $\left(\mathrm{km}^{2}\right)$ \\
\hline \multicolumn{3}{|c|}{ Volume } \\
\hline ounce, fluid (fl. oz) & 0.02957 & liter $(\mathrm{L})$ \\
\hline cubic inch $\left(\right.$ in $\left.^{3}\right)$ & 16.39 & cubic centimeter $\left(\mathrm{cm}^{3}\right)$ \\
\hline cubic inch $\left(\mathrm{in}^{3}\right)$ & 0.01639 & liter $(\mathrm{L})$ \\
\hline \multicolumn{3}{|c|}{ Mass } \\
\hline ounce, avoirdupois (oz) & 28.35 & gram $(\mathrm{g})$ \\
\hline pound, avoirdupois (lb) & 0.4536 & kilogram (kg) \\
\hline ton per day (ton/d) & 0.9072 & metric ton per day \\
\hline ton per year (ton/yr) & 0.9072 & metric ton per year \\
\hline
\end{tabular}

Temperature in degrees Celsius $\left({ }^{\circ} \mathrm{C}\right)$ may be converted to degrees Fahrenheit $\left({ }^{\circ} \mathrm{F}\right)$ as follows: ${ }^{\circ} \mathrm{F}=\left(1.8 \times^{\circ} \mathrm{C}\right)+32$

Temperature in degrees Fahrenheit $\left({ }^{\circ} \mathrm{F}\right)$ may be converted to degrees Celsius $\left({ }^{\circ} \mathrm{C}\right)$ as follows: ${ }^{\circ} \mathrm{C}=\left({ }^{\circ} \mathrm{F}-32\right) / 1.8$ 


\section{Units, Acronyms and Abbreviations}

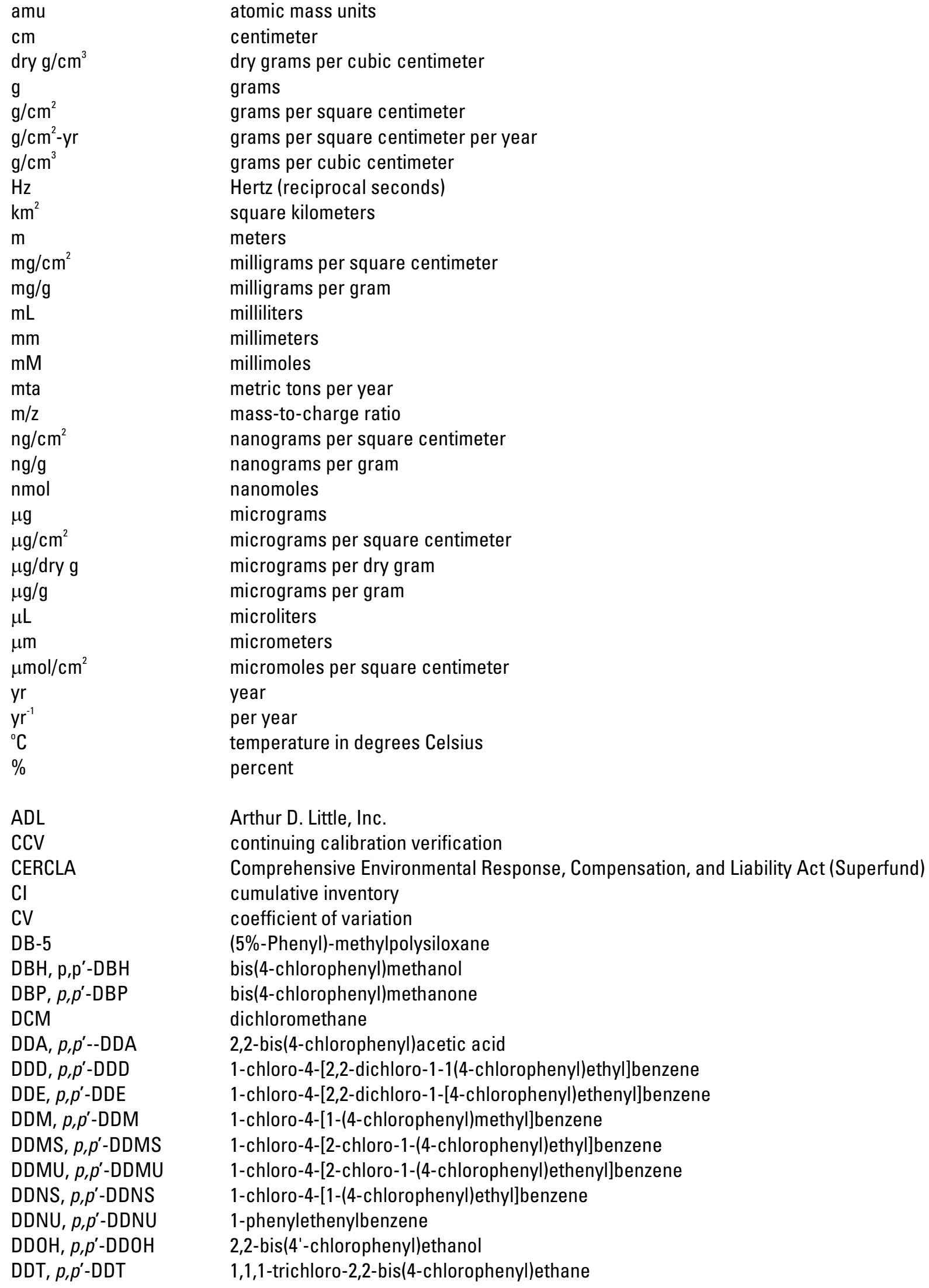

atomic mass units

centimeter

dry grams per cubic centimeter

grams

grams per square centimeter

grams per square centimeter per year

grams per cubic centimeter

Hertz (reciprocal seconds)

square kilometers

meters

milligrams per square centimeter

milligrams per gram

milliliters

millimeters

millimoles

metric tons per year

mass-to-charge ratio

nanograms per square centimeter

nanograms per gram

nanomoles

micrograms

micrograms per square centimeter

micrograms per dry gram

micrograms per gram

microliters

micrometers

micromoles per square centimeter

year

per year

temperature in degrees Celsius

percent

Arthur D. Little, Inc.

continuing calibration verification

Comprehensive Environmental Response, Compensation, and Liability Act (Superfund)

cumulative inventory

coefficient of variation

(5\%-Phenyl)-methylpolysiloxane

bis(4-chlorophenyl)methanol

bis(4-chlorophenyl)methanone

dichloromethane

2,2-bis(4-chlorophenyl)acetic acid

1-chloro-4-[2,2-dichloro-1-1(4-chlorophenyl)ethyl]benzene

1-chloro-4-[2,2-dichloro-1-[4-chlorophenyl)ethenyl]benzene

1-chloro-4-[1-(4-chlorophenyl)methyl]benzene

1-chloro-4-[2-chloro-1-(4-chlorophenyl)ethyl]benzene

1-chloro-4-[2-chloro-1-(4-chlorophenyl)ethenyl]benzene

1-chloro-4-[1-(4-chlorophenyl)ethyl]benzene

1-phenylethenylbenzene

2,2-bis(4'-chlorophenyl)ethanol

1,1,1-trichloro-2,2-bis(4-chlorophenyl)ethane 


\begin{tabular}{|c|c|}
\hline DGPS & differential geostationary positioning system \\
\hline $\mathrm{EC}-\mathrm{NCl}$ & electron capture negative chemical ionization \\
\hline EC-NCIMS & electron capture negative chemical ionization mass spectrometry \\
\hline El & electron impact \\
\hline El-HRGC/MS & electron impact high resolution gas chromatography/mass spectrometry \\
\hline GC & gas chromatography \\
\hline $\mathrm{GC} / \mathrm{ECD}$ & gas chromatography/electron capture detection \\
\hline GC/MS & gas chromatography/mass spectrometry \\
\hline GPS & geostationary positioning system \\
\hline HRGC/ECD & high resolution gas chromatography/electron capture detection \\
\hline HRGC/MS & high resolution gas chromatography/mass spectrometry \\
\hline i.d. & inner diameter \\
\hline I/E ratio & internal to external phenyldodecane isomer ratio (index of biodegradation) \\
\hline IS & internal quantitation standard \\
\hline JWPCP & Joint Water Pollution Control Plant \\
\hline LABs & linear alkylbenzenes \\
\hline LACSD & Los Angeles County Sanitation Districts \\
\hline LACSD-OMP & Los Angeles County Sanitation Districts Ocean Monitoring Program \\
\hline LAS & linear alkylbenzene sulfonates \\
\hline LCABs & long-chain alkylbenzenes \\
\hline MDLs & method detection limits \\
\hline $\mathrm{MeOH}$ & methanol \\
\hline Milli-QTM & ultrapure laboratory grade water filtered by reverse osmosis \\
\hline MPC & multi-point calibration \\
\hline $\mathrm{Na}_{2} \mathrm{SO}_{4}$ & sodium sulfate \\
\hline NEL & Naval Electronics Lab \\
\hline NIST & National Institute of Standards and Technology \\
\hline NOAA & National Oceanic and Atmospheric Administration \\
\hline o.d. & outer diameter \\
\hline PACS-1 & a marine sediment reference material \\
\hline $\mathrm{PAH}$ & polycyclic aromatic hydrocarbon \\
\hline PBL & Portuguese Bend Landslide \\
\hline РCB & polychlorinated biphenyl \\
\hline PVRP & Palos Verdes Remediation Project \\
\hline $\mathrm{QA} / \mathrm{QC}$ & quality assurance/quality control \\
\hline$r, r^{2}$ & correlation coefficient \\
\hline RPD & relative percent difference \\
\hline RS & recovery standard \\
\hline s.d. & standard deviation \\
\hline SAIC & Science Applications International Corporation \\
\hline SAIC-SDS & Science Applications International Corporation Sediment Displacement Study \\
\hline SCBNRDAP & Southern California Bight Natural Resources Damage Assessment Program \\
\hline SCCWRP & Southern California Coastal Water Research Project \\
\hline SOP & standard operating procedure \\
\hline SPC & single-point calibration \\
\hline SRM & standard reference material \\
\hline St. & station \\
\hline Std. & standard \\
\hline Std. dev. & standard deviation \\
\hline TABs & tetrapropylene-based alkylbenzenes \\
\hline TBS & tetrapropylene benzene sulfonate \\
\hline TC & total carbon \\
\hline TCMX & tetrachloro-meta-xylene \\
\hline TEAP & terminal electron accepting process \\
\hline
\end{tabular}


total extractable organics

total ion current

thin-layer chromatography

total nitrogen

total organic carbon

U.S. Army Corps of Engineers

U.S. Department of Justice

U. S. Environmental Protection Agency

U.S. Geological Survey

volume/volume basis

Woods Hole Group Environmental Laboratories

PC-based shipboard navigation system 


\title{
Assessment of 1-chloro-4-[2,2-dichloro-1-(4- chlorophenyl)ethenyl]benzene (DDE) Transformation Rates on the Palos Verdes Shelf, CA
}

\author{
By Robert P. Eganhouse and James Pontolillo
}

\begin{abstract}
Numerous investigations of the Palos Verdes Shelf near Los Angeles, California have been undertaken since the late 1960s, but few have focused on the biogeochemical fate of DDT and its transformation products (hereafter referred to as DDT). In 1972, one year after inputs of DDT to the LACSD (Los Angeles County Sanitation Districts) sewer system had ceased, the DDT composition of sediments on the Palos Verdes Shelf was dominated by $p, p$ '-DDE. Existing information suggests that this was the result of dehydrochlorination of $p$, $p$ '-DDT, which occurred shortly after discharge of DDTbearing wastes from the LACSD outfalls. The DDT composition of shelf sediments showed little change between 1972 and 1992 when, for the first time, $p, p$ '-DDMU was measured in the sediments as part of the Southern California Bight Natural Resource Damage Assessment Program (SCBNRDAP) in support of the Montrose case (United States of America, et al., vs. Montrose Chemical Corporation of California, et al.). The presence of $p$, $p$ '-DDMU in all sediment samples collected in 1992 and its increased relative abundance with depth in many cores suggested that reductive dechlorination of $p, p$ 'DDE was taking place. However, initial estimates of maximum first-order transformation rates near LACSD station $3 \mathrm{C}$, based on the assumption that $p, p$ '-DDMU was a dead-end product, were relatively low $\left(0.028 \mathrm{yr}^{-1}\right)$. Microcosm experiments published in 1998 demonstrated unequivocally that reductive dechlorination could be mediated by microorganisms present in Palos Verdes Shelf sediments. These experiments also showed that dechlorination rates varied spatially with higher rates in sediments collected farther from the outfalls (for example, LACSD station 3C); substantially higher rates were obtained under methanogenic (as opposed to sulfidogenic) conditions. The microcosm-based dechlorination rates determined for sediments from LACSD station 3C (sulfidogenic-0.20 $\mathrm{yr}^{-1}$; methanogenic- $0.99 \mathrm{yr}^{-1}$ ) were much higher than those estimated from the 1992 core analyses. The discrepancy between microcosm-based rates and those developed from field data is primarily attributable to differences between the conditions used in the laboratory experiments and those in the field. Subsequent to the first microcosm study, results of two additional laboratory investigations were released, both of which attempted to address these issues and provide insights into factors controlling the rate of reductive dechlorination of $p, p$ '-DDE in Palos Verdes Shelf sediments.

In 2005 the LACSD discovered that the abundance of $p, p$ '-DDMU in sediment cores had increased since the original 1992 study. Moreover, greater changes were seen at station 3C than at stations nearer the outfall system $(5 \mathrm{C}, 6 \mathrm{C})$. These observations prompted the present investigation, the goals of which were to synthesize available information concerning the degradation of DDT in Palos Verdes Shelf sediments and to develop estimates of in situ p,p'-DDE transformation rates. In 2003 the USGS collected a core near LACSD station 3C using the same navigational and sampling procedures as
\end{abstract}


those employed in the 1992 study. This core was analyzed by the USGS in 2006 for eight DDT compounds ( $o, p$ '- and $p, p^{\prime}$-DDT, -DDE, -DDD + p, $p$ '-DDMU, -DDNU), 84 PCB (polychlorinated biphenyl) congeners, and 38 long-chain alkylbenzenes (LCABs). The same analytical methods were used as those employed during the analysis of a core collected in 1992 as part of SCBNRDAP in support of the Montrose case. This provided a means of estimating $p, p$ '-DDE transformation rates for the period 1992-2003 through comparison of $p, p^{\prime}$-DDE whole-core inventories and $p, p$ '-DDE concentrations in coeval sediment layers.

The analyses show that the whole-core inventory of $p, p$ '-DDE $\left(\mu \mathrm{g} / \mathrm{cm}^{2}\right)$ decreased by 43.2 percent from 1992 to 2003, whereas inventories of $p, p^{\prime}$-DDMU and $p, p^{\prime}$-DDNU increased by 33.5 percent and 32.9 percent, respectively, during the same period. The estimated first-order $p, p$ '-DDE transformation rate based on whole-core inventories of $p, p$-DDE for the 1992 and 2003 cores was $0.051 \mathrm{yr}^{-1}$; mean and median rates for all sections of the cores were 0.052 and $0.045 \mathrm{yr}^{-1}$, respectively. These can be compared with estimates based on regression analysis of LACSD core data for the period 1991-2005, which yielded rates at LACSD station 3C of $0.089 \mathrm{yr}^{-1}$ and $0.047 \mathrm{yr}^{-1}$ for sediments above and below a sub-bottom depth of $10 \mathrm{~g} / \mathrm{cm}^{2}$, respectively. Rates based on direct comparison of wholecore inventories of $p, p$ '-DDE in cores collected by the LACSD at station 3C in 1991 and 2005 ranged from 0.047 to $0.085 \mathrm{yr}^{-1}$. The most comparable microcosm data for station 3C (Quensen and others, 2001; Deming and Carpenter, 2000) yielded rates from 0.073 to $0.151 \mathrm{yr}^{-1}$ and $0.23 \mathrm{yr}^{-1}$. Thus, the majority of the field data indicates a $p, p$ '-DDE transformation rate at station $3 \mathrm{C}$ of approximately 0.05 $\mathrm{yr}^{-1}$, lower than those obtained in the microcosm studies. Limited data are also available for other locations on the shelf. Based on comparison of whole-core inventories of $p, p$ '-DDE in sediments collected by the LACSD at station 6C in 1991 and 2005, rates from 0.013 to $0.028 \mathrm{yr}^{-1}$ were obtained These are approximately two to three times lower than those at station 3C. Estimates derived from regression analysis of LACSD data for the period 1991-2005 yielded rates at station 6C of 0.082 $\mathrm{yr}^{-1}$ and $0.019 \mathrm{yr}^{-1}$ for sediments above and below a depth of $10 \mathrm{~g} / \mathrm{cm}^{2}$, respectively.

The basis for direct comparison of $p, p$ '-DDE data in cores collected in 1992 and 2003 is the assumption that differences in concentration between the cores reflect losses of $p, p$ '-DDE over this time period. Support for this assumption comes from the fact that PCBs in these sediments show no evidence of reductive dechlorination, and PCB inventories in the two cores are the same within analytical error. In other words, the PCBs are conservative tracers of waste-derived contamination at this site. One of the two types of LCABs measured in this study, the tetrapropylene-based alkylbenzenes (TABs), are also believed to be persistent in anoxic sediments. They, too, behaved conservatively. This provides independent corroboration of the validity of direct core-to-core comparison of $p, p$ '-DDE concentrations for purposes of estimating dechlorination rates. By contrast, inventories of the other type of LCAB, the linear alkylbenzenes (LABs), exhibited a significant decline between 1992 and 2003 (-53.8 percent). This shows that, contrary to current views, the LABs are not recalcitrant in anoxic sediments.

From the field studies described above, estimates have been developed for first-order $p, p$ '-DDE transformation rates at several locations. The estimates derived from field data appear to be consistent with each other, raising confidence in their accuracy. It appears that the rate of dechlorination is higher away from the outfalls (for example, station 3C) than it is near them (for example, station 6C). This finding is in accord with results obtained in the microcosm studies and time series of inventories based on LACSD monitoring data, but the reasons for this pattern are unknown. Possibilities include differences in the: 1) microbial communities, 2) degree of inhibition of dechlorination activity, 3) bioavailability of $p, p$ '-DDE, and 4) redox conditions. Concerning the vertical variation in dechlorination rates within the sediment column, examination of the results of microcosm experiments 
and field data reveals inconsistencies that cannot be resolved without further data. For example, data from the direct comparison of 1992 and 2003 USGS cores collected near station 3C show that highest transformation rates occur at depths corresponding to the lower shoulder of the subsurface contaminant peak (38 to $44 \mathrm{~g} / \mathrm{cm}^{2}$ ). The only microcosm study carried out with a sediment core from station $3 \mathrm{C}$ gave high dechlorination rates at sub-bottom depths of $30-40 \mathrm{~cm}$, but no tests were performed on the overlying sediment intervals. Other microcosm experiments conducted with sediments from station $6 \mathrm{C}$ gave, in one case, higher dechlorination rates in the upper portion of the sediment column and, in another case, high rates in upper and lower portions of the core with low rates in between. These differences are due, at least in part, to variations in experimental design, most notably with respect to the availability of sulfate. More information would be needed to fully elucidate the factors controlling dechlorination rates on the shelf.

Using measured inventories of $p, p^{\prime}$-DDE, $p, p^{\prime}$-DDMU, and $p, p^{\prime}$-DDNU in the 1992 and 2003 cores and the average first-order $p, p^{\prime}$-DDE transformation rate obtained by direct comparison of these cores, a model for the long-term fate of the DDT compounds was developed. The model scenario presented in this report depends on a number of assumptions, several of which are not yet substantiated. According to model projections, the inventory of $p, p$ '-DDE near station $3 \mathrm{C}$ will continue to decline, whereas inventories of $p, p^{\prime}$-DDMU and $p, p^{\prime}$-DDNU reach maxima around 2002 and 2014, respectively, and then decline thereafter. These projections may prove useful as monitoring of the site proceeds, but gaps in knowledge of what controls the rate of $p, p$ '-DDE dechlorination will limit the ability to predict, let alone enhance, natural attenuation of this compound and to determine its ultimate fate. Additional monitoring near station $3 \mathrm{C}$ and at other locations would help improve understanding of the temporal and spatial variation in dechlorination rates on the Palos Verdes Shelf.

\section{Introduction}

The Sanitation Districts of Los Angeles County (LACSD) operate one of the largest wastewater treatment plants in the world. Located in Carson, CA, and encompassing $1.42 \mathrm{~km}^{2}$, the Joint Water Pollution Control Plant (JWPCP), discharges $1.325 \times 10^{9}$ liters of treated effluent each day to waters off Palos Verdes Peninsula (fig. 1A). The effluent is released through a system of submarine outfalls terminating at water depths of approximately 60 meters near White Point (fig. 1B). Upon entering the ocean, the effluent mixes rapidly with seawater and forms a buoyant plume that rises in the water column (Farley, 1990). During much of the year the water column off Palos Verdes is thermally stratified. Under these conditions, the plume is maintained below the pycnocline and is transported away from the outfall system by prevailing currents (Jones and others, 2002). Suspended particulate matter in the effluent coagulates upon contact with seawater, enhancing sedimentation of particle-bound contaminants (Hendricks and Eganhouse, 1993).

In the late 1960s and early 1970s it became apparent that the discharge of wastes from the JWPCP had resulted in contamination of sediments deposited on the Palos Verdes Shelf (Klein and Goldberg, 1970; McDermott and others, 1974; MacGregor, 1976). Although the contaminants were found to consist of a wide range of different substances (Stull and others, 1996; Eganhouse and Kaplan, 1988), the chemicals of greatest concern were determined to be the pesticide DDT and its degradation products, and the polychlorinated biphenyls (PCBs). These compounds are persistent, toxic, and they accumulate in tissues of living organisms. The uptake of DDT and PCBs from Palos Verdes Shelf sediments by indigenous marine life has been documented ever since the early 1970s (for example, Young and others, 1988), but it was the dramatic reproductive failure of the brown pelican during the 1960s off southern California that spurred concerns over the broader effects of DDT on the marine ecosystem (Anderson and others, 1975). 
The principal source of DDT to the JWPCP was the Montrose Chemical Corporation, Torrance, CA, which received a permit to discharge its manufacturing wastes into the sewer starting in 1953 . The discovery of high DDT concentrations in the JWPCP's influent and effluent in late 1969 and its sourcing to Montrose in March of 1970 have been described elsewhere (MacGregor, 1974; Chartrand, 1986). As much as 1500-2000 metric tons of DDT are believed to have been released by Montrose from 1953 to 1970, after which inputs to the sewer system were largely terminated. [Note: During the period 1947-1970, Montrose is estimated to have produced $0.48 \times 10^{6}$ metric tons of technical DDT, approximately $1 / 4$ of the total global production, making it the world's largest producer. Montrose ceased operation in 1982.] Approximately half of the material entering the JWPCP was discharged through the LACSD outfall system to waters of the Palos Verdes Shelf. As of 1992, 109 metric tons of total DDT (comprised of the $o, p$ '- and $p, p^{\prime}$ - isomers of DDT, DDD, and DDE + $p, p^{\prime}$-DDMU) were found in sediments of the shelf and upper slope (Lee, 1994a). This is roughly 10 to 15 percent of the amount of DDT that was discharged from the outfalls. The rest is presumed to have been transported to other parts of the Southern California Bight (Stull and others, 1996: Niedoroda and others, 1996).

The pattern of contamination in sediments of the Palos Verdes Shelf (as defined by $p, p^{\prime}$-DDE concentration, mass of total DDT per unit area, and acoustic profiles) can be described as an elongated ellipse parallel to the shoreline but extending predominantly to the northwest of the outfalls with highest mass accumulations centered along the 60-meter isobath (fig. 1B; Stull and others, 1996; Lee and others, 2002; Hampton and others, 2002, among others). DDT and PCB mass accumulations show steep gradients to the southeast of the outfall system and across the shelf. These patterns reflect the transport of effluent particles and sediments under the influence of subsurface currents on the shelf and upper slope that move mainly alongshelf toward the northwest (Noble and others, 2002).

In June 1990, the National Oceanic and Atmospheric Administration (NOAA) filed suit against Montrose Chemical Corporation (and a list of other defendants) under the natural resource damages provision of CERCLA (Comprehensive Environmental Response, Compensation, and Liability Act, also referred to as Superfund). In August 1997, while the case was still being litigated, the Palos Verdes Shelf was included by the U.S. Environmental Protection Agency (USEPA) on the National Priorities List as part of the Montrose Chemical Corporation Site listing. The lawsuit went to trial in October 2000, and a settlement was reached that December. In anticipation of the need for remedial action, the USEPA had already commissioned a pilot capping experiment that was undertaken by the U.S. Army Corps of Engineers (USACE) in August 2000. The purpose of the experiment was to investigate the feasibility of capping the contaminated sediments (U.S. Army Corps of Engineers, 2002). Subsequent investigations (U.S. Army Corps of Engineers, 2002; Science Applications International Corporation, 2005) have sought to determine whether the pilot capping was successful and if in situ capping remains a viable option for all or portions of the shelf.

Successful remediation of contaminated marine sediments requires information on the distribution, mobility, and biogeochemical fate of the contaminants of concern (National Academy of Sciences, 2001). The work described in this report is an effort to investigate the behavior and fate of the principal organic contaminants on the Palos Verdes Shelf, DDT and the PCBs. In particular, this report focuses on one aspect of the fate of these compounds, their biogeochemical transformation to other compounds and environmental factors that influence these rates. The results of this study will be incorporated into a larger investigation aimed at characterizing the status of the deposit and predicting its long-term fate (C.R. Sherwood and B. Ferré, written commun., 2007). Collectively, this information will be used by the USEPA to help develop a plan for remediation of the site. 


\section{Impetus for this study}

In late 2005 the LACSD completed chemical analyses of cores that had been collected on the Palos Verdes Shelf during the previous summer. For the first time, the list of targeted analytes included $p, p$ '-DDMU, a putative reductive dechlorination product of $p, p$ '-DDE. The LACSD found higher amounts of $p, p^{\prime}$-DDMU (relative to $p, p^{\prime}$-DDE) in these cores than had been reported by the USGS for cores collected in 1992 at nearby stations (Bailey and Costa, 1994; Eganhouse and others, 2000; fig. 23). NNote: The red bars in Figure 3 indicate high contamination zones within which decreases in percent $p, p$ '-DDMU are observed. The significance of this feature is described in a later section

(Environmental factors controlling DDE transformation rates).] This signaled the possibility that rates of reductive dechlorination of $p, p^{\prime}$-DDE on the Palos Verdes Shelf had changed at some point after the early 1990s. To verify these findings, the LACSD decided to reanalyze a pair of cores originally collected and analyzed in 1991, this time including $p, p^{\prime}$-DDMU among the targeted analytes. Although the primary purpose of the reanalysis was to verify that the results obtained for the 2005 cores were valid (by comparison of the 1991 core reanalysis results with 1992 USGS core data), an additional benefit of the exercise was the development of data for cores spanning 14 years using consistent analytical methodology. With respect to the relative abundance of $p, p$-DDMU, the 1991 core reanalysis showed very good agreement with results obtained by the USGS in 1992 (see discussion to follow), indicating that the measurements made by the LACSD on the 2005 cores were valid.

To further investigate the significance of these new findings and to develop information concerning the spatial variability of $p, p^{\prime}$-DDE $\rightarrow p, p^{\prime}$-DDMU transformation rates on the Palos Verdes Shelf, the U.S. Geological Survey (USGS) undertook a study in cooperation with the USEPA to: 1) analyze existing field and laboratory data, and 2) produce additional geochemical data for a core collected in 2003 by the USGS. The overall goal of this work was to provide a better understanding of the range of reductive dechlorination rates on the Palos Verdes Shelf and the environmental factors that control them. The project is part of an effort to model the long-term fate of the contaminated deposit under the so-called 'no action' scenario (C.R. Sherwood and B. Ferré, written commun., 2007). In this context, in situ DDE $\rightarrow$ DDMU transformation may be viewed as one of several processes contributing to the natural attenuation of the principal DDT compound in these sediments, $p$, $p$-DDE (Sherwood and others, 2002).

\section{Objectives}

The primary objectives of this study were to: 1) determine rates of reductive dechlorination of $p, p$ '-DDE in sediments near station 3C for the period 1992-2003, 2) determine if PCBs in these sediments had undergone reductive dechlorination, 3 ) assess the spatial variability of reductive dechlorination of $p, p^{\prime}$-DDE on the Palos Verdes Shelf, and 4) synthesize existing field and laboratory information on reductive dechlorination of $p, p^{\prime}$-DDE. In addition to the analyses for the DDT compounds and the PCBs, concentrations of two classes of synthetic surfactant chemicals, the longchain alkylbenzenes (LCABs) were determined. These measurements were made to provide ancillary information on sediment core chronology (molecular stratigraphy) and the validity of critical assumptions underlying calculation of in situ p,p'-DDE dechlorination rates (see discussion to follow).

\section{Background}

In the following sections background information is presented on the degradation of DDT, relevant studies that have been conducted on the Palos Verdes Shelf, and laboratory experiments focusing on the microbial reductive dechlorination of $p, p^{\prime}$-DDE. 


\section{DDT degradation}

In September 1939 a Swiss chemist, Dr. Paul Müller, discovered the insecticidal properties of DDT for which he received the Nobel Prize in Physiology or Medicine in 1948. Persistence was among the criteria Müller established for the ideal insecticide at the outset of his investigation. Ironically, it was this characteristic, combined with unanticipated adverse ecological effects and the development of insect resistance that ultimately led to the 1972 banning of DDT for most uses in the United States. Because of the widespread application of large amounts of technical DDT and its propensity for longrange transport, it soon became distributed globally leading to contamination of soils, sediments, and biota. During the 1960s and 1970s a tremendous amount of research was directed at understanding the pathways of DDT degradation (biotic and abiotic) and the fate of this pesticide in the environment, but this work largely ceased during the early 1980s as interest in DDT waned. Consequently, while analytical and microbiological (particularly anaerobic) techniques continued to advance during the ensuing 25 years, few systematic studies of the behavior of DDT and its metabolites were undertaken.

DDT can be transformed abiotically and through microbially-mediated reactions. Abiotic transformations include reductive dechlorination to form DDD in the presence of transition metals and reduced metal complexes (for example, porphyrins; Esaac and Matsumura, 1980) and photochemical or hydrolytic dehydrochlorination to form DDE (Zepp and others, 1977; Wolfe and others, 1977). The present understanding of the microbial degradation of DDT is primarily based on studies of pure cultures or microcosms. Reviews of the field (for example, Lal and Saxena, 1982; Rochkind-Dubinsky and others, 1987; Aislabie and others, 1997) have attempted to synthesize existing experimental and field data, but the pathways of degradation are still not fully understood. In particular, there is a paucity of information on the degradation of $p, p$ '-DDE (Aislabie and others, 1997), especially in marine systems. Until the experiments of Quensen and others (1998), DDE was considered a recalcitrant compound (for example, Callahan and others, 1979; Rochkind-Dubinsky and others, 1987; Stull and others, 1996, among others). This may be attributable to the fact that in many earlier studies, little attention was paid to the formation of relevant transformation products (DDMU, DDMS, DDNS) and the focus was clearly on degradation of DDT, not DDE.

A generalized scheme of potential pathways for microbial degradation of DDT is presented in Figure 4. It summarizes findings from a combination of specific studies involving pure cultures as discussed in the aforementioned reviews. The reactions depicted in this figure have not been verified for all systems and probably represent an oversimplification of the range of possible reactions that can occur in individual species and/or environments. Nevertheless, it is useful to discuss these reactions and place them in context. The predominant DDT metabolite in Palos Verdes Shelf sediments is $p, p$ '-DDE. This compound is believed to have been formed through hydrolytic and/or microbial dehydrochlorination shortly after discharge from the JWPCP outfall system (List, 1997; Eganhouse and others, 2000). This accounts for the very low abundance of $p, p$ '-DDT that was found in shelf sediments as early as 1972 (McDermott and others, 1974), only one year after inputs to the sewer system by Montrose Chemical Corporation were terminated. Under the reducing conditions that are likely to prevail in all but the upper few centimeters of the sediments on the Palos Verdes Shelf (Van Cappellen and Santschi, 1999), $p, p^{\prime}$-DDE could be transformed by hydrogenation (to form $p, p^{\prime}$-DDD) or by reductive dechlorination (to form $p, p^{\prime}$-DDMU). As discussed by Quensen and Tiedje (1997), evidence for the hydrogenation reaction in the literature is scant. Moreover, $p, p$ '-DDMU is present at concentrations that were 3 to 6 times those of $p, p^{\prime}$-DDD in 1992 (Eganhouse and others, 2000) and the relative abundance of $p, p$ '-DDD has changed little over time. At the same time, the relative abundance of $p, p^{\prime}$-DDMU has increased since 1992 and $p, p^{\prime}$-DDE abundance has decreased (see discussion to follow). This suggests that dechlorination (rather than hydrogenation) is the preferred transformation 
process under reducing conditions at the site. As shown in Figure 4, it is conceivable that a series of reductive dechlorination reactions leading from $p, p^{\prime}$-DDD and $p, p^{\prime}$-DDE should result in production of the following metabolites ( $\boldsymbol{p}, \boldsymbol{p}$ '-DDD: $p, p^{\prime}$-DDMS, $p, p^{\prime}$-DDNS; $\boldsymbol{p}, \boldsymbol{p}$ '-DDE: $p, p^{\prime}$-DDMU, $p, p^{\prime}$-DDNU). All of these compounds have now been identified in sediments of the Palos Verdes Shelf. Transformation of $p, p^{\prime}$-DDD to $p, p^{\prime}$-DDMU or $p, p^{\prime}$-DDMS to $p, p^{\prime}$-DDNU via dehydrochlorination is theoretically possible. However, this reaction is believed only to occur under oxidizing conditions (Lal and Saxena, 1982). Subsequent steps indicated in the scheme of Figure 2 (bottom row) involve oxidation, decarboxylation, and dehydrogenation reactions that may also require oxidizing conditions (Rochind-Dubinsky and others, 1987). With the exception of $p, p^{\prime}$-DBP, these products have not been observed as free constituents of the Palos Verdes Shelf sediments (Eganhouse and others, 2000). There is some evidence for the existence of 'bound' DDT metabolites that were released in the form of $p, p$ 'DDA and $p, p$ '-DDM by alkaline hydrolysis (Schwarzbauer, 2002).

\section{Previous work on the Palos Verdes Shelf}

The Palos Verdes Shelf may be the most intensively studied portion of the continental shelf in the world today. Indications of the potential for adverse ecosystem effects of wastewater-derived contaminants, such as DDT, led to formation of the Southern California Coastal Water Research Project (SCCWRP) in 1969. SCCWRP's mission is to understand the effects of human activities on the coastal ecosystem off southern California. To that end, SCCWRP has conducted investigations of the input, transport, fate, and effects of anthropogenic contaminants in the Southern California Bight, results of which are documented in a series of annual (and sometimes biennial) reports, technical memoranda, and journal articles (http://www.sccwrp.org). In addition, since 1981 the LACSD has maintained an ocean monitoring program on the Palos Verdes Shelf that includes assessments of physical oceanographic conditions, levels of contamination in sediment and biota, ecosystem health, and outfall condition. The program has evolved over time in response to regulatory agency requirements and knowledge gained; results of the annual surveys can be found in a series of reports (for example, LACSD, 2005). In 1987 the LACSD convened a meeting of experts to consider the current state of knowledge on processes affecting the fate of contaminated sediments on the Palos Verdes Shelf (Kolpack, 1987). The recommendations of this workshop were largely implemented during studies undertaken in the early 1990s by scientists from the USGS and other scientific organizations (U.S. Department of Justice, 1994a; Lee and Wiberg, 2002) as part of the SCBNRDAP in support of the case of United States of America, et al., vs. Montrose Chemical Corporation of California, et al., hereafter referred to as the "Montrose case.". Work related to a pilot capping study (U.S. Army Corps of Engineers, 2002) and its subsequent evaluation (Science Applications International Corporation, 2005) as well as the efforts in support of the USEPA's restoration efforts (C.R. Sherwood and B. Ferré written commun., 2007; M. A. Noble and others, written commun., 2007) have made substantial contributions to understanding of the site.

Of the above-mentioned field studies, only a few have provided valuable insights into the biogeochemistry of DDT on the Palos Verdes Shelf. In July and August 1971 MacGregor (1976) conducted a survey of DDT compounds in sediments from the Palos Verdes margin including Santa Monica Bay and San Pedro Basin, an area of $3,129 \mathrm{~km}^{2}$ (fig. 1A). The principal conclusion of the study was that parent DDT was transformed in shallow waters of the shelf (to DDE and DDD), but that in deeper waters, less transformation was apparent. On the shelf, DDE was approximately 10 times as abundant as parent DDT, whereas DDT was more abundant than DDE in waters north of Santa Catalina Island. Subsequent work indicates that the high parent DDT abundances observed by MacGregor (1976) in deeper waters of San Pedro Basin were most likely due to residues of Montrose acid wastes 
that were disposed of by barge in this area from 1947 to 1961 (Chartrand and others, 1985; Venkatesan and others, 1996; Zeng and Venkatesan, 1999). Owing to limitations of the analytical methods used at that time, it was not possible to separate $p, p$ '-DDMU from $o, p$ '-DDE, and the concentrations of the peak eluting in this region of the chromatogram was presented as a single analyte, ' $o, p$ '-DDE'. Analysis of three sediment samples collected on the Palos Verdes Shelf roughly 11 months after the initial survey showed that the inventory of ' $o, p$ '-DDE' (possibly including $p, p$ '-DDMU) had increased while that of $p, p$-DDE either was unchanged or had decreased. Notwithstanding the uncertainties of such measurements, MacGregor (1976) concluded that these changes could have been caused by 'metabolism,' additions of sediments depleted in parent DDT plus 'metabolism,' or loss of the surficial sediments (without 'metabolism'). From the present understanding of the site, MacGregor's data seem incomprehensible. They suggest that over the span of less than one year, a major proportion of the $p, p$ 'DDE was converted to $p, p$ '-DDMU, whereas there is little evidence that the composition of the DDTs changed between the early 1970s and the early 1990s, a span of 20 years, with $p, p$ '-DDE about 3 to 4 times more abundant than $p, p^{\prime}$-DDMU in 1992 (Eganhouse and others, 2000).

McDermott and others (1974) presented data for the $o, p^{\prime}$ - and $p, p^{\prime}$ - isomers of DDT, DDD and DDE in a gravity core collected at LACSD station 3C (Figure 5B) on the Palos Verdes Shelf in June 1972. Their data show compositions dominated at all depths by $p, p$ '-DDE (56.2-89.4 percent of total DDT; mean $=77.2$ percent) with no obvious trend with depth in the sediment column. The compositions were very similar to those determined by the LACSD at station 3C in 1981 (nine years later) and by Eganhouse and others (2000) near station 3C in 1992 (20 years later), suggesting that any changes in DDT composition that may have occurred at this site took place very rapidly and resulted in dominance by $p, p$ '-DDE. Unfortunately, it is not possible to know what the relative abundance of $p, p$ 'DDMU was in this core because this compound was not measured by McDermott and others (1974). However, it is clear that their data do not show the high abundances for the $o, p$ '-DDE peak reported by MacGregor (1976). The reason(s) behind the unusual results obtained by MacGregor (1976) for $o$, $p$ 'DDE (thought by MacGregor to include $p, p$ '-DDMU) in sediment samples collected in 1972 remains a mystery.

Based on a comparison of data for the Montrose effluent, sewer residues downstream from the Montrose discharge point, LACSD final effluent, and sediment cores collected in 1972, 1981, and 1992, Eganhouse and others (2000) concluded that effluent discharged to the Palos Verdes Shelf was probably dominated by parent DDT but that during and shortly after sedimentation, the majority of it was converted to DDE. Evidence for reductive dechlorination of $p, p$ '-DDE was noted by the presence of $p, p$-DDMU throughout the 1992 core and the fact that the abundance of this compound increased gradually with depth. However, based on its relatively low abundance (12 to 25 percent of total DDT) and the long period over which these sediments had been deposited (20 to 40 years) a low maximum rate of dechlorination, significantly slower than those obtained from microcosm experiments (Quensen and others (1998), was indicated (see discussion below). An attempt was made in this study to estimate in situ dechlorination rates based on age-dating of the sediments using molecular stratigraphy (Eganhouse and Pontolillo, 2000). However, these calculations depended upon the assumption that $p, p$-DDMU was a dead-end product, an assumption that was supported at that time by microcosm experiments (Quensen and others, 1998).

An important conclusion of the study by Eganhouse and others (2000) was that reductive dechlorination alone could not explain the observed decline in $p, p$ '-DDE inventories at station $3 \mathrm{C}$ during the 1980s and early 1990s (see Lee and others, 2002; Stull and others, 1996). Instead, most of the loss was attributed to upward mixing of contaminated sediments and release to overlying waters via desorption from sediments mobilized during resuspension events. This hypothesis was explored by 
Zeng and Venkatesan (1999) through an examination of core data and water-column measurements of DDT at various locations along the Palos Verdes Shelf and in Santa Monica Bay. A modeling exercise by Niedoroda and others (1996) explicitly ignored reductive dechlorination, but Sherwood and others (2002) attempted to assess the contribution of this process to declining $p$, $p$ '-DDE inventories near station $3 \mathrm{C}$. They concluded that in situ reductive dechlorination was potentially important but that large uncertainties in the spatial and temporal variation in $p, p$ '-DDE transformation rates prevented an accurate quantitative assessment.

\section{Laboratory studies}

The earliest known publication indicating the potential for reductive dechlorination of $p$, $p$-DDE to $p, p$ '-DDMU is that of Massé and others (1989). However, this study involved incubation of $p, p$ 'DDE in the presence of a pure culture of a Gram-negative bacterium (strain B-206). In 1998 Quensen and others published a paper on microcosm experiments they had performed using sediments from the Palos Verdes Shelf. The experiments involved slurries made from sediment cores collected at three stations (3C, 5C, and 8C; fig. 5B) that had been spiked with ${ }^{14} \mathrm{C}$-labelled DDE or ${ }^{14} \mathrm{C}$-labelled DDD (at approximately $100 \mu \mathrm{g} / \mathrm{dry} \mathrm{g}$ ) and mixed thoroughly. The purpose of this work was to determine if the $p, p$ '-DDMU found in Palos Verdes Shelf sediments was produced from $p, p^{\prime}$-DDE or from $p, p^{\prime}$-DDD. The microcosms were maintained at room temperature $\left(22\right.$ to $\left.25^{\circ} \mathrm{C}\right)$ under either sulfidogenic or methanogenic conditions and were sampled at 8-week intervals over a period of 32 weeks. Each sample was extracted with organic solvents and a portion of the extract was separated by thin-layer chromatography (TLC). Three bands identified by autoradiography as corresponding to $p, p$ '-DDE, $p, p^{\prime}-$ $\mathrm{DDD}$, and $p, p$-DDMU were scraped from the plates, and the ${ }^{14} \mathrm{C}$ activity of material in these bands was measured by liquid scintillation counting.

The results showed that there was little transformation of $p, p$ '-DDD and that only trace amounts of $p, p$ '-DDMU were produced from this precursor. In the case of $p, p$ '-DDE, transformation to $p, p^{\prime}-$ DDMU was found to occur after a lag period; no $p, p$ '-DDMU was produced in autoclaved controls. This proved conclusively that microorganisms in sediments from the Palos Verdes Shelf were capable of reductively dechlorinating $p, p$ '-DDE. Transformation rates for the methanogenic treatments were significantly greater than those for the sulfidogenic treatments (table 1), and measurable dechlorination started earlier in methanogenic microcosms. Dechlorination rates for sediments collected from different locations also differed, with highest rates overall under methanogenic conditions at station 3C. The rates, reported in units of $\mathrm{nmol} / \mathrm{g}$ dry sediment/day, were as follows: 1) sulfidogenic: 3C-0.17, 5C-0.22, $8 \mathrm{C}-0.10$, and 2) methanogenic: $3 \mathrm{C}-0.85,5 \mathrm{C}-0.36,8 \mathrm{C}-0.42$. Mass balances at the end of the ${ }^{14} \mathrm{C}-\mathrm{DDE}$ spiking experiments ranged from 85.5 to 93.2 percent with $p, p^{\prime}$-DDE and $p, p$ '-DDMU accounting for more than 99 percent of the recovered radioactivity in all treatments. Gas chromatography/mass spectrometry (GC/MS) was used to confirm the identity of $p, p^{\prime}$-DDMU in selected samples. Notwithstanding limitations of the separation and identification methods used for these experiments, there was no indication that reductive dechlorination of $p, p^{\prime}$-DDMU (to produce $p, p$ '-DDNU) had occurred.

In 2001, a second paper was published by Quensen and others (2001). This work was directed at addressing some of the limitations of the original study and to explore factors that might be responsible for controlling observed $p$, $p$ '-DDE dechlorination rates in Palos Verdes Shelf sediments. Microcosms were prepared in essentially the same manner as described in Quensen and others (1998), but the sediments came from stations 3C, 6C and 8C (fig. 5B), and varying amounts of sulfate and/or metabolizable organic matter were added to the microcosms as described below. Experiments were designed to investigate the effects of: 1) electron donor/sulfate concentration (station 3C), 2) availability 
of metabolizable organic matter (stations 3C, 6C, 8C), 3) depth in the sediment column (station 6C), and 4) temperature (station 3C). No GC or GC/MS confirmation of the identities of the DDT compounds in the TLC scrapings were reported. Sulfate depletion was defined as the point at which no more than one replicate (out of four) had greater than $0.52 \mathrm{mM}$ sulfate remaining, whereas the onset of methanogenesis was indicated when at least two replicates contained more than 0.5 percent methane in the headspace.

In the electron donor/sulfate concentration experiments, addition of carbon (pyruvate, lactate, glycerol) had the effect of reducing the lag time until sulfate depletion (onset of methanogenesis) occurred and DDE dechlorination began. The result was that by the end of these experiments (32 weeks), a greater extent of dechlorination was seen in microcosms amended with metabolizable organic matter. Without carbon addition, sulfate depletion and dechlorination occurred later, and dechlorination rates were inversely related to the amount of sulfate present at the beginning of the incubation. In general, as long as sulfate was present, the addition of carbon did not cause increased dechlorination rates.

In the experiments with unamended sediments (that is, no carbon added) from all three sites, $3 \mathrm{C}$ had the shortest lag time to sulfate depletion (and onset of methanogenesis), and 8C had the longest. The rate and extent of DDE dechlorination for unamended sediments followed the same pattern, with higher rates at 3C. These results mimicked those found in the previous study (Quensen and others, 1998). Addition of low amounts of metabolizable organic carbon $(2.1 \mathrm{mM})$ did not change this relationship, but addition of higher amounts $(8.4 \mathrm{mM})$ reduced the lag time to sulfate depletion and the onset of methanogenesis for all three sites, eliminating or diminishing the differences among them $(3 \mathrm{C}$ and $6 \mathrm{C}-8$ weeks, $8 \mathrm{C}-16$ weeks). The effect of carbon addition was to increase the dechlorination rate initially, but the extent of dechlorination was either not appreciably different from (3C) or less than (6C, $8 \mathrm{C}$ ) those observed in microcosms without carbon addition. From these observations, the authors concluded that factors other than the availability of metabolizable organic matter were responsible for the differences in dechlorination rate between stations. Suggested causes included differences in the: 1) microbial communities, 2) degree of inhibition of dechlorination activity, and 3 ) bioavailability of $p, p$ 'DDE.

In general, metabolizable organic matter appears to influence dechlorination activity indirectly through depletion of sulfate and the onset of methanogenesis, which favors higher rates of dechlorination. Quensen and others (2001) noted that sulfate depletion is mitigated in the marine environment by a ready supply of this anion in seawater. Consequently, the high rates found in methanogenic microcosms (which were sulfate-depleted) may not be relevant to effluent-affected sediments under actual field conditions. In fact, the authors noted that sulfate was found in all sediment samples collected from the Palos Verdes Shelf for these experiments. Minimum concentrations of 17.7 to $18.7 \mathrm{mM}$ occurred at depths of 45 to $50 \mathrm{~cm}$ below the sediment surface (Quensen and others, 2000). Van Cappellen and Santschi (1999) presented pore water chemistry profiles for cores collected from stations 6C and 8C in 1997. At station 6C they showed only a moderate decrease in sulfate concentration (from 30 to $28 \mathrm{mM}$ ), mostly occurring within the upper $5 \mathrm{~cm}$ of the sediment column, down to a depth of $20 \mathrm{~cm}$. Model calculations indicated that sulfate reduction was responsible for over 80 percent of the organic carbon oxidized within this depth range. Thus, it is presumed that sulfate reduction is the dominant terminal electron accepting process (TEAP) within Palos Verdes Shelf sediments. In the microcosm experiments, the concentration of sulfate did not appear to be as important in determining rates of dechlorination as whether sulfate was present. Dechlorination rate was constant as long as sulfate was present and increased only when it was depleted. Quensen and others (2001) hypothesized that the increased dechlorination rates in microcosms that became depleted in sulfate 
through addition of carbon may have been due to the use of DDE (instead of sulfate) as the terminal electron acceptor by abundant DDE-dechlorinating sulfate reducers once sulfate had become limiting.

Incubations of twelve 4-cm thick sections of a core from station $6 \mathrm{C}$ showed that the extent of DDE dechlorination was related to the degree of sulfate depletion (as indicted by methane formation). In general, methane formation and the extent of DDE dechlorination were higher in sections above 20 $\mathrm{cm}$ and below $40 \mathrm{~cm}$ in the core than in the intervening depths. In fact, from 24 to $40 \mathrm{~cm}$, residual amounts of sulfate were found in some sections and methane concentrations were undetectable in all sections until the end of the experiment (24 weeks; see Quensen and others, 2000, 2001). Because DDE dechlorination rates are slower under sulfidogenic than methanogenic conditions, it was reasoned that the low dechlorination rates in the 20 to $40 \mathrm{~cm}$ interval were due to incomplete depletion of sulfate. The cause of this was not explained, but Quensen and others (2001) hypothesized that it may be due to reduced rates of sulfate reduction and/or smaller numbers of dechlorinating microorganisms within the most highly contaminated layers of sediment (see discussion to follow).

Finally, microcosms with sediments from station $3 \mathrm{C}$ were incubated at three temperatures (10 ${ }^{\circ} \mathrm{C}, 15^{\circ} \mathrm{C}$, and $\sim 22{ }^{\circ} \mathrm{C}$ ) with and without addition of sulfate (table 1). Microcosms to which no sulfate had been added showed lag times to sulfate depletion (as indicated by detection of methane) and onset of DDE dechlorination (as indicated by appearance of DDMU) that were inversely related to temperature. In sulfidogenic microcosms (with sulfate addition sulfate was never completely depleted), DDE dechlorination rates were always lower than those under methanogenic conditions at a given temperature.

A third laboratory investigation was produced as evidence in the Montrose case (Deming and Carpenter, 2000). This microcosm study attempted to more closely approximate in situ conditions on the Palos Verdes Shelf including preservation of the original sediment texture, anaerobic conditions, in situ temperatures, and ambient DDE concentrations. Sediments were collected from stations 3C, 6C, and $8 \mathrm{C}$ in 1997. At each station a subcore was taken from the box core and cut into five 10-cm depth intervals. Each of these 10-cm long subcores were subcored again (with replication) using $13-\mathrm{cm}$ long tubes into which side injection ports had been tapped at $0.5 \mathrm{~cm}$ depth intervals. The $13-\mathrm{cm}$ long subcores were maintained anaerobically in the dark at $12{ }^{\circ} \mathrm{C}$ until they could be spiked with ${ }^{14} \mathrm{C}-\mathrm{DDE}$ ( 4.3 or $4.8 \mu \mathrm{g}$ into each port). No mixing of the spiked sediment was possible. [A separate experiment was conducted with some of the $6 \mathrm{C}$ subcores in which $32.5 \mu \mathrm{g}{ }^{14} \mathrm{C}$-DDE, approximately seven times as much material as was used in the other experiments, was added to each port. Also, one of the subcore replicates for each of the 10-cm sections was maintained frozen to serve as a time-zero control.] The subcores were then incubated for a period of 55 weeks after which they were sacrificed, and 2-cm sections were isolated and centrifuged. The solids were extracted with organic solvents, and the extracts were separated by TLC with detection using autoradiography. ${ }^{14} \mathrm{C}$ activity in the extracted pore water as well as bands on the thin-layer plates corresponding to DDE and DDMU were measured by liquid scintillation. Average recoveries of ${ }^{14} \mathrm{C}$ activity were as follows: $3 \mathrm{C}$ : 90 percent, $6 \mathrm{C}: 74$ percent, $8 \mathrm{C}$ : 77 percent. Data for five $2-\mathrm{cm}$ sections of the $3 \mathrm{C}$ core showed that combined ${ }^{14} \mathrm{C}$ activity of DDE and DDMU accounted for more than 98 percent of the recovered radioactivity. GC or GC/MS confirmation of the identities of the DDT compounds in the TLC scrapings was not reported. The only exception to this procedure was the unexplained absence of data for all but the 30 to $40 \mathrm{~cm}$ depth interval of the $3 \mathrm{C}$ core.

As in the case of the slurry microcosm studies of Quensen and others $(1998,2001)$, a spatial gradient in dechlorination rates was observed in these experiments, with highest rates reported for station $3 \mathrm{C}$ and lowest rates at station $8 \mathrm{C}$ (table 1). In microcosms with sediment from stations $6 \mathrm{C}$ and $8 \mathrm{C},{ }^{14} \mathrm{C}$-DDMU appeared in 80 percent of the core sections tested. Highest rates of dechlorination were 
found in the upper subsurface strata of these two cores $(6 \mathrm{C}: 2$ to $30 \mathrm{~cm}, 8 \mathrm{C}: 2$ to $22 \mathrm{~cm})$. This is in contrast to findings of Quensen and others (2001) who reported higher dechlorination rates above $20 \mathrm{~cm}$ and below $40 \mathrm{~cm}$ in the sediment column at station 6C. Data for only the 30 to $40 \mathrm{~cm}$ section of the $3 \mathrm{C}$ core were presented by Deming and Carpenter (2000). However, in contrast with the 6C and 8C cores, the $3 \mathrm{C}$ core showed very high rates within this depth range.

The elevated (7 times) spiking experiment conducted with the 6C core (Deming and Carpenter, 2000) resulted in higher DDE dechlorination rates and an increase in the range of depths over which dechlorination was observed. The magnitude of the increase in dechlorination rate was not proportional to the amount of ${ }^{14} \mathrm{C}$-DDE added. Lower than proportional responses were found in the upper dechlorination zone ( 2 to $22 \mathrm{~cm}$ ), whereas greater than proportional responses (up to 30 times) were observed in the underlying sediment $(24$ to $36 \mathrm{~cm})$; at greater depths the response, although variable, was roughly proportional to the spiking concentration. Deming and Carpenter (2000) hypothesized that the lower than proportional response in the upper portion of the sediment column indicated that the bacterial colonies in this zone were approaching maximal rates of dechlorination. By contrast, they believed that dechlorinating organisms in the 24 to $36 \mathrm{~cm}$ depth interval were adapted to higher concentrations of DDE, but that the lower absolute rates of dechlorination at these depths reflected a limitation in the availability of metabolically required substrates and/or nutrients due to increased burial. Deming and Carpenter (2000) suggested that because the most rapid dechlorination was occurring in the upper part of the sediment column $(6 \mathrm{C}, 8 \mathrm{C})$ increased burial, as in capping, would serve to reduce dechlorinating activity and could "...bring remediation to a precipitous halt".

To summarize, the studies of Quensen and others $(1998,2001)$ and Deming and Carpenter (2000) showed similarities and differences. Some of the differences are almost certainly attributable to variations in experimental design. All of the experiments demonstrate that dechlorinating capacity exists at several locations on the Palos Verdes Shelf (for example, 3C, 5C, 6C, 8C; fig. 5B) and at these locations, activity can occur almost at all depths in the sediment column. In addition, it is clear that there is a consistent difference in dechlorination rate with respect to location, with sediments from station $3 \mathrm{C}$ exhibiting higher rates than those nearer the outfalls (for example, $6 \mathrm{C}, 8 \mathrm{C}$ ). This is consistent with the observations of the change in inventories of $p, p$ '-DDE at these locations from 1981 to 1997 (Lee and others, 2002). At station 6C different results were obtained by Deming and Carpenter (2000) and Quensen and others (2001) with regard to the vertical distribution of dechlorination activity and rates. Deming and Carpenter (2000) reported that highest dechlorination rates were in the upper layers of the sediment column (8C: 2 to $30 \mathrm{~cm}$ and 6C: 2 to $22 \mathrm{~cm})$, whereas Quensen and others (2000, 2001) found highest rates in the 0 to $20 \mathrm{~cm}$ interval and at depths greater than $40 \mathrm{~cm}$ in the $6 \mathrm{C}$ core. Although data for the entire length of the core taken at station $3 \mathrm{C}$ were not reported by Deming and Carpenter (2000), they observed the highest rates for any station within the 30 to $40 \mathrm{~cm}$ depth interval tested. Without knowing the results for the 0 to $30 \mathrm{~cm}$ section of this core, one is left to speculate about the reasons behind the dramatic differences in dechlorination activity at stations $3 \mathrm{C}, 6 \mathrm{C}$ and $8 \mathrm{C}$ and its vertical variation in the Deming and Carpenter (2000) study. Consequently, the relationship between sediment depth and dechlorination activity remains unclear.

Table 1 provides estimates of the DDE dechlorination rates reported in or calculated from the aforementioned microcosm studies. The data are not perfectly comparable because of differences in experimental procedures and conditions. For example, the depth to which sediments were sampled for use in the slurry microcosms (Quensen and others, 1998, 2001) was not specified despite the fact that dechlorination rates were shown to vary with depth (Quensen and others, 2001). Moreover, most of the microcosm experiments described by Quensen and others $(1998,2001)$ were not maintained at or near the in situ temperature of the Palos Verdes sediments $\left(10\right.$ to $\left.12{ }^{\circ} \mathrm{C}\right)$, whereas those of Deming and 
Carpenter (2000) were. Other differences included the level of spiking, whether the spiked sediments were mixed or not, whether sulfate and methane were monitored, and the frequency of sampling.

Deming and Carpenter (2000) compared their dechlorination rates with those reported by Quensen and others (1998) by correcting for differences in incubation temperature, incubation time, and the amount of DDE spiked into the microcosms. They reported that the 'corrected' rates of Quensen and others (1998) were about 2 times greater than the highest rates obtained in their study (for stations $3 \mathrm{C}, 8 \mathrm{C}$ ). However, the authors did not indicate whether they were referring to rates obtained under methanogenic or sulfidogenic conditions. As shown in table 1, median first-order dechlorination rates reported by Deming and Carpenter (2000) for stations 3C and 8C were closer to those observed by Quensen and others (1998) under sulfidogenic conditions than those found under methanogenic conditions. The latter experiments were conducted at room temperature, and as illustrated by data for station 3C (table 1; Quensen and others 2001), there is a statistically significant relationship between dechlorination rate and temperature. For this reason, it is perhaps more relevant to compare Deming and Carpenter's results with data acquired by Quensen and others (2001) near the in situ temperature $\left(10\right.$ to $\left.12{ }^{\circ} \mathrm{C}\right)$. As shown in table 1, dechlorination rates reported by Deming and Carpenter (2000) in deeper portions of the core from station $3 \mathrm{C}$ fall between those obtained by Quensen and others (2001) under sulfidogenic and methanogenic conditions within the same approximate in situ temperature range $\left(10\right.$ to $\left.15^{\circ} \mathrm{C}\right)$. Because Deming and Carpenter (2000) did not measure sulfate or methane concentrations in their microcosms, it is unclear what TEAPs were dominant in their experiments. Consequently, it is difficult to place their rates in context with either the studies of Quensen or others $(1998,2001)$ or those obtained from field data. 
Table 1. Summary of DDE dechlorination rate data from laboratory microcosm experiments.

\begin{tabular}{|c|c|c|c|c|c|}
\hline Station & $\begin{array}{c}\text { Sample collection } \\
\text { date }\end{array}$ & $\begin{array}{l}\text { Depth interval tested } \\
\text { (cm) }\end{array}$ & Conditions $^{a}$ & $\begin{array}{c}\text { Dechlorination rate } \\
\left(y r^{-1}\right)^{b}\end{array}$ & $\begin{array}{l}\text { Half-life } \\
\text { (year) }^{c}\end{array}$ \\
\hline & & & Quensen and others (1998) & & \\
\hline \multirow[t]{2}{*}{$3 C$} & not reported & not reported & sulfidogenic, $22-25^{\circ} \mathrm{C}$ & 0.197 & 3.5 \\
\hline & & not reported & methanogenic, $22-25^{\circ} \mathrm{C}$ & 0.987 & 0.7 \\
\hline \multirow[t]{2}{*}{$5 \mathrm{C}$} & not reported & not reported & sulfidogenic, $22-25^{\circ} \mathrm{C}$ & 0.255 & 2.7 \\
\hline & & not reported & methanogenic, $22-25^{\circ} \mathrm{C}$ & 0.418 & 1.7 \\
\hline \multirow[t]{3}{*}{$8 \mathrm{C}$} & not reported & not reported & sulfidogenic, $22-25^{\circ} \mathrm{C}$ & 0.116 & 6.0 \\
\hline & & not reported & methanogenic, $22-25^{\circ} \mathrm{C}$ & 0.488 & 1.4 \\
\hline & & & Quensen and others (2001) & & \\
\hline $3 \mathrm{C}$ & not reported & not reported & sulfidogenic, $10^{\circ} \mathrm{C}$ & 0.073 & 9.5 \\
\hline $3 C$ & not reported & not reported & sulfidogenic, $15^{\circ} \mathrm{C}$ & 0.151 & 4.6 \\
\hline $3 C$ & not reported & not reported & sulfidogenic, $\sim 22{ }^{\circ} \mathrm{C}$ & 0.671 & 1.0 \\
\hline $3 \mathrm{C}$ & not reported & not reported & methanogenic, $10^{\circ} \mathrm{C}$ & 0.395 & 1.8 \\
\hline $3 \mathrm{C}$ & not reported & not reported & methanogenic, $15^{\circ} \mathrm{C}$ & 0.572 & 1.2 \\
\hline \multirow[t]{2}{*}{$3 C$} & not reported & not reported & methanogenic, $\sim 22^{\circ} \mathrm{C}$ & 1.024 & 0.7 \\
\hline & & & $\begin{array}{l}\text { Deming and Carpenter } \\
(2000)\end{array}$ & & \\
\hline $3 C$ & 9/97 & $30-40 \mathrm{~cm}$ & TEAP unspecified, $12^{\circ} \mathrm{C}$ & 0.231 & 3 \\
\hline $6 \mathrm{C}$ & 9/97 & $2-30 \mathrm{~cm}$ & TEAP unspecified, $12^{\circ} \mathrm{C}$ & 0.087 & 8 \\
\hline $8 \mathrm{C}$ & 9/97 & $2-22 \mathrm{~cm}$ & TEAP unspecified, $12^{\circ} \mathrm{C}$ & 0.069 & 10 \\
\hline
\end{tabular}

${ }^{a}$ Experimental conditions as described in publications. TEAP=terminal electron accepting process.

${ }^{b}$ Dechlorination rates for Quensen and others (1998) calculated as: rate $\left(\mathrm{yr}^{-1}\right)=\left\{\left[\mathrm{nmol}{ }^{14} \mathrm{C}-\mathrm{DDE} / \mathrm{g}\right.\right.$ sediment-day $] /\left[\mathrm{nmol}{ }^{14} \mathrm{C}-\right.$

DDE added/g sediment]\} x 365 days/yr. Rates for Deming and Carpenter (2000) calculated from reported median half-lives as: rate $\left(\mathrm{yr}^{-1}\right)=\ln (2) /$ half-life $(\mathrm{yr})$.

${ }^{c}$ DDE half-lives for Quensen and others $(1998,2001)$ calculated from first-order dechlorination rates, for Deming and Carpenter (2000) reported as median values for specified 'active zones' given in third column of this table.

\section{Methods}

Data to be discussed in this report come from the analysis of sediment cores collected as part of several different sampling programs. Sources of these data and their uses are described in the sections below, whereas table 2 summarizes information about the organizations that collected the cores, the dates of collection, the sampling devices used and the laboratories that conducted chemical analyses. The central focus of this report is a comparison of two box cores collected by the USGS from the same location in 1992 and 2003 (cores 124B1-DDT and 124B1-CH, respectively; table 2, fig. 5A). The 1992 core was collected as part of the Southern California Bight Natural Resource Damage Assessment Program (cruise F-5-92-SC; http://walrus.wr.usgs.gov/infobank/f/f592sc/html/f-5-92-sc.meta.html), which was conducted in association with the Montrose case (United States of America, et al., vs. Montrose Chemical Corporation of California, et al.). The 2003 core was collected as part of a subsequent USGS cruise unrelated to the Montrose case (cruise A-1-03-SC; http://walrus.wr.usgs.gov/infobank/a/a103sc/html/a-1-03-sc.meta.html). For these two cores, the methods of collection, preservation, processing and analysis were virtually identical in all respects. 
Most of these methods are described in reports published as part of the Southern California Damage Assessment List of Expert Witness Reports issued by the U.S. Department of Justice on October 4, 1994 (U.S. Department of Justice, 1994a). Pertinent aspects can also be found in a special volume of the journal, Continental Shelf Research, in 2002 (Lee and Wiberg, 2002) and elsewhere (Eganhouse and others, 2000; Eganhouse and Pontolillo, 2000; Wheatcroft and Martin, 1996). In the case of the analytical chemistry methods used for cores 124B1-DDT (1992) and 124B1-CH (2003), standard operating procedures (SOPs) were originally prepared in 1992-93 in compliance with the Southern California Damage Assessment Analytical Chemistry Quality Assurance Plan (Manen and others, 1994). These SOPs, updated in 2006 for purposes of the present study (Palos Verdes Remediation Project-PVRP), are appended to this report. A concise description of the analytical chemistry methods can also be found in two papers published in Marine Chemistry (Eganhouse and others, 2000; Eganhouse and Pontolillo, 2000). 
Table 2. Summary information for cores collected on the Palos Verdes Shelf that are discussed in this report.

\begin{tabular}{|c|c|c|c|c|c|}
\hline Station ${ }^{\mathrm{a}}$ & Core & Study ${ }^{b}$ & $\begin{array}{l}\text { Collection } \\
\text { device }^{\circ}\end{array}$ & Date collected & $\begin{array}{l}\text { Analytical } \\
\text { laboratory }\end{array}$ \\
\hline 522 & 124B1 & SCBNRDAP & B & $7 / 6 / 1992$ & USGS, ADL \\
\hline 522 & 124B1 & SCBNRDAP & B & $7 / 29 / 2003$ & USGS \\
\hline 550 & 169B1 & SCBNRDAP & B & $7 / 11 / 1992$ & ADL \\
\hline 550 & $177 \mathrm{G} 2$ & SCBNRDAP & $G$ & 7/13/1992 & $\mathrm{ADL}$ \\
\hline 557 & 127B1 & SCBNRDAP & $B$ & $7 / 7 / 1992$ & $\mathrm{ADL}$ \\
\hline 556 & $147 \mathrm{~B} 3$ & SCBNRDAP & B & $7 / 9 / 1992$ & $\mathrm{ADL}$ \\
\hline 556 & $179 \mathrm{G} 3$ & SCBNRDAP & G & $7 / 13 / 1992$ & $\mathrm{ADL}$ \\
\hline 564 & 171B1 & SCBNRDAP & B & $7 / 12 / 1992$ & ADL \\
\hline 564 & $181 \mathrm{G} 2$ & SCBNRDAP & G & $7 / 13 / 1992$ & $\mathrm{ADL}$ \\
\hline & & & & & \\
\hline $3 C$ & $3 \mathrm{C} 1$ & LACSD-OMP & G & $4 / 8 / 1981$ & LACSD \\
\hline $3 C$ & $3 \mathrm{C} 2$ & LACSD-OMP & G & $7 / 24 / 1991$ & LACSD \\
\hline $3 C$ & $3 \mathrm{C} 1$ & LACSD-OMP & $G$ & $6 / 8 / 2005$ & LACSD \\
\hline $3 C$ & $3 \mathrm{C} 2$ & LACSD-OMP & G & $6 / 8 / 2005$ & LACSD \\
\hline $3 C$ & $3 \mathrm{C} 3$ & LACSD-OMP & G & 6/8/2005 & LACSD \\
\hline $5 \mathrm{C}$ & $5 \mathrm{C} 1$ & LACSD-OMP & G & $6 / 8 / 2005$ & LACSD \\
\hline $5 \mathrm{C}$ & $5 \mathrm{C} 2$ & LACSD-OMP & G & $6 / 8 / 2005$ & LACSD \\
\hline $5 \mathrm{C}$ & $5 \mathrm{C} 3$ & LACSD-OMP & G & $6 / 8 / 2005$ & LACSD \\
\hline $6 \mathrm{C}$ & $6 \mathrm{C} 1$ & LACSD-OMP & G & $7 / 24 / 1991$ & LACSD \\
\hline $6 C$ & $6 \mathrm{C} 1$ & LACSD-OMP & G & $6 / 8 / 2005$ & LACSD \\
\hline $6 \mathrm{C}$ & $6 \mathrm{C} 2$ & LACSD-OMP & G & $6 / 8 / 2005$ & LACSD \\
\hline $6 \mathrm{C}$ & $6 \mathrm{C} 3$ & LACSD-OMP & $G$ & $6 / 8 / 2005$ & LACSD \\
\hline$A$ & $\mathrm{~A} / 7 \mathrm{CX}-1$ & SAIC-SDS & G & $7 / 17 / 2004$ & WHGEL \\
\hline A & A/7CX-2 & SAIC-SDS & G & $7 / 17 / 2004$ & WHGEL \\
\hline$A$ & $\mathrm{~A} / 7 \mathrm{CX}-3$ & SAIC-SDS & G & $7 / 17 / 2004$ & WHGEL \\
\hline A & A-1 & SAIC-SDS & $\mathrm{P}$ & $7 / 15 / 2004$ & WHGEL \\
\hline A & A-2 & SAIC-SDS & $\mathrm{P}$ & $7 / 15 / 2004$ & WHGEL \\
\hline A & A-3 & SAIC-SDS & $\mathrm{P}$ & $7 / 15 / 2004$ & WHGEL \\
\hline $7 \mathrm{C}$ & 7C-10089 & SAIC-SDS & $\mathrm{G}$ & $7 / 17 / 2004$ & WHGEL \\
\hline $7 \mathrm{CB}$ & 7CB-10085 & SAIC-SDS & G & $7 / 17 / 2004$ & WHGEL \\
\hline $7 \mathrm{C}$ & 7C-9150 & SAIC-SDS & $P$ & $7 / 14 / 2004$ & WHGEL \\
\hline $7 \mathrm{CB}$ & 7CB-9154 & SAIC-SDS & $P$ & $7 / 14 / 2004$ & WHGEL \\
\hline
\end{tabular}

${ }^{a}$ See Figures 5,6 for station locations.

${ }^{\mathrm{b}}$ SCBNRDAP $=$ Southern California Bight Natural Resource Damage Assessment Program, LACSD-OMP $=$ Los Angeles County Sanitation Districts Ocean Monitoring Program, SAIC-SDS $=$ Science Applications International Corporation Sediment Displacement Study.

${ }^{c} \mathrm{~B}=$ box corer, $\mathrm{G}=$ gravity corer, $\mathrm{P}=$ piston corer.

${ }^{\mathrm{d}} \mathrm{USGS}=\mathrm{USGS} /$ Reston,VA, ADL = Arthur D. Little, Inc./Cambridge, MA, LACSD = Los Angeles County Sanitation Districts/Whittier, CA, WHGEL = Woods Hole Group Environmental Laboratories/Raynham,

MA 


\section{Data sources}

Data presented in this report come from four sources. The first source is the extensive compilation of geochemical core data that was generated by the USGS for the Southern California Bight Natural Resource Damage Assessment Program from 1992 to 1994. These data can be found in reports by Lee (1994a) and Bailey and Costa (1994) with further description in Lee and others (2002). During a cruise that took place in the summer of 1992, the USGS collected box and gravity cores on a sampling grid across the Palos Verdes shelf and slope (fig. 5A). A subset of these cores was selected for chemical analysis; the selected cores were sectioned at discrete depth intervals and analyzed for seven DDT compounds ( $p, p^{\prime}-$ and $o, p^{\prime}-$ isomers of DDT, DDD, and DDE $+p, p^{\prime}$-DDMU), and 41 polychlorinated biphenyl (PCB) congeners by a contract laboratory (Arthur D. Little, Inc., Cambridge, MA). These data were then compiled and reported in the Southern California Damage Assessment List of Expert Witness Reports (U.S. Department of Justice, 1994a).

Some of the cores, intentionally collected near LACSD stations along the 60-meter isobath, capture the complete depositional history of the outfall system and were, therefore, analyzed in greater detail (for example, table 2). Chemical data for these cores are used in this report to depict the spatial variation of $p$, $p$-DDMU abundance on the Palos Verdes Shelf. One of these box cores, designated 124B1, was collected by the USGS in 1992 at station 522 (fig. 5A, table 2) near to, but inshore of, LACSD station 3C (compare fig. 5A-5B). A subcore from this box core, namely, 124B1-DDT was analyzed by both Arthur D. Little and USGS (Reston, VA) laboratories. The USGS analyte list included 43 additional PCB congeners and two classes of long-chain alkylbenzenes (LCABs) not measured by Arthur D. Little. The additional measurements were made in order to establish sediment chronology via molecular stratigraphy (Eganhouse and Pontolillo, 2000). In this report data for subcore 124B1-DDT (1992) are used to develop $p$, $p$ '-DDE transformation rate estimates by direct comparison with a subcore from the box core collected by the USGS at the same location in 2003 (124B1-CH).

The second source of data is the Los Angeles County Sanitation Districts (LACSD). As a voluntary part of their ocean monitoring program, the LACSD has collected gravity cores from a 44+ station sampling grid on the Palos Verdes Shelf on a biennial basis since 1981 (fig. 5B; Note: only 40 of the stations are shown.). The cores were frozen, sectioned, and analyzed for a list of contaminants including the $p, p$ '- and $o, p$ '- isomers of DDT, DDE and DDD (Lee, 1994b). Not all stations have been occupied in alternating years, but certain key stations along the 60-meter isobath (for example, 3C, 6C) have been sampled consistently, often with replicate cores being collected. This long-term data set is the basis for an analysis of sedimentation and p,p'-DDE loss rates (C.R. Sherwood and B. Ferré, written commun., 2007). For purposes of this report, three replicate cores collected at each of three LACSD stations (3C, 5C, and 6C) in 2005 and single cores (one of three replicates) collected at stations 3C and 6C in 1991 (table 2) are of particular interest because, for the first time, the measurements included $p, p$ '-DDMU. The 1991 and 2005 core data are used in this report to estimate $p, p$ '-DDE $\rightarrow p, p$ '-DDMU transformation rates (through comparison of whole-core inventories) and to plot spatial distributions of $p, p$ '-DDMU abundance for comparison with the USGS 1992 core data.

The third source of data is the sediment displacement study report (Science Applications International Corporation, 2005). These data are for a very restricted area near LACSD station $7 \mathrm{C}$ (compare fig. 5B-5C-5D) where intensive gravity and piston coring was undertaken in 2004 for the purpose of evaluating the magnitude and extent of sediment displacement subsequent to a pilot capping experiment conducted by U.S. Army Corps of Engineers in 2000-2001 (U.S. Army Corps of Engineers, 2002; table 2). Again, $p, p$ '-DDMU was included in the list of targeted analytes along with the $p, p^{\prime}$ - and $o, p^{\prime}$ - isomers of DDT, DDE and DDD. These data are used in this report for comparison 
of $p, p$ '-DDMU abundance in the capping cell sediments with the LACSD [1991, 2005] and USGS [1992] data sets.

The fourth source of data are chemical analyses carried out in 2006 by the USGS (Reston, VA) on a subcore from a box core collected by the USGS in 2003 near LACSD station 3C (namely, 124B1$\mathrm{CH}$; see fig. 5A-5B, table 2). The analytical results for cores 124B1-DDT (1992) and 124B1-CH (2003) provide a means of estimating $p, p^{\prime}$-DDE transformation rates near LACSD station $3 \mathrm{C}$ during the period from 1992 to 2003 through comparison of whole-core inventories and $p, p^{\prime}$-DDE concentrations at coeval depth intervals.

\section{Sampling procedures}

Sediment coring procedures used by the LACSD and SAIC can be found elsewhere (Lee, 1994b; Science Applications International Corporation, 2005) and will not be described further. In the case of the USGS sediment coring operations in 1992, detailed information on the navigation, box coring and subcoring operations are given in Hamer (1994) and Lee and Kayen (1994). These procedures changed only in minor details during the 2003 cruise. A brief description is provided here.

Sediment coring was conducted during cruises in 1992 and 2003. In 1992 navigation was primarily by differential GPS (geostationary positioning system); microwave transponder (Delnorte) and non-differential GPS were used as backup and for comparative purposes. In 2003 position data were collected with the USGS-designed YoNav navigation system with input from a CSI wireless DGPS Max differential GPS receiver (Gann, 1992). Sediment cores were collected in both years with a $0.06 \mathrm{~m}^{2}$ Naval Electronics Lab (NEL) box corer, which upon retrieval was subsampled using an electrically-driven cylindrical fixed piston subcoring device. Up to four subcores were obtained from a single box, pre-cleaned 8.3-cm o.d. polycarbonate liner and end caps being used for the subcore dedicated to organic geochemical analysis. The subcores were assigned alphanumeric designations in the following manner. Cores were identified by numeric prefixes (for example, 124), a letter designating the type of sampler (for example, ' $\mathrm{B}$ ' for box core, ' $\mathrm{G}$ ' for gravity core), and a number that indicated the number of corer deployments. Thus, a box core successfully collected from station 522 on the first attempt in 1992 was assigned a base name of 124B1 (see table 2). Subcores were differentiated by codes appended to their base names to indicate their intended purpose. For example, subcores taken in 1992 for organic geochemical analysis were given the suffix 'DDT' (hence, 124B1DDT). In 2003 procedures were identical to those described above except that the name of the subcore for organic geochemical analysis was appended with a suffix of ' $\mathrm{CH}$ ' (indicating 'chlorinated hydrocarbons'), rather than 'DDT' (hence, 124B1-CH). Immediately after collection, all subcores were tested with a whole-core logging system that yielded bulk density measurements at $1-\mathrm{cm}$ intervals (Kayen and others, 1999). Subcores for organic geochemical analysis were then placed upright in a freezer and maintained at $-20^{\circ} \mathrm{C}$ until they could be transported to the laboratory in a frozen state.

For purposes of estimating $p, p^{\prime}$-DDE transformation rates, subcores of the box cores collected from station 522 (fig. 5A) in 1992 and 2003 were analyzed by the USGS (Reston, VA) for chlorinated hydrocarbons (DDTs, PCBs), long-chain alkylbenzenes (LCABs) and other bulk properties. The 1992 core was collected on 6 July at $33^{\circ} 43^{\prime} 50.2^{\prime \prime} \mathrm{N}, 118^{\circ} 24^{\prime} 4.7^{\prime \prime} \mathrm{W}$, water depth $=57 \mathrm{~m}$. The 2003 box core was collected on 29 July at $33^{\circ} 43^{\prime} 49.6^{\prime} \mathrm{N}, 118^{\circ} 24^{\prime} 5.2^{\prime \prime} \mathrm{W}$, water depth $=57 \mathrm{~m}$ (fig. 6). The time between core collection is 4,040 days or 11.07 years, and the nominal distance between sampling positions is $22 \pm 5$ meters. 


\section{Analytical chemistry}

Information on the processing of sediment cores by Arthur D. Little (1992 USGS cores), the LACSD, and SAIC can be found elsewhere (Manen, 1994; U.S. Department of Justice, 1994b; U.S. Department of Justice, 1994c; Lee, 1994b; Science Applications International Corporation, 2005). Detailed information on the procedures used by the USGS (Reston, VA) for processing of the 1992 and 2003 124B1 sediment cores are provided in the SOPs appended to this document (see appendices 1-7). What follows are brief descriptions of these procedures.

\section{Materials}

In the laboratory only glass, stainless steel, aluminum, and Teflon ${ }^{\mathrm{TM}}$ implements were used in handling samples. Procedures for cleaning glassware and other implements can be found in appendix 1. In general, all utensils were washed in warm water and a non-alkylbenzene sulfonate-bearing detergent (Alcojet ${ }^{\mathrm{TM}}$ ), rinsed thoroughly with deionized water, and either combusted at $425^{\circ} \mathrm{C}$ for at least 4 hours (Pyrex ${ }^{\mathrm{TM}}$ glassware), sonicated in dichloromethane (DCM) and methanol (MeOH) followed by oven drying at $110{ }^{\circ} \mathrm{C}$ (stainless steel and borosilicate glass), or sonicated in solvents and air dried (Teflon ${ }^{\mathrm{TM}}$, aluminum).

Solvents (hexane, DCM, MeOH) of the highest quality obtainable (Burdick \& Jackson, glass distilled, Pesticide Residue Analysis grade) were tested for purity prior to use. Enough of each solvent was purchased at the beginning of the project to ensure that all samples could be extracted and processed with the same pre-tested lot. Milli- $\mathrm{Q}^{\mathrm{TM}}$ water used in the back extraction of $\mathrm{MeOH}$ extracts and for deactivation of the column chromatography adsorbents (see below) was pre-extracted with DCM and tested for purity prior to use. Adsorbents used for column chromatographic fractionation of sediment extracts (silica gel, alumina) were cleaned before activation. After deactivation, the adsorbents were tested to ensure proper activity and performance (appendix 2). Other reagents $(\mathrm{HCl}$, anhydrous $\mathrm{Na}_{2} \mathrm{SO}_{4}$, activated $\mathrm{Cu}^{\circ}$ ) were also purified prior to use.

Chemicals used for instrument calibration, quantitation, and recovery assessment were obtained either as certified solutions from the National Institute of Standards and Technology (NIST; seven DDTs, 41 PCBs) or from reputable suppliers as neat materials with guaranteed purities of at least 99 percent (non-NIST: 48 PCBs, seven phenylalkanes). The only exception to this was $p, p$ '-DDNU which was not available at this purity. In 2006 'research-grade' $p, p$ '-DDNU was purchased from Sigma-Aldrich which, after testing by high resolution gas chromatography/mass spectrometry (HRGC/MS), proved to have a purity of 93.6 percent. Due to time constraints, no attempt was made to further purify this material. Instead, calibration with $p, p$ '-DDNU was performed correcting for the purity estimated by HRGC/MS analysis. During preparation of standard solutions, testing by high resolution gas chromatography/electron capture detection (HRGC/ECD) and/or HRGC/ MS was routinely performed to confirm purities.

\section{Core sectioning}

Cores or core sections were received in the laboratory in a frozen state and were immediately placed into a freezer at $-20{ }^{\circ} \mathrm{C}$. The 124B1-DDT (1992) core was first received from the USGS (Menlo Park, CA) by Arthur D. Little and sectioned at their premises with the device shown in figure 7. Aliquots of the homogenized sections were subsequently sent by Arthur D. Little to the USGS lab in Reston, VA, where they were stored until analysis in 1994. In the case of the 124B1-CH (2003) core, the entire frozen subcore was shipped by the USGS from Menlo Park, CA to Reston, VA; core sectioning was conducted in the Reston, VA, laboratory. 
Detailed procedures used for sectioning frozen cores are provided in appendix 3 . In brief, the frozen core was taken out of the freezer, and the polycarbonate end caps were removed from the liner. The core (in its polycarbonate liner) was then mounted in a sediment extruder developed by the USGS (Reston, VA), with the core top oriented toward the front retention plate (fig. 7). The frozen perimeter of the core was gently defrosted with a heat gun to permit extrusion. The piston of the extruder was then driven into the liner until $2 \mathrm{~cm}$ of the sediment core was exposed out the end of the front retention plate. At this point, canvas rock bags filled with crushed dry ice were draped across the core liner to refreeze the core perimeter during section removal. Any loose ice was carefully removed from the core top at this time. A pre-cleaned hack saw was used to cut off a 2-cm section of the core, which was collected on a piece of pre-cleaned aluminum foil. The outer 0.5 to $1.0 \mathrm{~cm}$ of each sediment section was trimmed with a clean stainless steel blade, after which the inner portion was cut into pieces and transferred to a pre-combusted glass jar. The jar was immediately sealed with a pre-cleaned Teflon ${ }^{\mathrm{TM}}$-lined cap and placed in the freezer. The same procedure was repeated, with cleaning of utensils between sections, until the entire core was sectioned at 2-cm intervals.

\section{Extraction of organic contaminants}

Details of the extraction procedure are given in appendix 4. In brief, frozen sediment sections were brought to room temperature, manually homogenized and weighed into pre-cleaned cellulose Soxhlet extraction thimbles. A separate aliquot was taken for determination of water content. The samples in the thimbles were then amended with small volumes of surrogate solutions corresponding to the chlorinated hydrocarbons (PCB congeners 30, 121 and 198, identified as RS1, RS2, and RS3, respectively) and the LCABs (five 1-phenylalkanes; see below). The sediments were extracted with $\mathrm{MeOH}$ followed by DCM (18 hours at 3 cycles/hour for each solvent). After back extraction of the $\mathrm{MeOH}$ extract with DCM, the extracts were combined and reduced in volume by rotary evaporation. The concentrated extracts were then treated for removal of elemental sulfur (using activated $\mathrm{Cu}^{\circ}$ ) and water (using anhydrous $\mathrm{Na}_{2} \mathrm{SO}_{4}$ ). After volume adjustment, the extracts were analyzed for their content of total extractable organics (TEO) using a Cahn Model 31 microbalance. The extracts were then stored in a freezer until chromatographic fractionation could be performed.

\section{Fractionation of extracts}

Details of the procedure for fractionation of extracts are given in appendix 2. Following is a brief account. Based on results of the gravimetric analysis for TEO (see above), an aliquot of extract equal to $25 \mathrm{mg}$ was transferred to a separate vial. Under a gentle stream of dry nitrogen gas, the solvent was evaporated and replaced with $250 \mu \mathrm{L}$ of hexane. The aliquot was transferred quantitatively to a column consisting of 1:2(v/v) alumina overlying silica gel, both deactivated 3 percent by weight with water. Three fractions were collected: 1) F1, the aliphatic hydrocarbons (eluted with $15 \mathrm{~mL}$ hexane), 2) F2, chlorinated hydrocarbons, LCABs and polycyclic aromatic hydrocarbons (eluted with $5 \mathrm{~mL}$ hexane $+30 \mathrm{~mL} 30$ percent DCM in hexane), and 3) F3, polar compounds (eluted with $40 \mathrm{~mL}$ $\mathrm{MeOH})$. The $\mathbf{F} 2$ fraction was concentrated by rotary evaporation, transferred to a vial and adjusted to a volume of $1 \mathrm{~mL}$ in DCM. It was then divided into three (or four) 'splits' for subsequent instrumental analysis. Splits were stored in the freezer until instrumental analysis could be performed. 


\section{Instrumental analysis}

Elemental abundance

Details of the procedures for determination of total organic carbon (TOC) and total nitrogen $(\mathrm{TN})$ in sediment samples are given in appendix 6. Briefly, sediment samples were thawed, manually homogenized, and sub-sampled. The sub-samples were dried in an oven at $60{ }^{\circ} \mathrm{C}$ and ground to a fine powder. To remove carbonates, a small aliquot was weighed into a silver capsule and exposed to $\mathrm{HCl}$ vapors (Hedges and Stern, 1984). After enclosure of the silver capsule in a tin capsule, the carbonatefree sediments were analyzed on a Carlo Erba EA1108 elemental analyzer. Instrument calibration was performed with acetanilide at five levels. Method detection limits (U.S. Environmental Protection Agency, 1992) were 0.007 percent (TOC) and 0.003 percent (TN). For comparison, concentration ranges of the sediment samples were 0.7 to 3.5 percent (TOC) and 0.05 to 0.28 percent (TN). Accuracy, precision, and continuing calibration verification were established by analysis of a marine sediment standard reference material, PACS-1, from the National Research Council of Canada. PACS-1 has a certified total carbon (TC) content of $3.69 \pm 0.11$ percent, with a non-certified TOC content of 3.56 percent. Replicate analysis $(n=5)$ of PACS-1 resulted in a measured TC content of 3.68 \pm 0.13 percent, a TOC content of $3.53 \pm 0.05$ percent, and a TN content of $0.265 \pm 0.003$ percent. For both the 124B1-DDT (1992) and 124B1-CH (2003) cores, all quality control criteria and objectives set forth in Manen (1994) were met.

\section{Chlorinated hydrocarbons}

Details of the procedures used in the determination of DDTs and PCBs can be found in appendix 7. Following is a brief description of these procedures. The original analysis of the 124B1DDT (1992) core, which took place in 1994, involved determination of seven DDT compounds, namely, the $p, p^{\prime}-$ and $o, p$ '- isomers of DDT, DDD and DDE + $p, p^{\prime}$-DDMU and 84 PCB congeners (table 3). Prior to analysis, the DDT and PCB F2 splits were either evaporated to 'just dryness' under a gentle stream of dry nitrogen gas and immediately taken up in a solution containing known amounts of three internal quantitation standards (tetrachloro-meta-xylene [TCMX], PCB 11, and PCB 207; identified as IS1, IS2 and IS3 in fig. 8) or were diluted beforehand. In either case, final solutions were analyzed using a Hewlett-Packard Series II HRGC equipped with a ${ }^{63} \mathrm{Ni}$ ECD. One-microliter injections onto a $30 \mathrm{~m} \times 0.25 \mathrm{~mm}$ (id) DB-5 fused silica capillary column, $0.25 \mu \mathrm{m}$ film thickness (J\&W Scientific, now part of Agilent Technologies) were made in splitless mode using a HewlettPackard 7673A autosampler. Quantitation was by the internal standard method using PCB 207 (IS3) with seven level multi-point calibration (MPC). HRGC/ECD chromatograms of DDT and PCB calibration standard solutions (panels $\mathrm{A}$ and $\mathrm{C}$, respectively) and representative DDT and PCB F2 splits from the 124B1-CH (2003) core (panels B and D, respectively) are shown in figure 8 . The calibration data were fitted to a quadratic equation with forcing through the origin; all calibrations yielded correlation coefficients $(\mathrm{R})$ greater than 0.995 . Volumes of the final solutions were adjusted to ensure that all analytes fell within their respective calibration ranges and rediluted and reanalyzed if necessary. Calibration was verified on a continuing basis, and blanks, matrix spikes, and standard reference materials were included in the sample analysis stream following specifications similar to those given in Southern California Damage Assessment Analytical Chemistry Quality Assurance Plan (Manen, 1994).

Data were acquired and processed using a Perkin-Elmer Nelson chromatography data system consisting of TurboChrom (v. 3.2) software and a Series 900 LINK interface. Concentrations of the DDTs and PCBs were not corrected for recovery. Identities of the DDTs were confirmed in all 
samples of the 2003 core by electron impact (EI) ionization HRGC/MS analysis of the LCAB F2 splits, whereas identities of both DDTs and PCBs in the 1992 core samples were confirmed by electron capture negative chemical ionization (EC-NCI) MS of selected samples using a Hewlett-Packard MS Engine (5989A) as described by Eganhouse and others (2000). Confirmation of the PCBs in the 2003 core samples by EC-NCIMS has not yet been performed. Because the HRGC/ECD chromatograms of the PCB F2 splits for 1992 and 2003 samples are virtually identical and because there is no indication of transformation of these compounds during this time period (see Polychlorinated biphenyls section), it is reasonable to apply the results of the EC-NCIMS confirmation analyses of 1992 core samples to the 2003 core samples.

The analysis of the 124B1-CH (2003) samples was conducted using the same procedures, standard solutions and instrumentation as described above for the 124B1-DDT (1992) core.

Chromatographic conditions, peak integration, processing parameters and procedures for inspection and manual correction of baseline and peak detection were, likewise, identical with those used in the original 1992 core analyses. The only changes were: 1) the data system was upgraded to PerkinElmer TotalChrom Workstation (v. 6.2.1) software, 2) a Merlin microseal septum (Agilent Technologies) was substituted for a standard silicone rubber septum, and 3) the ECD had been refurbished. One other difference was that shortly before the analysis of the 2003 core began, a sample of synthetic $p, p$ 'DDNU (described in the Materials section) was acquired. After determination of its purity by HRGC/MS, this material was incorporated into a single-point calibration (SPC) standard solution containing the other seven DDT compounds (fig. 8A). This SPC solution was placed in the analytical sequence immediately following the last MPC run and just prior to the first sample run as well as immediately after each 
Table 3. List of target analytes.

\begin{tabular}{|c|c|c|c|}
\hline Chlorinated pesticides $^{\mathrm{a}}$ & Congener number $^{b}$ & Structure & Name $^{a}$ \\
\hline$p, p^{\prime}-\mathrm{DDNU}$ & 1 & 2 & 2-chlorobiphenyl \\
\hline$p, p^{\prime}$-DDMU & 2 & 3 & 3-chlorobiphenyl \\
\hline$o, p^{\prime}-\mathrm{DDE}$ & 3 & 4 & 4-chlorobiphenyl \\
\hline$p, p^{\prime}-\mathrm{DDE}$ & 4 & $2-2$ & 2,2'-dichlorobiphenyl \\
\hline$o, p^{\prime}-\mathrm{DDD}$ & 9 & 25 & 2,5-dichlorobiphenyl \\
\hline$p, p^{\prime}-\mathrm{DDD}$ & 6 & $2-3$ & 2,3'-dichlorobiphenyl \\
\hline$o, p^{\prime}-\mathrm{DDT}$ & 8 & $2-4$ & 2,4'-dichlorobiphenyl \\
\hline \multirow[t]{2}{*}{$p, p^{\prime}-\mathrm{DDT}$} & 19 & $26-2$ & 2,2',6-trichlorobiphenyl \\
\hline & 18 & $25-2$ & 2,2',5-trichlorobiphenyl \\
\hline Long-chain alkylbenzenes & 17 & $24-2$ & 2,2',4-trichlorobiphenyl \\
\hline 5-phenyldecane & 24 & 236 & 2,3,6-trichlorobiphenyl \\
\hline 4-phenyldecane & 16 & $23-2$ & 2,2',3-trichlorobiphenyl \\
\hline 3-phenyldecane & 29 & 245 & 2,4,5-trichlorobiphenyl \\
\hline 2-phenyldecane & 26 & $25-3$ & 2,3',5-trichlorobiphenyl \\
\hline 6-phenylundecane & 25 & $24-3$ & 2,3',4-trichlorobiphenyl \\
\hline 5-phenylundecane & 31 & $25-4$ & 2,4',5-trichlorobiphenyl \\
\hline 4-phenylundecane & 28 & 24-4 & 2,4,4'-trichlorobiphenyl \\
\hline 3-phenylundecane & 33 & $34-2$ & 2',3,4-trichlorobiphenyl \\
\hline 2-phenylundecane & 22 & $23-4$ & 2,3,4'-trichlorobiphenyl \\
\hline 6-phenyldodecane & 46 & $23-26$ & 2,2',3,6'-tetrachlorobiphenyl \\
\hline 5-phenyldodecane & 69 & $246-3$ & 2,3',4,6-tetrachlorobiphenyl \\
\hline 4-phenyldodecane & 52 & $25-25$ & $2,2^{\prime}, 5,5^{\prime}$-tetrachlorobiphenyl \\
\hline 3-phenyldodecane & 49 & $24-25$ & $2,2^{\prime}, 4,5^{\prime}$-tetrachlorobiphenyl \\
\hline 2-phenyldodecane & 47 & $24-24$ & 2,2',4,4'-tetrachlorobiphenyl \\
\hline 7\&6-phenyltridecane & 35 & $34-3$ & 3,3',4-trichlorobiphenyl \\
\hline 5-phenyltridecane & 44 & 23-25 & $2,2^{\prime}, 3,5^{\prime}$-tetrachlorobiphenyl \\
\hline 4-phenyltridecane & 42 & $23-24$ & $2,2^{\prime}, 3,4^{\prime}$-tetrachlorobiphenyl \\
\hline 3-phenyltridecane & 41 & $234-2$ & 2,2',3,4-tetrachlorobiphenyl \\
\hline 2-phenyltridecane & 40 & $23-23$ & 2,2',3,3'-tetrachlorobiphenyl \\
\hline 7-phenyltetradecane & 100 & $246-24$ & 2,2'4,4',6-pentachlorobiphenyl \\
\hline 6-phenyltetradecane & 74 & $245-4$ & 2,4,4',5-tetrachlorobiphenyl \\
\hline 5-phenyltetradecane & 70 & 25-34 & 2,3',4',5-tetrachlorobiphenyl \\
\hline 4-phenyltetradecane & 66 & 24-34 & $2,3^{\prime}, 4,4^{\prime}$-tetrachlorobiphenyl \\
\hline 3-phenyltetradecane & 91 & $236-24$ & 2,2',3,4',6-pentachlorobiphenyl \\
\hline 2-phenyltetradecane & 60 & 234-4 & $2,3,4,4$ '-tetrachlorobiphenyl \\
\hline & 92 & $235-25$ & 2,2 ',3,5,5'-pentachlorobiphenyl \\
\hline & 84 & $236-23$ & $2,2^{\prime}, 3,3^{\prime}, 6$-pentachlorobiphenyl \\
\hline & 101 & 245-25 & $2,2^{\prime}, 4,5,5^{\prime}$-pentachlorobiphenyl \\
\hline
\end{tabular}


Table 3. List of target analytes. - Continued

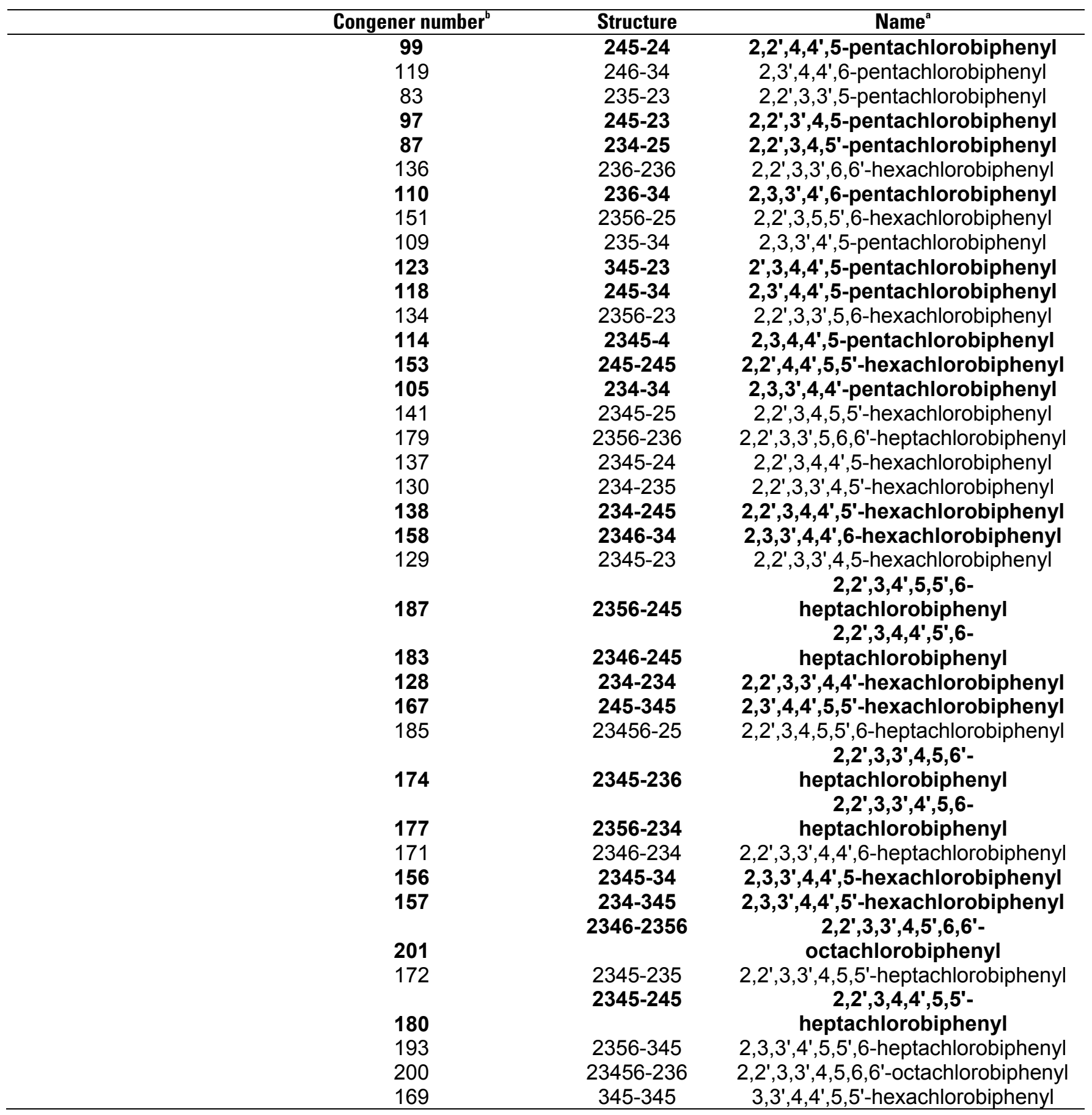


Table 3. List of target analytes. - Continued

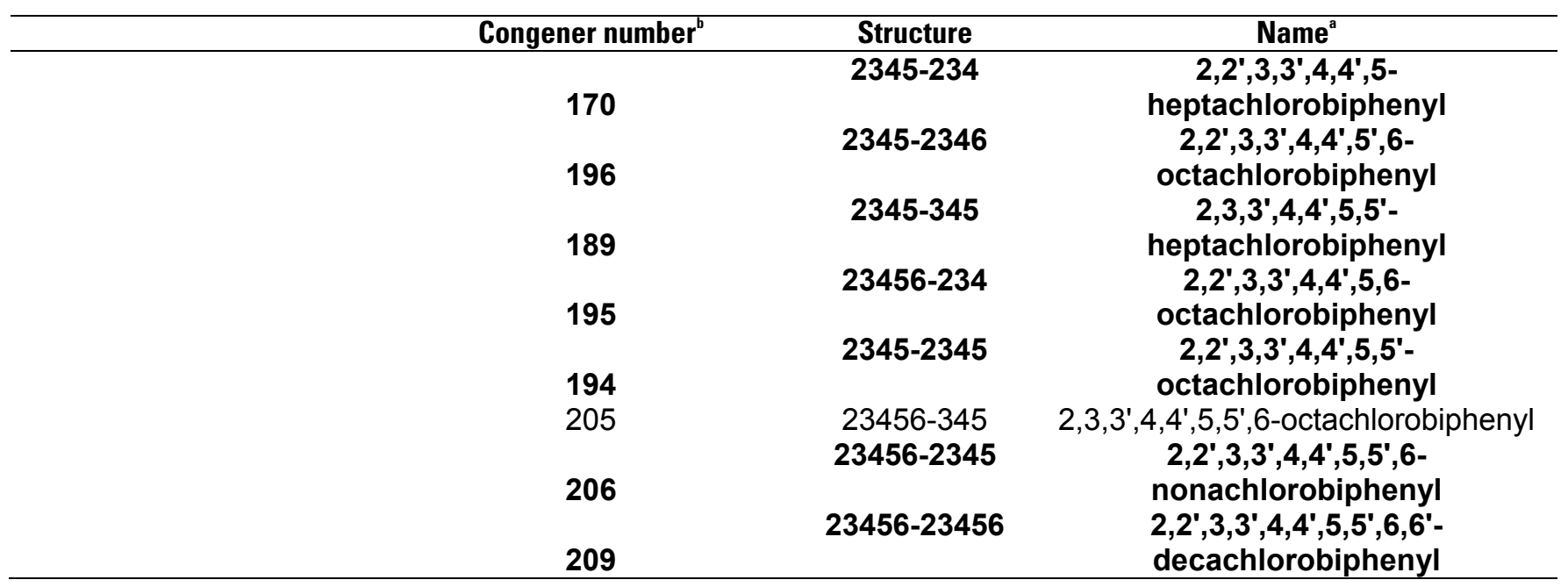

${ }^{a}$ Analytes in bold were specified by NOAA/NIST. Non-bolded analytes were added by the USGS, Reston, VA.

${ }^{\mathrm{b}}$ Congeners numbered according to IUPAC (International Union of Pure and Applied Chemistry). See Guitart and others (1993) for assignments of congeners 109, 200 and 201. 
In the case of the PCBs, a comprehensive evaluation of potential interferences was undertaken, and all data from the 1992 and 2003 cores were reviewed. The reason for this is that in the mid- to late 1990s, following completion of the original analyses of the 1992 core, tremendous advances were made in the analytical chemistry of the PCBs. In particular, an international effort to establish retention time data for all 209 PCB congeners on a large number of stationary phases was undertaken, and all 209 PCB congeners became commercially available for the first time (see references in Cochran and Frame, 1999). Although the comparative nature of the work on the Palos Verdes Remediation Project (PVRP) did not allow all of these improvements to be implemented, newly acquired information concerning the elution of PCB congeners on stationary phases similar to the ones we used in the PVRP was used. The data evaluation involved consideration of the following criteria:

- Known co-elution of the more abundant DDT compounds including the 8 targeted analytes $+p, p$ 'DDMS and $p, p$ '-DDNS (identified by EI-HRGC/MS).

- $\quad$ Evidence of non-DDT interferences with targeted PCB congener peaks based on the EC-NCIMS analyses conducted in 1995 on selected 124B1-DDT (1992) core samples,

- Chromatographic (HRGC/ECD) observations (for example, peak widths and asymmetries),

- Knowledge of the abundance of targeted PCB congeners in Aroclors 1242, 1254 and 1260 (Frame and others, 1996),

- Potential co-elution among PCB congeners based on previous studies in which columns having similar stationary phases were evaluated (Frame, 1997a,b; Cochran and Frame, 1999).

From these considerations, each of the targeted PCB congeners was assigned a score from ' 1 ' to ' 3 ' reflecting the extent of evidence for interference. A score of ' 1 ' indicates that there is no evidence of any interference (34 targeted PCB congeners), a score of ' 2 ' indicates the possibility of interference (41 targeted PCB congeners), and a score of ' 3 ' indicates an interference that was confirmed by mass spectrometry ( 9 targeted PCB congeners). The assignment of a score of ' 2 ' is intentionally conservative and should not be construed as confirmatory of an interference. Based on this analysis, several summed PCB concentration metrics were developed, two of which appear in the discussion to follow. One of these, $\Sigma \mathrm{PCB}_{65}$, refers to the summed concentrations of 65 congener peaks (includes 68 targeted congeners, 3 pairs of which co-elute). This summation comprises all of the congeners assigned a score of ' 1 ' plus some congeners assigned a score of ' 2 ' that are considered likely to be interference-free (table 4). It excludes congeners for which interferences from the aforementioned DDT compounds, TCMX (tetrachloro-meta-xylene) and elemental sulfur are known to exist plus five congeners that have been shown to suffer from unidentified interferences by ECNCIMS. For comparison, in the Southern California Bight Natural Resource Damage Assessment Expert Reports 'Total PCBs' were reported by Arthur D. Little as twice the summed concentration of 18 congeners (\#8, 18, 28, 44, 52, 66, 101, 105, 118, 128, 138, 153, 170, 180, 187, 195, 206, 209; Bailey and Costa, 1994; Lee, 1994a; Manen, 1994). Whole-core inventories of $\Sigma \mathrm{PCB}_{65}$ compared to 'Total PCBs' calculated by this method (using USGS, Reston, VA data) for the 124B1-DDT (1992) and 124B1-CH (2003) cores were 25.8 compared to $27.9 \mathrm{ng} / \mathrm{cm}^{2}$ and $28.9 \mathrm{compared}$ to $31.4 \mathrm{ng} / \mathrm{cm}^{2}$, respectively. This suggests that the directly measured $\Sigma \mathrm{PCB}_{65}$ is reasonably comparable with the estimation technique for 'Total PCBs' used in the Montrose case.

The other summed metric is $\Sigma \mathrm{PCB}_{18}$. This includes 19 of the most abundant congeners spanning a wide chlorination level range $\left(\mathrm{Cl}_{3-9}\right)$ for which no evidence of co-elution exists (table 4; 
NCIMS. For comparison, in the Southern California Bight Natural Resource Damage Assessment Expert Reports 'Total PCBs' were reported by Arthur D. Little as twice the summed concentration of 18 congeners ( $\# 8,18,28,44,52,66,101,105,118,128,138,153,170,180,187,195,206,209$;

Bailey and Costa, 1994; Lee, 1994a; Manen, 1994). Whole-core inventories of $\Sigma \mathrm{PCB}_{65}$ compared to 'Total PCBs' calculated by this method (using USGS, Reston, VA data) for the 124B1-DDT (1992) and $124 \mathrm{~B} 1-\mathrm{CH}$ (2003) cores were 25.8 compared to $27.9 \mathrm{ng} / \mathrm{cm}^{2}$ and 28.9 compared to $31.4 \mathrm{ng} / \mathrm{cm}^{2}$, respectively. This suggests that the directly measured $\Sigma \mathrm{PCB}_{65}$ is reasonably comparable with the estimation technique for 'Total PCBs' used in the Montrose case.

The other summed metric is $\Sigma \mathrm{PCB}_{18}$. This includes 19 of the most abundant congeners spanning a wide chlorination level range $\left(\mathrm{Cl}_{3-9}\right)$ for which no evidence of co-elution exists (table 4; Note: Concentrations of congeners 28 and 31 are summed.). Whereas, $\Sigma \mathrm{PCB}_{65}$ is a reasonable estimate of the total $\mathrm{PCB}$ concentration, $\Sigma \mathrm{PCB}_{18}$ is a reliable quantitative indicator of a significant portion of the total PCBs in a sample. The concentration of $\Sigma \mathrm{PCB}_{18}$ represents $34.4 \pm 1.2$ percent (mean \pm 1 std. dev., $\mathrm{n}=23$ ) of the concentration of $\Sigma \mathrm{PCB}_{65}$ in core 124B1-DDT (1992) and $31.9 \pm 1.1$ percent (mean \pm 1 std. dev., $\mathrm{n}=23$ ) in core 124B1-CH (2003). 
Table 4. Ratings of PCB congener target analytes for potential interferences and inclusion in PCB metrics.

\begin{tabular}{|c|c|c|c|c|}
\hline Congener number & Interference score ${ }^{a}$ & Known interferences & $\Sigma P C B_{55}$ & $\Sigma P B{ }^{b}$ \\
\hline 1 & 2 & & $\checkmark$ & \\
\hline 2 & 2 & $\begin{array}{l}\text { congener not observed in } \\
\text { standard }\end{array}$ & & \\
\hline 3 & 2 & & $\checkmark$ & \\
\hline 4 & 3 & TCMX & & \\
\hline 9 & 2 & & $\checkmark$ & \\
\hline 6 & 2 & & $\checkmark$ & \\
\hline 8 & 2 & & $\checkmark$ & \\
\hline 19 & 1 & & $\checkmark$ & \\
\hline 18 & 1 & & $\checkmark$ & $\checkmark$ \\
\hline 17 & 1 & & $\checkmark$ & $\checkmark$ \\
\hline 24 & 1 & & $\checkmark$ & \\
\hline 16 & 2 & & $\checkmark$ & \\
\hline 29 & 1 & & $\checkmark$ & \\
\hline 26 & 1 & & $\checkmark$ & $\checkmark$ \\
\hline 25 & 2 & & $\checkmark$ & \\
\hline 31 & 1 & & $\checkmark$ & $\checkmark$ \\
\hline 28 & 1 & & $\checkmark$ & $\checkmark$ \\
\hline 33 & 2 & & $\checkmark$ & \\
\hline 22 & 2 & & $\checkmark$ & \\
\hline 46 & 1 & & $\checkmark$ & \\
\hline 69 & 3 & PCB 52 & & \\
\hline 52 & 1 & & $\checkmark$ & $\checkmark$ \\
\hline 49 & 1 & & $\checkmark$ & $\checkmark$ \\
\hline 47 & 2 & & $\checkmark$ & \\
\hline 35 & 2 & & $\checkmark$ & \\
\hline 44 & 1 & & $\checkmark$ & $\checkmark$ \\
\hline 42 & 1 & & $\checkmark$ & $\checkmark$ \\
\hline 41 & 2 & heptachlor? & $\checkmark$ & \\
\hline 40 & 2 & elemental sulfur & & \\
\hline 100 & 3 & $o, p^{\prime}$-DDMU? & & \\
\hline 74 & 1 & & $\checkmark$ & $\checkmark$ \\
\hline 70 & 2 & & $\checkmark$ & \\
\hline 66 & 2 & & $\checkmark$ & \\
\hline 91 & 1 & & $\checkmark$ & \\
\hline 60 & 3 & $p, p^{\prime}$-DDMU & & \\
\hline 92 & 3 & $p, p^{\prime}-\mathrm{DDMU}, o, p^{\prime}-\mathrm{DDE}$ & & \\
\hline 84 & 3 & $o, p^{\prime}-\mathrm{DDE}$ & & \\
\hline 101 & 2 & & $\checkmark$ & \\
\hline 99 & 2 & cis-chlordane? & & \\
\hline 119 & 2 & trans-nonachlor? & $\checkmark$ & \\
\hline 83 & 3 & $p, p^{\prime}$-DDMS & & \\
\hline 97 & 1 & & $\checkmark$ & $\checkmark$ \\
\hline 87 & 2 & & $\checkmark$ & \\
\hline 136 & 3 & $p, p^{\prime}-\mathrm{DDE}$ & & \\
\hline 110 & 2 & $O n^{\prime}-\mathrm{DDD}$ & & \\
\hline
\end{tabular}


Table 4. Ratings of PCB congener target analytes for potential interferences and inclusion in PCB metrics. - Continued

\begin{tabular}{|c|c|c|c|c|}
\hline Congener number & Interference score $^{\mathrm{a}}$ & Known interferences & $\Sigma \mathrm{PCB}_{65}$ & $\Sigma \mathrm{PCB}_{18}$ \\
\hline 151 & 2 & $\begin{array}{l}\text { unknown brominated } \\
\text { compound }\end{array}$ & & \\
\hline 109 & 2 & & $\checkmark$ & \\
\hline 123 & 2 & & $\checkmark$ & \\
\hline 118 & 2 & & $\checkmark$ & \\
\hline 134 & 3 & $p, p^{\prime}-\mathrm{DDD}$, cis-nonachlor? & & \\
\hline 114 & 2 & & $\checkmark$ & \\
\hline 153 & 1 & & $\checkmark$ & $\checkmark$ \\
\hline 105 & 2 & & $\checkmark$ & \\
\hline 141 & 1 & & $\checkmark$ & $\checkmark$ \\
\hline 179 & 1 & & $\checkmark$ & \\
\hline 137 & 1 & & $\checkmark$ & \\
\hline 130 & 2 & $p, p^{\prime}$-DDT & & \\
\hline 138 & 2 & & $\checkmark$ & \\
\hline 158 & 1 & & $\checkmark$ & \\
\hline 129 & 2 & & $\checkmark$ & \\
\hline 187 & 1 & & $\checkmark$ & $\checkmark$ \\
\hline 183 & 1 & & $\checkmark$ & \\
\hline 1287 & 2 & & $\checkmark$ & \\
\hline 167 & 2 & & $\checkmark$ & \\
\hline$\overline{185}$ & 1 & & $\checkmark$ & \\
\hline 174 & 1 & & $\checkmark$ & $\checkmark$ \\
\hline 177 & 1 & & $\checkmark$ & $\checkmark$ \\
\hline 1717 & 2 & & $\checkmark$ & \\
\hline 156 & 2 & & $\checkmark$ & \\
\hline 1577 & 1 & & $\checkmark$ & \\
\hline$\underline{201}\rfloor$ & 1 & & $\checkmark$ & \\
\hline$\overline{172}$ & 1 & & $\checkmark$ & \\
\hline 180 & 1 & & $\checkmark$ & $\checkmark$ \\
\hline 193 & 1 & & $\checkmark$ & \\
\hline 200 & 2 & & $\checkmark$ & \\
\hline 169 & 2 & & $\checkmark$ & \\
\hline 170 & 2 & $\begin{array}{l}\text { possible coelution w/ PCB } \\
190\end{array}$ & & \\
\hline 196 & 1 & & $\checkmark$ & $\checkmark$ \\
\hline 189 & 1 & & & \\
\hline 195 & 2 & & $\checkmark$ & \\
\hline 194 & 2 & & $\checkmark$ & \\
\hline 205 & 2 & & $\checkmark$ & \\
\hline 206 & 1 & & $\checkmark$ & $\checkmark$ \\
\hline 209 & 2 & & $\checkmark$ & \\
\hline
\end{tabular}

${ }^{\mathrm{a}} \mathrm{See}$ text for description of rating system. 1-no interference, 2-possible interference, 3-confirmed interference.

${ }^{b} \mathrm{PCB}$ congeners 28 and 31 were quantified separately in core 124B1-CH but reported as a single peak, whereas these congeners were quantified as a single peak in core 124B1-DDT due to insufficient chromatographic separation. 
Method performance for the 124B1-DDT (1992) core is summarized in Eganhouse and others (2000) and will not be elaborated further here. Owing to time constraints, the same method detection limits (MDLs; U.S. Environmental Protection Agency, 1992) for DDTs and PCBs were used in both the 124B1-DDT (1992) and 124B1-CH (2003) core analyses. With the exception of $o, p$ '- and $p, p$ 'DDT, concentrations of targeted DDT compounds in Palos Verdes Shelf sediments were several orders of magnitude greater than the estimated MDLs. MDLs for all 87 PCBs (including the 3 recovery surrogates) ranged from 0.04 to $16.9 \mathrm{ng} / \mathrm{g}$ as compared to sediment concentrations, which fell between less than 0.02 and $250 \mathrm{ng} / \mathrm{g}$. Concentrations of all analytes (DDTs and PCBs) were less than three times the MDL in all processed blank samples. Duplicate analysis of SRM1941a yielded the following results for the DDTs: $p, p^{\prime}$-DDE-6.71 and $7.04 \mathrm{ng} / \mathrm{g}$ compared to a NIST certified concentration of $6.59 \pm 0.56 \mathrm{ng} / \mathrm{g}, p, p^{\prime}-\mathrm{DDD}-32.4$ and $30.9 \mathrm{ng} / \mathrm{g}$ compared to a certified concentration of $5.06 \pm 0.58 \mathrm{ng} / \mathrm{g}$. Results for the PCB congeners showed that more than half of the 16 PCB congeners listed in table $2 \mathrm{~b}$ of Manen and others (1994) fell within 35 percent of the certified values and 70-75 percent fell within 50 percent of the certified values. (Recoveries of the PCB surrogates in the 124B1-CH (2003) core were as follows: \#30-110 \pm 28 percent, \#121-109 \pm 33 percent, and \#198$109 \pm 30$ percent (mean \pm 1 std. dev.). For the twenty-seven PCB congeners that were spiked within the targeted range (that is, 2 to 10 times the amount in unspiked samples), recoveries in the matrix spike samples were $119 \pm 26$ percent. In the case of the DDT analytes (excluding $o, p^{\prime}$ - and $p, p^{\prime}$-DDT), recoveries in the matrix spike samples were $64 \pm 14$ percent, Precision of the DDT and PCB measurements is estimated at less than 20 percent based on analysis of five replicate samples collected near station 6C (Eganhouse and others, 2000).

\section{Long-chain alkylbenzenes}

Details of the procedures used for determination of the long-chain alkylbenzenes can be found in appendix 5. Following is a brief description of these procedures. Two classes of LCABs were measured in the 1992 and 2003 cores, the linear alkylbenzenes (LABs) and the tetrapropylene-based alkylbenzenes (TABs). The LABs consist of 26 secondary phenylalkanes with alkyl chains having ten to fourteen carbons (fig. 9A). All possible isomers for each chain length are produced during the alkylation process (Swisher, 1987). The LABs are synthesized as precursors to the linear alkylbenzene sulfonates (LAS), the most widely used (anionic) surfactant in commercial detergents. Due to incomplete sulfonation, the LABs occur as minor residues in LAS-containing detergents (Eganhouse and others, 1983a; Takada and Ishiwatari, 1987), which enter waste streams through industrial and domestic detergent consumption (see references in Takada and Eganhouse, 1998). Because of their hydrophobicity (Sherblom and others, 1992), the LABs (and TABs) associate strongly with particles and, thus, find their way to sediments impacted by municipal wastes (Eganhouse and others, 1983b; Ishiwatari and others, 1983). In this setting they have been used as molecular markers of municipal waste for purposes of establishing sedimentation rates through molecular stratigraphy (Eganhouse and Pontolillo, 2000).

The tetrapropylene-based alkylbenzenes were the first LCABs synthesized for production of alkylbenzene sulfonate surfactants (Swisher, 1987; Hons, 1995). They are produced in two steps, polymerization of propylene, and alkylation of benzene. Each step contributes to formation of a complex mixture of alkylbenzenes having branched alkyl chains with 10-15 carbons (fig. 9B). The exact identities of individual components of such $\mathrm{TAB}$ mixtures are not known, but it has been estimated that more than 80,000 congeners are theoretically possible within this chain length range (Ötvös and others, 1973; Swisher, 1987). 
In this study 26 LABs and 12 prominent TAB peaks were measured. The LABs are designated as $\mathrm{j}-\mathrm{C}_{\mathrm{k}}$, where $\mathrm{j}=$ position of substitution of the phenyl group on the alkyl chain, and $\mathrm{k}=$ the number of carbons in the alkyl chain $\left(\mathrm{k}=\mathrm{n}+\mathrm{m}+1\right.$ in fig. 9A). Thus, $6-\mathrm{C}_{12}$ is 6-phenyldodecane. In this report the summed concentrations of the twenty-six LABs is hereafter designated $\Sigma \mathrm{LAB}_{26}$. It should be emphasized that because of possible co-elution, there may be more than one TAB in any given TAB peak. The targeted TAB peaks were assigned numbers 1 through 12 for consistency with previous reports (Eganhouse and others, 1983b; Eganhouse and others, 2000; Eganhouse and Pontolillo, 2000). The summed concentrations of the twelve targeted TAB peaks is hereafter designated $\Sigma T \mathrm{~TB}_{12}$. Sediment samples were spiked with long-chain alkylbenzene surrogates consisting of 1-phenylalkanes having alkyl chain lengths from 10 to 14 (1- $\mathrm{C}_{10-14}$; see fig. 9A for elution order). These compounds have physical-chemical properties very similar to both the LABs and TABs (Sherblom and others, 1992).

Procedures for the analysis of the LCABs in the 124B1-DDT (1992) core are described in Eganhouse and others (2000). The principle changes implemented during analysis of the 124B1-CH (2003) core were: 1) a different HRGC/MS system was used, 2) injections were made in splitless mode with an autosampler rather than manually in on-column mode, and 3) qualifier ions were implemented to increase confidence in analyte peak identifications. Following is a brief description of the procedures used for the 124B1-CH (2003) core analyses.

Prior to analysis, the LCAB F2 splits were transferred to autosampler vials fitted with glass 'micro' inserts. The solutions were evaporated to 'just dryness' under a gentle stream of dry nitrogen gas and immediately taken up in a solution containing known amounts of two internal quantitation standards (1-C 9 and 1- $\mathrm{C}_{15}$-see fig. 9-10). The samples were immediately analyzed using an Agilent 6890 HRGC interfaced to an Agilent 5973 mass selective detector. One-microliter injections onto a 30 $\mathrm{m} \times 0.25 \mathrm{~mm}$ (id) DB-5 fused silica capillary column, $0.25 \mu \mathrm{m}$ film thickness (Agilent Technologies) were accomplished in splitless mode using an Agilent 7683 autosampler. Data acquisition and processing were performed with Agilent ChemStation (D01.00 Build 75) software. Quantitation was by the internal standard method with five-level MPC (LABs). The LAB MPC data were fitted to a linear equation with forcing through the origin. Correlation coefficients $(\mathrm{R})$ for the calibration curves exceeded 0.995 for all analytes. In the case of the TABs, single-point calibration was used because of the similarity in chromatographic and mass spectrometric behavior of the LABs and TABs and the excellent linear calibrations achieved with the LAB MPCs. HRGC/MS total ion current (TIC) chromatograms of TAB and LAB calibration standard solutions (panels A and B, respectively) along with mass fragmentograms of the LCAB F2 splits for a section from the 124B1-CH (2003) core (panels C-E, respectively) are shown in figure 10. Data were acquired in the full scan EI mode (50$500 \mathrm{amu}$ at $1.68 \mathrm{~Hz}$ ) with quantitation based on carefully selected target ions and three qualifier ions for confirmation (see appendix 5). This was necessitated by the generally low concentrations of the LABs, the presence of other non-target LCABs in the sediments and overlapping elution patterns of the LABs and TABs (fig. 10; see also Eganhouse and others, 1983a; Zeng and Yu, 1996; Zeng and others, 1998).

Method performance for the LAB analyses of core 124B1-DDT (1992) is described in Eganhouse and others (2000) and will not be discussed further here. Again, owing to time constraints, MDLs that had been developed during the 1992 core analyses were used for the LABs in the 124B1$\mathrm{CH}$ core. MDLs for individual LABs ranged from 0.5 to $10.7 \mathrm{ng} / \mathrm{g}$ (mean $\pm 1 \mathrm{std}$. dev. $=5.3 \pm 2.8$ $\mathrm{ng} / \mathrm{g}$ ), whereas concentrations in sediment samples varied from less than 0.03 to $88 \mathrm{ng} / \mathrm{g}$.

Concentrations of all analytes were less than three times the MDL in all processed blank samples. Recoveries of the LAB surrogates in the $124 \mathrm{~B} 1-\mathrm{CH}$ (2003) core were as follows: $1-\mathrm{C}_{10}-97 \pm 15$ 
percent, $1-\mathrm{C}_{11}-91 \pm 9$ percent, and $1-\mathrm{C}_{12}-111 \pm 12$ percent, $1-\mathrm{C}_{13}-108 \pm 10$ percent, $1-\mathrm{C}_{14}-104 \pm 8$ percent (mean \pm 1 std. dev.). For this comparative study, concentrations of the LABs and TABs were not corrected for recovery. Total LAB concentrations $\left(\Sigma \mathrm{LAB}_{26}\right)$ determined in this study and corrected for 2 percent moisture, in SRM 1941a were 2.86 and $3.00 \mu \mathrm{g} / \mathrm{g}$ as compared with an average for two outside laboratories of $2.98 \pm 0.05 \mu \mathrm{g} / \mathrm{g}$ (Hartmann and others, 2000). Precision is estimated at less than 12 percent based on analysis of five replicate surficial sediment samples collected near station 6C (Eganhouse and others, 2000; Hendricks and Eganhouse, 1992).

\section{QA/QC procedures}

Procedures for quality assurance/quality control observed during analysis of the 1992 core (124B1-DDT) were based on guidelines developed during the field studies in support of the Montrose case. The specific QA/QC requirements of the program are laid out in Manen (1994). Analyses performed in Reston, VA, had some variations because the analytical work was being done at the same time the QA/QC plan was under development and the fact that additional analytes and surrogates, not covered in the plan, were quantified in these studies. In the course of these earlier analyses it was discovered that the standard reference material that was being developed by NIST for the DDTs and PCBs in the Montrose case, SRM 1941a, contained comparable concentrations of these two analyte groups (Schantz and others, 1995), unlike Palos Verdes Shelf sediments whose DDT concentrations greatly exceed PCB concentrations. The significance of this is that quantitation of minor DDT compounds in the SRM (such as $p, p$ '-DDD) is more difficult than it is in Palos Verdes Shelf sediments. Moreover, the F2 fraction produced for the SRM was considerably more complex than that of Palos Verdes Shelf sediment samples. This resulted in problems with quantitation of two DDT compounds (not $p, p$ '-DDE) and some PCB congeners in the SRM and, in particular, quantitation of one of the recovery surrogates, PCB 198, which suffered from interference in SRM 1941a-derived F2 fractions. This recovery problem did not arise for the Palos Verdes Shelf sediment samples; recoveries for PCB 198 in the 1992 core samples fell within quality control criteria in all cases $(99.9 \pm 20.2$ percent; mean \pm 1 std. dev.). These caveats aside, other QA/QC requirements of the program were met in the analyses of the 1992 core as described in Eganhouse and others (2000) and Eganhouse and Pontolillo (2000).

For purposes of comparability, the same QA/QC procedures were adopted during the analysis of the 2003 core as had been used for the 1992 core, including incorporation of SRM1941a in the sample processing train, despite the aforementioned difficulties with this material. One benefit of doing this was that in the interim two laboratories had published results of their analysis of SRM1941a for the LABs (Hartmann and others, 2000). This provided a means of assessing the accuracy of LAB measurements in the present study, something that was not possible when the 1992 core was originally analyzed.

\section{Computations}

In this section methods used for computing chemical inventories in cores, aligning cores according to chemical concentration/inventory profiles, estimating first-order degradation rates, and modeling the reductive dechlorination kinetics of $p, p^{\prime}$-DDE and $p, p$ '-DDMU are described. Procedures were chosen to maximize comparability among studies that have been done on the Palos Verdes Shelf. 


\section{Inventories}

Lee (1994a) presented whole-core inventories (also referred to as 'cumulative mass per unit area') of $p, p$ '-DDE on the Palos Verdes Shelf based on LACSD data (1981 to 1991) and $p, p$ '-DDE, total DDT (sum of 7 DDT compounds), 'Total PCBs' (2 times the sum of 18 PCB congeners; see above), and TOC based on USGS core data (1992). In the case of the 1992 USGS cores, bulk densities derived from the core logger at $1-\mathrm{cm}$ intervals were used to calculate dry densities as follows.

$$
\delta_{d r y}=\delta_{\text {grain }}\left(\delta_{\text {bulk }}-\delta_{w}\right) /\left(\delta_{\text {grain }}-\delta_{w}\right)
$$

Where: $\quad \delta_{d r y}=$ dry density of sediments $\left(\right.$ dry $\left.\mathrm{g} / \mathrm{cm}^{3}\right)$,

$\delta_{\text {grain }}=$ density of sediment grains $\left(\mathrm{g} / \mathrm{cm}^{3}\right)$,

$\delta_{\text {bulk }}=$ bulk density of wet sediments $\left(\mathrm{g} / \mathrm{cm}^{3}\right)$,

$\delta_{w}=$ density of water $\left(\mathrm{g} / \mathrm{cm}^{3}\right)$.

For purposes of the computations made in this project, an in situ water density of $1.0 \mathrm{~g} / \mathrm{cm}^{3}$ and a grain density $\left(\delta_{\text {grain }}\right)$ of $2.65 \mathrm{~g} / \mathrm{cm}^{3}$ were assumed. To compute average dry densities for each $2-\mathrm{cm}$ section, dry densities for the depth interval mid-point, $\bar{\delta}_{d r y, x}$, and the overlying and underlying $1-\mathrm{cm}$ depth intervals were weighted and summed:

$$
\bar{\delta}_{d r y, x}=\frac{\delta_{d r y, x-1}}{4}+\frac{\delta_{d r y, x}}{2}+\frac{\delta_{d r y, x+1}}{4}
$$

Where: $\quad \delta_{d r y, x}=$ dry density of sediments at interval mid-point, $x\left(\right.$ dry $\left.\mathrm{g} / \mathrm{cm}^{3}\right)$, $\delta_{d r y, x-1}=$ dry density of sediments at interval $1 \mathrm{~cm}$ above mid-point $\left(\mathrm{g} / \mathrm{cm}^{3}\right)$, $\delta_{d r y, x+1}=$ dry density of sediments at interval $1 \mathrm{~cm}$ below mid-point $\left(\mathrm{g} / \mathrm{cm}^{3}\right)$.

Chemical inventories $\left(I_{\text {sect }} ;\right.$ mass $\left./ \mathrm{cm}^{2}\right)$ within each section were obtained as the product of the concentration of chemical $i, C_{i}$ (mass/dry g), the average dry density at each section midpoint, $\bar{\delta}_{d r y, x}\left(\mathrm{dry} \mathrm{g} / \mathrm{cm}^{3}\right)$, and section length, $l_{\text {sect }}(\mathrm{cm})$ :

$$
I_{\mathrm{sect}}=C_{i} \bar{\delta}_{d r y, x} l_{\mathrm{sec} t}
$$

The whole-core inventory $\left(I_{\text {core }} ;\right.$ mass $\left./ \mathrm{cm}^{2}\right)$ is simply the sum of the inventories in each section, $I_{\text {sect }}$.

Another useful measure is the compaction-corrected depth $\left(Z_{c} ; \mathrm{g} / \mathrm{cm}^{2}\right)$. This represents the dry mass of sediment per unit area accumulated to a specified depth below the sediment-water interface. It is obtained as the cumulative products of the average dry densities of each section $i, \bar{\delta}_{d r y, i}\left(\mathrm{dry} \mathrm{g} / \mathrm{cm}^{3}\right)$ and section length, $l_{\text {sec } t, i}(\mathrm{~cm})$ :

$$
Z_{c}=\sum_{i=1}^{n} \bar{\delta}_{d r y, x_{i}} l_{\sec t_{i}}
$$

Where: $\quad n=$ total number of sections. 
In the case of stations where combined box/gravity core pairs were required to capture the history of DDT emissions (Lee and others, 2002), data for bulk densities and chemical concentrations of overlapping intervals were weighted to obtain inventories. An example of a box core/gravity core combination profile (station 556; fig. 5A) with one overlapping interval is shown in figure 11. To check these calculations, computed inventories were compared with those reported by Lee (1994a). The relative percent difference (RPD) ranged from 0 to 1.5 percent (average $=0.7$ percent) for cores 124B1-DDT, 127B1, 147B3/179G3, 169B1/177G2, and 171B1/181G2. The small differences between the inventories calculated in Reston, VA, and those reported by Lee (1994a) are considered trivial and are probably due to slight variations in computation of inventories for overlapping box/gravity core sections.

\section{Core alignment}

In order to compare chemical concentration/inventory data in cores collected from the same location but at different times, a statistical core alignment procedure, briefly described in Eganhouse and Pontolillo (2000), was used. The purpose of this procedure is to adjust the vertical alignment of two cores so that the corresponding depth intervals are of the same approximate age (coeval). The assumption is that during the period between core collection, intense mixing is constrained to a limited portion of the upper sediment column and that the vertical concentration/inventory profiles in deeper subsurface sediments have largely been preserved. This requires that diagenetic reactions, which could lead to alteration of the vertical concentration/inventory profiles, are negligible. These assumptions and evidence in support of them will be discussed in greater detail in a later section.

The alignment procedure consists of three steps: 1) computation of compaction-corrected depths (see discussion above), 2) calculation of chemical concentrations at $1-\mathrm{g} / \mathrm{cm}^{2}$ (or $1-\mathrm{cm}$ ) depth intervals using linear interpolation, 3) correlation analysis (Pearson, pairwise) of interpolated concentration data with depth offsets varying by increments of $1 \mathrm{~g} / \mathrm{cm}^{2}$. The core offset that yields the highest correlation coefficient is taken as the amount of net sediment accumulation in $\mathrm{g} / \mathrm{cm}^{2}$ during the period between core collection. Analysis was carried out using SYSTAT v. 11 software with a range of depth offsets from -20 to $+16 \mathrm{~g} / \mathrm{cm}^{2}$ (or cm; 2003 compared to 1992). Data used in the analysis included measured concentrations of $p, p^{\prime}$-DDE, $p$, $p$ '-DDE $+p$, $p^{\prime}$-DDMU, 6-C 12 , TAB3 (also referred to as TAB peak \#8; fig. 9B), PCB 153, and $\Sigma \mathrm{PCB}_{65}$. As will be discussed, TAB3, $\Sigma \mathrm{PCB}_{65}$, and, in particular, PCB 153 were considered conservative geochemical tracers that are unaffected by diagenetic transformation processes at this site. For this reason, direct core-to-core calculations of first-order transformation rates were based on offsets derived from vertical distributions of these compounds.

An example of the results of the core alignment procedure using data for the 124B1-DDT (1992) and 124B1-CH (2003) cores is shown in figure 12. Variations among the profiles for a given graph reflect differences in: 1) the persistence of the compounds during the time between core collection, and 2) the vertical distribution of these substances as controlled by historical inputs to the sediments. Vertical concentration profiles of PCB 153 before and after alignment using the optimal offset determined for this compound by the core alignment procedure $\left(4-\mathrm{g} / \mathrm{cm}^{2}\right.$ offset) are shown in figure 13. Comparison of figures 13A and 13B suggests that even relatively minor details of the vertical concentration profiles are properly aligned for this core pair. However, there is still some uncertainty in the optimal offset. This can be attributed to an inability to define downcore variations in the profiles when sectioning at 2-cm depth intervals, in other words, inadequate spatial resolution. One means of verifying the offset is to construct cumulative inventory (CI) profiles as shown in figure 14. This allows one to examine the effect of different offsets on the correspondence in CI profiles for 
deeper portions of the sediment column where redistribution processes (for example, bioturbation, resuspension) are expected to be of limited or no importance. CI profiles for the 124B1 cores with no offset are shown in figure 14A. Offsets of $3-4 \mathrm{~g} / \mathrm{cm}^{2}$, as shown in figure $14 \mathrm{~B}$, provide reasonable matches for the CI profiles at depths where the inventory of PCB 153 tends to increase most rapidly with depth (approximately 30 to $40 \mathrm{~g} / \mathrm{cm}^{2}$ ). Based on an offset of $4 \mathrm{~g} / \mathrm{cm}^{2}$ over the 11.07 years between core collection, a net sediment accumulation rate near station $3 \mathrm{C}$ of $0.36 \mathrm{~g} / \mathrm{cm}^{2}$-yr is obtained. Estimates for station $3 \mathrm{C}$ based on regression analysis of LACSD data (whole-core inventories) for the period 1991 to 2005 yield a net sediment accumulation rate of $0.52 \pm 0.14 \mathrm{~g} / \mathrm{cm}^{2}$-yr (C.R. Sherwood and B. Ferré, written commun., 2007). Given the uncertainties involved, differences in the time periods, and the fact that the cores used to develop these estimates come from slightly different locations, the rates are in reasonably good agreement.

\section{Transformation kinetics}

To properly estimate $p, p$ '-DDE transformation rates, it is necessary to determine the order of the reaction, and this can only be done from field or laboratory data. Initial efforts in the present study to determine reaction order centered on an analysis of single core data sets. Downcore increases in the relative abundance of $p, p$ '-DDMU compared to $p, p$ '-DDE were taken as evidence of reductive dechlorination, whereas molecular stratigraphy was used to determine average ages of sediment layers. This analysis depends on assumptions that: 1) $p, p^{\prime}$-DDMU is a dead-end product, 2) the initial $p, p$ 'DDE content of the sediments within any given layer can be estimated as the sum of $p, p$ '-DDE and $p, p$ '-DDMU concentration in that layer, and 3) dehydrochlorination of $p, p$ '-DDD to $p, p$ '-DDMU is unimportant (Quensen and others, 1998). With the discovery of measureable quantities of $p, p$ '-DDNU in the 1992 and 2003 cores and increasing whole-core inventories of this compound between 1992 and 2003 (analogous to $p, p$ '-DDMU), a critical assumption of the analysis was deemed invalid. Consequently, this approach was abandoned and attention was directed to existing experimental data.

Quensen and others (1998) conducted laboratory experiments in which sediments collected from three stations on the Palos Verdes Shelf (3C, 5C, 8C; fig. 3B) were spiked with ${ }^{14} \mathrm{C}$-labelled $p$, $p$ 'DDE and allowed to incubate at room temperature for 32 weeks. Two treatments were used, sulfidogenic and methanogenic, and samples were sacrificed at 8 week intervals. Although raw data for the experiments were not provided, figure 2 in this publication shows the mass balance results at station $3 \mathrm{C}$ for the methanogenic treatment. Data for the ${ }^{14} \mathrm{C}$-DDE activity were read from this chart and used to evaluate reaction order. During the first 8 weeks $p$, $p$ '-DDE loss exhibited a lag period; therefore, only measurements made at 16, 24 and 32 weeks were examined. The data were analyzed using the van't Hoff method with an approximation for the differential term $(d C / d t \approx \Delta C / \Delta t)$ as follows:

$$
\log \left(-\Delta C_{D D E}\right) \approx k+n \log \bar{C}_{D D E}
$$

Where: $\quad \Delta C_{D D E}=$ difference in per cent ${ }^{14} \mathrm{C}$ - DDE activity remaining during successive sampling times,

$k=\mathrm{a}$ constant, $n=$ order of reaction, $\bar{C}_{D D E}=$ average ${ }^{14} \mathrm{C}$ - DDE activity during successive sampling times. 
A plot of $\log \left(-\Delta C_{D D E}\right)$ versus $\log \bar{C}_{D D E}$ yielded a slope of 1.49 , but only two data points could be included. Addition of the data for the 8 week sampling period resulted in a negative slope and a very poor correlation coefficient.

A similar analysis was attempted with data published by Quensen and others (2001) who performed microcosm experiments similar to those described in Quensen and others (1998). P,p'DDMU concentrations plotted in figure 3 of Quensen and others (2001) for sulfidogenic and methanogenic experiments at $15^{\circ} \mathrm{C}$ were transformed to DDE concentrations and plotted in a van't Hoff diagram as described above. In this case, $\log \left(-\Delta C_{D D E} / \Delta t\right)$ was plotted versus $\log \bar{C}_{D D E}$ to account for the non-uniform sampling intervals. Because of variability in the experimental data, the results were unsatisfactory (negative slopes) and, thus, did not support a determination of reaction order. Some of the variability that plagued these experiments may be due to the very crude method of separating $p, p^{\prime}$-DDE from $p, p^{\prime}$-DDMU (thin layer chromatography) and the fact that the purities of the separated fractions were not confirmed by HRGC/MS.

The only other microcosm data available for Palos Verdes Shelf sediments are those of Deming and Carpenter (2000) who conducted incubations of cores collected at stations 3C, 6C and 8C in 1997. Without going into details of the experimental design, the data developed in this study did not support determination of reaction order because measurements were made only at the beginning and end (55 weeks) of the experiments. Therefore, in lieu of any strong direct field or experimental evidence of reaction order and for the sake of maintaining consistency with calculations being made with LACSD core data (C.R. Sherwood and B. Ferré, written commun., 2007), it was decided to develop estimates of the rate coefficients for the core data assuming a reaction order of one.

First-order transformation rates for aligned core intervals were made using the equation:

$$
\lambda=\left\{\ln \left(\frac{[D D E]_{1992}}{[D D E]_{2003}}\right)\right\} / t
$$

Where: $\quad[D D E]_{1992}=$ concentration of $p, p$ '-DDE in sediment of the 1992 core $(\mu \mathrm{g} / \mathrm{dry} \mathrm{g})$, $[D D E]_{2003}=$ concentration of $p, p$ '-DDE in sediment of the 2003 core $(\mu \mathrm{g} / \mathrm{dry} \mathrm{g})$, $t=$ time between core collection (yr).

Assumptions inherent in these calculations include: 1) transformation of $p$, $p$-DDE follows first-order kinetics, 2) aligned core intervals are coeval, 3) differences between concentrations at a given interval pair are due solely to transformation processes, and 4) there are no diagenetic sources of $p, p$-DDE.

The first assumption has already been discussed. Concerning the second assumption, evidence to be provided in a later section will show that the PCBs and some of the TABs (for example, TAB3) behave conservatively, whereby whole-core inventories of these substances in the 1992 and 2003 cores are identical (within analytical uncertainty). Moreover, there is no evidence of reductive dechlorination of the PCBs within or between these cores. Under these circumstances, core alignment based on the distributions of these compounds should, in principle, result in alignment of sediment layers having the same average age. The exception to this could occur within the upper mixed layer where sedimentation, resuspension and mixing all serve to modify the average age of the sediments and associated contaminants. Thus, assumption 2 applies best to sediments deposited below the mixed layer of the most recently collected core. Based on the abundance of benthic infauna and ${ }^{234} \mathrm{Th}$ data (Stull and others, 1996; Wheatcroft and Martin, 1996; Santschi and others, 2001) it appears that the 
mixed layer near station $3 \mathrm{C}$ is on the order of a few centimeters. Evidence in support of assumption 3 is as follows. Because of similarities in the whole-core inventories of the PCBs and some TABs, differences in the amounts of other contaminants, such as $p, p$ '-DDE and $p, p$ '-DDMU, between aligned core intervals must be due to in situ transformation. With the exception of freshly deposited sediments, this can even be extended to the surficial layers of the sediment column because the physical-chemical properties of $p, p$ '-DDE are very similar to those of PCB 153 (Li and others, 2003; Shen and Wania, 2005). Consequently, physical-chemical processes effecting transport of PCB 153 within the sediments (resuspension, mixing, pore water advection, diffusion) or between sediments and water (diffusion, sorption/desorption) should be the same for $p, p^{\prime}$-DDE. Differences in $p$, $p$ '-DDE concentrations between aligned intervals of the upper sediment column must, therefore, be due primarily to diagenetic transformation. As evidence of this, figure 13B shows that PCB 153 concentrations are essentially identical in surficial sediments of both cores, whereas the $p, p$ '-DDE concentrations are reduced in the 2003 core (see below). Finally, it is safe to assume that there are no diagenetic sources of $p, p^{\prime}$-DDE within the sediments because $p, p$ '-DDT is the only possible precursor (fig. 4). Concentrations of $p, p$ '-DDT, even in the 1992 core, were very low, in some cases below the MDL, and averaged less than 1 percent of $p, p$ '-DDE. Consequently, there is insufficient parent $p, p^{\prime}-$ DDT to supply $p, p$ '-DDE to the system. Even if there was an adequate supply, dehydrochlorination within anoxic layers of the sediments, which are just below the top couple of centimeters (Van Cappellen and Santschi, 1999), would not be favored.

\section{Modeling multi-step transformation kinetics}

The average rate at which $p, p$ '-DDE was reductively dechlorinated to $p, p$ '-DDMU at station 522 from 1992 to 2003 was determined through a direct comparison of the 124B1 cores following alignment. Identification and quantitation of $p, p^{\prime}$-DDMU and $p, p^{\prime}$-DDNU in these cores provided additional data with which to estimate rates of: 1) reductive dechlorination of $p, p$ '-DDMU to $p, p$ 'DDNU, and 2) possible transformation of $p, p$ '-DDNU to as-yet-unidentified products. If one assumes that a chain of three reactions proceeds by first-order kinetics and that the rate constant of the first reaction is known, the amounts of the parent and products of these reactions at two or more times can be used to estimate the rate constants of the subsequent reactions. The solution for such a chain of reactions was discovered by Bateman (1910) who investigated the kinetics of multi-step radioactive decay series.

In the case of a single parent ( $p, p^{\prime}$-DDE, $\left.n=1\right)$ and two successive products ( $p, p^{\prime}$-DDMU, $n=2$; $p, p$ '-DDNU, $n=3)$, all of which are present at $t=0$, the amount of $p, p$ '-DDE, $N_{l}$, at time $t$ is given by:

$$
N_{1}=N_{1}^{0} e^{-\lambda_{1} t}
$$

Where: $\quad N_{n}^{0}=$ amount of chemical $n$ at $t=0$,

$\lambda_{i}=$ first-order transformation rate of reaction $i\left(\mathrm{yr}^{-1}\right)$,

$t=$ time (yr).

$p, p$ '-DDMU is formed at the rate at which $p, p^{\prime}$-DDE is dechlorinated and is itself dechlorinated to $p, p$-DDNU at a rate of $\lambda_{2} N_{2}$. It can be shown that the solution of this two-step reaction sequence for the amount of $p, p$-DDMU at time $t$ is given by: 


$$
N_{2}=\frac{\lambda_{1}}{\lambda_{2}-\lambda_{1}} N_{1}^{0}\left(e^{-\lambda_{1} t}-e^{-\lambda_{2} t}\right)+N_{2}^{0} e^{-\lambda_{2} t}
$$

The Bateman solution for the three-step reaction sequence,

$\left(\mathrm{DDE} \stackrel{\lambda_{1}}{\rightarrow}\right.$ DDMU $\stackrel{\lambda_{2}}{\rightarrow}$ DDNU $\stackrel{\lambda_{3}}{\rightarrow}$ unknown) gives the amount of $p, p$ '-DDNU at time $\mathrm{t}$ as:

$$
\begin{aligned}
& N_{3}=\frac{\lambda_{1} \lambda_{2} N_{1}^{0}}{\left(\lambda_{2}-\lambda_{1}\right)+\left(\lambda_{3}-\lambda_{1}\right)} e^{-\lambda_{1} t}+\left[\left(\frac{\lambda_{1} \lambda_{2} N_{1}^{0}}{\left(\lambda_{1}-\lambda_{2}\right)\left(\lambda_{3}-\lambda_{1}\right)}\right)+\left(\frac{\lambda_{2} \lambda_{3} N_{2}^{0}}{\lambda_{2}-\lambda_{2}}\right)\right] e^{-\lambda_{2} t}+ \\
& {\left[\left(\frac{\lambda_{1} \lambda_{2} N_{1}^{0}}{\left(\lambda_{1}-\lambda_{3}\right)\left(\lambda_{2}-\lambda_{3}\right)}\right)+\left(\frac{\lambda_{2} \lambda_{3} N_{2}^{0}}{\lambda_{2}-\lambda_{3}}\right)+N_{2}^{0}\right] e^{-\lambda_{3} t} }
\end{aligned}
$$

Measured inventories of $p, p^{\prime}$-DDE, $p$, $p^{\prime}$-DDMU, and $p, p^{\prime}$-DDNU in the 1992 core were used as input values for $N_{1}^{0}, N_{2}^{0}$, and $N_{3}^{0}$. The mean value of the rate constants for all intervals of the aligned 124B1 cores, $0.052 \mathrm{yr}^{-1}$, was used for $\lambda_{1}$. Optimal values for $\lambda_{2}$ and $\lambda_{3}$, were determined by adjusting the rate constants (in succession) until inventories calculated for 2003 matched those measured in the 124B1-CH (2003) core. Insofar as the rate constants calculated for each reaction are dependent on the accuracy of those of preceding reactions, uncertainty is propagated at each step along the reaction pathway in this optimization procedure. Uncertainty is governed principally by the validity of the assumptions and the accuracy of the measured inventories.

\section{Spatial distribution of DDE, DDMU}

This section covers the distribution of the major DDT transformation products in sediments on the Palos Verdes Shelf. For that purpose, data produced as part of the LACSD ocean monitoring program, the USGS studies in support of the Montrose case, and the sediment displacement studies by SAIC are examined. One line of evidence that reductive dechlorination is occurring on the Palos Verdes Shelf is the increasing abundance of $p, p^{\prime}$-DDMU (relative to $p, p$ '-DDE) with depth in sediment cores (see fig. 2-3). One could hypothesize that this trend is simply a reflection of historical variation in DDT composition of waste inputs, but identical profiles are not found at all stations or in replicate cores at the same station as would be expected. Moreover, the recent increase in $p, p$ '-DDMU abundance in sediments at stations $3 \mathrm{C}$ and $6 \mathrm{C}$ supports the concept that reductive dechlorination of p,p'-DDE is an ongoing diagenetic process. Data for cores collected in 1992 (USGS), in 1991 and 2005 (LACSD) and in 2004 (SAIC) can be used to examine the spatial variability of these downcore trends. In some cases, replicate cores were collected (LACSD-2005). In other cases, sampling was conducted at the same time and place using different corers (SAIC-2004) or the same cores were analyzed by more than one laboratory (USGS, Reston/Arthur D. Little-1992). Although limited, these data provide a qualitative means of assessing the spatial distribution of $p, p$ '-DDMU and $p, p^{\prime}$-DDE on the Palos Verdes Shelf and the influence of sampling and analytical methodology.

Plots of the percent $p, p$ '-DDMU (relative to $p, p^{\prime}$-DDMU $+p, p$ '-DDE) and vertical concentration profiles for the sum, $p, p^{\prime}$-DDMU + p, $p$ '-DDE, in cores collected by the USGS in 1992 are shown in figure 15. On average, the sum, $p, p^{\prime}-\mathrm{DDMU}+p, p^{\prime}$-DDE, in these cores represents 76.4 to 84.9 percent of the total DDT $\left(\sum \mathrm{DDT}_{\mathrm{n}=7}\right)$, the rest effectively being made up by $o, p$ '-DDE and the two isomers of DDD. For this reason, vertical concentration profiles of $p, p^{\prime}$-DDMU $+p, p$ '-DDE are 
considered reliable representations of the distribution of total DDT (see also Eganhouse and others, 2000). The upper panels in figure 15 show data produced by the USGS (Reston, VA) and the Montrose case contractor, Arthur D. Little. In general, $p, p$ '-DDMU abundance is around 20 percent in the upper $30 \mathrm{~g} / \mathrm{cm}^{2}$ of both profiles, increasing irregularly below that with increasing depth. The higher abundances obtained by Arthur D. Little deeper in the core (greater than $40 \mathrm{~g} / \mathrm{cm}^{2}$ ) are most likely due to increased uncertainties of measuring low concentrations. Because these data sets were produced by two labs using different methodologies but the same core samples, they offer a view of the contribution inter-laboratory analytical variability might be expected to make to results obtained by different programs. With the exception of the 169B1-177G2 core pair (fig. 15E), the other profiles generally have lower $p, p^{\prime}$-DDMU abundances in the upper sections (approximately 10 to 15 percent) with variable or increasing abundances at greater depth (approximately 15 to at least 20 percent). This suggests that reductive dechlorination is an ongoing diagenetic process.

For comparison, profiles for gravity cores collected by the LACSD in 1991 at stations 3C and $6 \mathrm{C}$ are given in figures $2 \mathrm{~A}$ and $3 \mathrm{~A}$. [Note: USGS station 522 is near LACSD station 3C and USGS station 556 is near LACSD station 6C (compare fig. 5A-5B).] Using the $p, p$ '-DDMU + p, $p$ '-DDE concentration profiles for reference, it can be seen that the box cores collected at the USGS stations in 1992 are very similar to those obtained by the LACSD for nearby stations in 1991. The principle difference is that the depth at which maximum $p, p^{\prime}-\mathrm{DDE}+p, p^{\prime}$-DDMU concentrations occur are greater in the USGS cores. As discussed in Eganhouse and Pontolillo (2000), this is probably attributable to two factors: 1) loss of surficial sediments by the gravity corer relative to the box corer (Lee and others, 2002), and 2) differences in the locations of the USGS and LACSD stations (USGS stations were in shallower water closer to shore). Notwithstanding these effects, the patterns are quite similar. Relative abundances of $p, p$ '-DDMU in the 1991 LACSD cores increase slightly and become more variable with depth ( $p, p$ '-DDMU falling between 15 and 25 percent).

The amount of variability attributable to small scale spatial heterogeneity can be assessed by examining data for triplicate cores collected at LACSD stations 3C, 5C and 6C in 2005 (fig. 2, 16, and 3 , respectively). In general, the shapes of the $p, p^{\prime}-\mathrm{DDE}+p, p^{\prime}$-DDMU concentration profiles and the depths at which maximum concentrations occur are reasonably consistent among replicates for a given station. The downcore trends in percent $p, p$ '-DDMU within the most heavily contaminated zone (approximately 0 to $40 \mathrm{~g} / \mathrm{cm}^{2}$ ) are also fairly consistent among replicates for all stations. However, at increasing depth (greater than $40 \mathrm{~g} / \mathrm{cm}^{2}$ ), where concentrations of $p, p$ '-DDE and $p, p$ '-DDMU are low, large variations in percent $p, p$ '-DDMU are seen, and these variations differ among the replicates. This variability is, again, probably mainly due to increased uncertainty in the measurements at these low concentrations. A list of whole-core inventories of $p, p$ '-DDE and $p, p^{\prime}$-DDMU for the $3 \mathrm{C}, 5 \mathrm{C}$, and $6 \mathrm{C}$ triplicate cores is shown in table 5. The relative standard deviations of whole-core inventories $(p, p$ $\mathrm{DDE}, p, p^{\prime}-\mathrm{DDMU}$, and the sum, $p, p$-DDE + $p, p^{\prime}$-DDMU) for replicate cores at all three stations range from 8 to 25 percent.

Comparing the profile for the LACSD 3C core collected in 1991 with those found in the 2005 cores (fig. 2), an increase in the abundance of $p, p$ '-DDMU with time can be seen. While abundances are higher in the 2005 cores at all depths for all replicates, the greatest increases are seen in the lower portions of the cores, particularly at the lower shoulder of the $p, p$-DDE $+p, p$ '-DDMU concentration peak. This is reflected in an increase in $p, p$ '-DDMU abundance from 20 to 40 percent (based on whole-core inventories; see table 5). In the case of the 1991 and 2005 cores from station 6C (fig. 3), the change in $p, p$ '-DDMU abundance is not as apparent, particularly in the upper portions of the cores; $p, p$ '-DDMU increased from about 14 to 20 percent during this period (based on whole-core inventories; see table 5). Because analyses of these cores were performed in the same laboratory by 
the same chemists using exactly the same methods, these differences are presumed to be real. This assumes that no alterations occurred during the 14 years that the 1991 cores were stored frozen prior to (re)analysis. This assumption is supported by the strong similarity between percent $p, p$ '-DDMU profiles shown for the 1992 124B1-DDT core collected and analyzed by the USGS/Reston lab (fig. 15A) and the $19913 \mathrm{C} 2$ core collected and analyzed by the LACSD (fig. 2A).

Examples of data obtained using the LACSD gravity corer (Bascom and others, 1982) and a hydraulically-damped piston corer within cell SU (see fig. 5C-5D for station locations) as part of the sediment displacement study (Science Applications International Corporation, 2005) are shown in figures 17 and 18. These samples were collected near LACSD station 7C, which is closer to the outfall system than station 6C (fig. 5B). With the exception of the first replicate piston core at station A (fig. $17 \mathrm{~B})$, results for which were highly anomalous, variability in the percent $p, p^{\prime}$-DDMU and $p, p$ '-DDE + $p, p$ '-DDMU concentration profiles among replicates within the heavily contaminated sediment layers (approximately 0 to $40 \mathrm{~g} / \mathrm{cm}^{2}$ ) appears to be greater for both corers than that observed at the 3C, $5 \mathrm{C}$ and 6C stations in 2005 by the LACSD. In general, $p, p$ '-DDMU abundance is fairly constant (at about 20 percent; for example, core A-3) or increases gradually with depth. In this study, all chemical analyses were performed by a single laboratory (Woods Hole Group Environmental Laboratories, Raynham, MA) and the cores were collected on a single cruise, so the differences among replicate cores and between corers must be due to variations in analytical precision, navigation, spatial heterogeneity and corer performance. Some of this variability undoubtedly stems from spatial differences in the degree to which contaminated sediments (from pre-capping material at the site or subsequently transported and deposited material) were mixed into cap material.

To summarize, the relative abundance of $p, p$ '-DDMU generally increases with depth in surficial sediments on the Palos Verdes Shelf. Variability in the inventories and the profiles for any given station depends on analytical precision, navigation, corer deployment and spatial heterogeneity of the seafloor. Between the early 1990s and approximately 2003 there has been a substantial increase (about 200 percent) in the relative abundance of $p, p$ '-DDMU at LACSD station 3C, whereas the increase at LACSD station 6C was modest (about 33 percent) but is considered real.

Information on the magnitude of change in $p, p$ '-DDMU abundance over time is limited to comparisons of the LACSD 1991 and 2005 cores at stations 3C and 6C and the 1992 and 2003 cores collected by the USGS near station 3C (discussed below). For purposes of examining the spatial variation in $p, p$ '-DDMU abundance across the shelf, the best available data come from the analysis of USGS cores collected in 1992. $P, p$ '-DDMU abundances within the upper $4 \mathrm{~cm}$ (panel A), the upper 8 $\mathrm{cm}$ (panel B), and over the corer penetration depth ('whole-core'; panel C) are shown in figure 19. Whole-core data are not presented for all stations because only a small number of the box cores penetrated deeply enough to capture the complete history of effluent sedimentation. In general, it appears that in the early 1990s within the uppermost layers of sediment on the shelf (as represented by the upper $4 \mathrm{~cm}$ ) higher abundances of $p, p$ '-DDMU were present along the shallow inshore areas (between 30 and $60 \mathrm{~m}$ water depths). $P, p$ '-DDMU abundance generally decreased cross-shelf in the direction of the slope and basin. If a deeper section is considered $(8 \mathrm{~cm})$, the pattern changes such that the nearshore and offshore stations show lower abundances; highest percentages are found near to or along the 60-meter contour. The limited data available for whole-core inventories, all along the 60meter isobath, show a very gradual trend of increasing $p, p$ '-DDMU abundances with distance from the outfall system. However, the range is small (15 to 20 percent), indicating that in 1992 there was little difference in the fraction of $p, p$ '-DDE that had potentially been reductively dechlorinated along the 60meter isobath. The whole-core pattern is similar to data for cores collected in 1991 at LACSD stations 3C and 6C (fig. 20A-20B), which yielded p,p'-DDMU abundances of 20 and 14 percent, respectively. 
By 2005, p,p'-DDMU abundance had increased (39 to 43 percent: $\mathbf{3 C}, 16$ to 23 percent: 5C, 18 to 21 percent: 6C). These spatial patterns are qualitatively similar to the results of microcosm studies conducted by Quensen and others $(1998,2001)$ and Deming and Carpenter (2000) which showed higher $p, p$ '-DDE dechlorination rates with distance from the outfall system. For comparison, there were 11 cores collected as part of the sediment displacement study for which bulk densities were measured and inventories could be calculated (namely, K4-1, K3-1, K2-1, K-1, B-1, F-1, N-1, N1-1, N2-1, N3-1, N4-1; number before hyphen is the station name, number after hyphen indicates replicate number; see fig. 5C-5D for station locations). The $p, p$ '-DDMU abundances in these cores based on whole-core inventories ranged from 15 to 20 percent $(17.6 \pm 1.4$ percent; mean \pm 1 s.d.; data not shown), consistent with the data shown for LACSD station 6C in figure 20C.

\section{USGS core comparison}

The following discussion presents a comparison of geochemical data for cores collected by the USGS in 1992 and 2003 at station 522. Data on the vertical distribution of bulk properties (density, TOC, TN, TEO) and trace contaminants (DDTs, PCBs, LCABs) in the cores will be discussed. The latter are used to develop estimates of first-order transformation rates of $p, p$ '-DDE to $p, p$ '-DDMU during the period 1992-2003. These rates are then compared with rates estimated from whole-core inventories of $p, p$ '-DDE at station 522 and rates computed from the change in inventory of $p, p$ '-DDE over time at station 3C using LACSD monitoring data (C.R. Sherwood and B. Ferré, written commun., 2007). Next, a brief discussion of degradation pathway(s), possible products and environmental factors controlling DDE degradation rates is offered. Finally, a model of the three-step transformation pathway leading from $p, p$ '-DDE to $p, p^{\prime}$-DDMU to $p, p^{\prime}$-DDNU (reductive dechlorination) to products unknown is presented.

\section{Bulk properties}

Eganhouse and Pontolillo (2000) presented data demonstrating that at LACSD station 3C (1981-1993) and station 522 (1992), density profiles reflect the effects of burial, compaction and mixing over time. Vertical profiles of density at stations along the $60-\mathrm{m}$ contour typically consist of three layers: an upper interval of relatively higher densities ( 0 to $15 \mathrm{~cm}$ ), an interval with relatively low densities $(15$ to $30 \mathrm{~cm}$ ), and a deeper interval (greater than $30 \mathrm{~cm}$ ) with densities approaching those of native sediments. It is well known that this three-layer system is a direct reflection of the burial of relatively organic-rich, low density particulate matter derived from wastewater effluent during the period of peak waste emissions and its burial by predominantly natural sediments subsequent to the reduction of waste solids emissions starting in the early 1970s (Lee, 1994a; Lee and others, 2002).

Vertical profiles of bulk density for the cores collected by the USGS in 1992 and 2003 at station 522 are shown in figure 21A. The two cores have remarkably similar profiles showing the characteristic three-layer system with the low density effluent-affected horizon from about 15 to $35 \mathrm{~cm}$ (1992 core) and 20 to $40 \mathrm{~cm}$ (2003 core) in the sediment column. Qualitatively, features in the profiles appear to be vertically offset from each other. However, at all depths for which features in these profiles correspond to each other, the 2003 core has higher densities than the 1992 core. Together these observations suggest that since 1992 sediments at this location have undergone changes due to several processes. First, continued burial with input of increasingly coarser sediment has caused the density profile to shift downward resulting in higher densities in near-surface layers (less than $5 \mathrm{~cm}$ ) of the 2003 core than in the 1992 core. Sediments deposited since 1992 would likely be denser than sediments deposited before 1992 if relative inputs from natural sources such as the Portuguese Bend 
Landslide had increased (PBL; Eganhouse and Pontolillo, 2000; Kayen and others, 2002). Second, the effect of compaction is seen in the steeply increasing densities in the upper $4 \mathrm{~cm}$ of both profiles and generally higher densities in the 2003 core at time-equivalent (coeval) depths of deeper sections (greater than $28 \mathrm{~cm}$ for 1992, greater than $36 \mathrm{~cm}$ for 2003). Third, mixing could cause erosion of the upper shoulder of the low density effluent-affected layer (less than $26 \mathrm{~cm}-1992)$. The result would be an increase in density of the upper shoulder of the 2003 core (from both compaction and mixing with coarser sediments). However, based on comparison of the shapes of the profiles, there appears to be little or no evidence for erosion of the upper shoulder of the low density effluent-affected layer. This suggests that mixing of surficial sediments was likely confined to relatively shallow depths and did not reach the heavily contaminated effluent-affected sediment layers. This is consistent with evidence from ${ }^{234} \mathrm{Th}$ profiles and the abundance of benthic infauna in these sediments (Wheatcroft and Martin, 1994; Stull and others, 1996; Santschi and others, 2001). As shown in figure 13B, this is also consistent with data on the vertical distribution of conservative trace organic contaminants like the PCBs. The progressive burial, compaction, and coarsening of sediment inputs at or near station $3 \mathrm{C}$ is illustrated further in figure 21B where dry densities for the 1992 and 2003 USGS cores as well as cores from LACSD station 3C for the period, 1981 to 1993 (four year intervals), are plotted.

Data for the ratio of two pentacyclic triterpanes, $17 \alpha(\mathrm{H}), 18 \alpha(\mathrm{H}), 21 \beta(\mathrm{H})-28,30$-bisnorhopane $\left({ }^{*} \mathrm{C} 28\right)$ and $17 \alpha(\mathrm{H}), 21 \beta(\mathrm{H})$-hopane ('hopane'), in sediment cores at or near station 3C illustrate the potential significance of changing sediment inputs over time (figure 22). The pentacyclic triterpanes are a class of hydrocarbons found ubiquitously in fossil organic matter where hopane, a $\mathrm{C}_{30}$ compound, is often the most abundant member (Peters and others, 2005). The bisnorhopane ( $\left.{ }^{*} \mathrm{C} 28\right)$, a $\mathrm{C}_{28}$ compound of the same class, has somewhat restricted occurrence in crude oils but is very abundant in oils produced from the Monterey Shale. Consequently, this triterpenoid has been found pervasively in sediments and in oil seeps of the southern California Bight where it is regarded as an indicator of natural oil seepage or shale erosion (see references in Eganhouse and Venkatesan, 1993). The bisnorhopane is absent or present in exceedingly low concentrations in the JWPCP's wastewater effluent and in urban runoff from the Los Angeles River because non-California sources of refined petroleum products dominate (Eganhouse and others, 1981: Eganhouse and Kaplan, 1982). Consequently, the ${ }^{*} \mathrm{C} 28$ /hopane ratio can be used to differentiate waste-derived hydrocarbons from indigenous natural sources (Eganhouse and Kaplan, 1988). Data for a core collected by the LACSD at station 3C in 1981 (designated 3C1; see table 2) is shown in figure 22A. In the 3C1 core, the ${ }^{*} \mathrm{C} 28$ /hopane ratio remains around 0.2 through the upper $10 \mathrm{~g} / \mathrm{cm}^{2}$ of the core. Below a depth of 10 $\mathrm{g} / \mathrm{cm}^{2}$, the ratio begins to increase irregularly until a dramatic shift occurs at the bottom of the core. Based on the distribution of several molecular markers (LCABs, DDT), TOC, TN and stable isotopic composition $\left(\delta^{13} \mathrm{C}, \delta^{14} \mathrm{~N}\right)$ of the organic matter, Eganhouse and Kaplan (1988) showed that the depth at which the ${ }^{*} \mathrm{C} 28 /$ hopane ratio reaches a maximum of 2.5 marks the pre-effluent horizon in the $3 \mathrm{C} 1$ core.

${ }^{*} \mathrm{C} 28$ /hopane ratio profiles for the $124 \mathrm{~B} 1$ cores are shown in figure 22B. Data are plotted with an offset of $4 \mathrm{~g} / \mathrm{cm}^{2}$ for the 1992 core as described earlier (see Core Alignment). Minimum ratios in the two cores were $0.20(124 \mathrm{~B} 1-\mathrm{DDT})$ and $0.25(124 \mathrm{~B} 1-\mathrm{CH})$, similar to those typical of the highly contaminated upper $10-\mathrm{g} / \mathrm{cm}^{2}$ portion of the $3 \mathrm{C} 1$ core. Because the $124 \mathrm{~B} 1$ cores were collected at somewhat shallower water depths than and inshore of station $3 \mathrm{C}$, one might expect the ratios in the most contaminated layers to be slightly higher than 0.2 because inputs of Monterey Shale erosion debris derived from the PBL would be greater (Eganhouse and Pontolillo, 2000). At depths greater than about $30 \mathrm{~g} / \mathrm{cm}^{2}$ in the 124B1 cores, the ratios increase irregularly and are roughly equivalent (about 0.3 to 0.4 ). This portion of the profiles corresponds approximately to the 10 to $28 \mathrm{~g} / \mathrm{cm}^{2}$ depth 
interval in the $3 \mathrm{C} 1$ core (fig. 22A) where the relative contributions of effluent and natural sediments are known to have varied (1950 to 1971; Eganhouse and Pontolillo, 2000). However, it is apparent from comparison of figures $22 \mathrm{~A}$ and $22 \mathrm{~B}$ that the box corer used to collect the 124B1 cores did not penetrate into the pre-effluent layer because the ratio never exceeds 0.4 . The fact that the 2003 core has slightly higher ratios than the 1992 core at most depths probably indicates that there has been a small, but consistent, long-term difference in relative inputs of natural and effluent particles at the locations where these two cores were collected (fig. 6). At shallower depths in the 124B1 cores (less than about $15 \mathrm{~g} / \mathrm{cm}^{2}$ ), the ratios appear to diverge, with the 2003 core showing increasingly higher values than the 1992 core as the sediment-water interface is approached. This pattern may indicate that input of natural sediments derived from sources such as the PBL (Kayen and others, 2002) compared to effluent particles has increased at this site since at least 1992. These interpretations are consistent with the features of the bulk density profiles shown in figure $21 \mathrm{~A}$.

Aligned vertical concentration profiles for total organic carbon (TOC) and total nitrogen (TN) in the 1992 and 2003 cores are shown in figure 23. Although less distinct for TOC and TN than bulk density, the correspondence between the core profiles is evident. Whole-core inventories for bulk properties (TOC, TN, TEO) and trace constituents (DDTs, PCBs, LCABs) in the 124B1 cores along with whole-core inventories for $p, p$ '-DDE and $p, p^{\prime}$-DDMU in the 1991 and 2005 cores collected by the LACSD at stations $3 \mathrm{C}$ and $6 \mathrm{C}$ are given in table 5. The inventory of TOC in the 2003 core is 9.6 percent less than for the 1992 core and the inventory of TN is 23.2 percent lower. The difference in TOC inventories exceeds the analytical uncertainty (approximately 1.5 percent) and is considered to partly be the result of loss during diagenesis. The greater apparent decrease in TN relative to TOC is consistent with preferential degradation of nitrogen-bearing organic substances, such as proteins, during early diagenesis (for example, Martens and others, 1992; Macko and others, 1993). Some of the difference in TOC inventories may be attributable to the fact that the 2003 core did not penetrate as deeply into the sediment column as did the 1992 core. Thus, the 2003 core probably captured slightly less of the effluent-affected sediments than did the 1992 core. Additional inputs of organic carbon since 1992 (in surficial layers of the 2003 core) apparently were not sufficient to compensate for diagenetic losses and/or incomplete recovery of the effluent-affected sediment.

Aligned vertical concentration profiles of the total extractable organic matter (TEO) in the 1992 and 2003 cores are illustrated in figure 24. The solvents used for extraction of these sediments ranged from polar (methanol) to relatively non-polar (dichloromethane). Consequently, the total extractable organic matter is composed of a very complex mixture of substances that vary in structure, physico-chemical properties and susceptibility to biodegradation. Included in this mixture are hydrocarbons (themselves highly complex mixtures; Eganhouse and Kaplan, 1988), fatty acids, fatty alcohols, terpenoids, steroids, pigments, waxes and other substances bearing multiple functionalities. Subsurface maxima in the TEO profiles for the 1992 and 2003 cores are present at roughly the same depth (before alignment), and the whole-core inventory of TEO in the 2003 core is 38.7 percent lower than in the 1992 core. This strongly suggests that degradation of organic matter, evidenced by a decline in TOC and TN inventories, is even more extensive for the mixture of organic compounds extracted with organic solvents (see references in Hedges and Prahl, 1993). Alternatively, labile portions of the TEO may undergo reactions that result in their incorporation into organic macromolecules that are insoluble in organic solvents. In a sediment core collected near station $6 \mathrm{C}$ in 1992, a systematic decline in the relative abundance of oxygen-bearing fractions, such as the fatty acids, and other polar compounds with increasing sub-bottom depth (Eganhouse, unpublished data) has been observed. This supports the idea that components of the solvent extractable organic matter are preferentially removed during early diagenesis. 
Table 5. Whole-core inventories of bulk constituents and trace contaminants in cores collected by the USGS in 1992 and 2003 and by the Los Angeles County Sanitation Districts in 1991 and 2005.

$\left[\mathrm{mg} / \mathrm{cm}^{2}\right.$, milligrams per square centimeter; $\mu \mathrm{g} / \mathrm{cm}^{2}$, micrograms per square centimeter; $\mathrm{ng} / \mathrm{cm}^{2}$, nanograms per square centimeter; rep, replicate number]

\begin{tabular}{|c|c|c|c|c|c|c|c|c|c|c|c|c|c|}
\hline & TOC & TN & TEO & $p, p^{\prime}-\mathrm{DDE}$ & $p, p^{\prime}$-DDMU & $p, p^{\prime}$-DDNU & $\Sigma P C_{65}$ & $\Sigma \mathrm{PCB}_{18}$ & PCB 153 & $\Sigma \mathrm{LAB}_{26}$ & 6-C $\mathrm{C}_{12}$ & $\Sigma \mathrm{TAB}_{12}$ & TAB3 \\
\hline \multicolumn{14}{|c|}{ USGS } \\
\hline Station \#: & 522 & & & & & & & & & & & & \\
\hline 1992 & 762.2 & 54.36 & 223.91 & 216.6 & 64.96 & 13.60 & 25,806 & 8,891 & 1,075 & 45,036 & 5,454 & 17,288 & 3,821 \\
\hline 2003 & 688.7 & 41.72 & 137.2 & 123.0 & 86.70 & 18.07 & 28,932 & 9,257 & 1,131 & 20,805 & 1,996 & 15,773 & 3,872 \\
\hline $\mathrm{RPD}^{\mathrm{b}}$ (percent) & -9.6 & -23.2 & -38.7 & -43.2 & 33.5 & 32.9 & 12.1 & 4.1 & 5.3 & -53.8 & -63.4 & -8.8 & 1.3 \\
\hline \multicolumn{14}{|c|}{ LACSD } \\
\hline Station \#: & $3 \mathrm{C}$ & & & & & & & & & & & & \\
\hline 1991 & rep 2 & & & 307.1 & 78.3 & $--^{b}$ & -- & -- & -- & -- & -- & -- & -- \\
\hline \multirow[t]{3}{*}{2005} & rep 1 & & & 119.3 & 76.5 & -- & -- & -- & -- & -- & -- & -- & -- \\
\hline & rep 2 & & & 162.0 & 120.4 & -- & -- & -- & -- & -- & -- & -- & -- \\
\hline & rep 3 & & & 181.7 & 123.4 & -- & -- & -- & -- & -- & -- & -- & -- \\
\hline Mean $_{2005}$ & & & & 154.4 & 106.8 & -- & -- & -- & -- & -- & -- & -- & -- \\
\hline Std. dev.2005 & & & & 31.9 & 26.2 & -- & -- & -- & -- & -- & -- & -- & -- \\
\hline $\mathrm{CV}_{2005}$ (percent) & & & & 20.7 & 24.6 & -- & -- & -- & -- & -- & -- & -- & -- \\
\hline \multirow[t]{4}{*}{ RPD'91-05 (percent) } & rep 2-rep 1 & & & -61.1 & -2.2 & -- & -- & -- & -- & -- & -- & -- & -- \\
\hline & rep 2-rep 2 & & & -47.2 & 53.8 & -- & -- & -- & -- & -- & -- & -- & -- \\
\hline & rep 2-rep 3 & & & -40.8 & 57.7 & -- & -- & -- & -- & -- & -- & -- & -- \\
\hline & Mean & & & -49.7 & 36.4 & -- & -- & -- & -- & -- & -- & -- & -- \\
\hline Station \#: & $6 \mathrm{C}$ & & & & & & & & & & & & \\
\hline 1991 & rep 1 & & & 1279.1 & 206.7 & -- & -- & -- & -- & -- & -- & -- & -- \\
\hline \multirow[t]{4}{*}{2005} & rep 1 & & & 1048.9 & 235.6 & -- & -- & -- & -- & -- & -- & -- & -- \\
\hline & TOC & TN & TEO & $p, p^{\prime}-\mathrm{DDE}$ & $p, p^{\prime}$-DDMU & $p, p^{\prime}$-DDNU & $\Sigma \mathbf{P C B}_{65}$ & $\Sigma \mathrm{PCB}_{18}$ & PCB 153 & $\Sigma \mathbf{L A B}_{26}$ & 6-C $C_{12}$ & $\Sigma \mathrm{TAB}_{12}$ & TAB3 \\
\hline & rep 2 & & & 939.8 & 244.4 & -- & -- & -- & -- & -- & -- & -- & -- \\
\hline & rep 3 & & & 1103.5 & 280.6 & -- & -- & -- & -- & -- & -- & -- & -- \\
\hline Mean $_{2005}$ & & & & 1030.8 & 253.5 & -- & -- & -- & -- & -- & -- & -- & -- \\
\hline Std. dev.2005 & & & & 83.3 & 23.9 & -- & -- & -- & -- & -- & -- & -- & -- \\
\hline $\mathrm{CV}_{2005}$ (percent) & & & & 8.1 & 9.4 & -- & -- & -- & -- & -- & -- & -- & -- \\
\hline \multirow[t]{4}{*}{ RPD'91-'05 (percent) } & rep 1-rep 1 & & & -18.0 & 14.0 & -- & -- & -- & -- & -- & -- & -- & -- \\
\hline & rep 1-rep 2 & & & -26.5 & 18.2 & -- & -- & -- & -- & -- & -- & -- & -- \\
\hline & rep 1-rep 3 & & & -13.7 & 35.7 & -- & -- & -- & -- & -- & -- & -- & -- \\
\hline & Mean & & & -19.4 & 22.6 & -- & -- & -- & -- & -- & -- & -- & -- \\
\hline
\end{tabular}

${ }^{\mathrm{a}} \mathrm{RPD}=$ relative percent difference.

${ }^{b}$ Data not available. 


\section{Trace contaminants}

\section{DDTs}

Montrose Chemical Corporation released DDT-bearing caustic process liquors to the sewer system serviced by the LACSD from 1953 to 1971. Based on 18 samples collected in 1970 to 1971, the DDT composition of the Montrose effluent appears to have been dominated by the $p, p^{\prime}-$ and $o, p$ '-DDT (approximately 75 percent) with lesser amounts of DDEs (approximately 20 percent) and DDDs (approximately 5 percent; Redner and Payne, 1971; see 'Montrose 1970 and 1971', fig. 25A). Technical DDT contains little or no DDD and no DDE (Haller and others, 1945). Thus, the relatively large amounts of DDDs and DDEs in Montrose's effluent must have been produced during handling of the waste prior to its discharge into the sewer. After Montrose's inputs to the sewer had largely been terminated (April 1970), samples taken downstream from the Montrose plant showed a different composition (DDTs/DDEs/DDDs = 14:38:48; 'sewer samples' in fig. 24A). The low abundance of DDTs and the high abundance of DDDs and DDEs led Carry and Redner (1970) to conclude that these materials had come from historical deposits in the sewer line where the composition had been altered by reductive dechlorination of an appreciable portion of the parent DDT to form DDD. The average composition of DDTs in the LACSD effluent from 1971 to 1990, a period when no Montrose wastes were entering the sewer system, is virtually identical to the 'sewer samples' collected downstream of the Montrose plant (see 'LACSD, 1971-1990', fig. 25A). This is because residual deposits in the sewer system were still entering the LACSD's treatment plant. No information is available on the composition of Montrose's wastes prior to 1970. However, it is likely that the DDT composition of wastes entering the LACSD treatment plant before 1970 was similar to that of the 18 samples of Montrose effluent collected in 1970 to 1971 (see discussion in Eganhouse and others, 2000).

Because waste treatment during the period of Montrose's DDT inputs (prior to June 1971) to the LACSD was mainly primary, it seems probable that the DDT composition of the wastewater effluent discharged from the outfall system to the Palos Verdes Shelf during this time was similar to the Montrose effluent as shown in figure 25A. However, sediment cores collected by McDermott and others (1974) at station 3C in 1972, only one year after cessation of DDT inputs by Montrose, showed a composition with very little DDT (approximately 6 percent), slightly more DDD (approximately 10 percent), and the remainder as DDE (approximately 84 percent). This led Eganhouse and others (2000) to conclude that the vast majority of the parent DDT had been dehydrochlorinated (to DDE) in the early stages of diagenesis. Subsequent sediment surveys by the LACSD (1981) and the USGS (1992) at and near station 3C showed similar compositions to those found in 1972, albeit with steadily declining abundances of the remaining parent DDTs (fig. 25A).

The average DDT compositions in the 124B1 cores collected and analyzed by the USGS in 1992 and 2003 are compared in figure 25B. Included in the data set are results for $p, p$ '-DDNU. This diagram shows that on average in 1992, $p, p$ '-DDT and $o, p$ '-DDT were near or below detection limits, DDDs made up 7.2 percent of total DDT $\left(\Sigma \mathrm{DDT}_{\mathrm{n}=8}\right)$, DDEs were 71 percent ( 8 percent $o, p^{\prime}-$ DDE, 63 percent $p$, $p^{\prime}$-DDE), $p$, $p$ '-DDMU was 17 percent, and $p$, $p$ '-DDNU was 4 percent. By 2003, the average composition had changed as follows: DDTs (near or below detection), DDDs-6.8 percent, DDEs-56 percent ( 8 percent $o, p$ '-DDE, 48 percent $p, p$ '-DDE), $p, p$-DDMU-30 percent, $p, p$-DDNU-6.1 percent. The main differences between the DDT compositions of these cores are in the relative abundances of $p, p^{\prime}-\mathrm{DDE}, p, p$-DDMU and $p, p^{\prime}$-DDNU, with $p, p$ '-DDE declining and $p, p$ '-DDMU and $p, p^{\prime}$-DDNU increasing. These changes suggest that transformation of $p, p$ '-DDE 
to $p, p^{\prime}$-DDMU and $p, p^{\prime}$-DDMU to $p, p^{\prime}$-DDNU was occurring during the 1992 to 2003 period. Concentration profiles of these three DDT compounds in the 1992 and 2003 cores are shown in figure 26. Here the depth axis is given in units of $\mathrm{g} / \mathrm{cm}^{2}$ to compensate for the effects of compaction. The increase in abundance of $p, p^{\prime}$-DDMU and $p, p^{\prime}$-DDNU relative to $p, p^{\prime}$-DDE at all depths is readily apparent.

Whole-core inventories in these cores can be compared to assess the magnitude of the changes in masses of $p, p^{\prime}$-DDE, $p, p$-DDMU and $p, p^{\prime}$-DDNU between 1992 and 2003. The assumption for such a comparison is that observed changes between the cores reflect in situ transformation, with no net exchange between the sediments and ocean waters. Evidence to support this assumption will be presented in following sections (Polychlorinated biphenyls and Long-chain alkylbenzenes). From 1992 to 2003, whole-core inventories of $p$, $p$ '-DDE decreased by 43.2 percent, whereas inventories of $p, p^{\prime}$-DDMU and $p, p^{\prime}$-DDNU increased by 33.5 percent and 32.9 percent, respectively (table 5). These differences exceed the estimated precision of the analytical method (15 to 20 percent). For comparison, table 5 also presents whole-core inventories of $p, p^{\prime}$-DDE and $p, p^{\prime}$-DDMU in cores collected by the LACSD in 1991 and 2005 at station 3C. The decrease in inventory for $p, p^{\prime}$-DDE ranges from 41 to 61 percent (mean difference $=50$ percent). Over the same period, the inventory of $p, p^{\prime}$-DDMU shows little change for one replicate and increases by 54 to 56 percent for the other two replicates (mean difference $=36.4$ percent). Taking into account the fact that the period between collection of the LACSD cores was approximately 3 years more than for the USGS cores, these results seem reasonably comparable. In a later section (Transformation pathway and products) the mass balance for DDE/DDMU/DDNU between the 1992 and 2003 USGS cores is examined in more detail.

\section{Polychlorinated biphenyls}

Attempts were made to quantify 84 PCB congeners, but due to interferences with major DDT compounds and other unidentified halocarbons in Palos Verdes Shelf sediments, concentrations of only $68 \mathrm{PCBs}$ could be determined with confidence (table $4 ; \Sigma \mathrm{PCB}_{65}$ ). The composition of the PCBs by chlorination level (also referred to as homolog distributions) in Palos Verdes Shelf sediments is shown in figure 27. Data used for construction of these bar diagrams include only the 68 PCB congeners mentioned above. As indicated by the small error bars, compositions were very uniform throughout both cores, and the homolog distributions were virtually identical in 1992 and 2003. Homolog distributions are also shown for representative Aroclor mixtures based on data of Frame and others (1996). In this case, data are for the same 68 congeners, with chlorination level percentages normalized to the sum of the abundances of those congeners. It must be noted that the methods used for quantitation of the Aroclor mixtures as reported by Frame and others (1996) and those used for analysis of the sediment samples shown in this figure differ considerably. Moreover, data for the Aroclors published in Eganhouse and others (2000) were based on studies by Schulz and others (1989), and the data for core 124B1-DDT shown in that publication included more of the targeted PCB congeners than have been included here. Consequently, distributions shown for 124B1-DDT and Aroclors in figure 27 appear somewhat different from those presented in Eganhouse and others (2000). Nevertheless, the conclusions are essentially the same.

The PCBs in Palos Verdes sediments are dominated by tetra-, penta- and hexachlorobiphenyls with lesser amounts of homologs having fewer than four and more than six chlorines. The composition is not attributable to a simple mixture of Aroclors (Eganhouse and others, 2000), but the dominance of the tetrachlorobiphenyls is suggestive of the presence of 
Aroclors 1242 and 1254 (or possibly 1248). In addition, the abundance of higher chlorinated PCBs including the octachlorobiphenyls and specific congeners not found in Aroclors 1242 and 1254 (for example, PCBs 195, 196; data not shown), indicates that other Aroclor mixtures, such as 1260 and/or 1262 or 1268, are present. The observation of other congeners in the sediment samples, which are not among the 68 for which data are shown here (for example PCBs 208, 209), further supports the likelihood that higher chlorinated Aroclors like 1268 are present. For comparison, the LACSD reported observing PCB compositions consisting of 56 to 78 percent Aroclor 1242, with the remainder as 1254, in effluent monitored during the period 1971 to 1984 . Unfortunately, the analytical methods used by the LACSD at that time were not congener-specific. This limits the ability to characterize the composition of PCBs being discharged from the LACSD plant during that time.

Vertical concentration profiles of the PCBs exhibit the characteristic subsurface maximum attributed to burial of more heavily contaminated sediments deposited in the late 1960s and early 1970s (Eganhouse and others, 2000). Vertical concentration profiles for $\Sigma \mathrm{PCB}_{65}, \Sigma \mathrm{PCB}_{18}$ and a representative congener, PCB 153, in the 1992 and 2003 cores are shown in figure 28. For a given core, the three profiles are very similar, and profiles for all PCB metrics are similar between cores. The similarity between profiles for a single core reflects the general uniformity in composition of the PCBs and supports the use of any of the three metrics to track the vertical distribution of PCBs in the sediment column. The similarity of profiles between cores (for a given metric) indicates that there has been no major alteration in the distribution of the PCBs at this site due to mixing and/or degradation between 1992 and 2003. This is reasonable because by 1992, the most heavily contaminated sediments at this location were already buried to depths greater than $25 \mathrm{~cm}$, appreciably below the most intensively bioturbated surficial layers (Drake and others, 1994; Wheatcroft and Martin, 1994; Stull and others, 1996; Swift and others, 1996; Lee and others, 2002). Comparison of the 1992 and 2003 profiles (see also fig. 13) suggests that the major impact during the intervening period was burial. The similarity in maximum concentrations of the two cores is likely fortuitous because spatial variation in PCB concentrations due to heterogeneity is expected to be around 20 to 25 percent (table 5). However, it is noteworthy that PCB concentrations in surficial layers of the cores are very similar, whereas $p, p$ '-DDE concentrations are reduced in 2003 (fig. 26). Remarkably, the whole-core inventories of $\Sigma \mathrm{PCB}_{65}, \Sigma \mathrm{PCB}_{18}$ and PCB 153 in the 1992 and 2003 cores differ by only 12, 4 and 5 percent, respectively (table 5). On a molar basis, the results are similar. For example, for $\Sigma \mathrm{PCB}_{18}$ whole-core inventories differ by only 3.3 percent. For all intents and purposes, the inventories of the PCBs in the 1992 and 2003 cores are the same.

To investigate the hypothesis that the PCBs are being reductively dechlorinated, patterns in the numbers of total, ortho- and meta- + para-substituted chlorines per biphenyl molecule were examined. Previous field and laboratory studies have shown that microbially-mediated reductive dechlorination, operating through at least eight identified 'processes', causes a shift in the distribution of PCBs to lower chlorinated species having a greater proportion of congeners substituted at the ortho- position (Bedard and Quensen, 1995; Wiegel and Wu, 2000). This occurs because the microorganisms carrying out the dechlorination reactions generally prefer chlorines attached at the meta- and para- positions. Ortho-dechlorination has rarely been reported (for example, Kuipers and others, 1999; Palekar and others, 2003). For this reason, it is instructive to examine the vertical profiles of total, ortho- and meta- + para-substituted chlorines per biphenyl in these sediment cores. The computation of molar abundances of total, ortho-, meta-, and parasubstituted chlorines per biphenyl was carried out as described by Quensen and others (1990). In 
the case of coeluting congener pairs (for example, 31/28, 128/167, 171/156,157/201), equal amounts of the congeners were assumed to be present in each peak, and average numbers of chlorines at ortho-, meta-, and para- positions were computed. In terms of reductive dechlorination, there are two ways to evaluate these data. First, one can examine trends in the vertical distribution of chlorine substituent abundance in a given core (that is, evidence of diagenesis; Magar and others 2005a,b). This depends on the assumption that the composition of PCBs introduced to the sediments is known and/or did not vary over time. The second approach is to compare PCB compositions in cores collected at the same location but at different times (Pakdeesusuk and others 2005; Bzdusek and others, 2006). In the following paragraphs both approaches are evaluated.

Vertical profiles of the molar abundances of total, ortho- and meta- + para-substituted chlorines per biphenyl moiety in the $124 \mathrm{~B} 1$ cores (the 68 congeners included in $\Sigma \mathrm{PCB}_{65}$ ) are shown in figure 29. Total chlorine abundances vary within a small range $(4.6$ to $5.1 \mathrm{Cl} /$ biphenyl) and decrease irregularly with depth in both cores. Because reductive dechlorination favors removal of meta- and para-substituted chlorines, one would expect that meta- + para- chlorine abundances would also decline with depth. As shown in figure 29B, this pattern is observed, and the profiles for meta + para chlorine abundances (2.9 to $3.4 \mathrm{Cl} /$ biphenyl) closely mimic those for total chlorine. The abundance of ortho-chlorines in the 124B1 cores varies within a very narrow range (1.56 to $1.61 \mathrm{Cl} /$ biphenyl), but it also decreases with depth in both cores. If dechlorination of PCBs had occurred over the period represented by these cores (approximately 40 to 50 years), one would expect to see a decrease in total and meta- + para-chlorine abundances with depth and little or no change in ortho-chlorine abundance (Magar and others, 2005b). Qualitatively, the patterns shown in figure 29 are consistent with reductive dechlorination having taken place in both cores.

However, it is also possible that these trends reflect a gradual change in PCB composition, whereby increasing amounts of more heavily chlorinated products were introduced over time. Mixing of surficial sediments throughout this period would serve to smooth any rapid short-term compositional variations of the inputs. This concept is illustrated in figure $30 \mathrm{~A}$, which presents data for the 124B1 cores along with Aroclor compositional data from Frame and others (1996)

Based on the abundance of total chlorine/biphenyl (or chlorines at specific positions), the 124B1 cores are most similar to Aroclor 1254 (fig. 30A). However, it is apparent that even a minor progressive change in composition of PCB inputs could result in the trends seen in figure 29. The same data are presented in figure $30 \mathrm{~B}$ in the form of a scatter plot of meta- + para-chlorine versus ortho-chlorine abundances. If the original sources of PCBs to the Palos Verdes Shelf sediments had chlorine substituent compositions similar to the Aroclors shown here, preferential dechlorination at meta- and para-chlorine positions would result in compositions plotting below the respective Aroclor data points as indicated by the downward facing arrow (Brown and Wagner, 1990). In the case of mixed Aroclor inputs, dechlorinated PCB mixtures would plot below the relevant Aroclor mixing lines. Instead, the Palos Verdes Shelf core data fall within a very narrow range of values near to but slightly to the left of the data point for Aroclor 1254. It is clear that a change in Aroclor composition with greater contributions from more highly chlorinated mixtures (for example, Aroclors 1260 or 1262) would result in a larger increase in the meta- + para-chlorine substituent abundance than ortho-chlorine abundance (ratio about 2:1) just as seen in figure 29. Thus, the decreasing abundance of total chlorine with depth in the 124B1 cores is most likely the result of changes in the composition of PCB inputs over time, not reductive dechlorination.

It is worth noting that the relative percent differences between maximum and minimum chlorine abundances in the 124B1 cores were as follows: 1992 core-meta- (10.1 percent), para- 
(9.4 percent), ortho- (5.6 percent), total ( 7.0 percent); 2003 core-meta- (10.8 percent), para- (5.9 percent), ortho- (8.7 percent), total ( 7.7 percent). This is depicted in figure 31 along with plots of $\mathrm{Cl}$-substitution abundance and vertical concentration profiles for $\Sigma \mathrm{PCB} \mathrm{B}_{65}$. Whether the downcore variations seen in figure 29 are due to compositional changes of PCB inputs over time, reductive dechlorination or some combination of the two, the rates of change have been exceedingly slow. For example, linear regression analysis of a plot of meta- + para-chlorine abundance compared to estimated age of sediments in core 124B1-DDT (based on molecular stratigraphy) yields an 'apparent dechlorination rate' of 0.0078 moles $\mathrm{Cl} /$ biphenyl per year $\left(\mathrm{r}^{2}=0.79\right)$. By comparison, Magar and others (2005b) reported an average rate of 0.094 moles $\mathrm{Cl} /$ biphenyl per year for ten cores collected from Lake Hartwell, SC.

To further examine the possibility that PCBs in Palos Verdes Shelf sediments may have undergone reductive dechlorination, an analysis of the differences in molar PCB compositions in different sections of the 124B1 cores was conducted. The difference in molar abundance of the 65 PCB peaks for the deepest and uppermost sections of each of the two 124B1 cores is shown in figure 32. These bar diagrams clearly show that there are small but consistent differences in composition between the deepest and shallowest sections of these cores, with lower amounts of the more highly chlorinated PCBs at depth. Although the patterns for the two cores show similarities, they are not identical. Based on published information on the expected products of various dechlorination reaction processes (Bedard and Quensen, 1995), PCB congeners that appear to be enriched in the 124B1 core bottoms relative to the core tops (\#1, 8, 6, 17, 31/28, 25, 19, 26, 18, 47, $49,52,44,91)$ were examined. The assemblage of potential 'products' is most consistent with what has been termed the process H' (or possibly $\mathrm{H}$ ) dechlorination pattern. Process $\mathrm{H}^{\prime}$ is characterized by meta dechlorination of 23-, 234- and possibly 236-chlorophenyl groups and para dechlorination of 34- and 245-chlorophenyl groups. [Note: Chlorine atom substitution on PCB congeners is indicated as xxx-yyy, where $\mathrm{x}=$ positions on first phenyl group, and yyy $=$ positions on second phenyl group.] Based on this, specific congener pairs that might indicate operation of process H' within the cores were identified. Pairs of interest consist of: 1) a parent showing a decreased molar abundance in the core bottom, and 2) a daughter that shows an increased molar abundance in the core bottom. This is illustrated in figure 32 by colored dots used to identify the following major parent-daughter dechlorination pairs: $138 \rightarrow 87$ (234-245 $\rightarrow 234-25$; red dots), $105 \rightarrow 66$ (2ㄹㄴ-34 $\rightarrow 24-34$; blue dots), 153 $\rightarrow 101$ (245-245 $\rightarrow 245-25$; green dots, 2003 core only), and $118 \rightarrow 70(2 \underline{4} 5-34 \rightarrow 25-34$; black dots). Underlined numbers indicate substituents that would be removed during reductive dechlorination for a specified parent-daughter pair. Although these congener pairs are consistent with process $H^{\prime}$ dechlorination patterns published in the literature, a number of major apparent products were found for which no viable process $H^{\prime}$ parent could be identified. Similarly, losses of apparent parents could not be linked to the production of daughters. Given the magnitude of the core bottom versus core top differences, the limited number of potential parent/daughter pairs, and the obvious lack of mass balance between them, evidence for reductive dechlorination in Palos Verdes Shelf sediments does not appear compelling. Nevertheless, the possibility that some reductive dechlorination, limited in scope, may have occurred over the period encompassed by these cores cannot be entirely ruled out.

Although the mean difference in total chlorine abundance between the two cores is small (average RPD $=3.0 \pm 2.4$ percent; see fig. 29A), the generally higher abundances found for the 2003 core are inconsistent with reductive dechlorination during the period 1992 to 2003 . Meta- + para-chlorine abundances for the 2003 core are also, in most cases, greater than for the 1992 core, whereas ortho-chlorine abundances for the 2003 core are generally very close to or slightly higher 
than that for the 1992 core (fig. 29B-29C). The similarity in downcore trends for the two cores suggests that these small between-core differences in chlorine abundance may simply reflect analytical uncertainty or spatial variability in PCB compositions. Congener distributions in core sections of equivalent age offer one means of examining this issue in greater detail.

Finally, in terms of the period 1992 to 2003 it is instructive to compare PCB substitution patterns for the two cores at coeval depths. PCB compositions at the depth of maximum concentration ( 30 to $32 \mathrm{~cm}$ or $29.8 \mathrm{~g} / \mathrm{cm}^{2}-1992,34.1 \mathrm{~g} / \mathrm{cm}^{2}-2003$ ) are shown in figure 33 . Compositional differences are, again, generally quite small (panel C). If reductive dechlorination had taken place in these sediments between 1992 and 2003 one would expect to see increases in some lower chlorinated congeners (left side of diagram) with corresponding decreases in abundances of some higher chlorinated congeners (right side of diagram) and an increased proportion of ortho-chlorine substituted species. However, no such pattern is observed. In fact, for most congeners, the difference between the cores is exceedingly small and there is no systematic relationship between congeners recognizable as one of the established dechlorination processes. Taken together, the evidence strongly suggests that reductive dechlorination of the PCBs has not occurred to any significant extent on the Palos Verdes Shelf since at least 1992. This is consistent with the fact that whole-core inventories of $\Sigma \mathrm{PCB}_{65}, \Sigma \mathrm{PCB}_{18}$ and PCB 153 in the $124 \mathrm{~B} 1$ cores are virtually identical (table 5). Based on the distribution of chlorine substituents among congeners as compared with Aroclor compositions (fig. 30B), it also seems highly unlikely that reductive dechlorination occurred prior to 1992. The question that remains is, "Why"?

The type, extent, and rate of dechlorination depend on environmental factors that affect the growth and metabolic activities of dechlorinating microorganisms (Wiegel and $\mathrm{Wu}, 2000$ ). Through a variety of laboratory experiments, the importance of several of these factors has been demonstrated. However, their effects in controlling dechlorination are complex. Among the factors that are considered important are the following: temperature, $\mathrm{pH}$, availability of metabolizable organic matter, availability of hydrogen, availability of electron acceptors (including the PCBs themselves), bioavailability, and toxicity. One of the most consistent observations is that while organisms capable of dechlorinating PCBs has been reported in uncontaminated sediments, the rate and extent of dechlorination of added PCBs is much greater in contaminated sediments. It is possible that microorganisms capable of dechlorinating PCBs in Palos Verdes Shelf sediments are either not present, or if present are not active. Further research is needed to answer this question.

\section{Long-chain alkylbenzenes}

Two classes of long-chain alkylbenzenes were determined in this study, the linear alkylbenzenes (LABs) and the tetrapropylene-based alkylbenzenes (TABs). As discussed earlier, the LABs consist of 26 secondary phenylalkanes with linear alkyl chains, whereas the TABs are an exceedingly complex mixture of alkylated benzenes with branched alkyl chains (fig. 9). The TABs were sulfonated to produce the synthetic surfactants known as TBS (tetrapropylenebenzene sulfonates) which replaced soap in the early 1950s (Swisher, 1987). By the late 1950s to early 1960s it became apparent that components of TBS were highly resistant to microbial degradation, causing undesirable foaming in wastewater treatment plants and receiving waters. Consequently, a more degradable substitute having the desirable surface-active properties of TBS was sought. This led to development of the LABs, a class of long-chain alkylbenzenes having linear rather than branched alkyl chains, and the rapid replacement of the TBS by LAS (linear alkylbenzene sulfonates-formed by sulfonation of LABs) in the United States during the mid-1960s (fig. 34). 
The key point is that although the LABs and TABs have similarities in structure and, thus, physicochemical properties, the biodegradability of these two types of long-chain alkylbenzenes under aerobic conditions differs appreciably.

The LABs are a synthetic mixture, consisting of five homolog groups differing by chain length $\left(\mathrm{C}_{10-14}\right.$-benzenes) with all possible secondary phenylalkanes present within each homolog group (see fig. 9). Thus, their structures vary systematically. Changes in the composition of the LABs in environmental settings occur as a result of physical partitioning and biodegradation, and these changes can be used to infer the operation of natural processes (Eganhouse, 2004). When synthesized, the LABs have roughly equivalent abundances of isomers for a given homolog group (see fig. 9). Sulfonation of the LABs to form LAS is not complete, so a small residual amount, usually much less than 1 percent of LAS (Takada and Ishiwatari, 1987), is carried over into commercial detergents (Eganhouse and others, 1983a). The isomer distribution of the residual LABs is not altered during sulfonation, so the nearly equivalent abundances of LAB isomers are maintained when LABs are introduced to sewage treatment systems in association with disposal of detergent wastewater (or sometimes, as in the case of the JWPCP, industrial inputs). During waste treatment LABs are either removed or released as a component of the treated effluent.

When LABs enter the environment they can undergo microbial degradation. Under aerobic conditions, this has been shown to result in selective removal of the so-called 'external' isomers as compared with 'internal isomers' (Eganhouse and others, 1983b; Bayona and others, 1986; Takada and Ishiwatari, 1987; Takada and Ishiwatari, 1989). 'External' isomers have structures in which benzene is attached nearer the end of the alkyl chain, whereas 'internal' isomers have structures with benzene attached toward the middle of the chain. For example, among the phenyldodecanes, $3-\mathrm{C}_{12}$ and $2-\mathrm{C}_{12}$ are considered 'external' isomers and $6-\mathrm{C}_{12}$ and $5-\mathrm{C}_{12}$ are considered 'internal' isomers. To characterize the isomer distribution of LAB mixtures, Takada and Ishiwatari (1987) proposed an index, the I/E ratio, that can serve as an indicator of changes in isomer distribution caused by biodegradation. It is defined as:

$$
\mathrm{I} / \mathrm{E} \text { ratio }=\left[6-\mathrm{C}_{12}+5-\mathrm{C}_{12}\right] /\left[4-\mathrm{C}_{12}+3-\mathrm{C}_{12}+2-\mathrm{C}_{12}\right]
$$

There is at least one report of aerobic biodegradation of the LABs that does not entirely follow the isomer selective degradation pattern (Bhatia and Singh, 1996). Less is known about the degradation of LABs under anaerobic conditions. In short-term anoxic wastewater degradation experiments Takada and Ishiwatari (1989) reported no degradation of the LABs. Similar results were obtained by Steber and others (1995) in an investigation of the anaerobic biodegradability of LABs as potential drilling fluids. However, other studies (Raymundo and Preston, 1992; Lamoureux and others, 1996; Eganhouse and others, 2000; Johnson and others, 2001) have suggested that the LABs may be degradable under anaerobic conditions.

An examination is now made of LAB data obtained for the LACSD wastewater effluent and cores from the Palos Verdes Shelf (fig. 35). The average homolog (that is, chain length) distribution of LABs in LACSD effluent samples collected in 1979 (Eganhouse and others, 1983b) and 1990 (Hendricks and Eganhouse, 1992) as well as in sediment cores collected by the LACSD in 1981 at station 3C (Eganhouse and others, 1983b) and the two 124B1 cores collected in 1992 and 2003 is depicted in figure 35A. In general, the dominant chain lengths for effluent and sediment samples are $\mathrm{C}_{11}$ and $\mathrm{C}_{12}$. Between 1979 and 1990 the dominant chain length in the effluent appears to have shifted from $\mathrm{C}_{11}$ to $\mathrm{C}_{12}$. By comparison, sediment core data show a reduction in the proportion of shorter chain homologs and an increase in the proportion of longer 
chain homologs compared to the effluent. Similar patterns were noted by Takada and Ishiwatari (1987) for wastewater samples, detergents and suspended particles in Japanese rivers. Because the hydrophobicity of the LABs increases with chain length (Sherblom and others, 1992), this relationship probably reflects alteration of the homolog distribution of the LABs through dissolution (and possibly biodegradation) of the more soluble short-chain homologs (see references in Eganhouse and others, 2000). The cause for observed differences in the homolog distribution among the three cores is less obvious.

Vertical profiles of the I/E ratio in the three sediment cores along with data for the LACSD effluent samples are plotted in figure 35B. Effluent I/E ratios range from 0.59 to 0.85 (1979) and from 0.72 to 0.86 (1990). As expected, these ratios fall within the range reported for synthetic detergents (Takada and Ishiwatari, 1987; Eganhouse, unpublished data). By contrast, ratios in the sediment cores are all greater than 1, ranging from 1.24 to 2.3. The difference between effluent and sediment I/E ratios is probably due to aerobic biodegradation that occurred shortly after discharge of the treated wastewater effluent. The I/E ratio profile of the 124B1-DDT (1992) core shows some downcore variation with a general trend of slightly decreasing ratios with depth. The 124B1$\mathrm{CH}$ (2003) core I/E profile appears to also decrease slightly with depth. This suggests that if degradation of LABs occurs under anoxic conditions, there is no preference for external isomers as seen in oxic biodegradation experiments (Bayona and others, 1986; Takada and Ishiwatari, 1989). A comparison of whole-core inventories (table 5) during the 1992 to 2003 period, however, reveals that there has been a significant decline in the mass of LABs at this site; the changes in $\Sigma \mathrm{LAB}_{26}$ and 6- $\mathrm{C}_{12}$ inventories were -53.8 percent and -63.4 percent, respectively. Because there is no evidence for reductive dechlorination of the PCBs during this same period and because the PCB inventories have remained effectively constant, the decreases in LAB inventories are considered real. It would, therefore, appear that degradation of the LABs under anoxic conditions is taking place with little or no apparent isomer selectivity as seen under oxic conditions. This finding is consistent with data of Raymundo and Preston (1992) who reported little or no change in I/E ratio with depth in sediment cores from the Humber and Wash estuary despite declining concentrations of LABs (when normalized to organic carbon content).

As mentioned earlier, the TABs are considerably more complex than the LABs. In the original work of Eganhouse and others (1983b), the strong similarity between HRGC/MS chromatograms of a TAB mixture obtained from Monsanto Company (former manufacturer of TABs) and those in Palos Verdes Shelf sediments at station 3C suggested that these compounds were well preserved. Preservation in sediments, particularly under anoxic conditions, would be expected for the TABs given their branched alkyl chains. For purposes of quantitation, twelve major TAB peaks were selected, the most prominent of which in sediment samples is TAB peak \# 8 (also referred to as TAB3; see figure 9). In subsequent studies Eganhouse and others (2000) presented evidence that while the TABs are largely preserved, subtle changes in the relative abundances of some of the TAB peaks can be seen as one moves deeper in the sediment column. It was unclear whether these changes reflected historical variations in TAB composition over time or selective removal. To expand on this concept, figure 36 presents data on the composition of the TABs in the 124B1 cores. The upper panel (fig. 36A) is a plot of the average compositions (with error bars) for the two cores. Although similar, the average relative abundances of the TABs in the two cores exhibit distinct differences. Notably, there is a decrease in the average relative abundance of TABs \#10 and 11, with smaller decreases for TABs \#4, 7 and 12. As indicated by the error bars, some of these differences are likely not significant (for example, TABs 4, 7 and 12). One way of resolving this is to compare compositions for core sections that were deposited at 
approximately the same time. The lower panel (fig. 36B) shows a comparison between compositions for the two cores at the same sub-bottom depth (30 to $32 \mathrm{~cm}$ or $29.8 \mathrm{~g} / \mathrm{cm}^{2}-1992,34.1$ $\left.\mathrm{g} / \mathrm{cm}^{2}-2003\right)$. Although the patterns are similar to those seen for the whole-core averages, some of the relationships are actually reversed (for example, TABs \#4, 7). A most notable result is the fact that the relative abundance of TAB3 (that is, TAB peak \#8) appears to have remained essentially the same during this time period. From these data, it is clear that some of the TABs are slowly being altered under what are presumed to be reducing conditions in sediments at this location. This, in itself is somewhat surprising given their structures. However, based on a comparison of the $\Sigma \mathrm{TAB}_{12}$ and TAB3 whole-core inventories (table 5), the effects of this transformation are not quantitatively important; from 1992 to 2003 the change in $\Sigma \mathrm{TAB}_{12}$ and TAB3 inventories were -8.8 percent and 1.3 percent, respectively. These differences are within the estimated analytical error of about 15 percent. For all intents and purposes, inventories of TAB3 and the sum of the twelve TAB peaks have remained constant. Because of the demonstrated persistence of these compounds, and particularly TAB3, changes in abundance of the LABs, a related class of compounds with similar physico-chemical properties, are considered highly significant. Based on comparison of inventory ratios in a 1981 core from LACSD station 3C and the 124B1-DDT (1992) core, Eganhouse and others (2000) suggested that TAB3 is more persistent than $\Sigma \mathrm{TAB}_{12}, \Sigma \mathrm{LAB}_{26}$ and 6$\mathrm{C}_{12}$ and $p, p$-DDE. It appears that these early interpretations have proven correct.

Aligned vertical concentration profiles of $\Sigma \mathrm{LAB}_{26}, 6-\mathrm{C}_{12}, \Sigma \mathrm{TAB}_{12}$, and TAB3 in the $124 \mathrm{~B} 1$ cores $\left(4-\mathrm{g} / \mathrm{cm}^{2}\right.$ offset) are shown in figure 37 . The $\mathrm{LAB}, 6-\mathrm{C}_{12}$, is included in this comparison because it is the single most abundant congener and because under oxic conditions, it is regarded as the most persistent of the phenyldodecanes. The marked reduction in $\Sigma \mathrm{LAB}_{26}$ and $6-\mathrm{C}_{12}$ concentrations at all but one depth in the 2003 core compared with the 1992 core is shown in panels $\mathrm{A}$ and $\mathrm{B}$. By contrast, vertical concentration profiles for $\Sigma \mathrm{TAB}_{12}$ and $\mathrm{TAB} 3$ in the $124 \mathrm{~B} 1$ cores (panels C, D) appear very similar, with maximum concentrations and concentrations at the tops and bottoms of the cores matching quite well. Clearly, the LABs are being removed while the TABs persist during early diagenesis.

\section{Transformation rates, pathways, products and kinetics}

In this section first-order transformation rates of $p, p$ '-DDE and selected LABs are calculated by directly comparing whole-core inventories and concentrations/inventories at coeval depths in the 124B1 cores (core-to-core comparison).

\section{Whole-core inventories}

Transformation rates of $p, p$ '-DDE were computed from whole-core inventories for the USGS (1992/2003) and LACSD (1991/2005) cores (table 6). Also shown in this table are data generated by C.R. Sherwood and B. Ferré (written commun., 2007) using LACSD core data along with results of the core-to-core comparison and microcosm experiments. In the case of the 124B1 cores collected near station $3 \mathrm{C}$ (that is, at station 522), a $p, p^{\prime}$-DDE transformation rate of $0.051 \mathrm{yr}^{-1}$ was calculated based on whole-core inventories. This agrees well with the mean and median rates for all sections of the aligned cores $\left(0.052\right.$ and $0.045 \mathrm{yr}^{-1}$; table 6$)$. For the LACSD cores, the following rates were obtained: $3 \mathrm{C}-0.085,0.058$, and $0.047 \mathrm{yr}^{-1}\left(\right.$ mean $\left.\pm 1 \mathrm{sd}: 0.064 \pm 0.020 \mathrm{yr}^{-1}\right)$, $6 \mathrm{C}-0.018,0.028$, and $0.013 \mathrm{yr}^{-1}\left(\right.$ mean $\left.\pm 1 \mathrm{sd}: 0.020 \pm 0.007 \mathrm{yr}^{-1}\right)$. Although the variability in these latter estimates for a given station is between 30 and 40 percent, the higher transformation rates at station $3 \mathrm{C}$ are considered to be significant. The average rate at LACSD station $3 \mathrm{C}$ is higher than 
that for the USGS cores collected at station 522. Part of this difference can be explained by the fact that the LACSD cores span a longer period of time (1991-2005 compared to 1992-2003). Because rates appear to have increased at or near station $3 \mathrm{C}$ over the last decade, it is conceivable that the higher rates at LACSD station 3C are caused by the fact that the LACSD cores encompass more of the time during which these changes occurred. It is also possible that environmental conditions at the two locations are somewhat different, favoring faster rates of reductive dechlorination at station 3C. Another factor that may play some role is the difference between the penetration depths of the LACSD (gravity) and USGS (box) corers and their ability to recover surficial layers of the sediment (Lee and others, 2002).

Rates based on the whole-core inventories in the present study are lower than, by factors of approximately 2 to 4 , those reported for microcosm studies performed near or at the in situ temperature of the sediments at station $3 \mathrm{C}$ (namely, $0.151,0.23 \mathrm{yr}^{-1}$; table 6 ). The principal causes for this difference are that the microcosm experiments did not adequately mimic field conditions. For example, they did not have a continuing supply of sulfate. The lack of a continuing supply of sulfate means that this anion may (and in some cases is known to) have been exhausted in the microcosms during the period of incubation with the result that methanogenic conditions developed. Rates of dechlorination under methanogenic conditions are greater than under sulfidogenic conditions, so the rates seen in the microcosm experiments may not be characteristic of field conditions where sulfate is readily available. As mentioned earlier, Quensen and others $(2000,2001)$ observed sulfate at all depths in the cores at the time of collection, indicating that sulfate is available within the sediment column under field conditions. Secondly, aging of the spiked sediments was not performed. Microcosms were incubated immediately after introduction of the radiolabelled DDE. Whereas Quensen and others (2001) shook the contents of the vials prior to static incubation, no agitation or mixing was used in the method of Deming and Carpenter (2000). In either case, it is likely that binding of DDE to sedimentary organic matter was not the same in the microcosms as it is in the natural environment and that DDE was more available to the indigenous dechlorinating microorganisms than it is in the field. Numerous studies (see references in Alexander, 2000) have shown that if hydrophobic organic chemicals are added to soils or sediment and are allowed to age, they are less available for biodegradation than if no aging was allowed. Upon aging, these chemicals become less accessible to microorganisms capable of metabolizing them because of binding to or within highly sorptive phases in the sedimentary organic matter (Luthy and others, 1997). This explains why DDT and its transformation products in contaminated soils and sediments are often highly resistant to degradation (Morrison and others, 2000).

First-order loss rates for $\Sigma \mathrm{LAB}_{26}$ and 6- $\mathrm{C}_{12}$ calculated from inventories given in table 5 are 0.070 and $0.091 \mathrm{yr}^{-1}$, respectively. Because the decrease in concentration of LABs occurs at virtually all depths (see fig. 37) and because the concentrations and inventories of TAB3 and $\Sigma \mathrm{TAB}_{12}$ were essentially identical between the 1992 and 2003, the apparent loss of LABs must be attributable to in situ biodegradation. This in itself is an important finding as it has long been thought that the LABs are not degraded under anoxic conditions. The fact that there is little or no change in the I/E ratio with depth or between cores (fig. 35B) further indicates that the degradation pathway, operating under anoxic conditions, does not exhibit the same isomer selectivity that has been observed in oxic systems. This reinforces a caution issued by Eganhouse and others (2000) that using LABs for purposes of molecular stratigraphy in anoxic waste-contaminated sediments may be problematic. One other comment is in order. If degradation favored removal of the external isomers over internal isomers, one would expect the degradation rate of 6- $\mathrm{C}_{12}$ would be 
slower than for $\Sigma \mathrm{LAB}_{26}$. However, the reverse is seen for the 124B1 cores. This, again, signals a difference in pathway and possibly isomer selectivity.

Table 6. Comparison of DDE transformation rates in sediments of the Palos Verdes Shelf with results of relevant microcosm studies.

[yr ${ }^{-1}$, per year; $\mathrm{cm}$, centimeters; $\mathrm{g} / \mathrm{cm}^{2}$, grams per square centimeter; ${ }^{\circ} \mathrm{C}$, temperature in degrees Celsius]

\begin{tabular}{|c|c|c|c|c|c|}
\hline Station $^{\mathrm{a}}$ & Investigation & Period & Comments & $\begin{array}{c}\text { Transformation } \\
\text { rate }\left(\mathrm{yr}^{-1}\right)\end{array}$ & $\begin{array}{c}\text { Half-life } \\
\text { (years) }\end{array}$ \\
\hline \multicolumn{6}{|c|}{ Whole-core Inventories } \\
\hline 522 & present study & $1992-2003$ & & $0.051 \pm 0.006^{\mathrm{e}}$ & $13.6 \pm 1.7^{\mathrm{e}}$ \\
\hline $3 C$ & LACSD $^{b}$ & $1991-2005$ & & 0.085 & 8.1 \\
\hline $3 C$ & LACSD $^{b}$ & $1991-2005$ & & 0.058 & 12.0 \\
\hline $3 C$ & LACSD $^{b}$ & $1991-2005$ & & 0.047 & 14.6 \\
\hline $6 \mathrm{C}$ & LACSD $^{b}$ & $1991-2005$ & & 0.018 & 38.7 \\
\hline $6 \mathrm{C}$ & LACSD $^{b}$ & $1991-2005$ & & 0.028 & 24.9 \\
\hline $6 \mathrm{C}$ & LACSD $^{b}$ & $1991-2005$ & & 0.013 & 52.0 \\
\hline $3 \mathrm{C}$ & USGS/LACSD $^{c}$ & $1991-2005$ & Above $10 \mathrm{~g} / \mathrm{cm}^{2}$ & 0.089 & 7.8 \\
\hline $3 C$ & USGS/LACSD ${ }^{c}$ & $1991-2005$ & Below $10 \mathrm{~g} / \mathrm{cm}^{2}$ & 0.047 & 14.7 \\
\hline $6 \mathrm{C}$ & USGS/LACSD $^{\mathrm{c}}$ & $1991-2005$ & Above $10 \mathrm{~g} / \mathrm{cm}^{2}$ & 0.082 & 8.4 \\
\hline $6 \mathrm{C}$ & USGS/LACSD ${ }^{\mathrm{c}}$ & $1991-2005$ & Below $10 \mathrm{~g} / \mathrm{cm}^{2}$ & 0.019 & 36.5 \\
\hline \multicolumn{6}{|c|}{ Core-to-core } \\
\hline \multirow[t]{2}{*}{522} & present study & 1992-2003 & mean & $0.052 \pm 0.025^{\mathrm{e}}$ & $13.3 \pm 6.4^{\mathrm{e}}$ \\
\hline & & & median & 0.045 & 15.3 \\
\hline \multicolumn{6}{|c|}{ Microcosm studies } \\
\hline \multirow[t]{2}{*}{$3 \mathrm{C}$} & $\begin{array}{l}\text { Quensen and } \\
\text { others (2001) }\end{array}$ & $--^{d}$ & $10^{\circ} \mathrm{C}$ & 0.073 & 9.5 \\
\hline & & $--^{d}$ & $15^{\circ} \mathrm{C}$ & 0.151 & 4.6 \\
\hline $3 \mathrm{C}$ & Deming and Carpenter (2000) & 1997 & $30-40 \mathrm{~cm}, 12^{\circ} \mathrm{C}$ & 0.23 & 3 \\
\hline $6 \mathrm{C}$ & Deming and Carpenter (2000) & 1997 & $2-30 \mathrm{~cm}, 12^{\circ} \mathrm{C}$ & 0.087 & 8 \\
\hline $8 \mathrm{C}$ & Deming and Carpenter (2000) & 1997 & $2-22 \mathrm{~cm}, 12^{\circ} \mathrm{C}$ & 0.069 & 10 \\
\hline
\end{tabular}

${ }^{\mathrm{a}}$ Station 522 is near station $3 \mathrm{C}$.

${ }^{\mathrm{b}}$ Comparison of inventories for Los Angeles County Sanitation Districts (LACSD): a single core collected in 1991 with triplicate cores collected in 2005 (see table 5).

${ }^{\mathrm{c}}$ Analysis by C.R. Sherwood and B. Ferré (written commun., 2007) based on Los Angeles County Sanitation Districts (LACSD) data.

${ }^{\mathrm{d}}$ Not reported.

${ }^{\mathrm{e}}$ Maximum uncertainties estimated based on propagation of error analysis.

\section{Core-to-core comparison}

A vertical profile of the first-order rate coefficient $\left(\lambda^{\prime}{ }_{92}{ }^{\prime} 03\right)$ for $p, p$ '-DDE transformation along with aligned vertical concentration profiles of $p, p^{\prime}$-DDE and $p, p^{\prime}$-DDMU in the 124B1 cores is shown in figure 38. The mean rate is $0.052 \mathrm{yr}^{-1}$ and the median rate is $0.045 \mathrm{yr}^{-1}$. In general, rates are somewhat lower at shallower depths $\left(6\right.$ to $\left.28 \mathrm{~g} / \mathrm{cm}^{2}\right)$ and tend to increase irregularly with depth down to about $44 \mathrm{~g} / \mathrm{cm}^{2}$ after which a rapid decrease occurs. The vertical concentration profiles in panels $\mathrm{B}$ and $\mathrm{C}$ provide a means of visualizing what the concentrations of parent ( $p, p$ 'DDE) and product ( $p, p$ '-DDMU) were at coeval depths in the two cores. In the most heavily contaminated layers (about 30 to $45 \mathrm{~g} / \mathrm{cm}^{2}$ ), the rates are somewhat variable with higher values on the lower shoulder of the subsurface peak. 
Using a core collected at LACSD station 6C in 1997, Quensen and others (2001) found higher rates of dechlorination in surface sediments (less than $20 \mathrm{~cm}$ ) and at depth (greater than 40 $\mathrm{cm})$. The lower rates observed at intermediate depths $(24$ to $40 \mathrm{~cm})$ were attributed to the persistence of sulfate in these layers because dechlorination rates are lower in the presence of sulfate. The variation of in situ dechlorination rates shown in figure 38A does not show this pattern. This may either be a reflection of some difference between the locations of these stations (3C and $6 \mathrm{C}$ ) or an artifact of the microcosms, which did not have a continuing supply of sulfate. Microcosm experiments performed by Deming and Carpenter (2000) more closely mimicked natural conditions, but sulfate and methane concentrations were not monitored, so one does not know what the dominant TEAP was during the course of the experiment. They reported exceptionally high rates at depths of 30 to $40 \mathrm{~cm}$ for a core from station $3 \mathrm{C}$ (table 1 and 6). However, without data for the overlying sections, it is not possible to determine what the vertical profiles would have looked like. For cores collected from stations $6 \mathrm{C}$ and $8 \mathrm{C}$, highest rates were observed at depths of 2 to $30 \mathrm{~cm}$ and 2 to $22 \mathrm{~cm}$, respectively. This is partly consistent with the findings at 6C reported by Quensen and others (2001), but is completely opposite of the pattern observed in the present study. The reason for the latter difference is not immediately apparent, but it may, again, have to do with depletion of sulfate in the microcosms. Deming and Carpenter (2000) conducted an experiment where a core from 6C was spiked with seven times the usual amount of DDE. They found that sediments at depths of 24 to $36 \mathrm{~cm}$ had a higher than proportional response (in terms of dechlorination rate). They interpreted this as indicating that organisms at these depths were constrained in their metabolic activities, and hence, dechlorination rates, by limited access to nutrients and suitable substrates. However, this view must be tempered by the likelihood that with greater depth under field conditions, the possibility of sulfate depletion and the onset of methanogenesis would likely increase. In this context, it is worth mentioning that in many of the cores (see red bars in profiles shown in fig. 3, 15-18), particularly those collected from 2003-2005, $p, p^{\prime}$-DDMU abundance appears to decrease at depths near the $p, p^{\prime}$-DDE $+p, p^{\prime}-$ DDMU concentration peak and then increase on the lower shoulder of the subsurface peak. Thus, field data from several locations on the shelf are consistent with the rate profile shown in figure $38 \mathrm{~A}$.

Using LACSD biennial core data for the period 1991 to 2005, C.R.Sherwood and B. Ferré (written commun., 2007) computed $p, p^{\prime}$-DDE loss rates for two layers of the sediment column: 1) top layer (less than $\left.10 \mathrm{~g} / \mathrm{cm}^{2}\right)$, and 2) bottom layer $\left(10 \mathrm{~g} / \mathrm{cm}^{2}\right.$ and deeper). The premise of this approach is that the top layer represents an active surface zone that is subject to sedimentation, resuspension, mixing and in situ transformation reactions, all of which could result in apparent losses of $p, p$ '-DDE from the system. By contrast, losses from the bottom layer would largely be restricted to in situ transformations. At station 3C, near to but offshore from station 522 (where the 124B1 cores were collected; see fig. 5A-5B), the following first-order loss rates were determined by C.R.Sherwood and B. Ferré (written commun., 2007) using regression analysis: top layer = $0.089 \pm 0.011 \mathrm{yr}^{-1}$, bottom layer $=0.047 \pm 0.021 \mathrm{yr}^{-1}$ (table 6 ). The estimated loss rate in the bottom layer agrees quite well with the mean (or median) rates determined by direct comparison of the 124B1 cores. However, the rate for the top layer is almost twice that of the bottom layer. The difference in rates between the layers may be the result of an assumption that inventories within specified depth intervals at the surface of the sediment column $\left(10 \mathrm{~g} / \mathrm{cm}^{2}\right)$ can be compared. In fact, these depth intervals encompass sedimentary deposits having different average ages. The upper $10 \mathrm{~g} / \mathrm{cm}^{2}$ of later cores incorporates more solid mass from recent sedimentation than the upper $10 \mathrm{~g} / \mathrm{cm}^{2}$ of the earlier cores. Thus, higher rates in the top layer could result if more recently 
deposited sediment contained less-contaminated material which would serve to dilute the morecontaminated sediments deposited in earlier times. By comparison, the upper $10 \mathrm{~g} / \mathrm{cm}^{2}$ depth range of the 1991 core should incorporate a greater proportion of the deeper, older and more heavily contaminated sediments. The core alignment procedure used in the present work first adjusts the compaction-corrected depths of two cores based on the distribution of a persistent contaminant, such as PCB 153 or TAB3, and then compares coeval sediment intervals. It is acknowledged that error in the alignment procedure can result if the upper shoulder of the contaminant profile was 'eroded' by mixing during the period of interest (for example, 1992 to 2003). However, the amount of 'erosion' is limited by the numbers and activities of benthic infauna, both of which decrease rapidly with depth (Stull and others, 1996; Swift and others, 1996; Wheatcroft and Martin, 1996). The effect of such 'erosion' would be to raise the apparent rates in the vicinity of the upper shoulder and reduce them in the overlying sediment column. The lower rates seen at depths of 16 to $26 \mathrm{~g} / \mathrm{cm}^{2}$ and the slight increase in rates between 26 and $30 \mathrm{~g} / \mathrm{cm}^{2}$ in figure $38 \mathrm{~A}$ could be interpreted as being an effect of such 'erosion'. However, from existing data it seems very unlikely that intense mixing occurs below the upper $10 \mathrm{~cm}$ of the sediment column (Wheatcroft and Martin, 1994; Santschi and others, 2001). Below about $30 \mathrm{~g} / \mathrm{cm}^{2}$ one might expect that any such effects would be mitigated because redistribution of sediments is extremely unlikely. Average rates determined for this portion of the sediment column using the aligned 1992 and 2003 cores (greater than $30 \mathrm{~g} / \mathrm{cm}^{2}$ ) were $0.069 \mathrm{yr}^{-1}$. To investigate this question further, transformation rates were calculated based on a core profile offset of $3 \mathrm{~g} / \mathrm{cm}^{2}$. The mean rate was $0.052 \mathrm{yr}^{-1}$ and the median rate was $0.046 \mathrm{yr}^{-1}$, very close to those obtained with an offset of $4 \mathrm{~g} / \mathrm{cm}^{2}\left(0.052 \mathrm{yr}^{-1}\right.$ and $0.045 \mathrm{yr}^{-1}$, respectively). Moreover, the profiles showed the same pattern as illustrated in figure 38A. On this basis, it appears that the core alignment procedure is not a source of error in the estimation of mean or median rates or of the vertical variation in rates at this site. The effect of possible erosion of the upper shoulder of the $p, p$ '-DDE peak on these rates, if any, can only be surmised. However, the agreement between mean and median rates compared to rates based on the whole-core inventories suggests that the effects are negligible.

\section{Transformation pathway and products}

In this study, $p, p$ '-DDE, $p, p$ '-DDMU and $p, p^{\prime}$-DDNU, three putative members of a reductive dechlorination pathway (fig. 4), were measured in two cores. There is no direct proof that $p, p$ '-DDMU is being formed from $p, p^{\prime}$-DDE and that $p, p$ '-DDNU is being formed from $p, p$ 'DDMU in the field, but changes in the inventories (table 5) and vertical profiles of $p, p$ '-DDMU abundance (fig. 2-3) between 1991 and 2005 strongly support this concept. Another view of the three DDT compounds and their distribution in the two cores (after core alignment) is shown in figure 39. Scaling of the $\mathrm{x}$-axis is the same in these vertical concentration profiles for purposes of comparison. It is obvious that there is a systematic decrease in $p, p$ '-DDE concentrations and a systematic increase in $p, p^{\prime}$-DDMU and $p, p$ '-DDNU concentrations between 1992 and 2003. An interesting feature of the $p, p^{\prime}$-DDNU profile for the 2003 core is the peak at around 32 to $35 \mathrm{~g} / \mathrm{cm}^{2}$, which coincides nicely with the maximum concentrations for $p, p$ '-DDE and $p, p$ '-DDMU.

HRGC/MS analyses of the F2 fractions from extracts (see Methods section) of the 2003 core failed to reveal the existence of any other potential degradation products in the 2003 core. However, oxidation of the ethylene group of $p, p$ '-DDNU would likely lead to formation of $p, p$ 'DDA, $p, p$ '-DDOH and $p, p$ '-DBP (fig. 4). Eganhouse and others (2000) reported observing measurable (but unquantified) amounts of $p, p$ '-DBP (but not $p, p$ '-DDA and $p, p^{\prime}-\mathrm{DDOH}$ ) in selected F2 fractions of the 1992 core analyzed by EC-NCIMS. The inability to identify $p, p$ '-DBP 
in the F2 fractions of 2003 core by EI-HRGC/MS is difficult to explain. However, $p, p$ '-DDA and $p, p$ '-DDOH, if present, are most likely in the more polar F3 fraction, which has not yet been analyzed in either core. It is also possible that these compounds may have become bound to the nonextractable organic matter in the sediments (Schwarzbauer and others, 2003). In the case of $p, p$ '-DDM and $p, p$ '-DDA this has tentatively been confirmed.

In order to investigate the $p, p$ '-DDE $/ p, p$ '-DDMU/ $p, p$ '-DDNU system further and to assess whether $p, p$ '-DDNU is a dead-end product, summed molar inventories of these DDT compounds were compared in the 1992 and 2003 cores. The results, shown in figure 40, indicate that at almost all coeval depths, the sum of the molar inventories of $p, p^{\prime}$-DDE, $p, p$ '-DDMU, and $p, p^{\prime}$-DDNU in the 1992 core exceeds that in the 2003 core (panel A). One possible explanation for this difference is that there are degradation products not included in the mass balance. These products could arise through degradation of any of the three compounds ( $p, p^{\prime}$-DDE, $p, p^{\prime}$-DDMU, $p, p$ '-DDNU) by alternate pathways. Another possibility is the incorporation of these compounds as nonextractable residues in the sedimentary organic matter. However, experiments performed to date show no evidence of this phenomenon (Schwarzbauer, 2002). The difference between summed molar whole-core inventories of the 1992 and 2003 cores is 20.7 percent. This mass balance 'deficit' is very close to the level of analytical uncertainty (Eganhouse and others, 2000), so it is not possible to ascertain whether or not the 'deficit' is real. The difference can be plotted with depth in the core as shown in figure 40B. The magnitude of the 'deficit' varies markedly throughout the core, but the profile is similar in shape to that of the first-order transformation rate profile of $p, p$ '-DDE (fig. 38A). This suggests that the principal contributor to the 'deficit' is the difference between $p, p$ 'DDE concentrations, not those of $p, p$ '-DDMU or $p, p$ '-DDNU, in the two cores. Whether the mass balance deficit is real or not remains unresolved

\section{Environmental factors controlling DDE transformation rates}

The environmental factors that control the rate of $p, p$ '-DDE dechlorination in sediments of the Palos Verdes Shelf are poorly understood. Evidence comes from two sources: field data and laboratory microcosm experiments. The field data consist principally of the spatial distribution of p,p'-DDMU abundance as of 1992 (U.S. Department of Justice, 1994a), rates inferred from changes in $p, p$ '-DDE inventories over time (C.R. Sherwood and B. Ferré, written commun., 2007), and the vertical variation in rates at a single site near station $3 \mathrm{C}$ (this study). The laboratory microcosm data are from experiments performed by Quensen and others $(1998,2001)$ and Deming and Carpenter (2000).

From the limited data for whole-core inventories at stations occupied by the USGS and LACSD along the 60-meter isobath in 1991-1992 (fig. 20A-20B), a gradual but consistent trend is observed. Higher abundances of $p, p^{\prime}$-DDMU are found with increasing distance from the outfalls and with depth in the sediment column. By 2005, abundances of $p, p$ '-DDMU had increased at all locations but most at station 3C (fig. 20C). The analysis of LACSD core data offered by C.R. Sherwood and B. Ferré (written commun., 2007) for the various time intervals all starting in 1991 is consistent with this pattern. In microcosm studies, Quensen and others $(1998,2001)$ and Deming and Carpenter (2000) found a relationship between $p, p$ '-DDE dechlorination rate and distance from the outfall system. By comparing dechlorination rates in microcosms to which metabolizable organic matter had been added with rates in unamended microcosms, Quensen and others (2001) concluded that the availability of metabolizable organic matter was not responsible for the observed spatial differences in dechlorination rate. Rather, they attributed these trends to one or a combination of three factors: 1) differences in the microbial communities, 2) differences in the 
degree of inhibition of dechlorination activity, and 3) differences in the bioavailability of $p, p$ 'DDE.

Unfortunately, no data exist on the composition (or activities) of the microbial communities along the 60-meter isobath, so little can be said about this. However, it may be worth noting that Hampton and others (2002; see fig. 7 in that publication) describe the existence of active and inactive vents in the vicinity of station $3 \mathrm{C}$ that may serve as conduits for fluid phases to the water column. It is possible that these vents may be affecting the indigenous populations of dechlorinating microorganisms either through enhanced supply of necessary nutrients, substrates, and/or electron acceptors or through elevated temperature. As shown by Quensen and others (2001) the temperature dependence of dechlorination rates in microcosm studies can be described by the Arrhenius equation. Unfortunately, there are no measurements of in situ pore water chemistry or temperature at station $3 \mathrm{C}$ with which to test this hypothesis. Differences in the degree of inhibition of dechlorination activity could arise through competitive pressures or toxicity. The effluent-affected sediments of the Palos Verdes Shelf contain an extremely complex mixture of inorganic (for example, transition metals) and organic (PCBs, PAHS, DDT, etc...) contaminants. In general, concentrations of all contaminants in the subsurface effluent-affected sediment layers increase with proximity to the outfalls (Lee and others, 2002). The microcosm studies showed that dechlorination can occur at all locations, and, while rates vary with depth, throughout the sediment column. This is consistent with the occurrence of $p, p^{\prime}$-DDMU at all sub-bottom depths in cores along the 60-meter isobath and the increased abundance of $p, p$ '-DDMU at all depths between 1991 and 2005. However, it is conceivable, if not likely, that the activities of dechlorinating organisms are affected by the presence of effluent-derived contaminants, possibly including DDT.

Quensen and others (2001) found that dechlorination activity was highest in a core from station $6 \mathrm{C}$ at depths above $20 \mathrm{~cm}$ and below $40 \mathrm{~cm}$. They attributed the lower rates between 20 and $40 \mathrm{~cm}$ to delayed depletion of sulfate because dechlorination is slower in the presence of sulfate. Deming and Carpenter (2000) observed higher dechlorination rates in the upper portions of cores from stations $6 \mathrm{C}$ and $8 \mathrm{C}$ ( 2 to $30 \mathrm{~cm}, 2$ to $22 \mathrm{~cm}$, respectively). In an experiment where core sections from $6 \mathrm{C}$ were spiked with seven times the usual amount of ${ }^{14} \mathrm{C}$-labelled $p, p^{\prime}$-DDE, they found higher dechlorination rates and activity extending over a greater depth range. A nondimensional analysis of these results revealed that less than proportional responses occurred in the upper portion of the sediment column $(2$ to $22 \mathrm{~cm})$ and greater than proportional responses in the lower part of the sediment column ( 24 to $36 \mathrm{~cm}$ ). The authors hypothesized that the lower than proportional responses indicated that the bacterial colonies were approaching maximal rates of dechlorination; the higher than proportional responses in the more contaminated lower layer were thought to reflect adaptation to higher concentrations of DDE while the lower absolute rates of dechlorination in these sections suggested a limitation in the availability of metabolically required substrates and/or nutrients due to burial.

While it is not presently possible to reconcile the different vertical distributions of dechlorinating activity seen by Quensen and others (2001) and Deming and Carpenter (2000) in the $6 \mathrm{C}$ core, it seems likely that they stem from variations in experimental design. Moreover, in both sets of experiments, conditions were not truly reflective of the field, specifically with regard to the availability of sulfate and bioavailability of DDE. Quensen and others $(1998,2001)$ monitored the progress of sulfate depletion and onset of methanogenesis, whereas Deming and Carpenter (2000) apparently did not. Quensen and others (2001) reported that sulfate was still present in the sediment cores (down to $50 \mathrm{~cm}$ ) when they were collected, so it seems reasonable to assume that complete sulfate depletion does not occur naturally in the upper $50 \mathrm{~cm}$ of the sediment column at 
any of the stations along the 60-meter isobath. This was further confirmed for at least the upper 20 $\mathrm{cm}$ at station 6C (Van Cappellen and Santschi, 1999). If that is the case, dechlorination rates observed in microcosms that experienced sulfate depletion (and entered methanogenesis) are not applicable to existing field conditions. This suggests that other factors may explain the spatial variation in apparent dechlorination rate. One possibility is inhibition through toxicity. A consistent feature of the $p, p$ '-DDMU abundance profiles at stations other than $3 \mathrm{C}$ is a decline, sometimes quite rapid, in $p, p^{\prime}$-DDMU abundance at depths near the $p, p$ '-DDE $+p, p$ '-DDMU concentration maximum (see red bars in fig. $3,15-18$ ). This feature could indicate that at these stations, higher concentrations of effluent-derived contaminants inhibit dechlorination. Conversely, the lack of such a feature in profiles of cores from station $3 \mathrm{C}$ (for example fig. 2) may mean that the level of contamination is not sufficient at this location to inhibit dechlorination.

Concerning variations in bioavailability, it is difficult to offer any definitive statements that are supported by data. DDT and its transformation products were probably deposited in shelf sediments in association with fine-grained organic-rich particles from the waste effluent or possibly as discrete DDT waste particles derived from the Montrose plant (largely unaltered during primary waste treatment during the period of major DDT inputs). Dehydrochlorination of parent DDT and redistribution of the primary metabolite, $p, p$ '-DDE, among sediment phases and sediment pelletization (Drake and others, 2002) would have occurred subsequent to sedimentation. Along the 60-meter isobath sedimentation can be described as a time-varying spatial gradient in the relative fluxes of effluent and non-effluent particles to the sea floor. Consequently, the relative proportions of effluent-derived and non-effluent derived sedimentary organic matter varies spatially (laterally and vertically) as evidenced by the systematic distribution of stable carbon and nitrogen isotopes in sediment cores (Myers, 1974; Sweeney and others, 1980; Eganhouse and Kaplan, 1988) and the variation in sources of particles in the water column (Jones and others, 2002). It is possible that at locations removed from the outfall system where the influence of effluent-derived particulate matter was less (for example, station $3 \mathrm{C}$ ), the bioavailability of $p, p$ '-DDE is greater because of its association with organic matter of different character. Unfortunately, there are, at present, no data available to test this hypothesis.

Alternatively, the availability of $p, p$ '-DDE could vary spatially as a function of the degree of pelletization of the sediments. Drake and others (2002) found that organic carbon and $p, p$ '-DDE concentrations were highest in the finest size $(0$ to $20 \mu \mathrm{m})$ and in sand-sized (greater than $63 \mu \mathrm{m})$ fractions of non-disaggregated effluent-affected sediments from near station 6C. The sand-sized particles in the effluent-affected sediments at all locations were dominated by fecal pellets generated by benthic infauna. The proportion of aggregates was highest near the outfalls (approximately 30 percent) where Capitellid worms were present in large numbers, decreasing with increasing distance to the northwest (less than 10 percent near station 3C) owing to reduced Capitellid abundances. Because of the reduced surface area/volume and high organic carbon content of the aggregates, loss rates of $p, p$ '-DDE through desorption are slower than for finer sediment size fractions (Wiberg and Harris, 2002). Thus, release rates of $p, p$ '-DDE to surrounding pore water, where it can be dechlorinated to $p, p$ '-DDMU by microorganisms, is expected to be reduced when aggregate abundance is higher. In this regard, disaggregation of pellets through the activities of benthic infauna or physical processes could serve to increase the bioavailability of $p, p$ 'DDE. Thus, a spatial gradient in bioavailability, and hence dechlorination rates, could also reflect spatial variation in bioturbation and/or bio-irrigation intensities along the 60-meter isobath (Wheatcroft and Martin, 1996; Berelson and others, 2002). 
Finally, in comparing the vertical profile of $p, p$ '-DDE dechlorination rates at station $3 \mathrm{C}$ for the period 1992 to 2003 with the results of the microcosm experiments, no definitive conclusions can be drawn. This is due largely to the fact that most of these experiments were conducted using sediment cores nearer the outfall system where conditions do not appear to be as favorable for dechlorination. In addition, some of the experiments were driven to sulfate depletion which is unlikely to occur in the field (Quensen and others, 2001), whereas the dominant terminal electron accepting process in the experiments of Deming and Carpenter (2000) are unknown. The only microcosm data for station 3C obtained by Deming and Carpenter (2000) was for the lower portions of a single core, so little can be said concerning the vertical distribution of rates at $3 \mathrm{C}$ based on these experiments. Examination of figure 38A gives no indication of a relationship between dechlorination rate and contaminant concentration (as indicated by $p, p$ '-DDE).

Transformation rates increase and reach highest values within the 38 to $44 \mathrm{~g} / \mathrm{cm}^{2}$ interval. This is on the lower shoulder of the $p, p$ '-DDE contamination peak. Given the expected variability in profiles near this site, it is perhaps speculative to place too much emphasis on this feature. However, the abundance of $p, p$ '-DDMU also reaches highest levels below the $p, p$ '-DDE $+p, p$ 'DDMU concentration maximum in the 2005 replicate cores at station $3 \mathrm{C}$ (fig. 2). Thus, the higher rates observed at depth in the 124B1 cores (fig. 38A) directly contradict the hypothesis of Deming and Carpenter (2000) that greater burial results in a reduction in dechlorination rates through restriction of the availability of necessary substrates and/or nutrients. [Note: Deming and Carpenter's conclusions were reached based on a microcosm experiment with a core from station $6 \mathrm{C}$, not 3C.] This highlights the difficulty of drawing conclusions about $p, p$ '-DDE dechlorination rates obtained at one location and extrapolating them to other locations on the shelf. From existing information, it is seems very likely that multiple factors affect dechlorination rates on the Palos Verdes Shelf and these factors probably vary spatially and over time. Without further data, it will not be possible to predict, let alone enhance, these rates.

\section{Model of DDE $\rightarrow$ DDMU $\rightarrow$ DDNU transformation kinetics}

If one assumes that reductive dechlorination is the dominant pathway leading from $p, p$ 'DDE to $p, p$-DDNU and that $p, p$ '-DDNU is further degraded to as-yet-unidentified products, it is possible to model the time course of this transformation as a series of first-order decays. As noted earlier, the solution discovered by Bateman (1910) was applied to this problem. The main assumptions in the model are: 1) all reactions proceed by first-order kinetics, 2) whole-core inventories of the parent ( $p, p^{\prime}$ '-DDE) and daughter products ( $p, p^{\prime}$-DDMU, $p, p^{\prime}$-DDNU, unknown products) in the cores are known accurately, 3) no competing reactions are involved, 4) reaction rates do not change over time, and 5) no other processes are responsible for losses of these compounds from the sediments. No assumptions about the prior inventories of these compounds at this site are required.

The results of modeling this system for the time between collection of the 124B1 cores (1992 to 2003; panel A) and as projected forward by 100 years (panel B) are shown in figure 41. In this scenario, a mean rate coefficient of $0.052 \mathrm{yr}^{-1}$ was assumed for the $p, p$ '-DDE dechlorination step (from the mean rate obtained by direct core comparison; table 6). Rate coefficients of succeeding reactions (namely, $p, p$ '-DDMU to $p, p^{\prime}$-DDNU and $p, p$ '-DDNU to products unknown) were obtained by adjusting the rates, successively, until the model prediction matched the observed inventories (equation 9). Rates for the $p, p$ '-DDMU to $p, p$ '-DDNU and $p, p$ '-DDNU to 'products unknown' reactions were 0.070 and $0.161 \mathrm{yr}^{-1}$, respectively. As reductive dechlorination proceeds, there is an increase in the inventory of $p, p$ '-DDMU until 2002 after which inventories of this 
compound decline as the rate of $p, p^{\prime}$-DDNU production exceeds the rate of $p, p$ '-DDMU supply. According to this model scenario, $p, p$ '-DDNU inventories would peak in about 2014 and decline thereafter.

How do these model predictions relate to conditions at the site? One might start to answer this question by examining the assumptions given above. The actual order of these reactions and whether reaction order is uniform across the shelf and over time is, at present, unknown. Firstorder reaction kinetics were assumed (assumption 1) for purposes of the modeling in the absence of any evidence to the contrary, but there is no a priori reason to reject the possibility of higher or lower reaction orders, let alone spatial and/or temporal variation of reaction order.

Concerning the accuracy of the whole-core inventories (assumption 2), evidence has been presented for the performance of the method for determining $p$, $p$ '-DDE using SRM 1941a. The analytical results for both SRM samples were within the reported uncertainty in the NIST-certified concentrations. Unfortunately, NIST did not report certified concentrations for either $p, p$ '-DDMU or $p, p$ '-DDNU. Thus, there is no way of independently evaluating the accuracy of these measurements. One could argue, however, that because $p, p$ '-DDMU was supplied as part of a NIST-traceable mixture of seven DDT compounds that were used for purposes of instrument calibration (including both $p, p$ '-DDE and $p, p$ '-DDMU), if $p, p$ '-DDE was measured accurately as judged by the SRM 1941a analyses, $p, p$ '-DDMU would also have been quantified accurately, providing there was no evidence of interference. HRGC/MS analyses of the F2 fractions indicate that the peaks corresponding to $p, p^{\prime}$-DDMU and $p, p^{\prime}$-DDNU were interference-free.

The third assumption, that no competing reactions are involved, is more difficult to test directly. As noted above, the summed molar whole-core inventories of $p, p^{\prime}$-DDE, $p, p^{\prime}$-DDMU and $p, p$ '-DDNU in the two 124B1 cores were within 20 percent of each other. This is near the level of analytical uncertainty and appears to be largely attributable to differences in the $p, p$ '-DDE concentrations, but the possibility that this 'deficit' is real cannot be dismissed. The lack of any $p, p$-DDT in the sediments means that an in situ supply of $p, p$ '-DDE (via dehydrochlorination) no longer exists. Sources of $p, p^{\prime}$-DDMU and $p, p^{\prime}$-DDNU other than $p, p$ '-DDE and $p, p^{\prime}$-DDMU, respectively, can be postulated (fig. 4). However, production of $p, p$ '-DDMU from $p, p$ '-DDD and $p, p$ '-DDNU from $p, p$ '-DDMS seems unlikely for two reasons. First, the change in the molar inventory of $p, p$-DDD from 1992 to $2003\left(-0.014 \mu \mathrm{mol} / \mathrm{cm}^{2}\right)$ is insufficient to account for the increase in $p$, $p$ '-DDMU during this period $\left(+0.077 \mu \mathrm{mol} / \mathrm{cm}^{2}\right)$. The fact that $p, p$ '-DDMU was being transformed to $p, p$ '-DDNU at the same time means that the decrease in inventory of $p, p$ 'DDD can explain even less of the 'gross' increase in inventory of $p, p$ '-DDMU. Secondly, dehydrochlorination is believed to require oxidizing conditions, and except for surficial layers, these sediments are anoxic (Van Cappellen and Santschi, 1999). That is why reductive dechlorination is occurring in the first place. The same argument can be made for production of $p, p$ '-DDNU from $p, p$ '-DDMS, but because $p, p^{\prime}$-DDMS has not been quantified in these samples, the potential of this compound as a source of $p$, $p$-DDNU on the basis of molar whole-core inventory changes cannot be assessed.

The fourth assumption is that the rates in the model do not change with time. This is an assumption that is not likely to be correct. At or near station $3 \mathrm{C}$, rates of reductive dechlorination appear to have changed over the last decade to decade and a half. At present the cause for this change is unknown. It seems most likely that rates will change in the future, not only at or near station $3 \mathrm{C}$ but in other locations. However, whether, where, when, and how fast dechlorination rates will change cannot be predicted until the factors that control them are understood. Clearly this represents the greatest uncertainty in predictions of such a model. 
Finally, it is assumed in the model that no other processes are responsible for losses of parent or daughter products. Losses of these compounds due to the combined effects of mixing, resuspension, and desorption will occur at all locations along the shelf. However, the extent to which these processes result in losses within the sediment column at a given location is limited by the numbers, activities and distribution of resident infauna; the degree of pelletization of the sediment; and the frequency, intensity and duration of storm events. The bulk of the existing inventory of these DDT compounds in the most-contaminated sediments (for example, along the 60-meter isobath) is buried at depths below those where the most intense mixing and resuspension are likely to occur (fig. 14). Thus, in situ degradation at this location is expected to be one of the most important, if not the dominant, removal process for the foreseeable future (Sherwood and others, 2002). One mitigating factor is the presence and activities of thalassinid shrimp whose burrows can reach to depths where sediment contamination is high (Stull and others, 1996; Wheatcroft and Martin, 1994). In the vicinity of station 3C, these shrimp have been observed more frequently than at locations nearer the outfall. Transport of sediment from deep, heavily contaminated layers during burrow excavation would serve to mobilize these sediments and make them available for loss through the aforementioned processes. Judging from the results obtained in this study, such deep bioturbation did not occur in the cores collected in 1992 and 2003 near station $3 \mathrm{C}$.

\section{Conclusions}

In cooperation with the USEPA the USGS undertook a study of the rates of reductive dechlorination of $p, p^{\prime}$-DDE on the Palos Verdes Shelf, CA. In this project, available information on the distribution of DDT (especially $p, p^{\prime}$-DDE and $p, p^{\prime}$-DDMU) in Palos Verdes Shelf sediments and results of laboratory experiments on the reductive dechlorination of $p, p$ '-DDE were compiled. That information has been supplemented with chemical analyses of a core collected by the USGS near LACSD station $3 \mathrm{C}$ in 2003. This core was collected for purposes of comparing $p, p$ '-DDE inventories and concentrations in it with those in a core obtained from the same location in 1992. Taken together, the existing data provide a limited view of the spatial and temporal variation of reductive dechlorination on the Palos Verdes Shelf.

The dominant metabolite of DDT on the shelf remains $p, p$ '-DDE. However, in the last decade (1992 to 2003), the rate of dechlorination appears to have increased. In addition, field data clearly show that rates of reductive dechlorination increase with distance from (that is, to the northwest of) the outfall system. This trend is corroborated by three separate microcosm studies in which two different experimental approaches were used. The apparent recent increase in dechlorination rate noted above was greater for the station farthest from the outfalls (3C). The reasons for this spatial pattern are not understood, but the available data seem to indicate that biogeochemical transformation processes affecting $p, p^{\prime}$-DDE and other degradation products in shelf sediments are evolving. This may be in response to changing conditions of sedimentation due to reduced emission of solids from the LACSD outfalls. These changes have secondary effects, including alteration of the composition and activities of the benthic community and modification of carbon oxidation rates and, thus, redox conditions in the sediments (Stull and others, 1996). This may, in turn, influence reductive dechlorination rates on the shelf. It is also possible that the spatial differences reflect local features such as the presence of subsurface fluid vents or lower abundances of fecal pellets near station $3 \mathrm{C}$.

Comparison of the 1992 and 2003 cores suggests that transformation of $p, p^{\prime}$-DDE to $p, p^{\prime}$ DDMU via reductive dechlorination is an important, if not the predominant, loss mechanism near 
station 3C. Highest rates of reductive dechlorination at this site occur within depth intervals at the lower shoulder of the contaminant peak. Microcosm data for a complete core from station $3 \mathrm{C}$ is not available, but those obtained for other locations $(6 \mathrm{C}, 8 \mathrm{C})$ do not show the same downcore trends. This may, in part, be explained by the fact that the microcosm experiments do not accurately simulate in situ conditions, most importantly, access to seawater sulfate. Qualitatively, the microcosm experiments have provided data that is in agreement with field data in terms of the lateral but not vertical spatial variation in dechlorination rates. This likely reflects the fact that gradients in microbial and geochemical properties of sediments are much steeper vertically than they are laterally. To better understand this system and the factors that control reductive dechlorination rates on the shelf, more information would be needed on: 1) the composition and activities of dechlorinating (and non-dechlorinating) microorganisms in shelf sediments as well as factors that affect them, 2) the effects of in-place contaminants and their potential for toxic inhibition, 3) the bioavailability of $p, p^{\prime}$-DDE at different locations and sub-bottom depths in shelf sediments, and 4) the vertical variation in redox conditions at different locations. If undertaken, such studies would need to be conducted by an interdisciplinary team to ensure that the chemical and biological interactions are clearly delineated and understood.

In this study, a case has been made for the reductive dechlorination of $p, p$ '-DDE to $p, p$ 'DDMU, $p, p$ '-DDMU to $p, p$ '-DDNU and $p, p$ '-DDNU to products unknown. A model was presented that attempts to predict the inventories of these three DDT compounds near station $3 \mathrm{C}$ under specified conditions. Because $p, p$ '-DDMU concentrations are expected to exceed those of $p, p$-DDE within this decade (circa 2009), it would seem prudent to investigate the behavior of this transformation product and its toxicity to indigenous marine organisms. In the case of $p, p$ '-DDNU, concentrations may increase but they should remain substantially lower than those of $p, p$ '-DDMU for the foreseeable future. Consequently, the toxicity of $p, p$ '-DDNU may be of secondary concern. These statements depend on the assumption that transformation rates of the three compounds will remain constant. Evidence already in hand suggests that this assumption is probably unrealistic. This highlights the importance of understanding what controls dechlorination rates on the shelf. At present the ability to predict temporal and spatial changes in dechlorination is limited.

\section{Acknowledgments}

The authors are indebted to Carmen White (USEPA) for support during completion of this project. We also wish to thank Christopher Sherwood and Bénédicte Ferré of the USGS for offering substantial scientific input to the interpretations and help with an early version of the transformation model. We are grateful for data and relevant information that were supplied by Peter Dartnell, Brian Edwards, Homa Lee, Robert Kayen, and Kurt Rosenberger of the USGS, ChiLi Tang and Joseph Meistrell of the LACSD, Ann Hurley of the USDOJ, and Charles Philips of SAIC. Additional thanks are due to Brian Edwards, Homa Lee, and the scientific staff and crew of the USGS A-1-03-SC cruise for collecting and shipping the 2003 sediment core. Jurate Landwehr assisted with propagation of error calculations. Finally, we wish to thank Mary Jo Baedecker of the USGS, and Keith Maruya and two unknown reviewers from SCCWRP for providing thoughtful comments on an early version of this report. This work was supported by the U.S. Environmental Protection Agency (USEPA/USGS IAG \#DW-14-95561601). 


\section{References Cited}

Aislabie, J.M., Richards, N.K., and Boul, H.L., 1997, Microbial degradation of DDT and its residues-a review: New Zealand Journal of Agricultural Research, v. 40, p. 269-282.

Alexander, M., 2000, Aging, bioavailability, and overestimation of risk from environmental pollutants: Environmental Science \& Technology, v. 34, no. 20, p. 4259-4265.

Anderson, D.W., Jehl, J.R., Risebrough, R.W., Woods, L.A., Deweese, L.R., and Edgecomb, W.G., 1975, Brown pelicans-improved reproduction off the southern California coast: Science, v. 198, p. 806-808.

Bailey, A., and Costa, H.J., 1994, Chemical analyses of sediment cores data report: in U.S.

Department of Justice, Southern California Bight Natural Resource Damage Assessment Expert Reports, 1994, chap. 2, appendix H, 138 p.

Bascom, W., Mardesich, J. and Stubbs, H., 1982, An improved corer for soft sediments: in U.S. Department of Justice, Southern California Coastal Water Research Project, Biennial Report 1981-1982, Long Beach, CA, p. 267-271.

Bateman, H., 1910, The solution of a system of differential equations occurring in the theory of radio-active transformations: Proceedings of the Cambridge Philosophical Society, v. XV, part V, p. 423-427.

Bayona, J.M., Albaigés, J., Solanas, A.M., and Grifoll, M., 1986, Selective aerobic degradation of linear alkylbenzenes by pure microbial cultures: Chemosphere, v. 15, no. 5, p. 595-598.

Bedard, D.L., and Quensen, J.F., 1995, Microbial reductive dechlorination of polychlorinated biphenyls: in Young, L.Y., and Cerniglia, C.E., eds., Microbial transformation and degradation of toxic organic chemicals: New York, Wiley-Liss, p. 127-216.

Berelson, W.M., Johnson, K., Coale, K., and Li, H.-C., 2002, Organic matter diagenesis in the sediments of the San Pedro Shelf along a transect affected by sewage effluent: Continental Shelf Research, v. 22, p. 1101-1115.

Bhatia, M., and Singh, H.D., 1996, Biodegradation of commercial linear alkyl benzenes by Nocardia amarae: Journal of Bioscience, v. 21, no. 4, p. 487-496.

Brown, J.F., and Wagner, R.E., 1990, PCB movement, dechlorination, and detoxication in the Acushnet estuary: Environmental Toxicology and Chemistry, v. 9, p. 1215-1233.

Bzdusek, P.A., Christensen, E.R., Lee, C.M., Pakdeesusuk, U., and Freedman, D.L., 2006, PCB congeners and dechlorination in sediments of Lake Hartwell, South Carolina, determined from cores collected in 1987 and 1998: Environmental Science \& Technology, v. 40, no. 1, p. 109-119.

Callahan, M.A., Slimak, M.W., Gabel, N.W., May, I.P., Fowler, C.F., Freed, J.R., Jennings, P., Durfee, R.L., Whitmore, F.C., Maestri, B., Mabey, W.R., Holt, B.R., and Gould, C., 1979, Water-related environmental fate of 129 priority pollutants, Volume I- Introduction and technical background, metals, and inorganics, pesticides and PCBs: U.S. Environmental Protection Agency, EPA-440/4-79-029a [variously paged].

Carry, C.W., and Redner, J.A., 1970, Pesticides and heavy metals: County Sanitation Districts of Los Angeles County, Progress Report December 1970, 51 p.

Chartrand, A., 1986, Montrose Chemical Corporation-strategies for managing a widespread point source contaminant: in Proceedings of the symposium, Managing Inflows to California's Bays and Estuaries, Monterey, CA., p. 50-56.

Chartrand, A.B., Moy, S., Safford, A.N., Yoshimura, T., and Schinazi, L.A., 1985, Ocean dumping under Los Angeles Regional Water Quality Control Board permit-A review of past practices, potential adverse impacts, and recommendations for future actions: Los Angeles, California Regional Water Quality Control Board, 36 p., 2 appendices. 
Cochran, J.W., and Frame, G.M., 1999, Recent developments in the high-resolution gas chromatography of polychlorinated biphenyls: Journal of Chromatography A, v. 843, p. 323-368.

Deming, J.W., and Carpenter, S.D., 2000, DDE dechlorination under in situ conditions in continental shelf sediments: U.S.D.C. Case No. CV 90-3122-R, United States, et al., v. Montrose et al., Defendant Exhibit No. 9204, 17 p.

Drake, D.E., Sherwood, C.R., and Wiberg, P.L., 1994, Predictive modeling of the natural recovery of the contaminated effluent-affected sediment, Palos Verdes margin, southern California: in U.S. Department of Justice, Southern California Bight Natural Resource Damage Assessment Expert Reports, 1994, chap. 20, 93 p.

Eganhouse, R.P. and Venkatesan, M.I., 1993, Chemical oceanography and geochemistry: in Dailey, M.D., and others, eds., Ecology of the Southern California Bight: Berkeley, CA, University of California Press, p. 71-189.

Eganhouse, R.P., 2004, Molecular markers and their use in environmental organic geochemistry: in Hill, R.J., and others, eds., Geochemical investigations in earth and space science-A tribute to Isaac R. Kaplan, The Geochemical Society Special Publication Series 9: Amsterdam, Elsevier, p. 143-158.

Eganhouse, R.P., and Kaplan, I.R., 1982, Extractable organic matter in municipal wastewaters. 2. Hydrocarbons-molecular characterization, Environmental Science \& Technology: v. 16, p. 541551.

Eganhouse, R.P., and Kaplan, I.R., 1988, Depositional history of Recent sediments from San Pedro Shelf, California-Reconstruction using elemental abundance, isotopic composition and molecular markers: Marine Chemistry, v. 24, p. 163-191.

Eganhouse, R.P., and Pontolillo, J., 2000, Depositional history of organic contaminants on the Palos Verdes Shelf, California: Marine Chemistry, v. 70, p. 317-338.

Eganhouse, R.P., Blumfield, D.L., and Kaplan, I.R., 1983b, Long-chain alkylbenzenes as molecular tracers of domestic wastes in the marine environment: Environmental Science \& Technology, v. 17, p. 523-530.

Eganhouse, R.P., Pontolillo, J., and Leiker, T.J., 2000, Diagenetic fate of organic contaminants on the Palos Verdes Shelf, California: Marine Chemistry, v. 70, p. 289-315.

Eganhouse, R.P., Ruth, E.C., and Kaplan, I.R., 1983a, Determination of long-chain alkylbenzenes in environmental samples by argentation thin-layer chromatography/high-resolution gas chromatography and gas chromatography/mass spectrometry: Analytical Chemistry, v. 55, p. 2120-2126.

Eganhouse, R.P., Simoneit, B.R.T., and Kaplan, I.R., 1981, Extractable organic matter in urban stormwater runoff. 2. Molecular characterization: Environmental Science \& Technology, v. 15, p. 315-326.

Esaac, E.G., and Matsumura, F., 1980, Metabolism of insecticides by reductive systems:

Pharmacology and Therapeutics, v. 9, p. 1-26.

Farley, K.J., 1990, Predicting organic accumulation in sediments near marine outfalls: Journal of Environmental Engineering, v. 116, no. 1, p.144-165.

Frame, G.M., 1997a, A collaborative study of 209 PCB congeners and 6 Aroclors on 20 different HRGC columns. 1. Retention and coelution database: Fresenius Journal of Analytical Chemistry, v. 357, p. 701-713.

Frame, G.M., 1997b, A collaborative study of 209 PCB congeners and 6 Aroclors on 20 different HRGC columns. 2. Semi-quantitative Aroclor congener distributions: Fresenius Journal of Analytical Chemistry, v. 357, p. 714-722. 
Frame, G.M., Cochran, J.W., and Bøwadt, S.S., 1996, Complete PCB congener distributions for 17 Aroclor mixtures determined by 3 HRGC systems optimized for comprehensive, quantitative, congener-specific analysis: Journal of High Resolution Chromatography, v. 19, p. 657-668.

Gann, J.T., 1992, YoNav-Your own integrated navigation system for DOS platforms: U.S. Geological Survey Open-File Report 92-565, 62 p.

Guitart, R., Puig, P., and Gómez-Catalán, J., 1993, Requirement for a standardized nomenclature criterion for PCBs-computer-assisted assignment of correct congener denomination and numbering: Chemosphere, v. 27, no. 8, p. 1451-1459.

Haller, H.L., Bartlett, P.D., Drake, N.L., Newman, M.S., Cristol, S.J., Eaker, C.M., Hayes, R.A., Kilmer, G.W., Magerlein, B., Mueller, G.P., Schneider, A., and Wheatley, W., 1945, The chemical composition of technical DDT: Journal of the American Chemical Society, v. 67, p. 1591-1602.

Hamer, M.R., 1994, Processing of navigation and bathymetric data collected on the Palos Verdes margin: in U.S. Department of Justice, Southern California Bight Natural Resource Damage Assessment Expert Reports, 1994, chap. 2, appendix I, 21 p.

Hampton, M.A., Karl, H.A., and Murray, C.J., 2002, Acoustic profiles and images of the Palos Verdes margin-implications concerning deposition from the White's Point outfall: Continental Shelf Research, v. 22, p. 841-857.

Hartmann, P.C., Quinn, J.G., King, J.W., Tsutsumi, S., and Takada, H., 2000, Intercalibration of LABs in marine sediment SRM1941a, and their application as a molecular marker in Narragansett Bay sediments: Environmental Science \& Technology, v. 34, no. 5, p. 900-906.

Hedges, J.I, and Prahl, F.G., 1993, Early diagenesis-Consequences for application of molecular biomarkers: in Engel, M.H., and Macko, S.A., eds., Organic Geochemistry- Principles and applications: New York, Plenum Press, p. 237-253.

Hedges, J.I., and Stern, J.H., 1984, Carbon and nitrogen determinations of carbonate-containing solids: Limnology and Oceanography, v. 29, p. 657-663.

Hendricks, T., and Eganhouse, R., 1992, Modification and verification of sediment deposition models: Sacramento, Final Report to the Regional Water Quality Control Board, State of California, $330 \mathrm{p}$.

Hons, G., 1995, Alkylarylsulfonates-History, manufacture, analysis, and environmental properties: in Stache, H.W., ed., Anionic Surfactants-Organic Chemistry: New York, Marcel Dekker, p. 39108.

Ishiwatari, R., Takada, H., and Yun, S.-J., 1983, Alkylbenzene pollution of Tokyo Bay sediments: Nature, v. 201, no. 5901, p. 599-600.

Johnson, S.J., Barry, D.A., Christofi, N., and Patel, D., 2001, Potential for anaerobic biodegradation of linear alkylbenzene cable oils-Literature review and preliminary investigation: Land Contamination \& Reclamation, v. 9, no. 3, p. 279-291.

Jones, B.H., Noble, M.A., and Dickey, T.D., 2002, Hydrographic and particle distributions over the Palos Verdes Continental Shelf-spatial, seasonal and daily variability: Continental Shelf Research, v. 22, p. 945-965.

Kayen, R.E., Edwards, B.D., and Lee, H.J., 1999, Nondestructive laboratory measurement of geotechnical and geoacoustic properties through intact core-liner: in Marr, W.A., and Fairhurst, C.E., eds., Nondestructive and automated testing for soil and rock properties: West Conshohocken, PA, American Society for Testing and Materials, ASTM STP 1350, p. 83-94. Kayen, R.E., Lee, H.J., and Hein, J.R., 2002, Influence of the Portuguese Bend landslide on the character of the effluent-affected sediment deposit, Palos Verdes margin: southern California, Continental Shelf Research, v. 22, p. 911-922. 
Klein, D.H., and Goldberg, E.D., 1970, Mercury in the marine environment: Environmental Science \& Technology, v. 4, p. 765-768.

Kolpack, R.L., ed., 1987, Environmental processes affecting DDT contaminated sediment off Palos Verdes, California-A sediment dynamics workshop sponsored by the LACSD: County Sanitation Districts of Los Angeles County, 1987, 102 p.

Kuipers, B., Cullen, W.R., and Mohn, W.W., 1999, Reductive dechlorination of nonachlorobiphenyls and selected octachlorobiphenyls by microbial enrichment cultures: Environmental Science \& Technology, v. 33, no. 20, p. 3579-3585.

Lal, R., and Saxena, D.M., 1982, Accumulation, metabolism, and effects of organochlorine insecticides on microorganisms: Microbiological Reviews, v. 46, no. 1, p. 95-127.

Lamoureux, E.M., Brownawell, B.J., and Bothner, M.H., 1996, Linear alkylbenzenes as tracers of sewage-sludge-derived inputs of organic matter, PCBs, and PAHs to sediments at the 106-Mile Deep Water Disposal Site: Journal of Marine Environmental Engineering, v. 2, p. 325-342.

Lee, H. J., and Wiberg, P.L., 2002, Sedimentation processes, DDT, and the Palos Verdes margin: Continental Shelf Research, v. 22, nos. 6-7, p. 835-1115.

Lee, H.J., 1994a, The distribution and character of contaminated effluent-affected sediment, Palos Verdes margin, Southern California: in U.S. Department of Justice, Southern California Bight Natural Resource Damage Assessment Expert Reports, 1994, chap. 2, 261 p. 1994b, County Sanitation Districts of Los Angeles County (LACSD) multi-year measurements of chemical constituents and bulk density of sediments on the Palos Verdes margin: in U.S. Department of Justice, Southern California Bight Natural Resource Damage Assessment Expert Reports, 1994, chap. 2, appendix F, 158 p.

Lee, H.J., and Kayen, R.E., 1994, USGS coring, core handling, and core testing of sediment on the Palos Verdes margin: in U.S. Department of Justice, Southern California Bight Natural Resource Damage Assessment Expert Reports, 1994, chap. 2, appendix J, 15 p.

Lee, H.J., Sherwood, C.R., Drake, D.E., Edwards, B.D., Wong, F., Hamer, M., 2002, Spatial and temporal distribution of contaminated, effluent-affected sediment on the Palos Verdes margin, southern California: Continental Shelf Research, v. 22, p. 859-880.

Li, N., Wania, F., Lei, Y.D., and Daly, G.L., 2003, A comprehensive and critical compilation, evaluation, and selection of physical-chemical property data for selected polychlorinated biphenyls: Journal of Physical Chemical Reference Data, v. 32, no. 4, p. 1545-1590.

List, E.J., 1997, Biodegradation of DDT/DDE in Palos Verdes sediments: Submitted to the U.S. Environmental Protection Agency, Palos Verdes Technical Advisory Committee, April 1997 [variously paged].

Los Angeles County Sanitation Districts, 2005, Palos Verdes Ocean Monitoring Annual Report 2005, 502 p.

Luthy, R.G., Aiken, G.R., Brusseau, M.L., Cunningham, S.D., Gschwend, P.M., Pignatello, J.J., Reinhard, M., Traina, S.J., Weber, W.J., and Westall, J.C., 1997, Sequestration of hydrophobic organic contaminants by geosorbents: Environmental Science \& Technology, v. 31, no. 12, p. 3341-3347.

MacGregor, J.S., 1974, Changes in the amount and proportions of DDT and its metabolites, DDE and DDD, in the marine environment off southern California, 1949-1972: Fishery Bulletin, v. 72, no. 2, p. 275-293.

MacGregor, J.S., 1976, DDT and its metabolites in the sediments off southern California: Fishery Bulletin, v. 74, no. 1, p. 27-35.

Macko, S.A., Engel, M.H., and Parker, P.L., 1993, Early diagenesis of organic matter in sedimentsAn assessment of mechanisms and preservation by the use of isotopic molecular approaches: in 
Engel, M.H., and Macko, S.A., eds., Organic Geochemistry- Principles and applications: New York, Plenum Press, p. 211-224.

Magar,V.S., Brenner, R.C., Johnson, G.W., and Quensen, J.F., 2005b, Long-term recovery of PCB-contaminated sediments at the lake Hartwell Superfund site-PCB dechlorination. 2. Rates and extent: Environmental Science \& Technology, v. 39, no. 10, p. 3548-3554.

Magar,V.S., Johnson, G.W., Brenner, R.C., Quensen, J.F., Foote, E.A., Durell, G., Ickes, J.A., and Peven-McCarthy, C., 2005a, Long-term recovery of PCB-contaminated sediments at the lake Hartwell Superfund site-PCB dechlorination. 1. End-member characterization: Environmental Science \& Technology, v. 39, no. 10, p. 3538-3547.

Manen, C-A., 1994, Southern California Damage Assessment Analytical Chemistry Quality Assurance Plan: in U.S. Department of Justice, Southern California Bight Natural Resource Damage Assessment Expert Reports, 1994, chap. 1, appendix 1, 42 p.

Manen, C-A., Bailey, A., and Baldwin, J.K., 1994, Quality assurance for the Southern California Natural Resource Damage Assessment: in U.S. Department of Justice, Southern California Bight Natural Resource Damage Assessment Expert Reports, 1994, chap. 1, 14 p.

Martens, C.S., Haddad, R.I., and Chanton, J.P., 1992, Organic matter accumulation, remineralization, and burial in an anoxic coastal sediment: in Whelan, J., and Farrington, J.W., eds., Organic matter-Productivity, accumulation, and preservation in Recent and ancient sediments: New York, Columbia University Press, p. 82-98.

Massé, R., Lalanne, D., Messier, F., and Sylvestre, M., 1989, Characterization of new bacterial transformation products of 1,1,1-trichloro-2,2-bis-(4-chlorphenyl) ethane (DDT) by gas chromatography/mass spectrometry: Biomedical and Environmental Mass Spectrometry, v. 18, p. 741-752.

McDermott, D.J., Heesen, T.C., Young, D.R., 1974, DDT in bottom sediments around five southern California outfall systems: El Segundo, CA, Southern California Coastal Water Research Project, Technical Memorandum 217, 54 p.

Modler, R., 1994, Stanford Research Institute, Menlo Park, CA (personal communication).

Morrison, D.E., Robertson, B.K., and Alexander, M., 2000, Bioavailability to earthworms of aged DDT, DDE, DDD, and dieldrin in soil: Environmental Science \& Technology, v. 34, no. 4, p. 709-713.

Myers, E.P., 1974, The concentration and isotopic composition of carbon in marine sediments affected by a sewage discharge: Pasadena, California Institute of Technology, Ph.D. dissertation, 179 p.

National Academy of Sciences, 2001, A Risk-Management Strategy for PCB-contaminated Sediments: Washington, D.C., National Academy Press, 432 p.

Niedoroda, A.W., Swift, D.J.P., Reed, C.W., and Stull, J.K., 1996, Contaminant dispersal on the Palos Verdes continental margin: III. Processes controlling transport, accumulation and reemergence of DDT-contaminated sediment particles: The Science of the Total Environment, v. 179, p. 109-133.

Noble, M.A., Ryan, H.F., and Wiberg, P.L., 2002, The dynamics of subtidal poleward flows over a narrow continental shelf, Palos Verdes, CA: Continental Shelf Research, v. 22, p. 923-944.

Ötvös, I., Iglewski, S., Hunneman, D.H., Bartha, B., Balthazár, Z., and Pályi, G., 1973, Petrochemical analytical problems II. Gas-liquid chromatographic-mass spectrometric investigation of industrial dodecylbenzenes: Journal of Chromatography, v. 78, p. 309-316.

Pakdeesusuk, U., Lee, C.M., Coates, J.T., and Freedman, D.L., 2005, Assessment of natural attenuation via in situ reductive dechlorination of polychlorinated biphenyls in sediments of the Twelve Mile Creek arm of Lake Hartwell, SC: Environmental Science \& Technology, v. 39, no. 4, p. 954-952. 
Palekar, L.D., Maruya, K.A., Kostka, J.E., and Wiegel, J. 2003, Dehalogenation of 2,6dibromobiphenyl and 2,3,4,5,6-pentachlorobiphenyl in contaminated estuarine sediment: Chemosphere, v. 53, p. 593-600.

Peters, K.E., Walters, C.C., and Moldowan, J.M., 2005, The Biomarker Guide-Second Edition: Cambridge, Cambridge University Press, 1155 p.

Quensen, J.F., and Tiedje, J.M., 1997, Evidence for the microbial degradation of DDE: Submitted to the U.S. Environmental Protection Agency, Palos Verdes Technical Advisory Committee, April 1997, 12 p.

Quensen, J.F., Boyd, S.A., and Tiedje, J.M., 1990, Dechlorination of four commercial polychlorinated biphenyl mixtures (Aroclors) by anaerobic microorganisms from sediments: Applied and Environmental Microbiology, v. 56, no. 8, p. 2360-2369.

Quensen, J.F., Mueller, S.A., Jain, M.K., and Tiedje, J.M., 1998, Reductive dechlorination of DDE to DDMU in marine sediment microcosms: Science, v. 280, p. 722-724.

Quensen, J.F., Tiedje, J.M., Jain, M.K., and Mueller, S.A., 2001, Factors controlling the rate of DDE dechlorination to DDMU in Palos Verdes margin sediments under anaerobic conditions: Environmental Science \& Technology, v. 35, no. 2, p. 286-291.

Quensen, J.F., Tiedje, J.M., Jain, M.K., and Mueller, S.A., 2000, Factors controlling the rate of DDE dechlorination to DDMU in Palos Verdes margin sediments under anaerobic conditions: : U.S.D.C. Case No. CV 90-3122-R, United States, et al., v. Montrose et al., Defendant Expert Report, $31 \mathrm{p}$.

Raymundo, C.C., and Preston, M.R., 1992, The distribution of linear alkylbenzenes in coastal and estuarine sediments of the western North Sea: Marine Pollution Bulletin, v. 24, no. 3, p. 138-146.

Redner, J.A., and Payne, K., 1971, Chlorinated hydrocarbons: County Sanitation Districts of Los Angeles County, Progress Report-December 1971, 78 p.

Rochkind-Dubinsky, M.L., Sayler, G.S., and Blackburn, J.W., 1987, Microbiological decomposition of chlorinated aromatic compounds: New York, Marcel Dekker, Microbiological series, Vol. 18, 313 p.

Santschi, P.H., Guo, L., Asbill, S., Allison, M., Kepple, A.B., and Wen, L.-S., 2001, Accumulation rates and sources of sediments and organic carbon on the Palos Verdes shelf based on radioisotopic tracers $\left({ }^{137} \mathrm{Cs},{ }^{239,240} \mathrm{Pu},{ }^{210} \mathrm{~Pb},{ }^{234} \mathrm{Th},{ }^{238} \mathrm{U}\right.$ and $\left.{ }^{14} \mathrm{C}\right)$ : Marine Chemistry, v. 73, p. 125152.

Schantz, M.M., Benner, B.A., Hays, M.J., Kelly, W.R., Vocke, R.D., Demiralp, R., Greenberg, R.R., Schiller, S.B., Lauenstein, G.G., and Wise, S.A., 1995, Certification of standard reference material (SRM) 1941a, organics in marine sediment: Fresenius Journal of Analytical Chemistry, v. 352, p. 166-173.

Schulz, D.E., Petrick, G., and Duinker, J.C., 1989, Complete characterization of polychlorinated biphenyl congeners in commercial Aroclor and Clophen mixtures by multidimensional gas chromatography-electron capture detection: Environmental Science \& Technology, v. 23, no. 7, p. 852-859.

Schwarzbauer, J., 2002, Aachen University of Technology, Aachen, Germany (personal communication).

Schwarzbauer, J., Ricking, M., Littke, R., 2003, DDT-related compounds bound to the nonextractable particulate matter in sediments of the Teltow Canal, Germany: Environmental Science \& Technology, v. 37, no. 3, p. 488-495.

Science Applications International Corporation, 2005, Final report for the summer 2004 sediment displacement study on the Palos Verdes Shelf, 137 p. 
Shen, L., and Wania, F., 2005, Compilation, evaluation, and selection of physical-chemical property data for organochlorine pesticides: Journal of Chemical \& Engineering Data, v. 50, no. 3, p. 742-768.

Sherblom, P.M., Gschwend, P.M., and Eganhouse, R.P., 1992, Aqueous solubilities, vapor pressures, and 1-octanol-water partition coefficients for C9-C14 linear alkylbenzenes: Journal of Chemical \& Engineering Data, v. 37, no. 4, p. 394-399.

Sherwood, C.R., Drake, D.E., Wiberg, P.L., and Wheatcroft, R.A., 2002, Prediction of the fate of $p, p$ '-DDE in sediment on the Palos Verdes shelf, California, USA: Continental Shelf Research, v. 22, p. 1025-1058.

Steber, J., Herold, C.-P., and Limia, J.M., 1995, Comparative evaluation of anaerobic biodegradability of hydrocarbons and fatty derivatives currently used as drilling fluids: Chemosphere, v. 31, no. 4, p. 3105-3118.

Stull, J.K., Swift, D.J.P., and Niedoroda, A.W., 1996, Contaminant dispersal on the Palos Verdes continental margin-I. Sediments and biota near a major California wastewater discharge: The Science of the Total Environment, v. 179, p. 73-90.

Sweeney, R.E., Kalil, E.K., and Kaplan, I.R., 1980, Characterization of domestic and industrial sewage in southern California coastal sediments using nitrogen, carbon, sulphur and uranium tracers: Marine Environmental Research, v. 3, p. 225-243.

Swift, D.J.P., Stull, J.K., Niedoroda, A.W., Reed, C.W., and Wong, G.T.F., 1996, Contaminant dispersal on the Palos Verdes continental margin-II. Estimates of the biodiffusion coefficient, $D_{B}$, from composition of the benthic infaunal community: The Science of the Total Environment, $v$. 179, p. 91-107.

Swisher, R.D., 1987, Surfactant biodegradation: New York, Marcel Dekker, Surfactant Science Series, v. 18, 1085 p.

Takada, H., and Eganhouse, R.P., 1998, Molecular markers of anthropogenic waste: in Meyers, R.A., ed., Encyclopedia of Environmental Analysis and Remediation: New York, Wiley, p. 28832938.

Takada, H., and Ishiwatari, R., 1987, Linear alkylbenzenes in urban riverine environments in Tokyo-Distribution, source, and behavior: Environmental Science \& Technology, v. 21, no., 9, p. 875-883.

Takada, H., and Ishiwatari, R., 1989, Biodegradation experiments of linear alkylbenzenes (LABs): Isomeric composition of $\mathrm{C} 12 \mathrm{LABs}$ as an indicator of the degree of LAB degradation in the aquatic environment: Environmental Science \& Technology, v. 24, no. 1, p. 86-91.

U.S. Army Corps of Engineers, 2002, Field pilot study of in situ capping of Palos Verdes Shelf contaminated sediments, September 2002, 248 p.

U.S. Department of Justice, 1994a, Southern California Bight Natural Resource Damage Assessment Expert Reports. , 1994b, Standard operating procedures used by ADL and GERG: in Southern California Bight Natural Resource Damage Assessment Expert Reports, 1994, chap. 1, appendix 2, 205 p. 1994c, Data validation and data management: in Southern California Bight Natural Resource Damage Assessment Expert Reports, 1994, chap. 1, appendix 3, 5 p.

U.S. Environmental Protection Agency, 1992, Definition and procedure for the determination of the method detection limit, Revision 1.11, Appendix B of 40 CFR, Part 136, p. 565-567.

Van Cappellen, P., and Santschi, P. H., 1999, Organic matter degradation and bioirrigation in marine sediments impacted by wastewater outfall (Pals Verdes Shelf, Southern California Bight): U.S.D.C. Case No. CV 90-3122-R, United States, et al., v. Montrose et al., Defendant Expert Report, 45 p. 
Venkatesan, M.I., Greene, G.E., Ruth, E., and Chartrand, A.B., 1996, DDTs and dumpsite in the Santa Monica Basin, California: The Science of the Total Environment, v. 179, p. 61-71.

Wheatcroft, R.A., and Martin, W.R., 1994, Solid-phase bioturbation processes on the Palos Verdes Shelf: in U.S. Department of Justice, Southern California Bight Natural Resource Damage Assessment Expert Reports, 1994, chap. 20, appendix E, 228p.

Wheatcroft, R.A., and Martin, W.R., 1996, Spatial variation in short-term $\left({ }^{234} \mathrm{Th}\right)$ sediment bioturbation intensity along an organic-carbon gradient: Journal of Marine Research, v. 54, p. 763-792.

Wiberg, P.L., and Harris, C.K., 2002, Desorption of p,p'-DDE from sediment during resuspension events on the Palos Verdes shelf, California-a modeling approach: Continental Shelf Research, v. 22, p. 1005-1023.

Wiegel, J., and Wu, Q., 2000, Microbial reductive dehalogenation of polychlorinated biphenyls: FEMS Microbiology Ecology, v. 32, p. 1-15.

Wolfe, N.L., Zepp, R.G., Paris, D.F., Baughman, G.L., and Hollis, R.C., 1977, Methoxychlor and DDT degradation in water-Rates and products: Environmental Science \& Technology, v. 11, no. 12, p. 1077-1081.

Young, D.R., Gossett, R.W., and Heesen, T.C., 1988, Persistence of chlorinated hydrocarbon contamination in a California marine ecosystem: in Wolfe, D.A., and O'Connor, T.P., eds., Oceanic Processes in Marine Pollution-Urban Wastes in Coastal Marine environments, Vol. 5: Malabar, FL, Krieger Publishing Co., p. 33-41.

Zeng, E.Y., and Yu, C.C., 1996, Measurements of linear alkylbenzenes by GC/MS with interference from tetrapropylene-based alkylbenzenes-Calculation of quantitation errors using a two-component model: Environmental Science \& Technology, v. 30, no. 1, p. 322-328.

Zeng, E.Y., and Venkatesan, M.I., 1999, Dispersion of sediment DDTs in the coastal ocean off southern California: The Science of the Total Environment, v. 229, p. 195-208.

Zeng, E.Y., Cheng, D., Khan, A.R., and Vista, C.L., 1998, Validity of using linear alkylbenzenes as markers of sewage contamination with interference from tetrapropylene-based alkylbenzenes: Environmental Toxicology and Chemistry, v. 17, no. 3, p. 394-397.

Zepp, R.G., Wolfe, N.L., Azarraga, L.V., Cox, R.H., and Pape, C.W., 1977, Photochemical transformation of the DDT and methoxychlor degradation products, DDE and DMDE, by sunlight: Archives of Environmental Contamination and Toxicology, v. 6, p. 305-314.

\section{Appendices}

1. Procedures for Preparation of Clean Reagents and Labware: Trace Organics

2. Fractionation of Sediment Extracts for Determination of Trace Organics

3. Sectioning of Sediment Cores

4. Extraction of Sediments for Determination of Trace Organics

5. Instrumental Analysis for the Long-Chain Alkylbenzenes

6. Instrumental Analysis for Total Organic Carbon and Total Nitrogen in Sediments

7. Instrumental Analysis for Chlorinated Hydrocarbons 


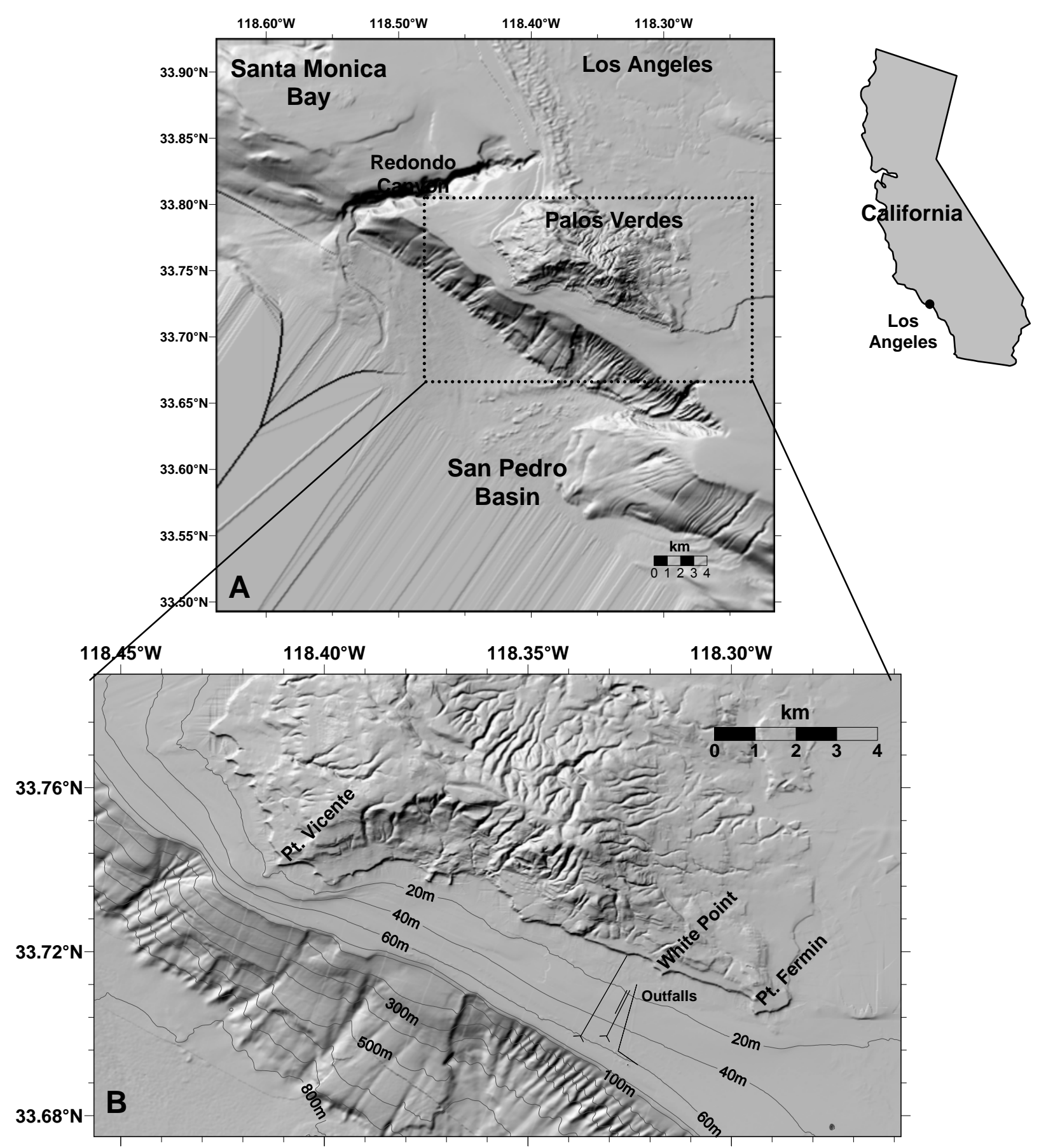

Figure 1. Shaded-relief maps showing $(A)$ Los Angeles, the Palos Verdes Peninsula, and adjoining coastal bathymetry, and $(B)$ the Palos Verdes Shelf study site. Contours are in meters below sea level. Depths from Peter Dartnell (USGS, written commun., 2007) were used by the first author to make these maps. The bathymetry has a horizontal datum of the World Geodetic System of 1984 (WGS 84) and a vertical datum of Mean Lower Low Water. The topography has a horizontal datum of WGS 84 (converted from the North American Datum of 1983, NAD 83) and a vertical datum of the North American Vertical Datum of 1988 (NAVD 88). 
$\%$ DDMU

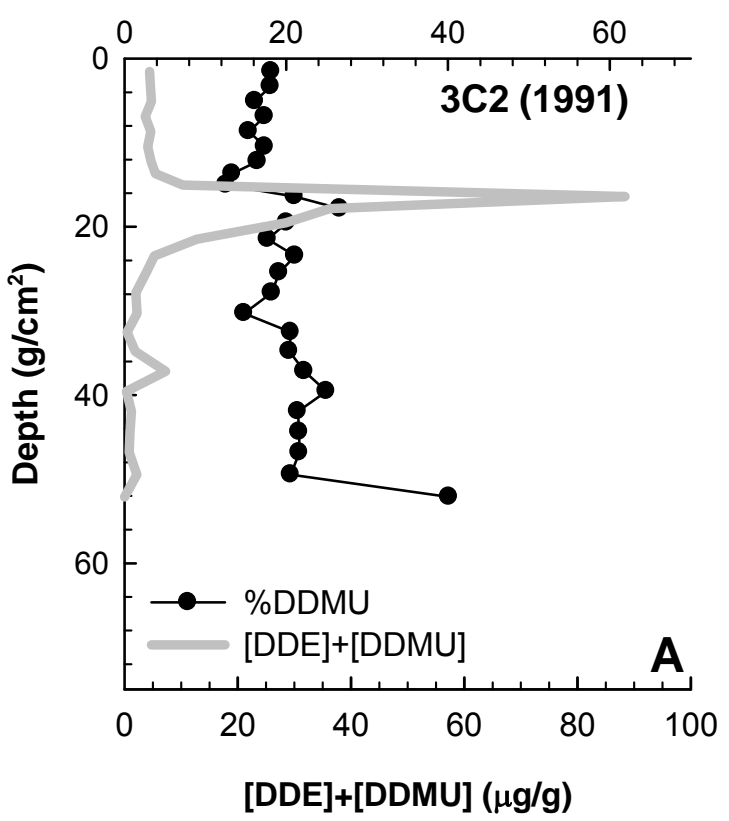

$\%$ DDMU

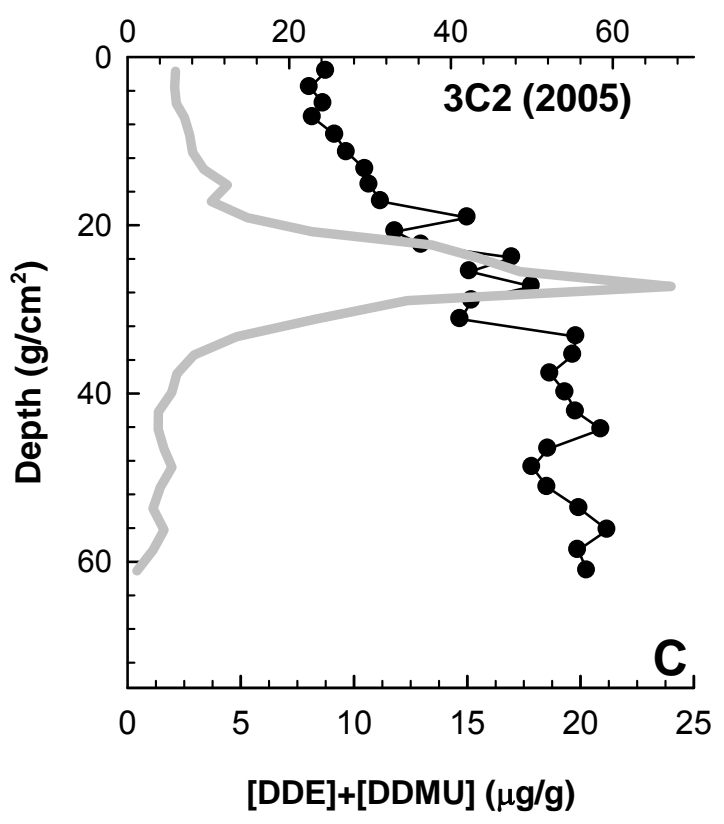

\% DDMU

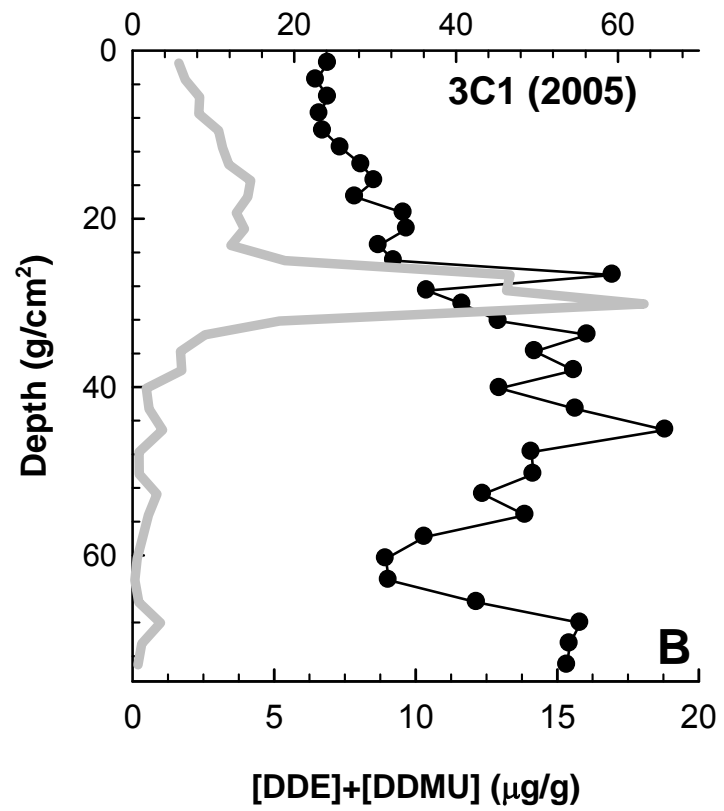

\% DDMU

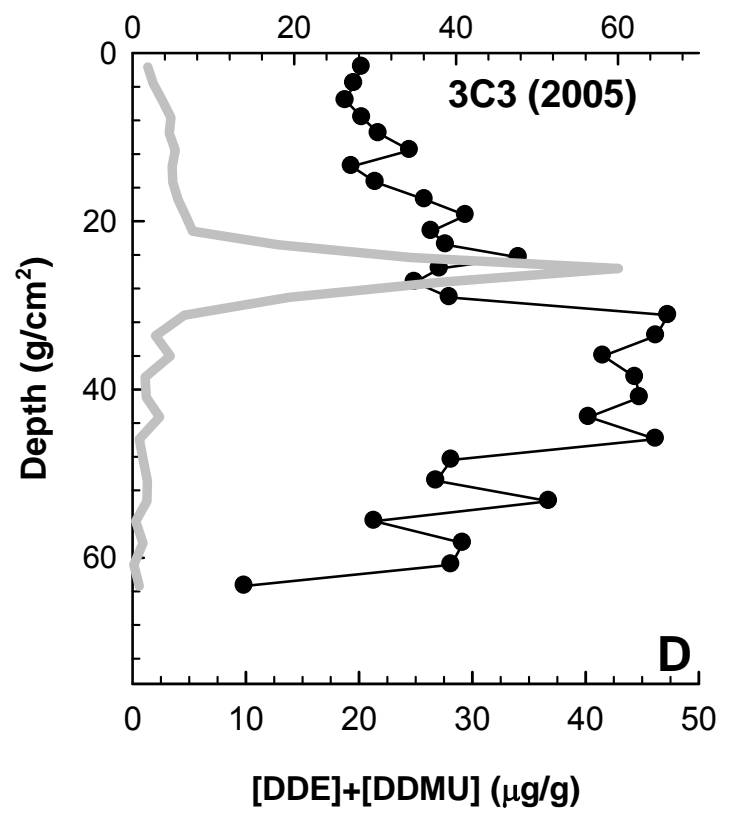

Figure 2. Graphs showing percent $p, p^{\prime}$-DDMU (relative to the sum, $p, p^{\prime}$-DDMU $+p, p^{\prime}$-DDE) and vertical concentration $\left(p, p^{\prime}\right.$-DDMU $+p, p^{\prime}$-DDE) profiles in cores collected by the LACSD (Los Angeles County Sanitation Districts) at station 3C in 1991 and 2005. See table 2 for station locations for these cores. 
\% DDMU

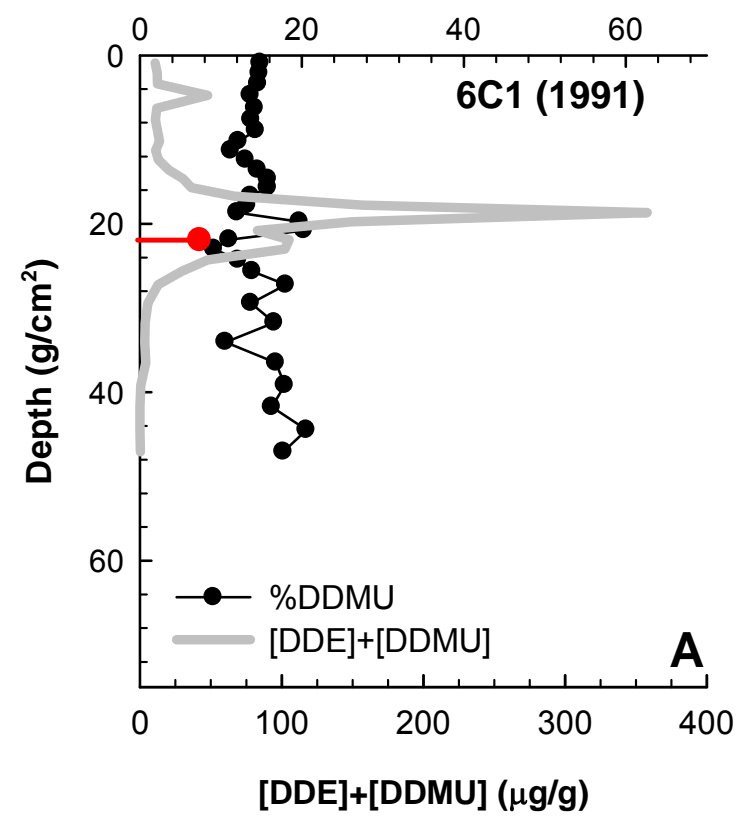

$\%$ DDMU

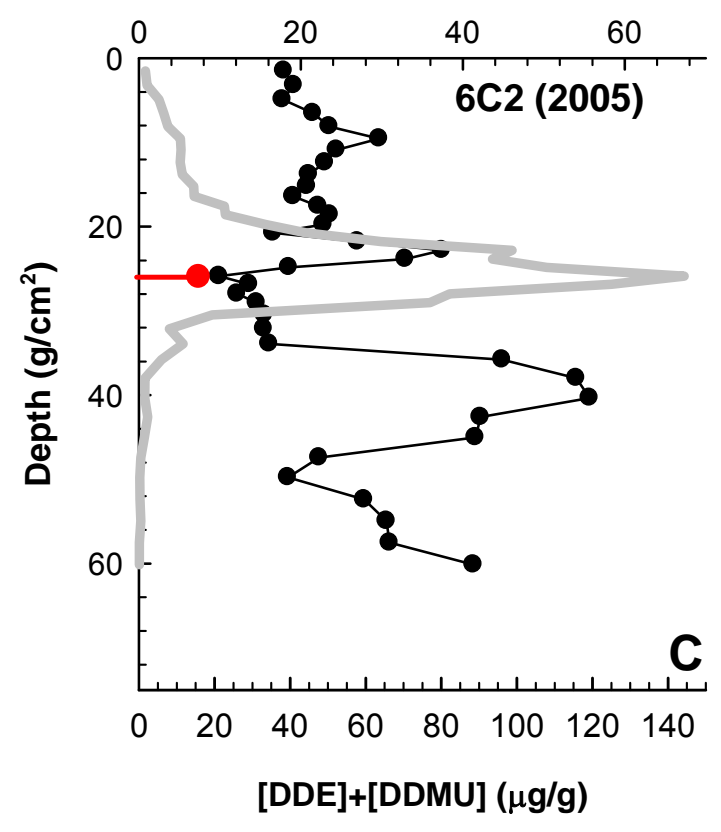

\% DDMU

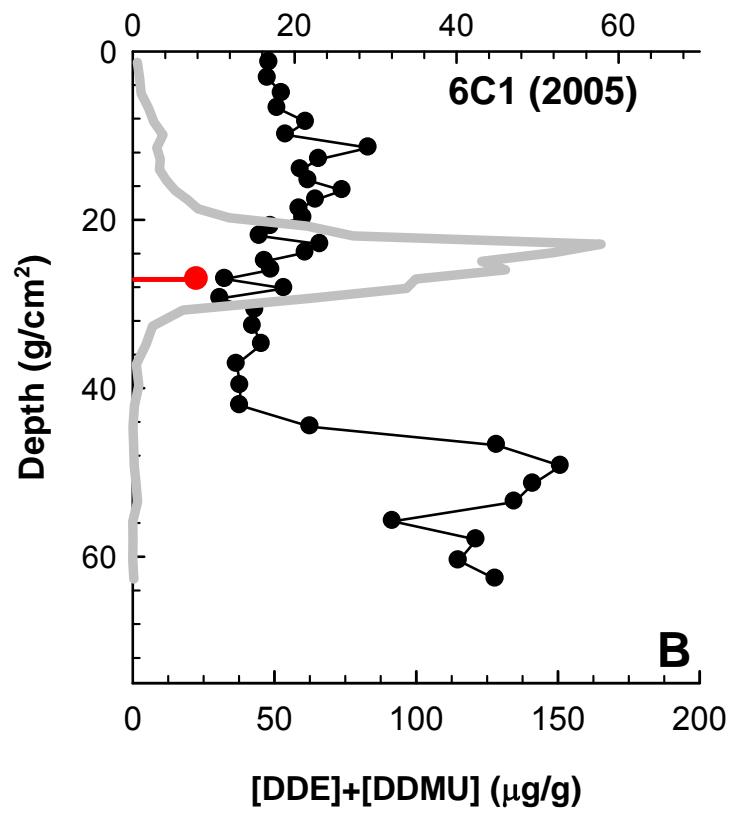

\% DDMU

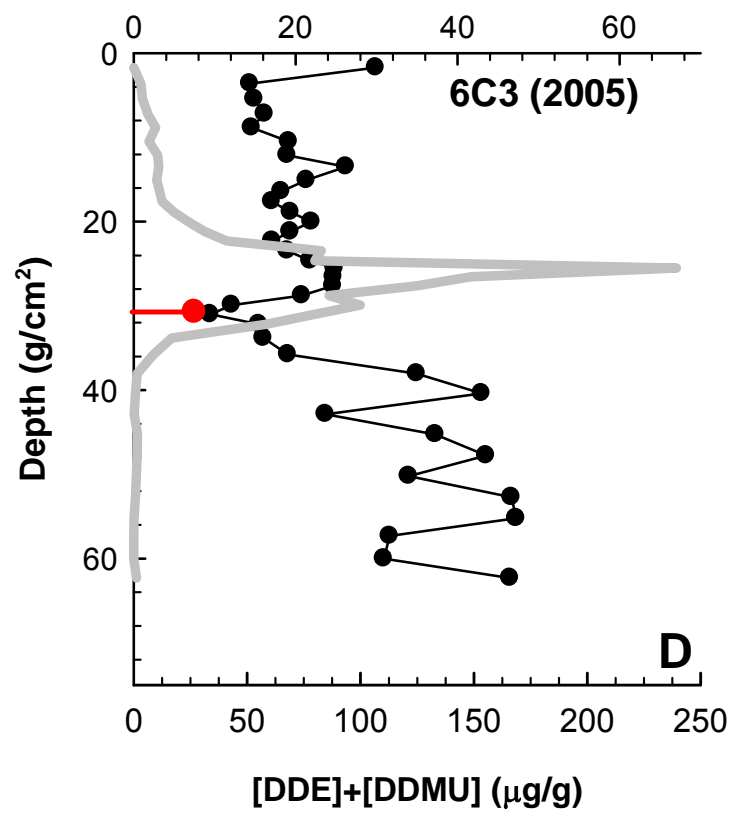

Figure 3. Graphs showing percent $p, p^{\prime}$-DDMU (relative to the sum, $p, p^{\prime}$-DDMU $+p, p^{\prime}$-DDE) and vertical concentration $\left(p, p^{\prime}\right.$-DDMU $+p, p^{\prime}$-DDE) profiles in cores collected by the LACSD (Los Angeles County Sanitation Districts) at station 6C in 1991 and 2005. See table 2 for station locations for these cores. Red bars indicate reduced $p, p^{\prime}$-DDMU abundance. 

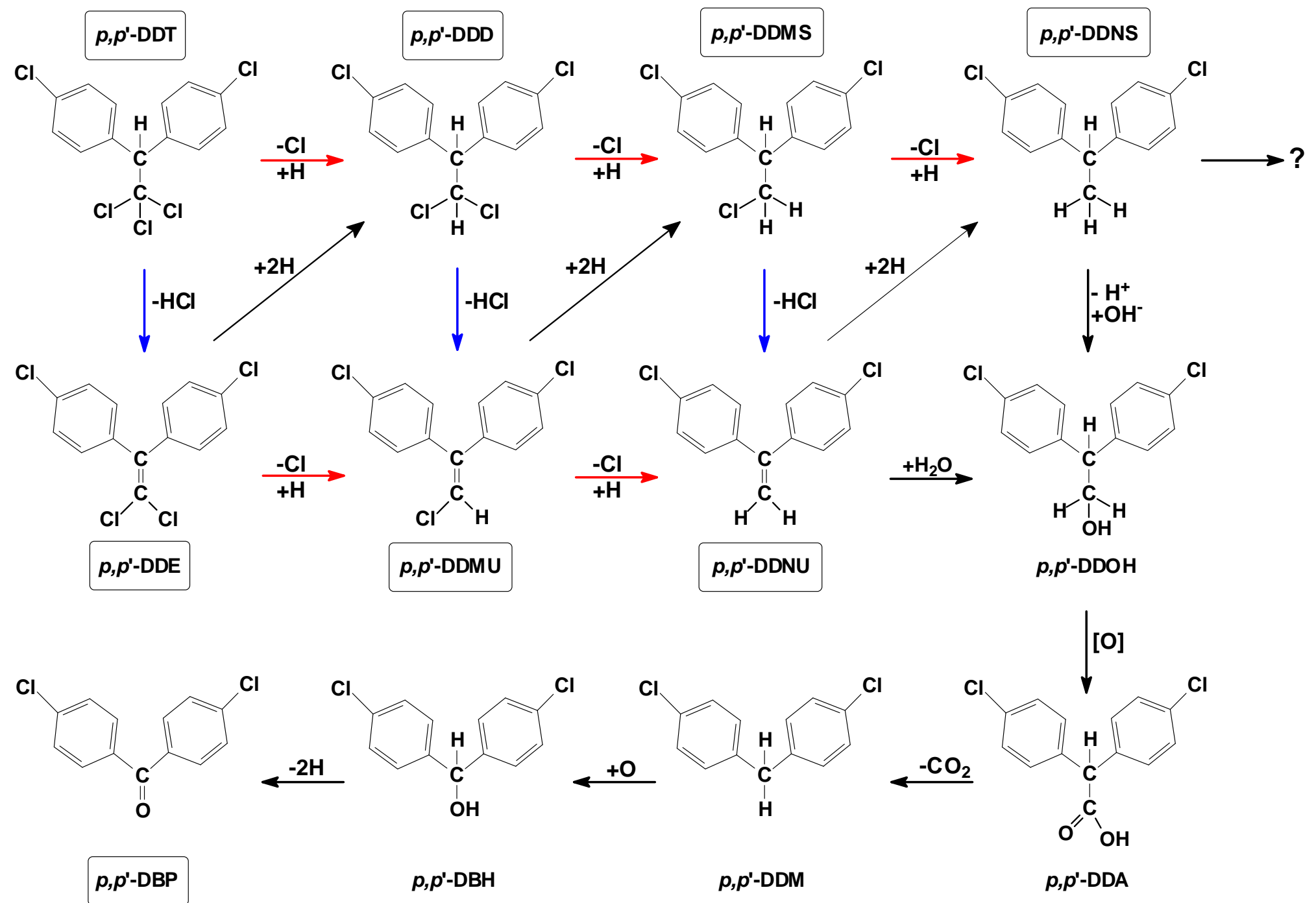

Figure 4. Chart showing potential pathways for microbial degradation of DDT. Blue arrows represent dehydrochlorination reactions; red arrows represent reductive dechlorination reactions. Compounds with names enclosed in boxes have been detected in Palos Verdes Shelf sediments. (Adapted from Lal and Saxena, 1982; Rochkind-Dubinsky and others,1987; Aislabie and others, 1997. 

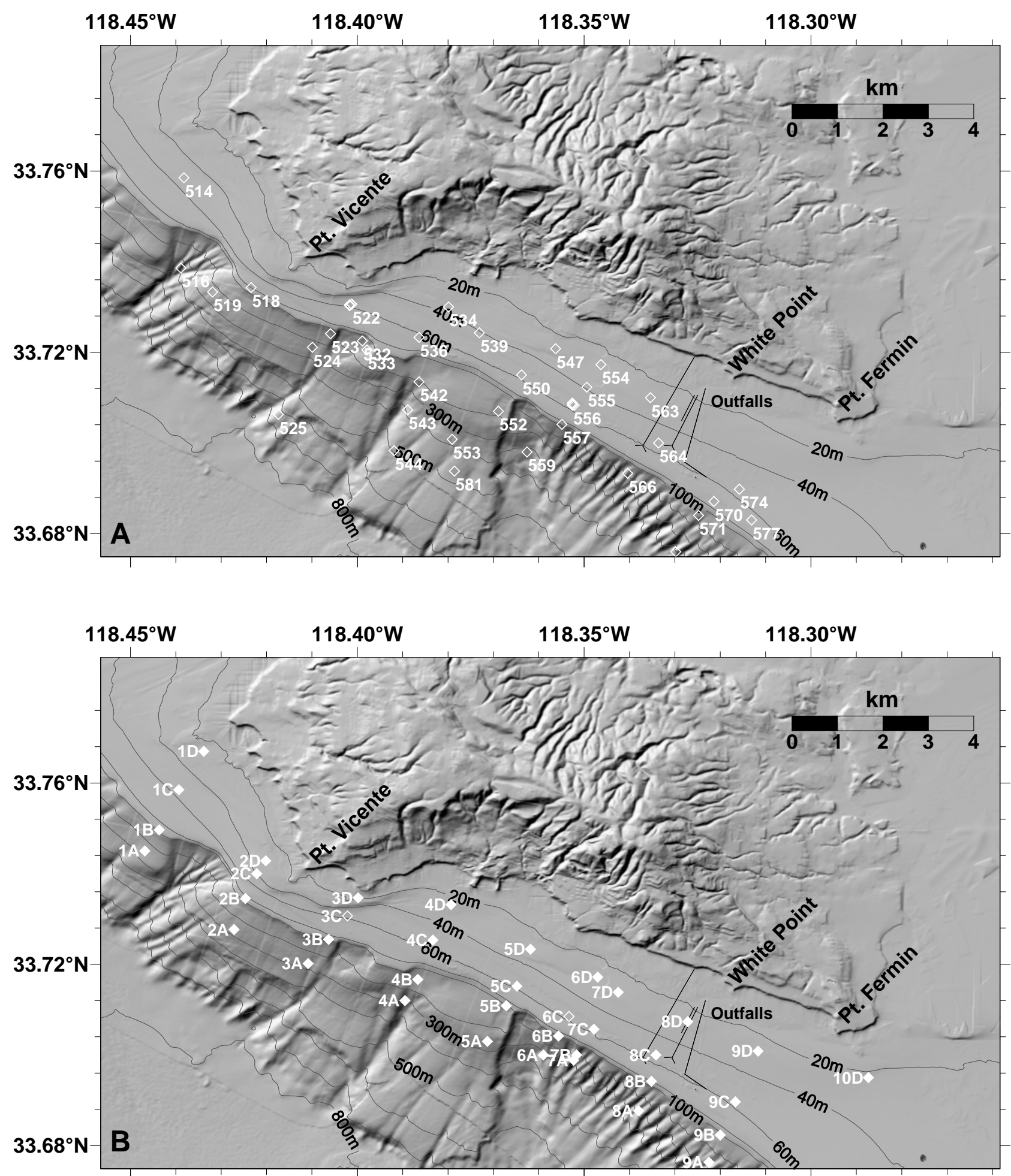

Figure 5. Shaded relief maps showing sediment coring stations: (A) USGS-1992, (B) LACSD (Los Angeles County Sanitation Districts), (C) and (D) SAIC (Science Applications International Corporation) 2004 (SU is a cell designation). Bathymetric contours are in meters below sea level. 


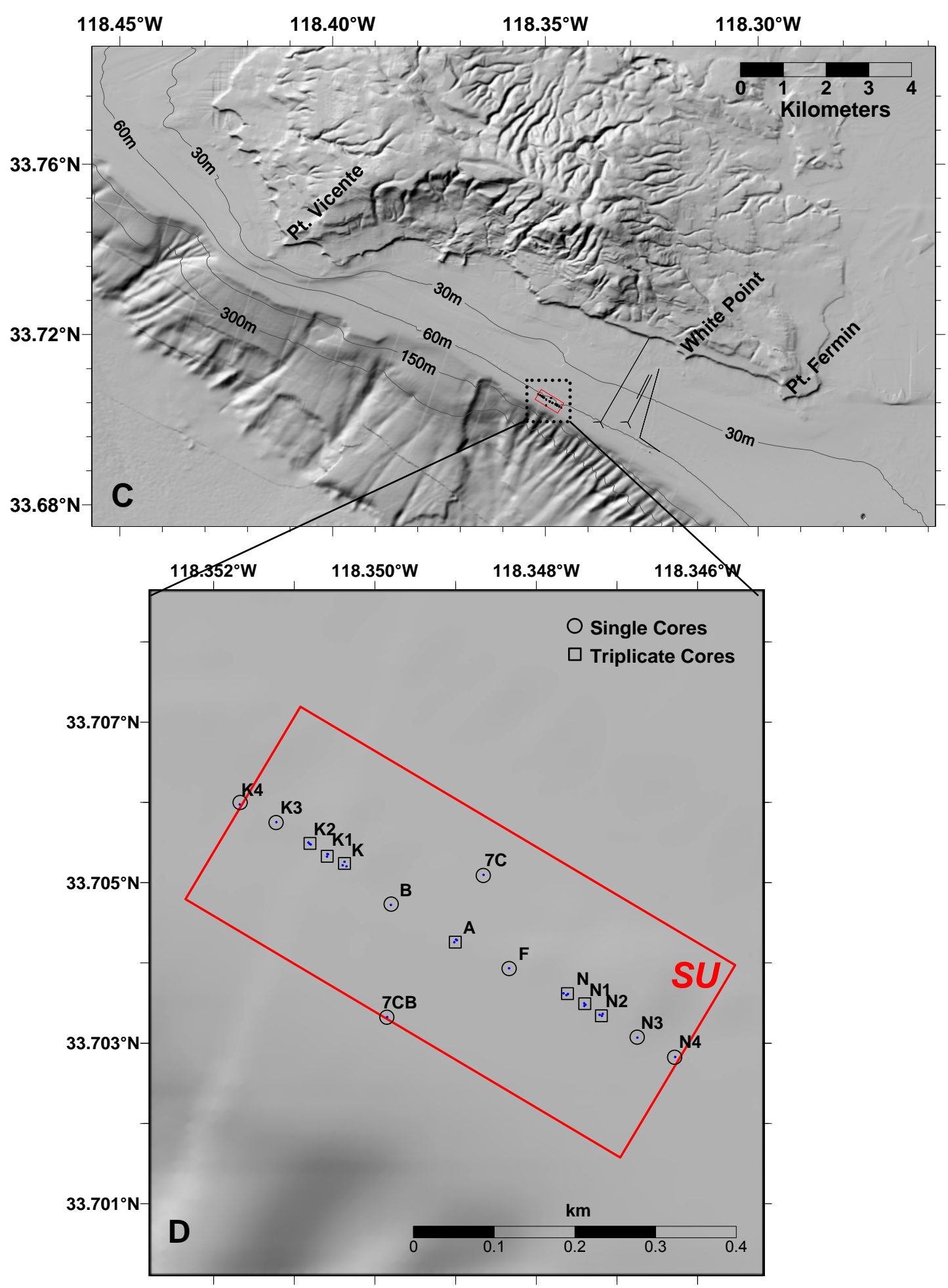

Figure 5 - continued. Shaded relief maps showing sediment coring stations: (A) USGS-1992, (B) LACSD (Los Angeles County Sanitation Districts), (C) and (D) SAIC (Science Applications International Corporation) 2004 (SU is a cell designation). Bathymetric contours are in meters below sea level. 


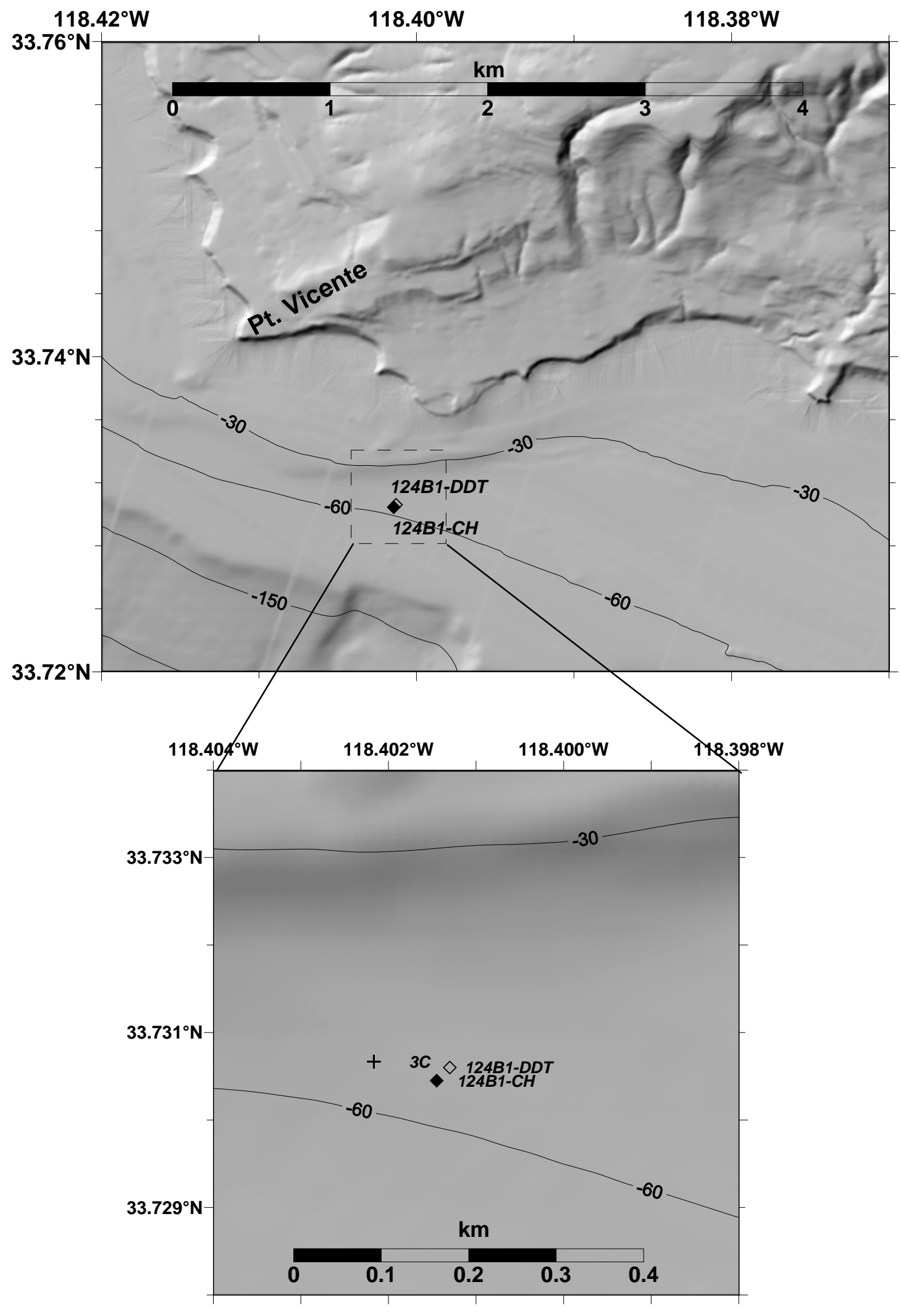

Figure 6. Shaded relief maps showing locations of cores collected by the USGS in 1992 and 2003. Bathymetric contours are in meters below sea level. 


\section{Sediment Extruder}
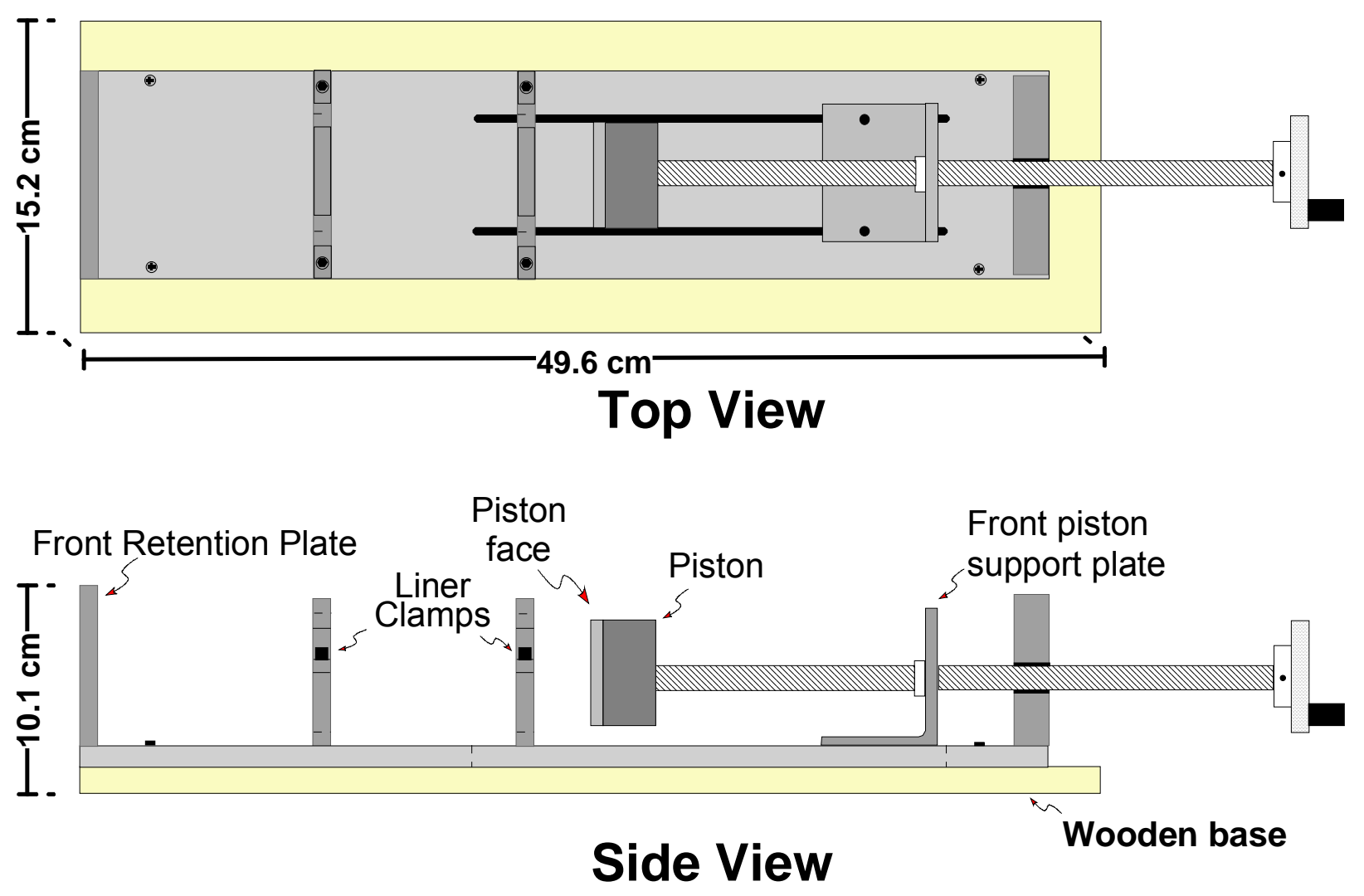

Figure 7. Diagram showing extruder used for sectioning frozen sediment cores. 

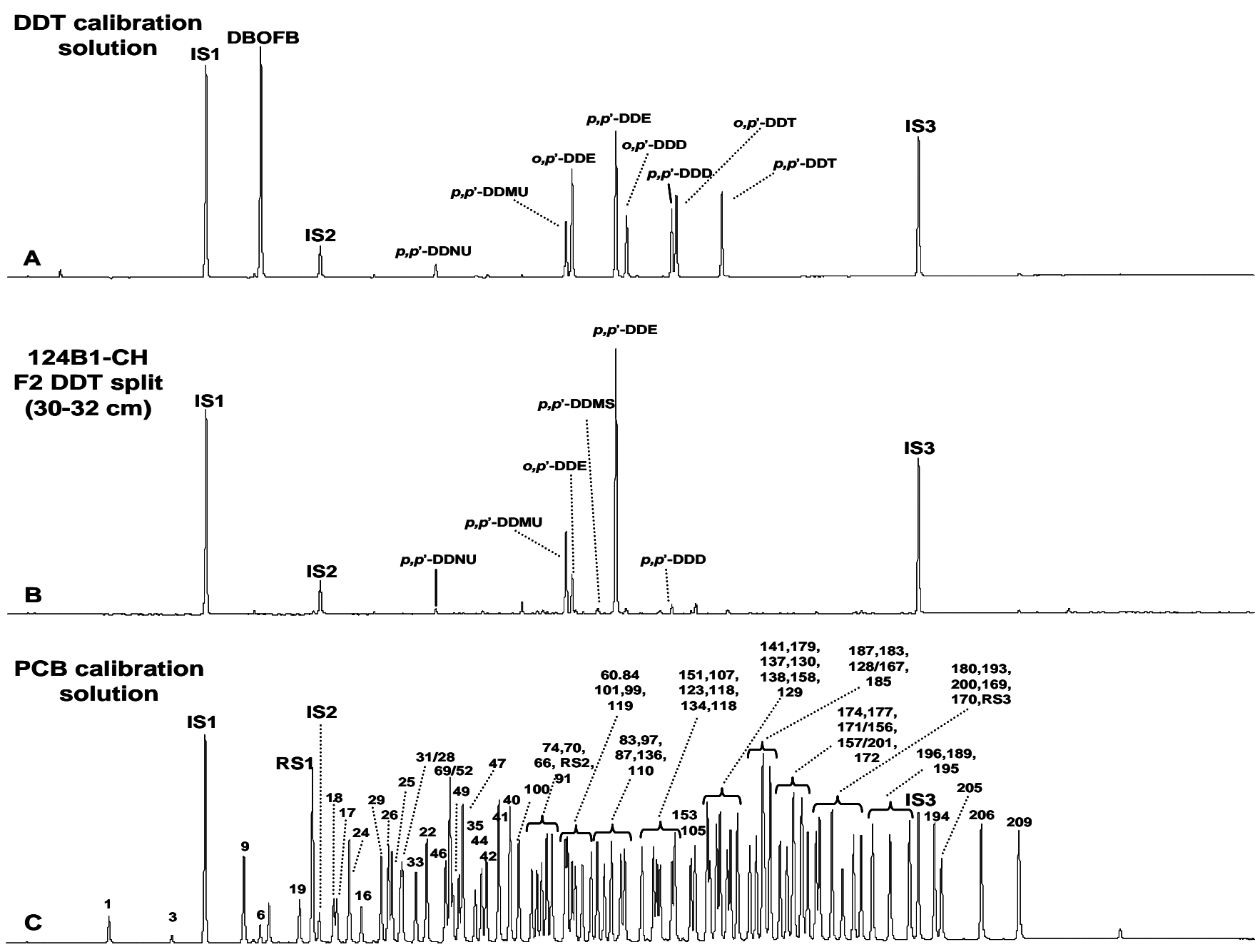

124B1-CH F2 PCB split $(30-32 \mathrm{~cm})$

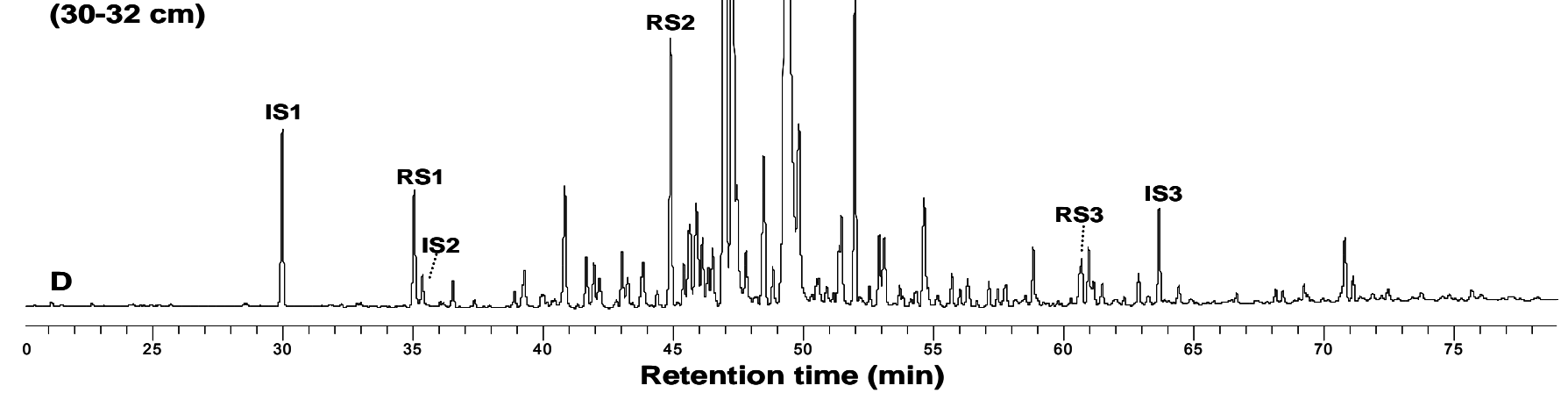

Figure 8. Figure showing gas chromatograms of $(A)$ DDT calibration standard solution, $(B)$ DDT F2 split of 30-32 cm section of sediment core 124B1-CH (2003), (C) PCB (polychorinated biphenyl) calibration standard solution, and (D) PCB F2 split of 30-32 cm section of sediment core 124B1-CH (2003). PCB congener labels use the IUPAC (International Union of Pure and Applied Chemistry) naming convention. Note: IS1=tetrachloro-meta-xylene (TCMX), IS2=PCB11, IS3=PCB207, RS1=PCB30, RS2=PCB121, RS3=PCB198. 

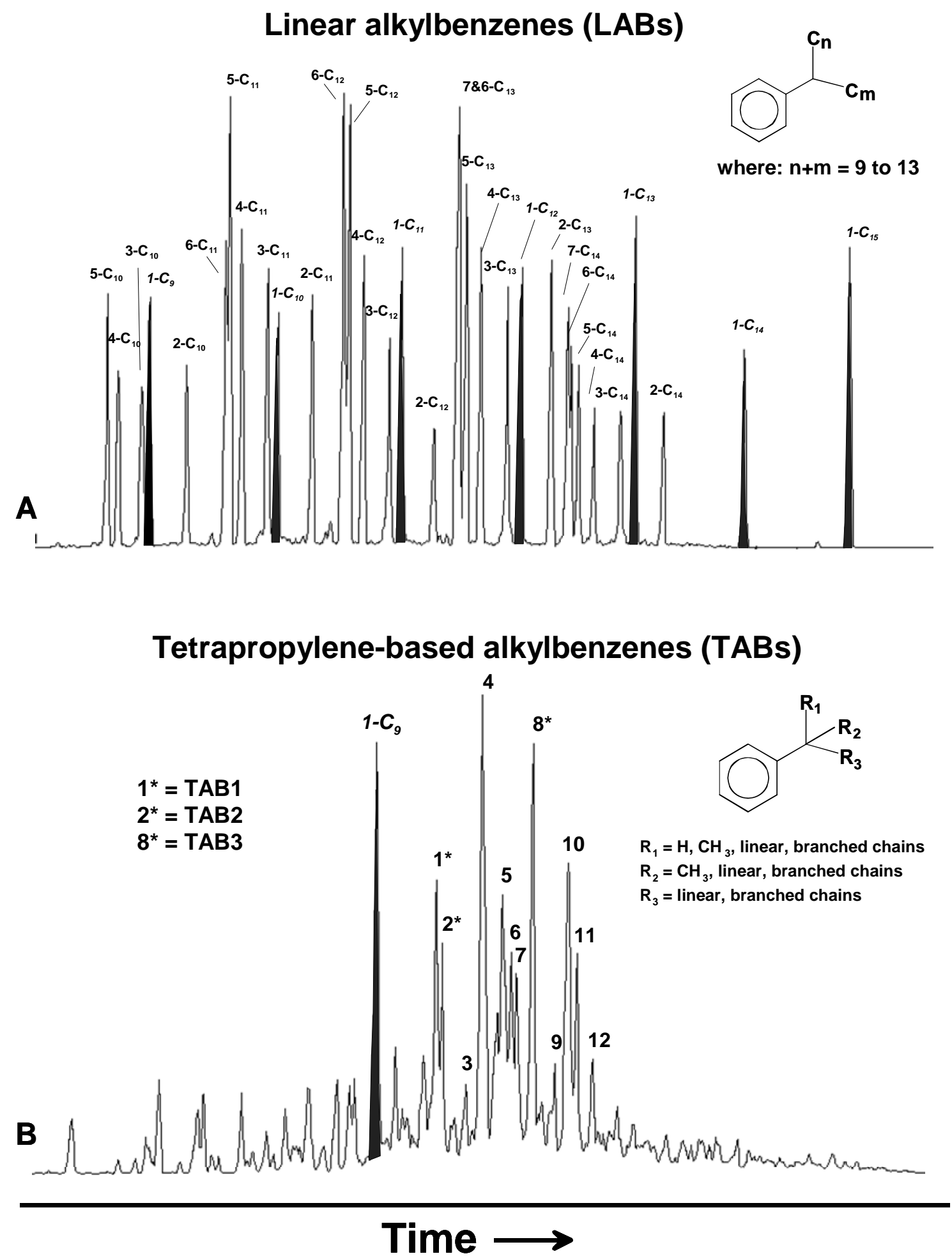

Figure 9. Figure showing gas chromatograms and structures of $(A)$ linear alkylbenzenes (LABs), and $(B)$ tetrapropylene-based alkylbenzenes (TABs). Chromatogram $x$-axes are not aligned. Numbers above $T A B$ peaks indicate TABs quantitated in this study. TAB1, TAB2, and TAB3 are designations as given in Eganhouse and others (2000). Shaded peaks are internal quantitation standards or surrogates. 


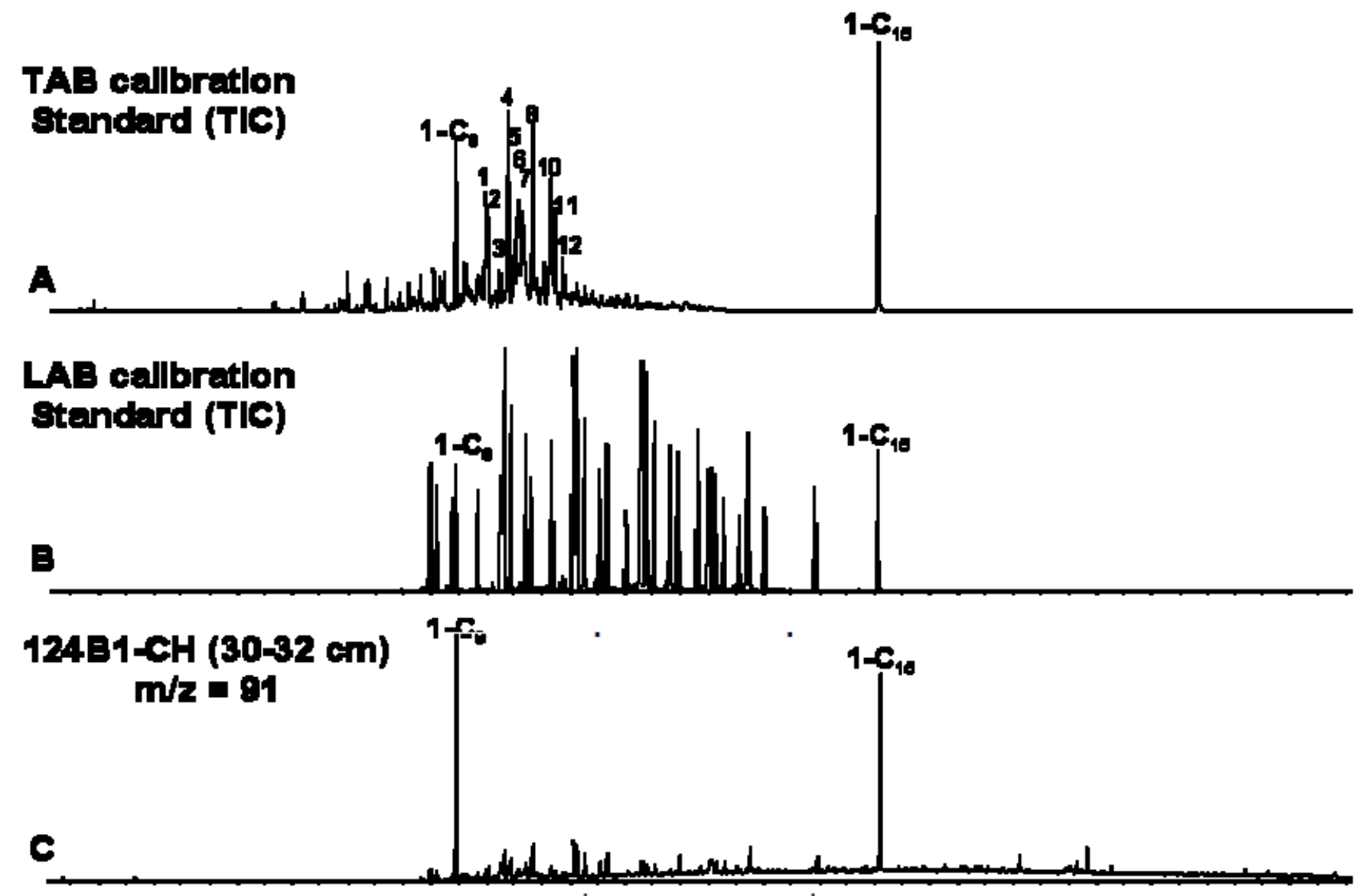

$124 \mathrm{B1}-\mathrm{CH}(30-32 \mathrm{~cm})$

$m / 2=105$

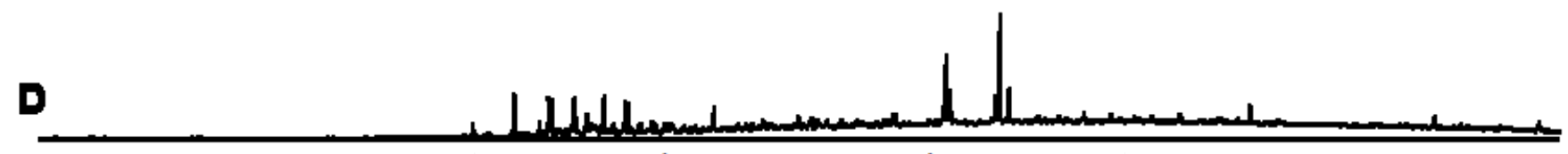

\section{$124 \mathrm{B1}-\mathrm{CH}(30-32 \mathrm{~cm})$ $\mathrm{m} / \mathbf{z}=118$}

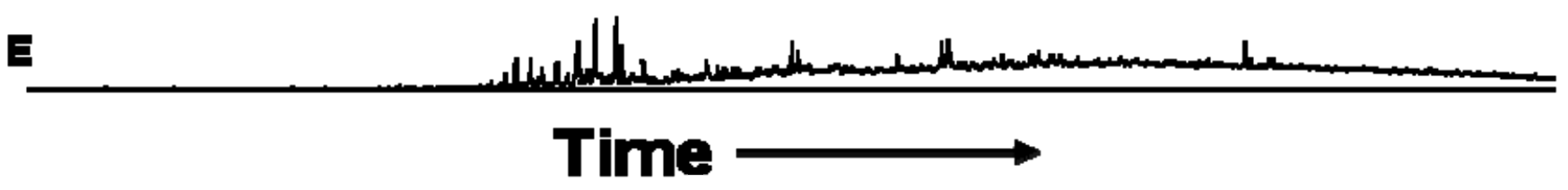

Figure 10. Figure showing total ion current (TIC) of $(A)$ tetrapropylene-based alkylbenzene (TAB) calibration standard, and $(B)$ linear alkylbenzene (LAB) calibration standard, and mass fragmentograms for LCAB (long-chain alkylbenzene) F2 split of 30-32 cm section of 124B1-CH (2003) at $(C) \mathrm{m} / \mathrm{z}=91,(D) \mathrm{m} / \mathrm{z}$ $=105,(E) \mathrm{m} / \mathrm{z}=119(\mathrm{~m} / \mathrm{z}=\mathrm{mass} /$ charge ratio $)$. 


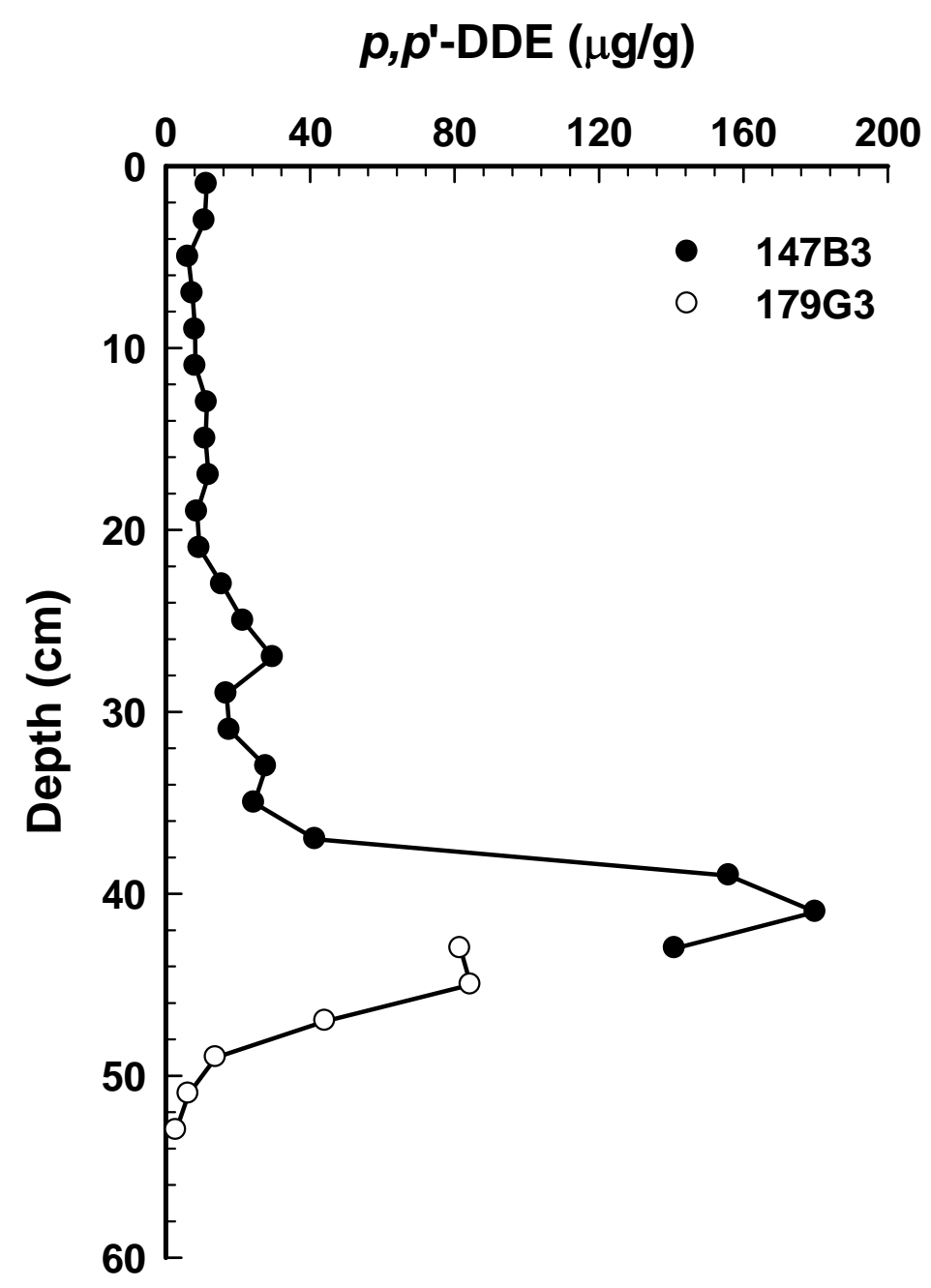

Figure 11. Graph showing vertical concentration profiles of $p, p^{\prime}$-DDE in box core 147B3 (filled circles) and gravity core 179G3 (open circles) collected in 1992 at station 556 by the USGS (Lee, 1994a; Bailey and Costa, 1994). See figure 5A for station location. Depth (y-axis) is given in centimeters below the sediment-water interface. 


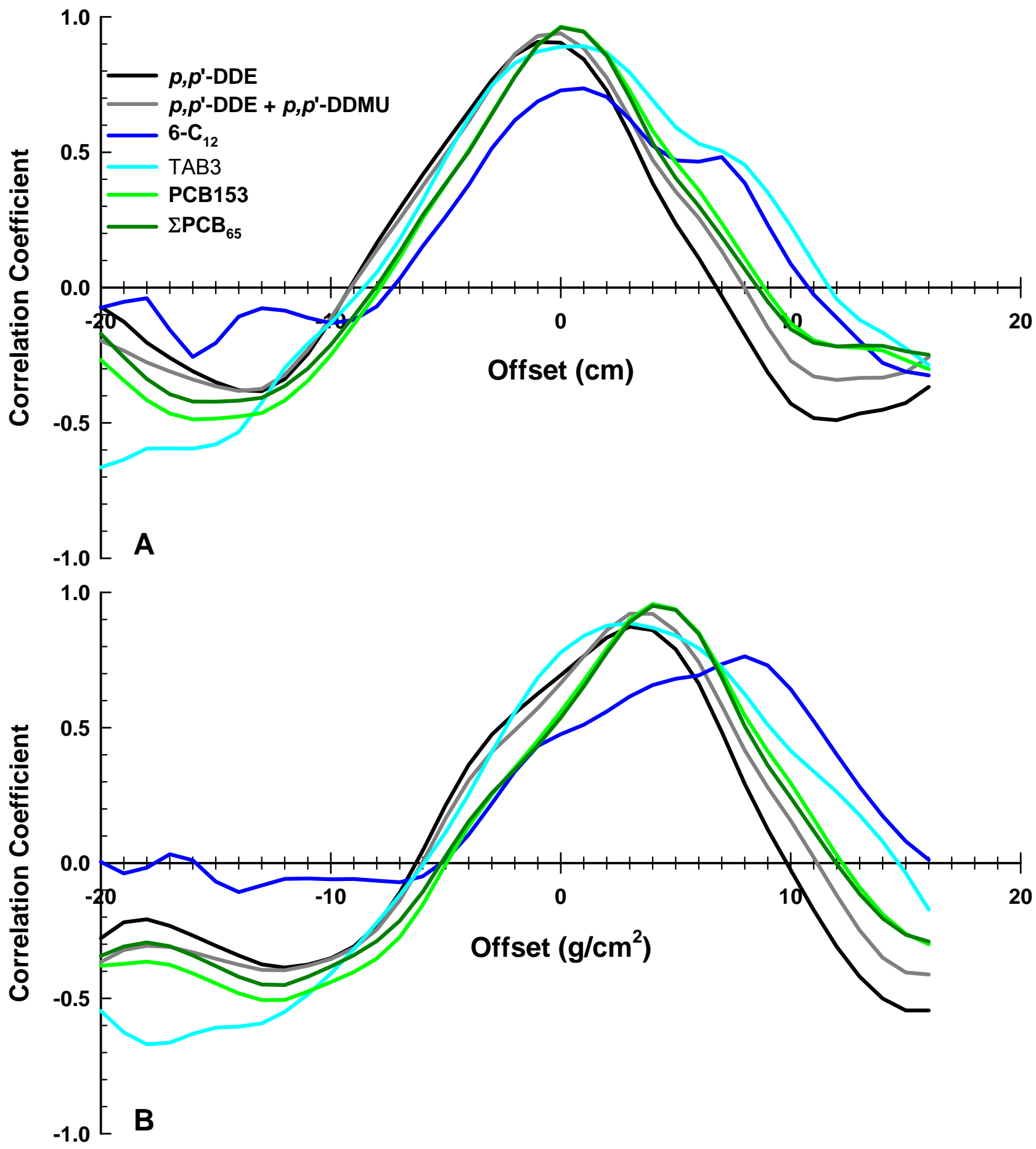

Figure 12. Graphs showing core alignment curves based on Pearson correlation analyses of concentration/inventory compared to $(A)$ depth $(\mathrm{cm})$, and $(B)$ mass accumulation $\left(\mathrm{g} / \mathrm{cm}^{2}\right)$ offsets for cores 124B1-DDT (1992) and 124B1-CH (2003). 

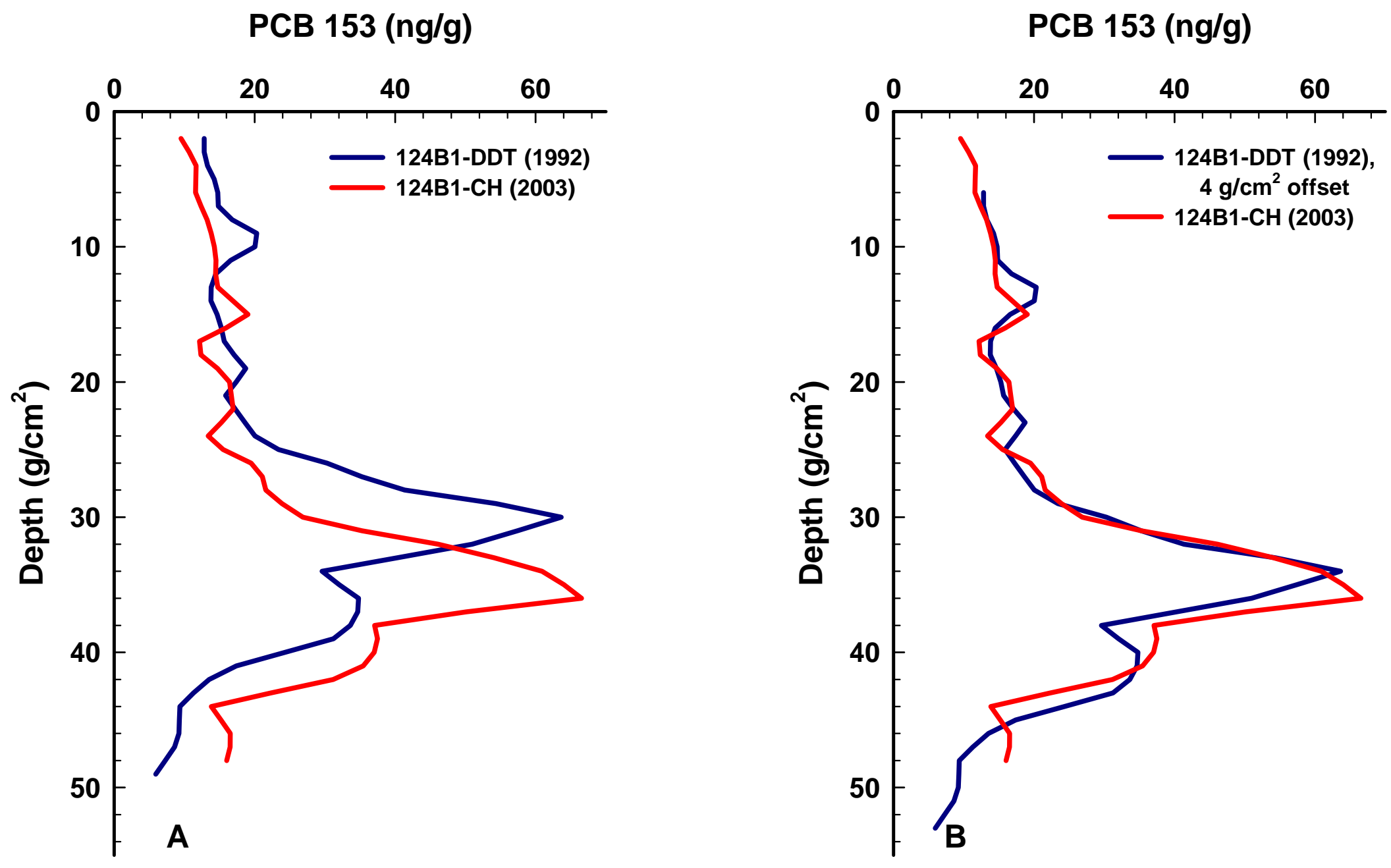

Figure 13. Graphs showing vertical concentration profiles of PCB $153(\mathrm{ng} / \mathrm{g})$ in cores 124B1-DDT (1992-blue lines) and 124B1-CH (2003red lines) cores $(A)$ without depth offset, and $(B)$ with depth offset of $4 \mathrm{~g} / \mathrm{cm}^{2}$. Data interpolated at $1 \mathrm{~g} / \mathrm{cm}^{2}$ were used for these plots. 

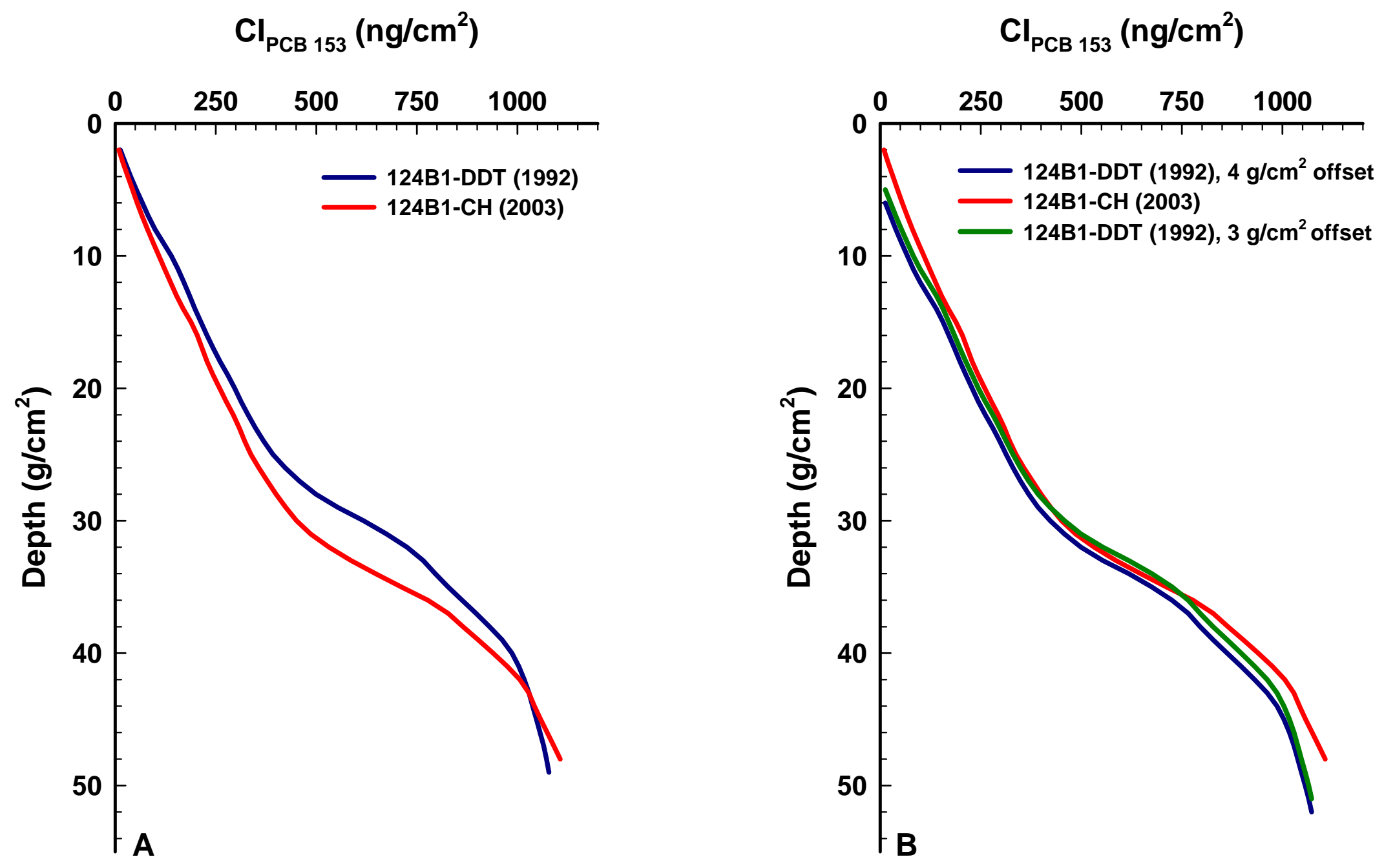

Figure 14. Graphs showing cumulative inventory (CI) profiles for PCB 153 in cores 124B1-DDT (1992) and 124B1-CH (2003): (A) without depth offset, and $(B)$ with depth offsets of 3 and $4 \mathrm{~g} / \mathrm{cm}^{2}$. Data interpolated at $1 \mathrm{~g} / \mathrm{cm}^{2}$ were used for these plots. 

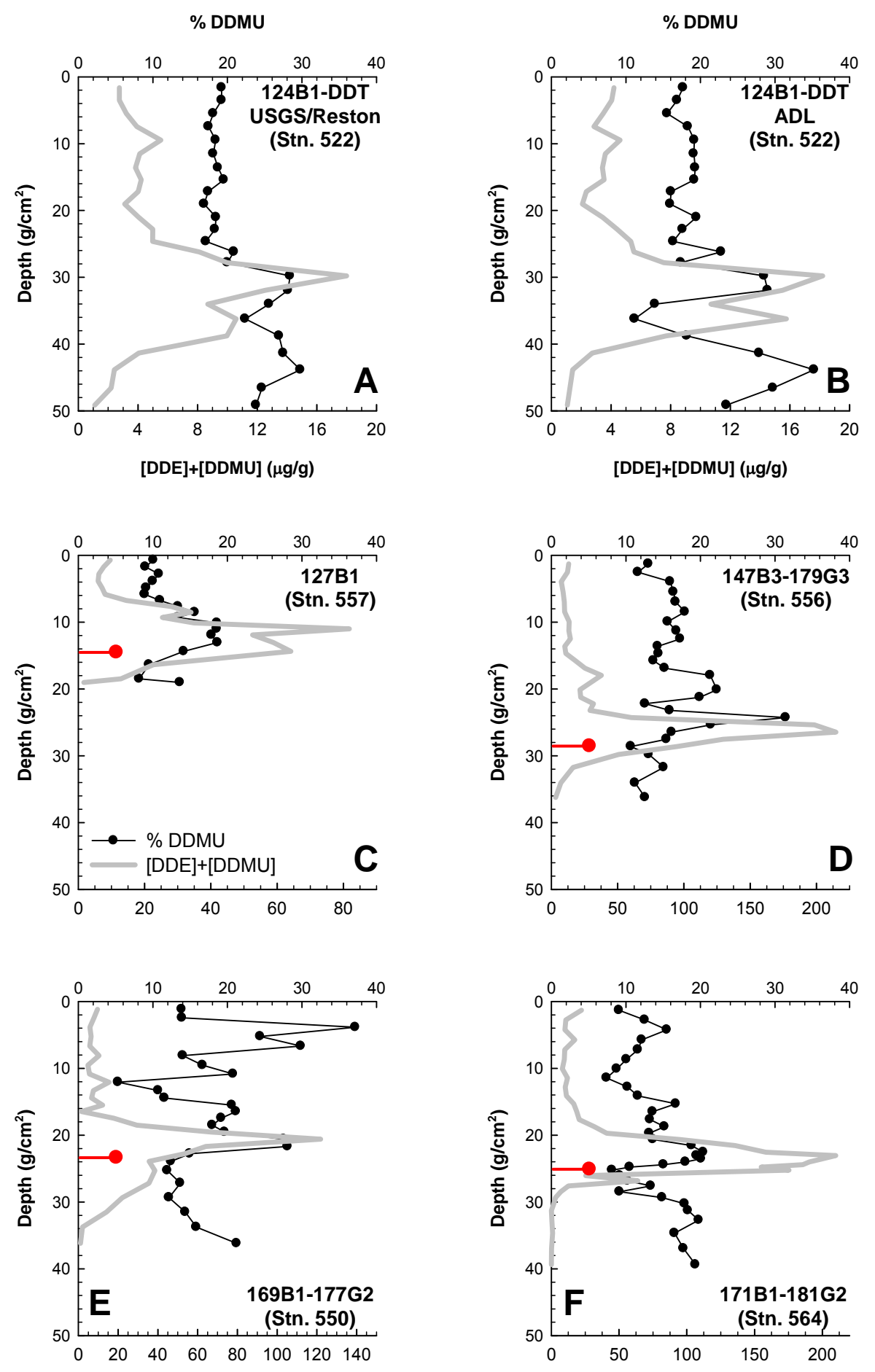

Figure 15. Graphs showing percent $p, p^{\prime}$-DDMU (relative to the sum, $p, p^{\prime}$-DDMU $+p, p^{\prime}-\mathrm{DDE}$; filled circles) and vertical concentration ( $p, p^{\prime}$-DDMU $+p, p^{\prime}$-DDE) profiles (solid gray lines) for cores collected by the USGS in 1992 (ADL = Arthur D. Little). See figure 5A for station locations. Red bars indicate reduced $p, p^{\prime}$-DDMU abundance. The uncertainty (standard error) of each \% DDMU data point in panel $\mathrm{A}$ is estimated at $16-21 \%$ of its value 


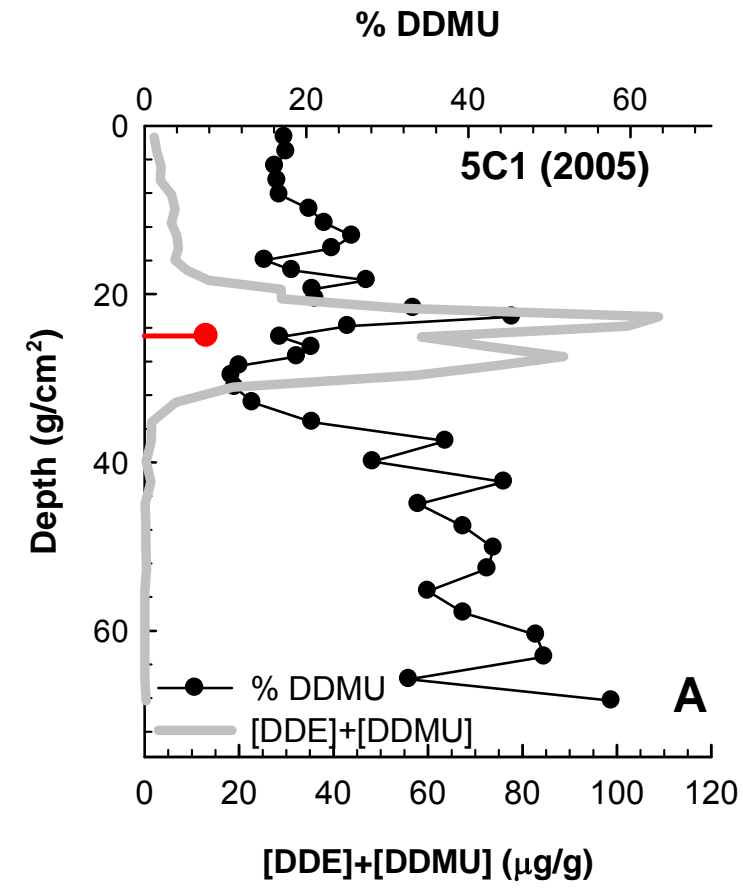

\% DDMU

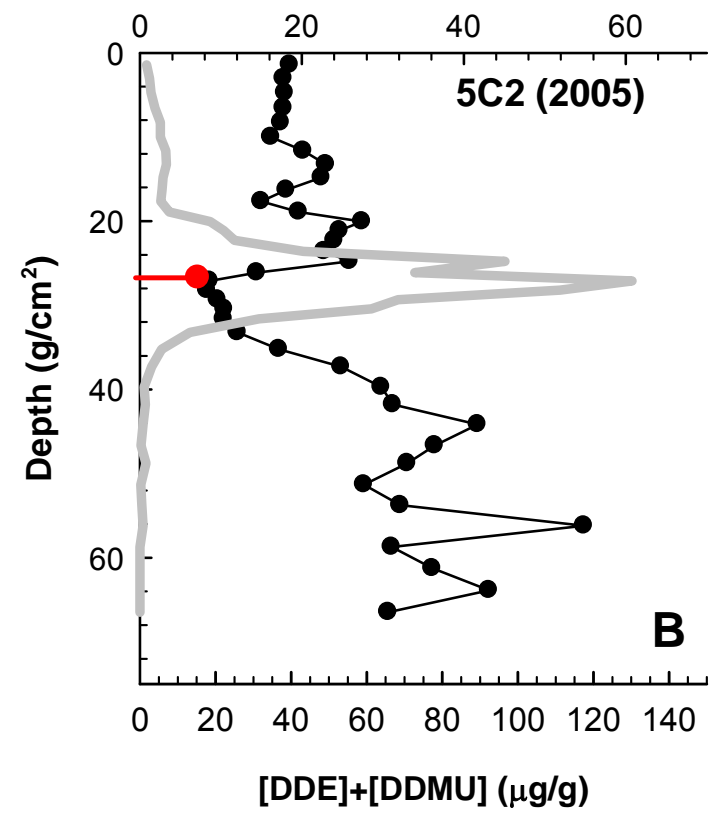

\% DDMU

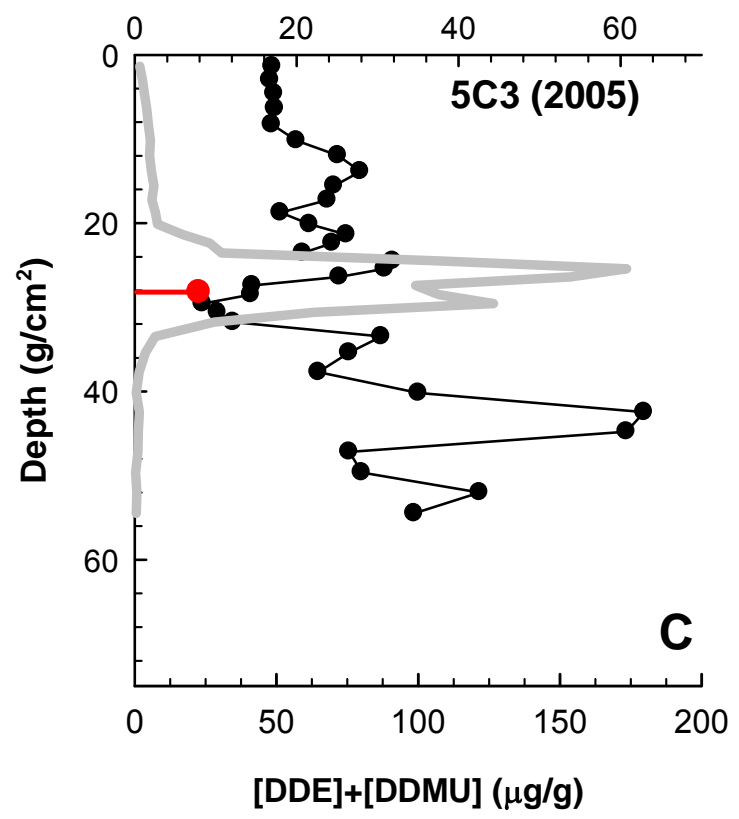

Figure 16. Graphs showing percent $p, p^{\prime}$-DDMU (relative to the sum, $p, p^{\prime}$-DDMU $+p, p^{\prime}$-DDE; filled circles) and vertical concentration ( $p, p^{\prime}$-DDMU $+p, p^{\prime}$-DDE) profiles (solid gray lines) for cores collected by the LACSD (Los Angeles County Sanitation Districts) at station 5C in 2005. See table 2 for station locations of these cores. Red bars indicate reduced $p, p^{\prime}$-DDMU abundance. 

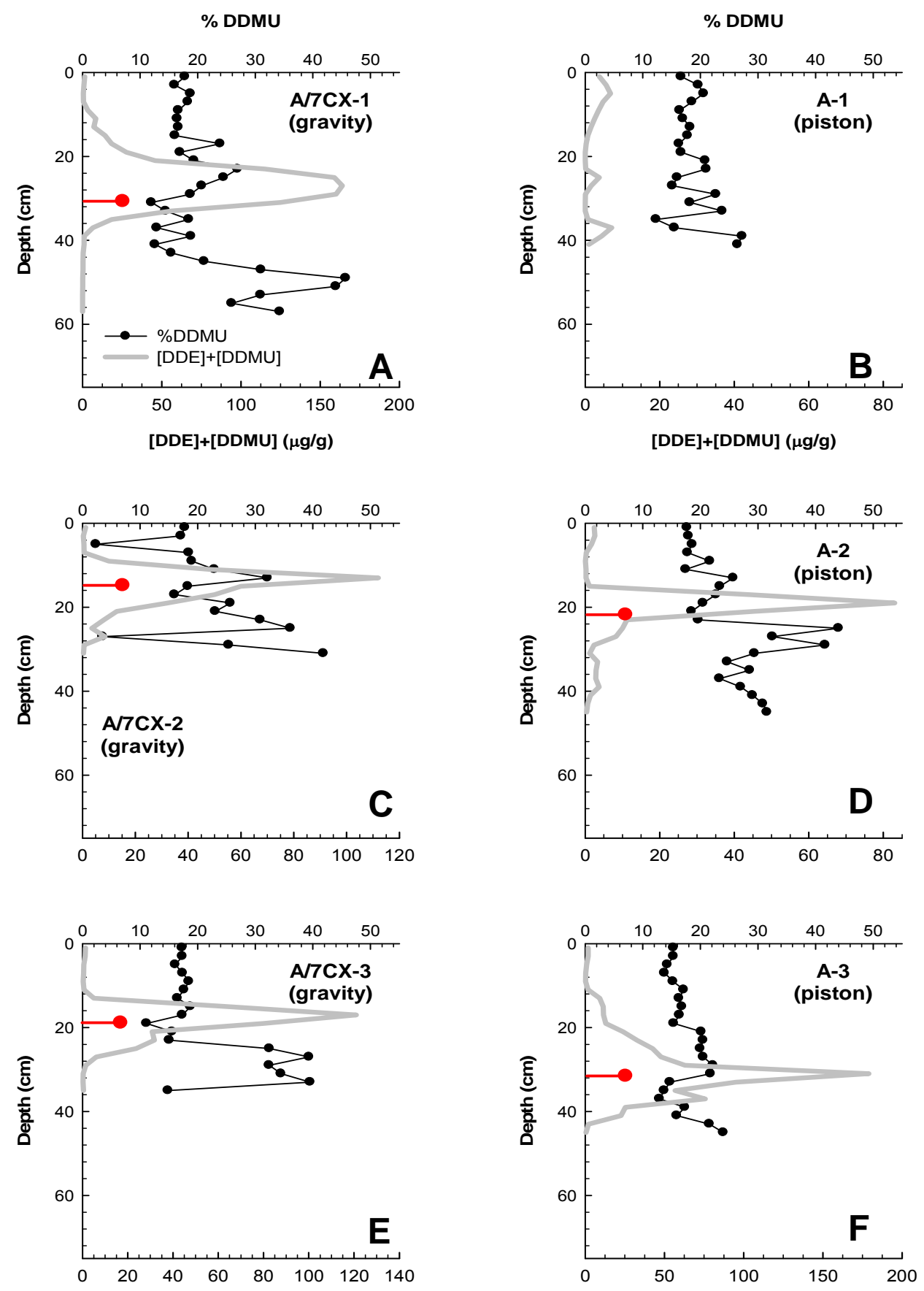

Figure 17. Graphs showing percent $p, p^{\prime}$-DDMU (relative to the sum, $p, p^{\prime}$-DDMU $+p, p^{\prime}$-DDE; filled circles) and vertical concentration ( $p, p^{\prime}$-DDMU $+p, p^{\prime}$-DDE) profiles (solid gray lines) for cores collected by the LACSD (Los Angeles County Sanitation Districts; gravity) and SAIC (Science Applications International Corporation; piston) in 2004 at station A. See table 2 for station locations of these cores. Red bars indicate reduced $p, p^{\prime}$-DDMU abundance. 

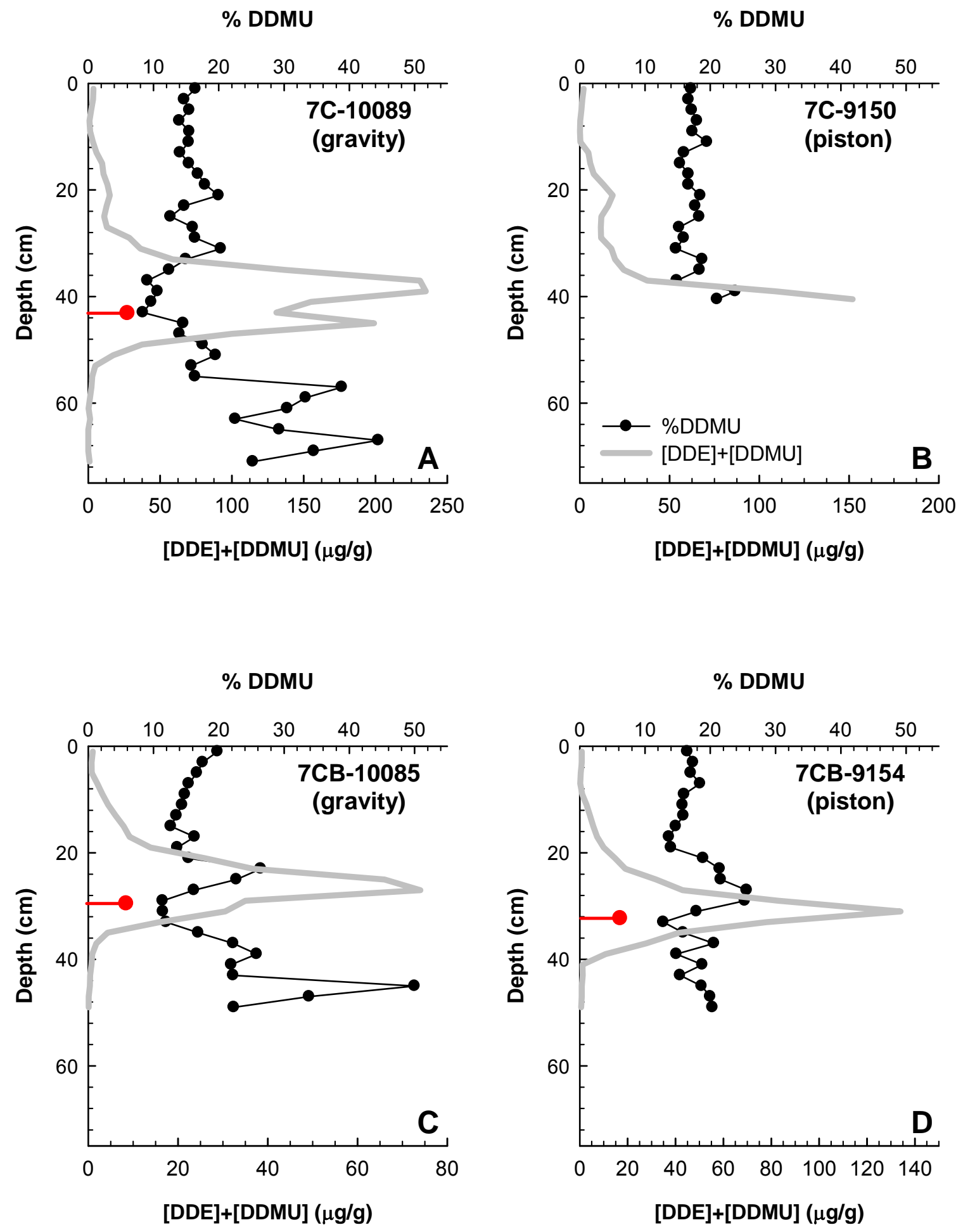

Figure 18. Graphs showing percent $p, p^{\prime}$-DDMU (relative to the sum, $p, p^{\prime}$-DDMU $+p, p^{\prime}$-DDE; filled circles) and vertical concentration ( $p, p^{\prime}$-DDMU $+p, p^{\prime}$-DDE) profiles (solid gray lines) for cores collected by the LACSD (Los Angeles County Sanitation Districts; gravity) and SAIC (Science Applications International Corporation; piston) in 2004 at stations 7 C and 7CB. See table 2 for station locations of these cores. Red bars indicate reduced $p, p^{\prime}$-DDMU abundance. 

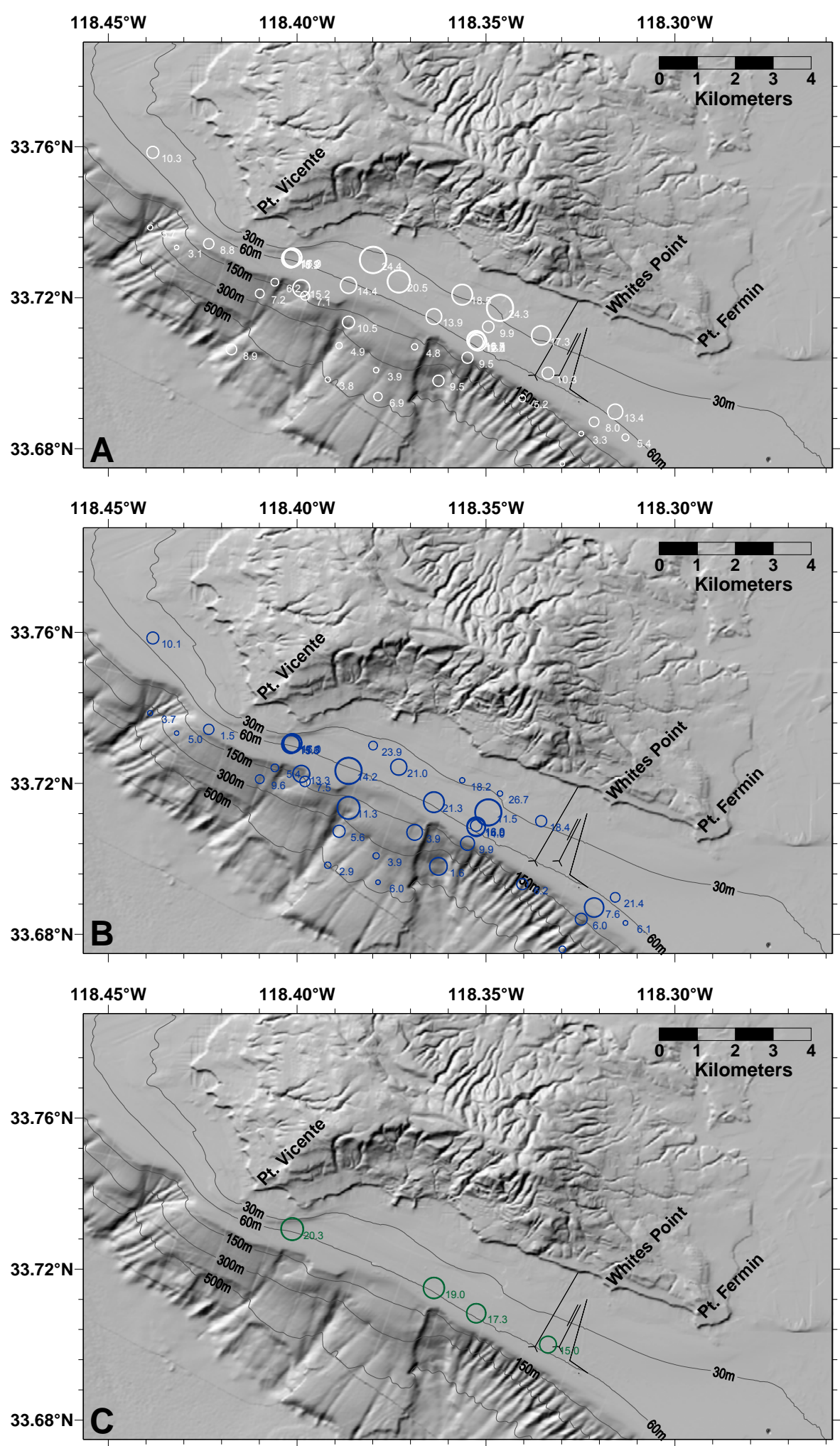

Figure 19. Shaded relief maps showing percent $p, p^{\prime}$-DDMU in box cores collected by the USGS in 1992: $(A)$ 0-4 cm, $(B) 0-8 \mathrm{~cm},(C)$ whole-core. Note: symbols are scaled (linearly) to numerical value of percent $p, p^{\prime}$-DDMU. 

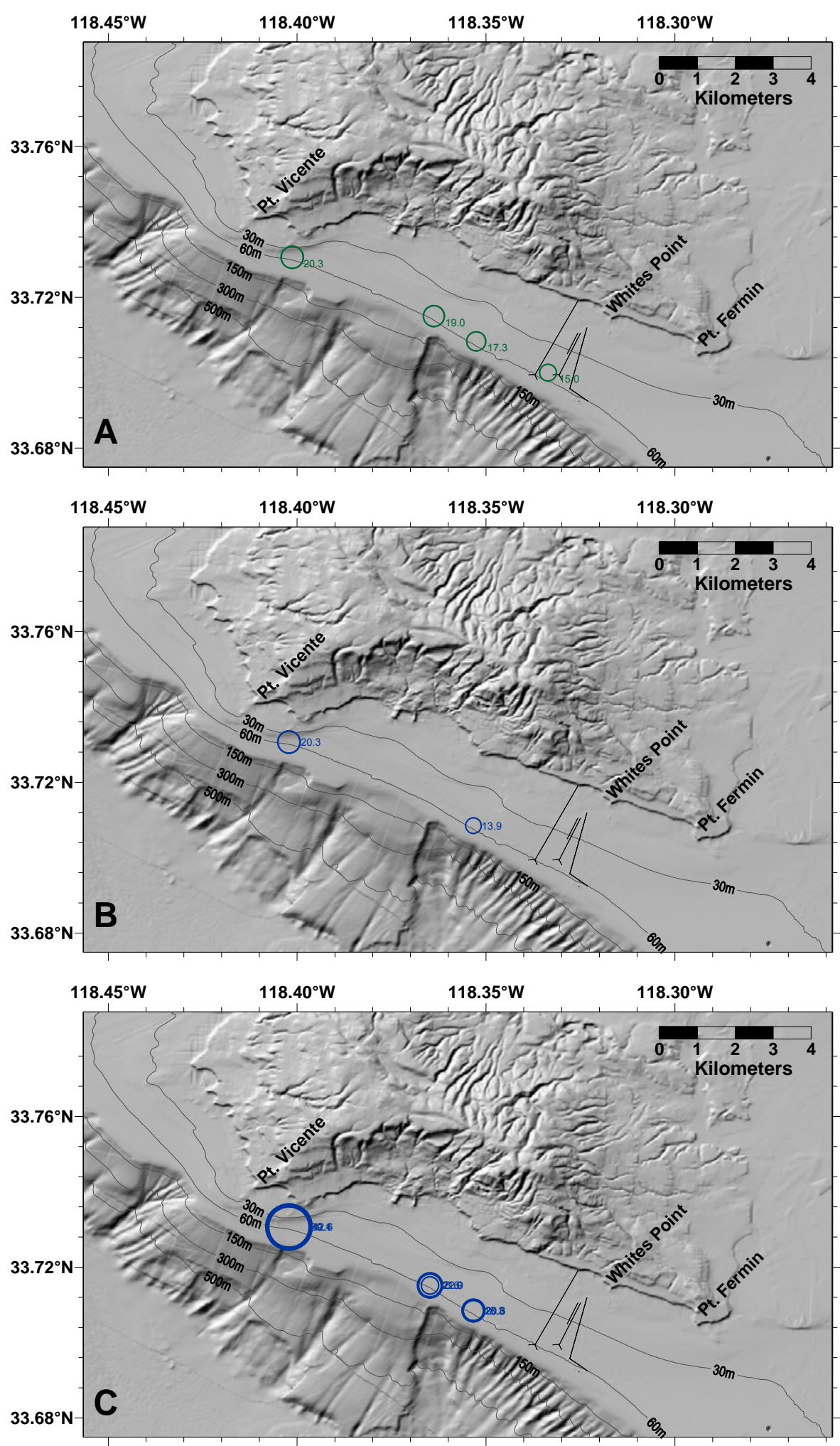

Figure 20. Shaded relief maps showing percent $p, p^{\prime}$-DDMU in cores collected by the: $(A)$ USGS-1992, (B) LACSD-1991, (C) LACSD-2005. LACSD = Los Angeles County Sanitation Districts. Note: symbols are scaled (linearly) to numerical value of percent $p, p^{\prime}$-DDMU 

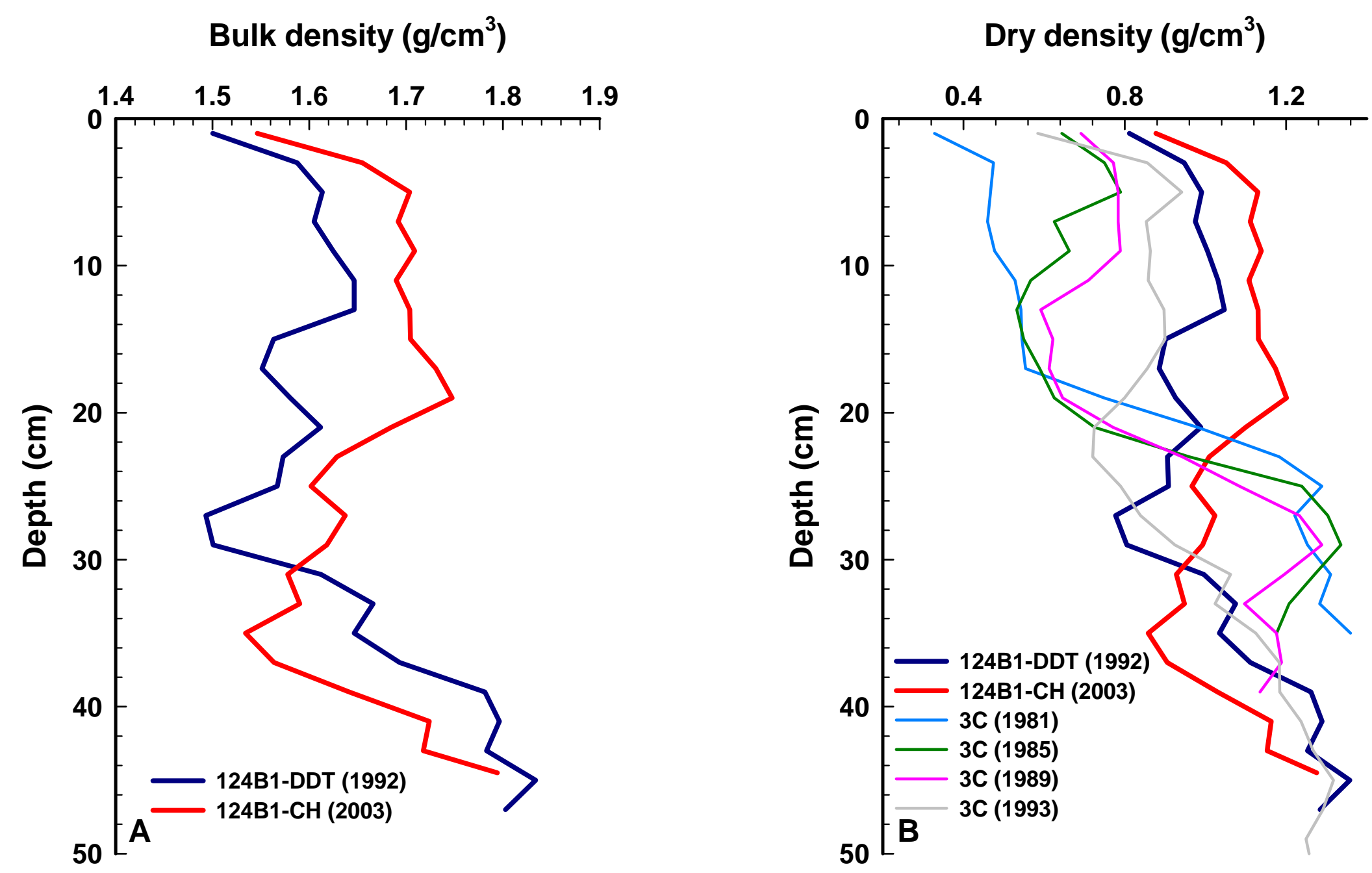

Figure 21. Graphs showing vertical density profiles for 124B1 cores collected by the USGS in 1992 and 2003 near station 3C: $(A)$ bulk density, $(B)$ dry density (includes data from the LACSD at station 3C). LACSD = Los Angeles County Sanitation Districts. 
*C28/hopane ratio

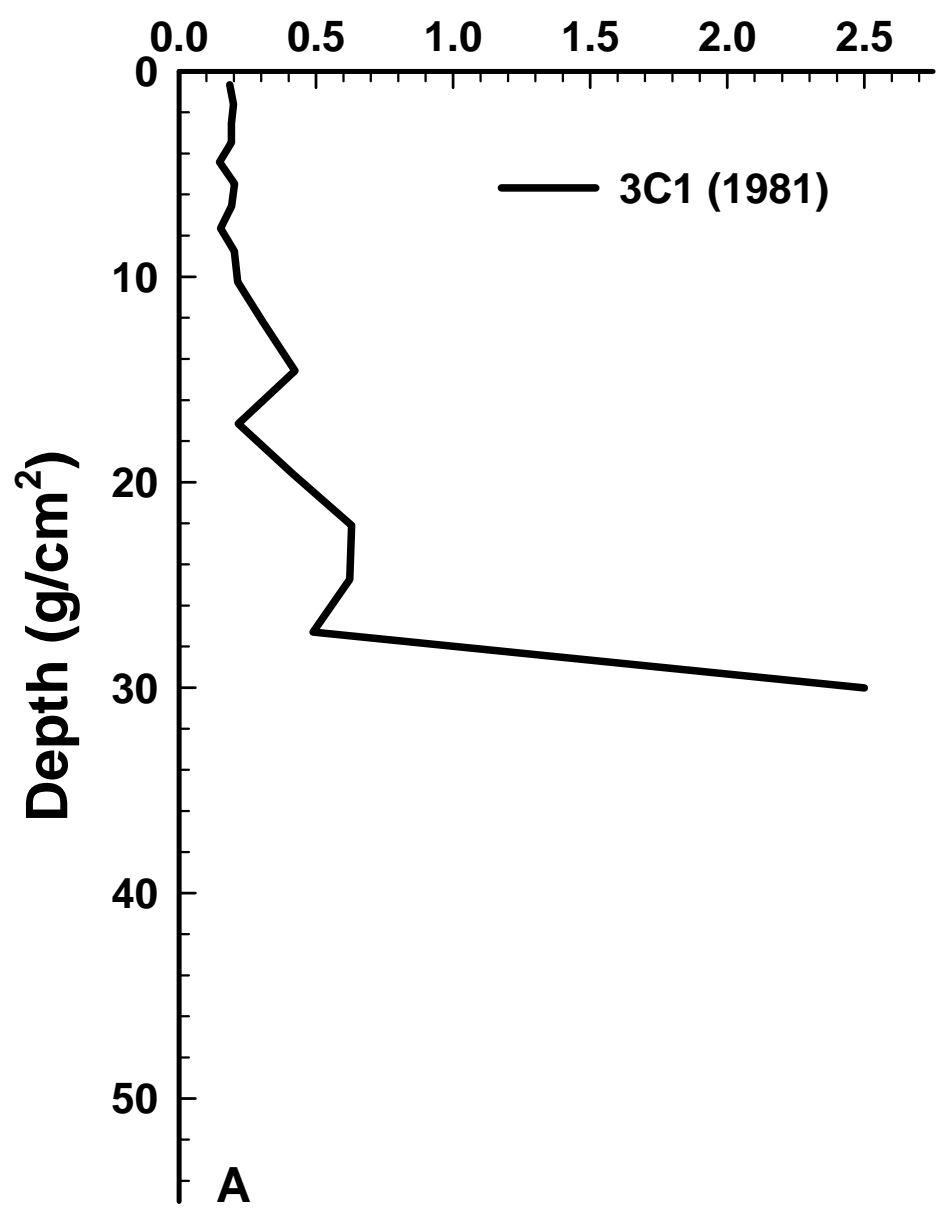

*C28/hopane ratio

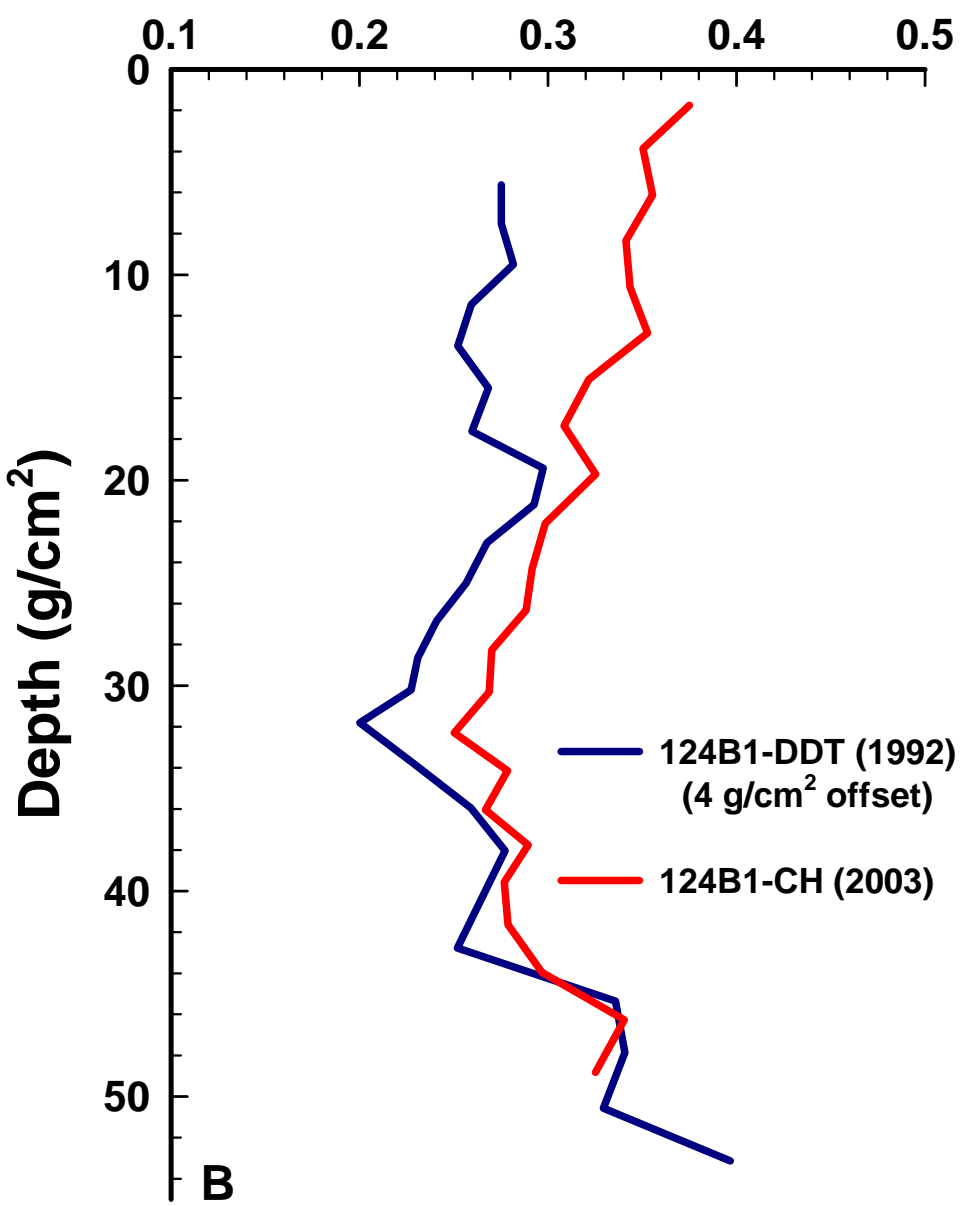

Figure 22. Graphs showing vertical profiles of the ${ }^{*} \mathrm{C} 28$ /hopane ratio in $(A)$ the $19813 \mathrm{C} 1$ core, and $(B)$ the 124B1-DDT (1992) and 124B1$\mathrm{CH}(2003)$ cores. See table 2 for station locations for these cores. ${ }^{*} \mathrm{C} 28=17 \alpha(\mathrm{H}), 21 \beta(\mathrm{H})-28,30$-bisnorhopane. Core profiles in panel $B$ are aligned $\left(4-\mathrm{g} / \mathrm{cm}^{2}\right.$ offset). 

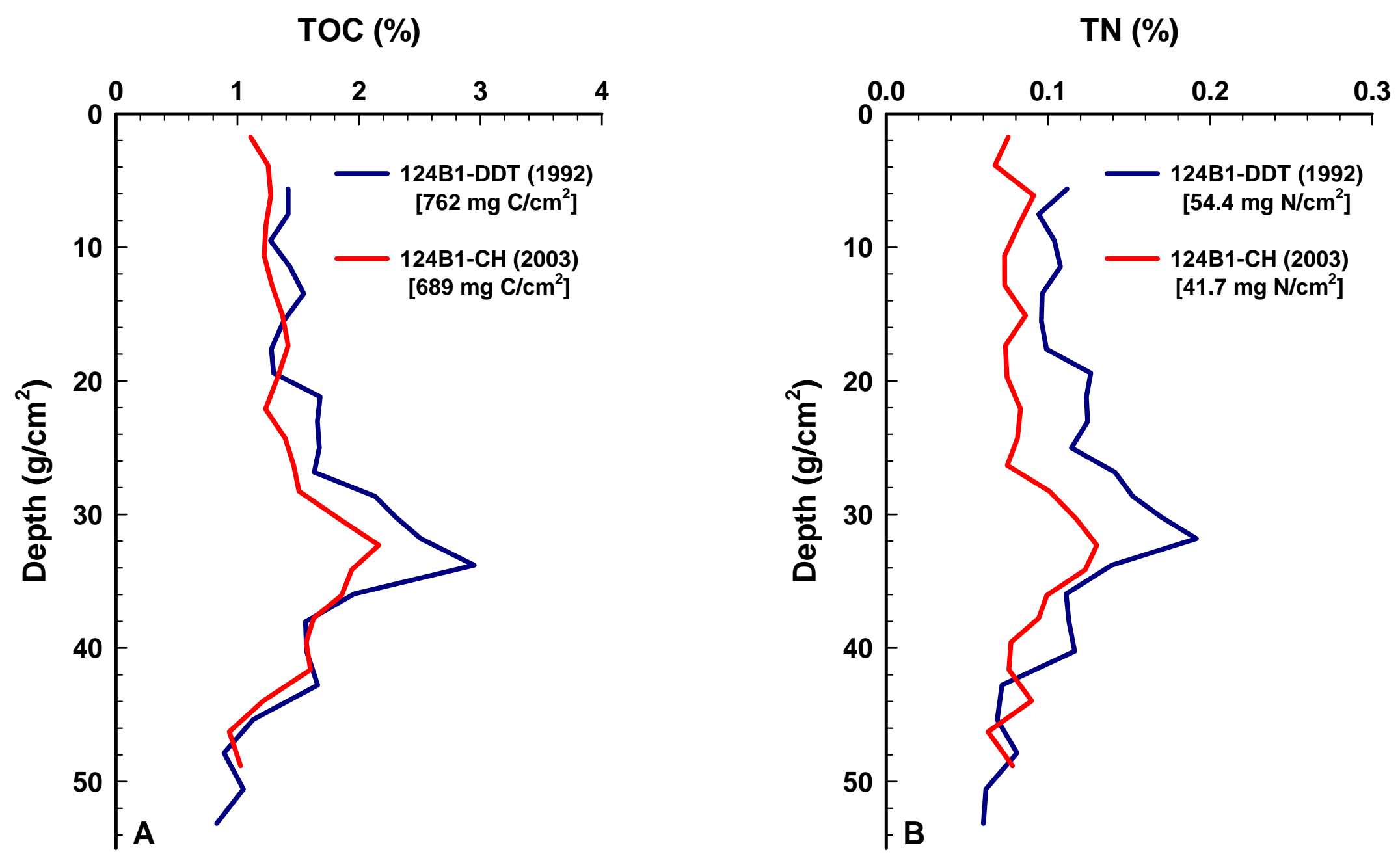

Figure 23. Graphs showing vertical concentration profiles of $(A)$ total organic carbon (TOC), and $(B)$ total nitrogen (TN) in sediment cores collected by the USGS near station 3C in 1992 and 2003. Whole-core inventories are given in brackets. Estimated method detection limits cannot be seen because of $\mathrm{x}$-axis scaling. Core profiles are aligned $\left(4-\mathrm{g} / \mathrm{cm}^{2}\right.$ offset). 


\section{TEO $(\mathrm{mg} / \mathrm{g})$}

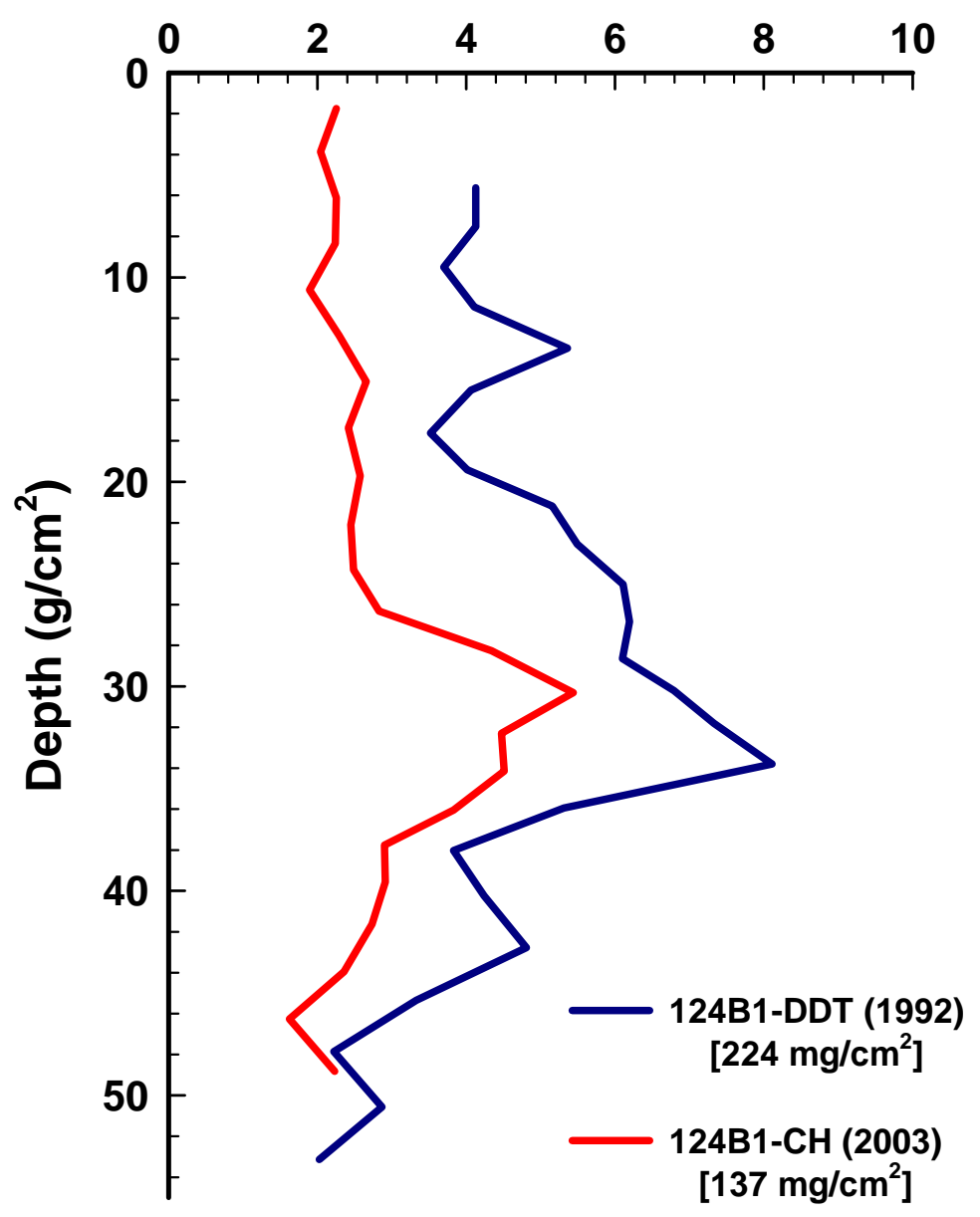

Figure 24. Graphs showing vertical concentration profiles of total extractable organics (TEO) in sediment cores collected by the USGS near station 3C in 1992 and 2003. Whole-core inventories are given in brackets. Core profiles are aligned $\left(4-\mathrm{g} / \mathrm{cm}^{2}\right.$ offset). 


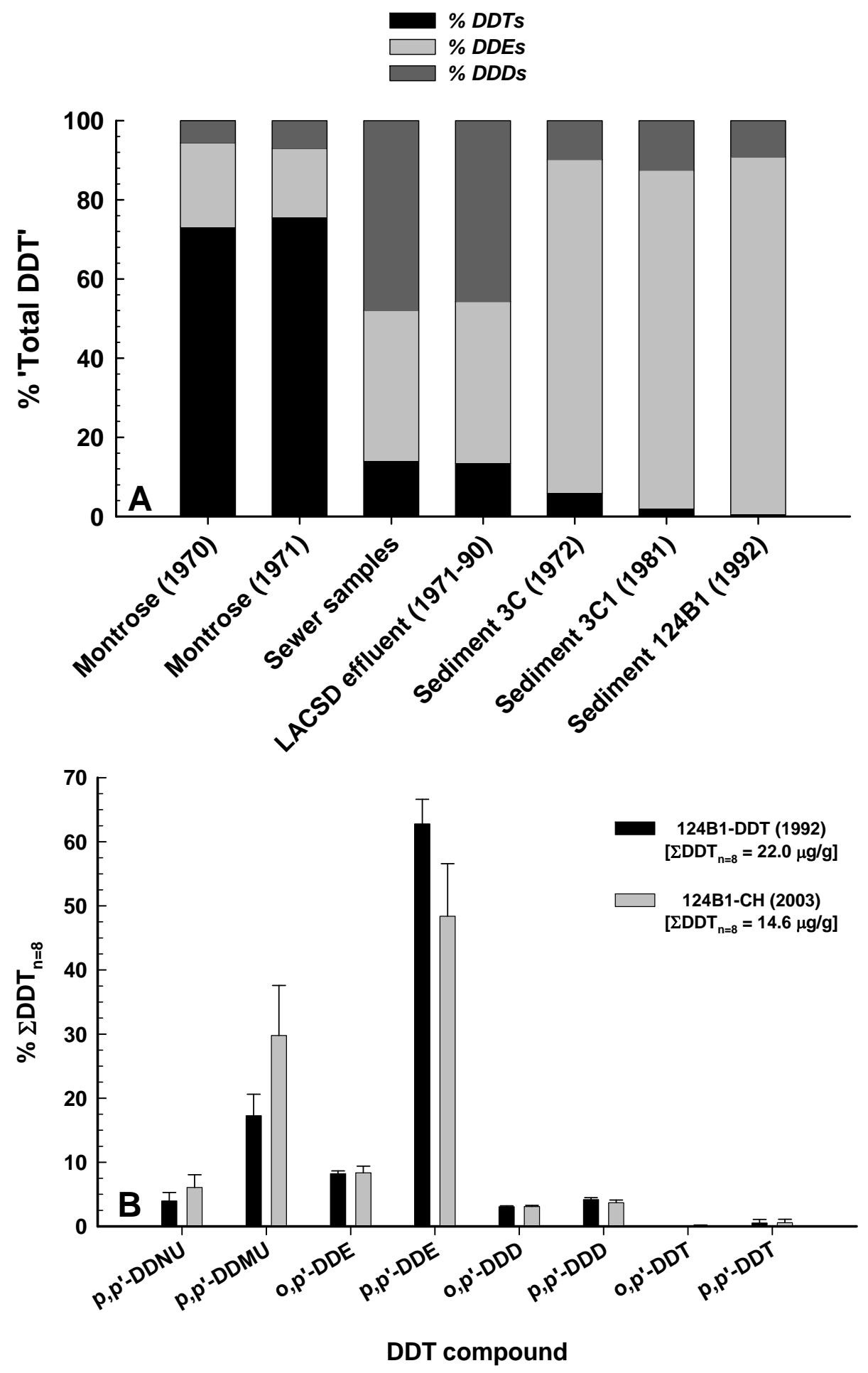

Figure 25. Graphs showing average composition of DDTs in (A) Montrose effluent, sewer samples, LACSD effluent, and sediments from the Palos Verdes Shelf (after Eganhouse and others, 2000), and (B) cores collected by the USGS near station 3C in 1992 and 2003. Maximum concentrations $\left(\sum D D T_{n=8}\right)$ in 124B1 cores are given in brackets. Error bars are one standard deviation. LACSD $=$ Los Angeles County Sanitation Districts. 


\section{B1-DDT (1992)}

DDX concentration $(\mu \mathrm{g} / \mathrm{g})$

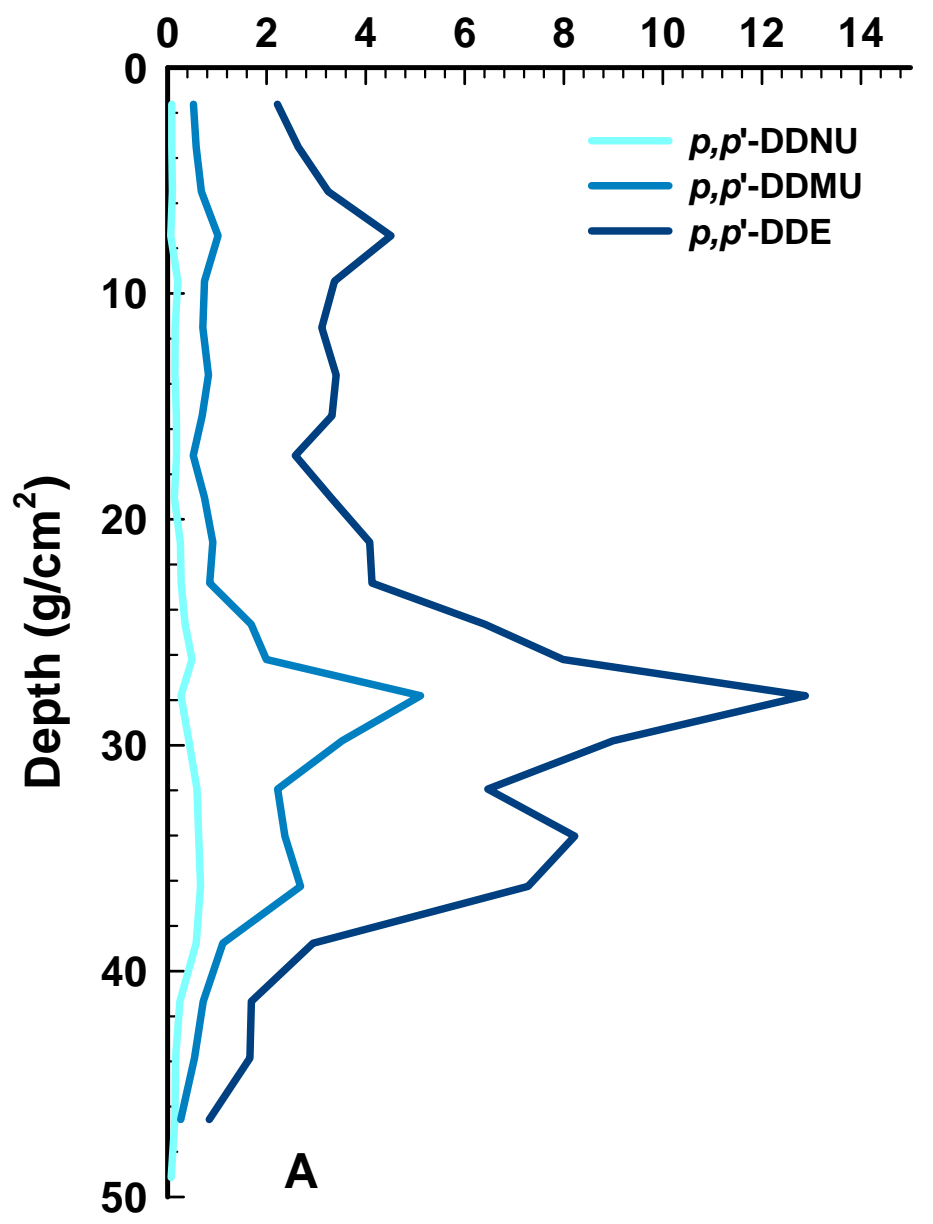

124B1-CH (2003)

DDX concentration $(\mu \mathrm{g} / \mathrm{g})$

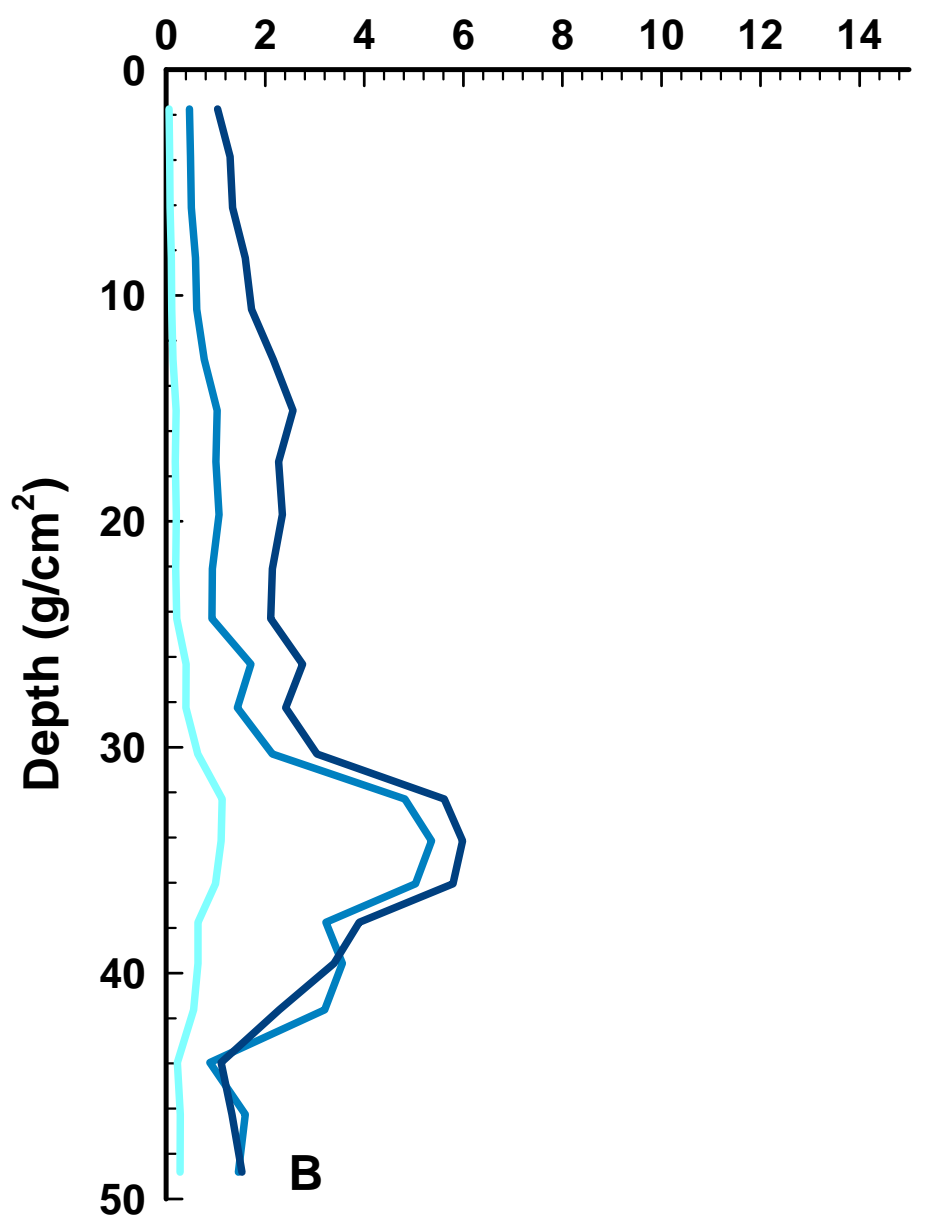

Figure 26. Graphs showing vertical concentration profiles of three DDT compounds in cores collected by the USGS near station $3 C$ in (A) 1992, and (B) 2003. Estimated method detection limits cannot be seen because of $x$-axis scaling. 

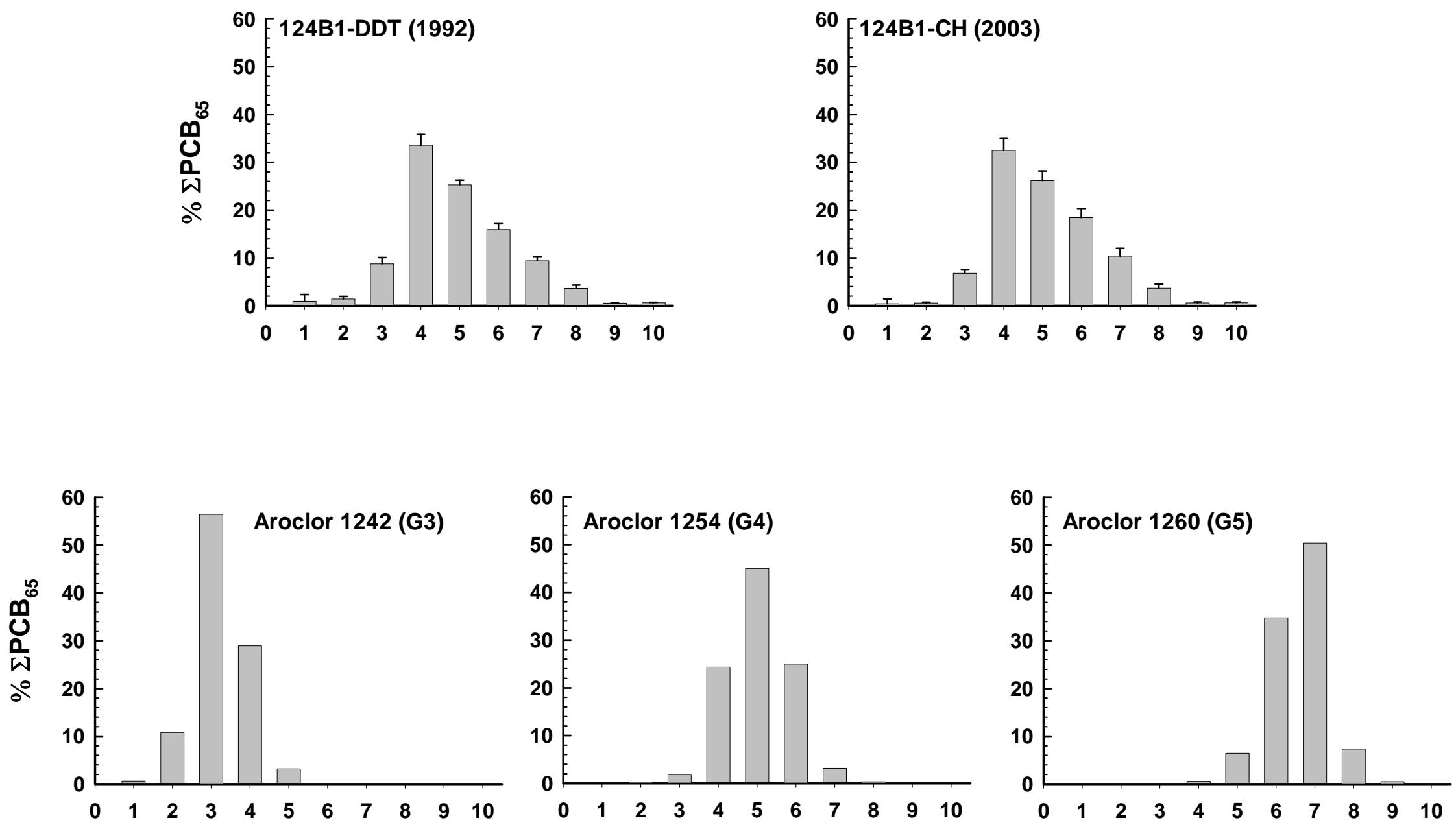

Number of chlorine atoms

Figure 27. Graphs showing distribution of PCBs by chlorination level in cores 124B1-DDT (1992) and 124B1-CH (2003) along with distributions found in three common Aroclors (Frame and others 1996). Data for cores and Aroclors include sixty-eight congeners free of major interference in the 124B1 cores (see table 3). Aroclor sources are indicated in parentheses as given in Frame and others (1996). Error bars for sediment core data are 1 standard deviation. 

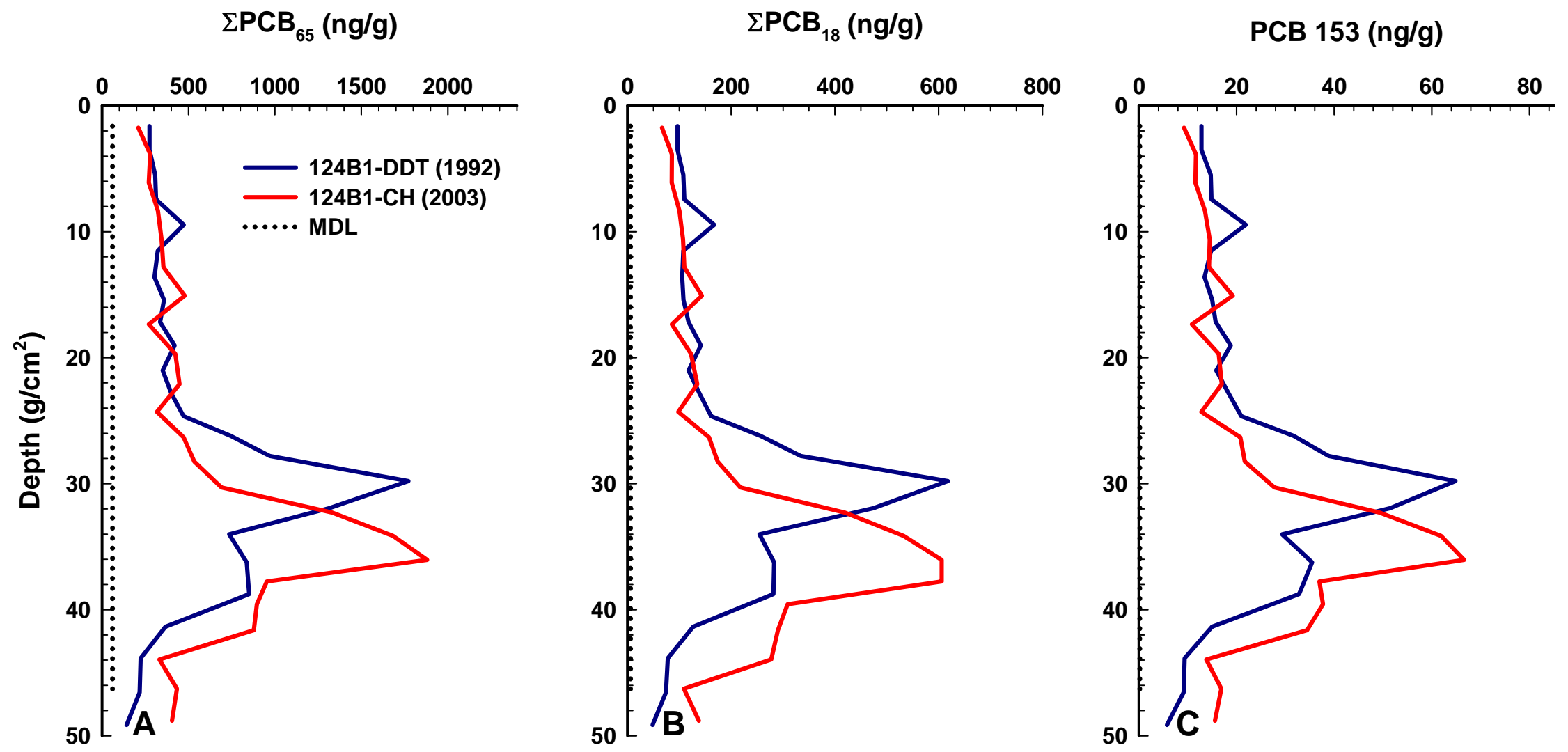

Figure 28. Graphs showing vertical concentration profiles of PCB congeners in cores collected by the USGS near station 3C in 1992 and 2003: $(A)$ sum of 65 PCB peaks (68 congeners) free of major interference $\left(\Sigma P C B_{65}\right),(B)$ sum of 18 interference-free PCB congeners $\left(\Sigma \mathrm{PCB}_{18}\right)$, and $(C) \mathrm{PCB}$ congener 153 (see table 3). Estimated method detection limits (MDLs) are indicated by dotted lines. Note: $\mathrm{X}-$ axes are scaled differently. Core profiles are not aligned. PCBs = polychlorinated biphenyls. 

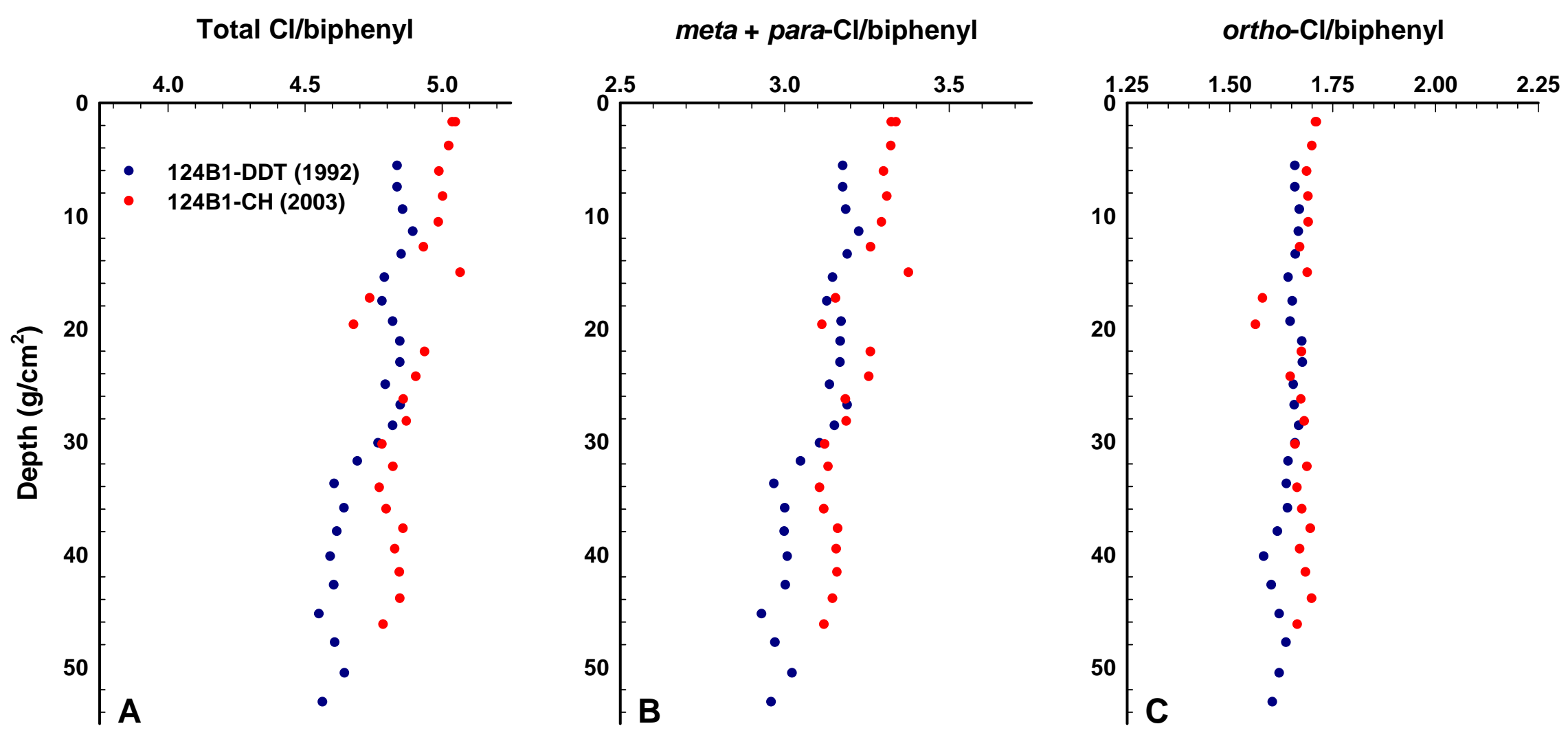

Figure 29. Graphs showing vertical profiles of molar abundance of chlorine/biphenyl in the 124B1-DDT (1992) and 124B1-CH (2003) cores: $(A)$ total chlorines/biphenyl, $(B)$ meta- + para-chlorines/biphenyl, and $(C)$ ortho-chlorines/biphenyl. Core profiles are aligned (4$\mathrm{g} / \mathrm{cm}^{2}$ offset). 

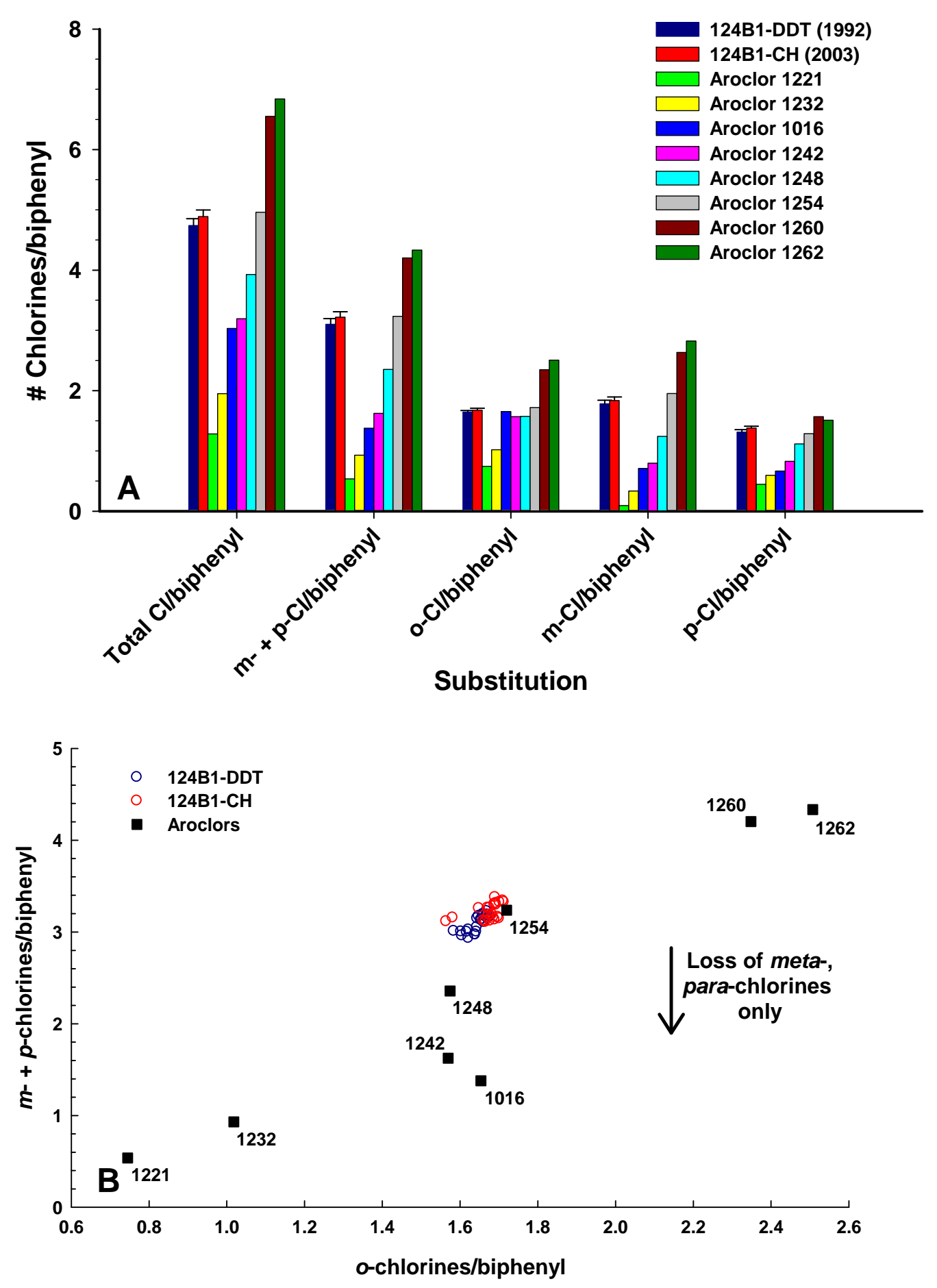

Figure 30. Graphs showing $(A)$ total, meta- + para-, ortho-, meta- and para-chlorine abundances, and $(B)$ meta- + para-chlorine abundance versus ortho-chlorine abundances in 124B1 cores (average) and Aroclors based on 68 PCB congeners (see table 3). Aroclor data are from Frame and others (1996). PCB = polychlorinated biphenyl. 


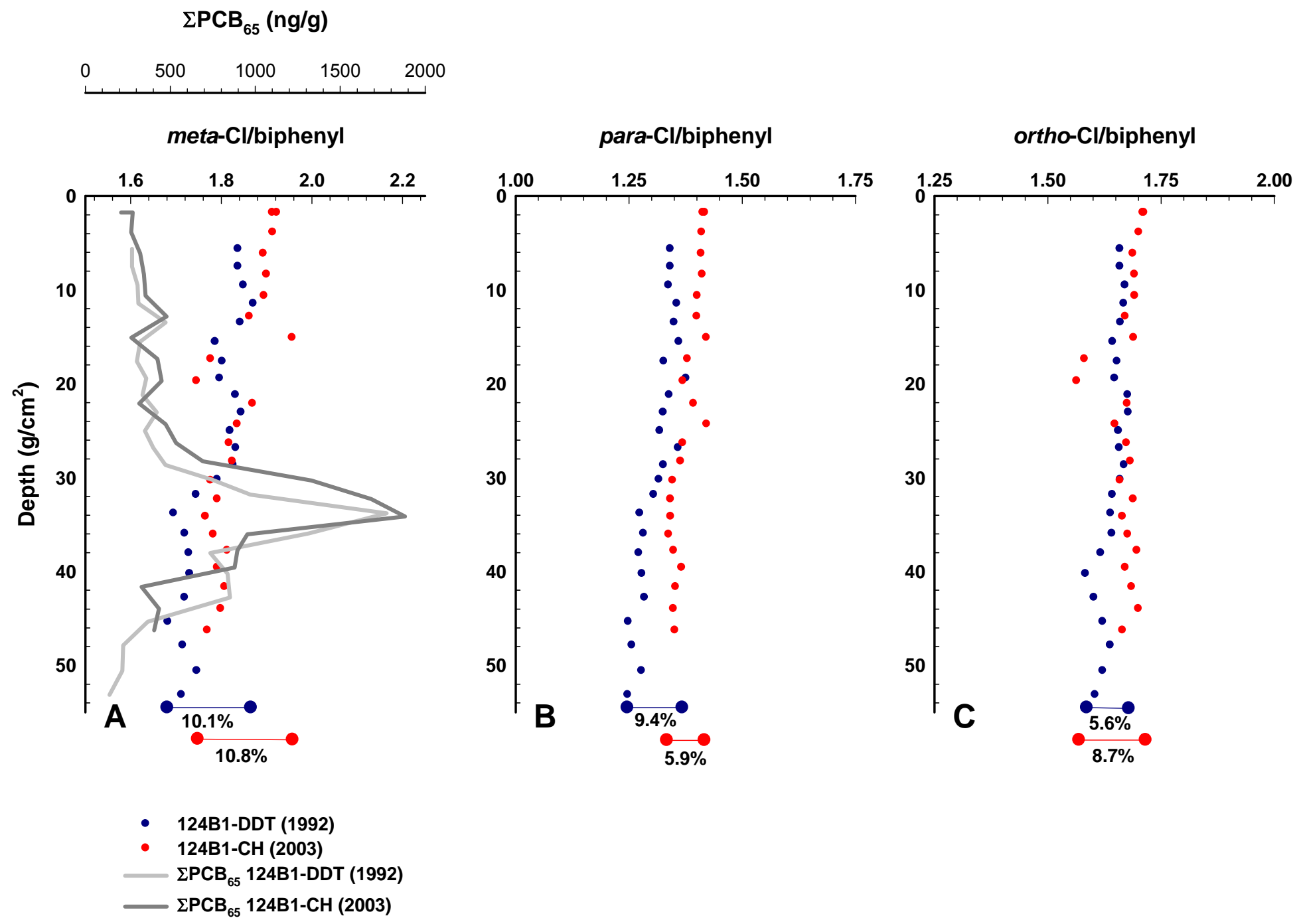

Figure 31. Graphs showing vertical profiles of $(A)$ meta- $\mathrm{Cl} /$ biphenyl abundance and $\Sigma \mathrm{PCB}_{65}$ concentration, $(B)$ para-Cl/biphenyl abundance, and $(C)$ ortho-Cl/biphenyl abundance in cores 124B1-DDT (1992) and 124B1-CH (2003). Within-core ranges of Cl/biphenyl abundances are given below profiles. Core profiles are aligned $\left(4-\mathrm{g} / \mathrm{cm}^{2}\right.$ offset). $\mathrm{PCB}=$ polychlorinated biphenyl 

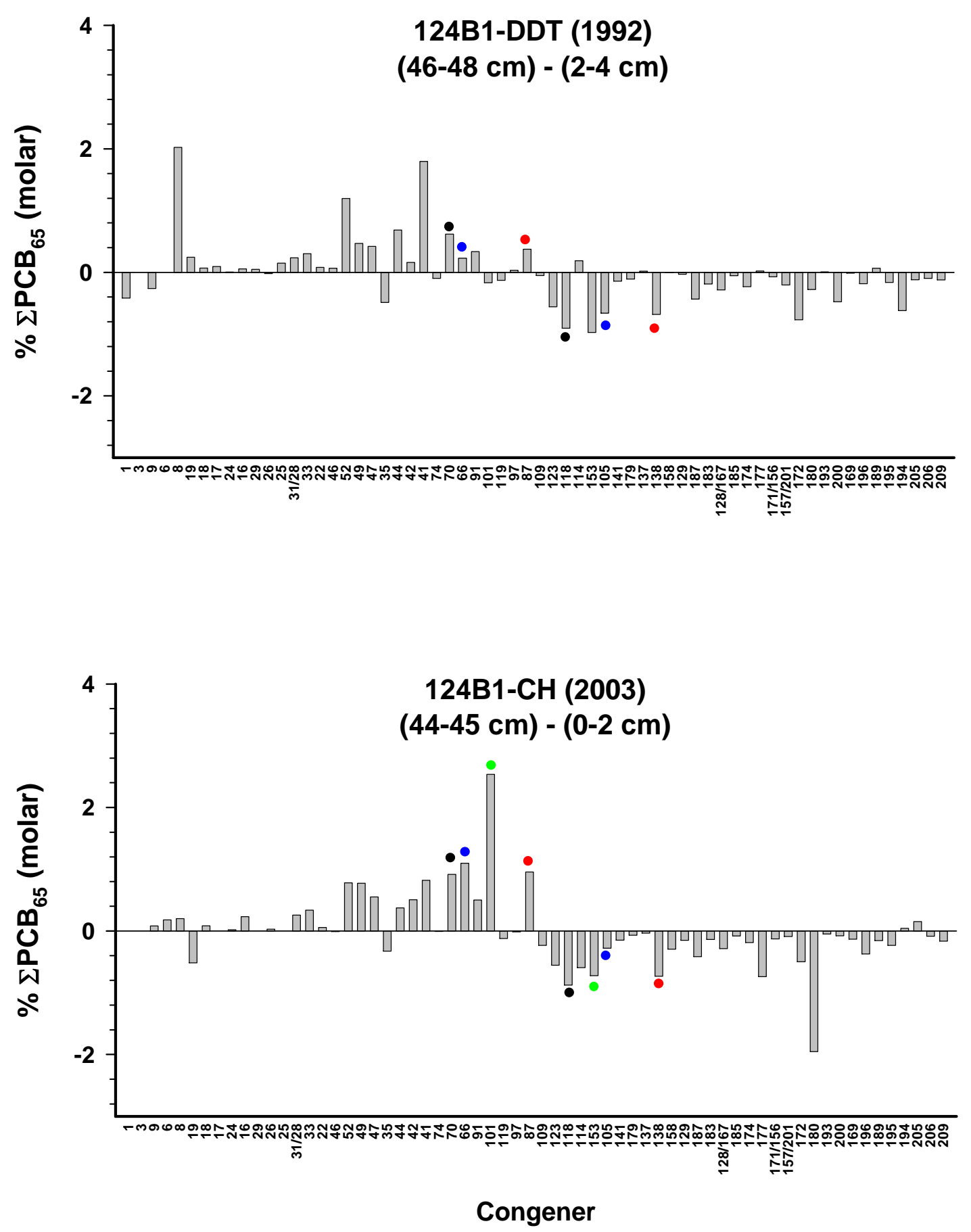

Figure 32. Graphs showing difference between molar composition of $\mathrm{PCB}$ congeners in sediments from cores 124B1-DDT (1992) and 124B1-CH (2003) at the bottom and tops of the cores. Data are shown for 65 congener peaks corresponding to $\Sigma \mathrm{PCB}_{65}$ (see table 3 ). Colored circles identify potential dechlorination parent-daughter pairs (see text for explanation). $\mathrm{PCB}=$ polychlorinated biphenyl. 

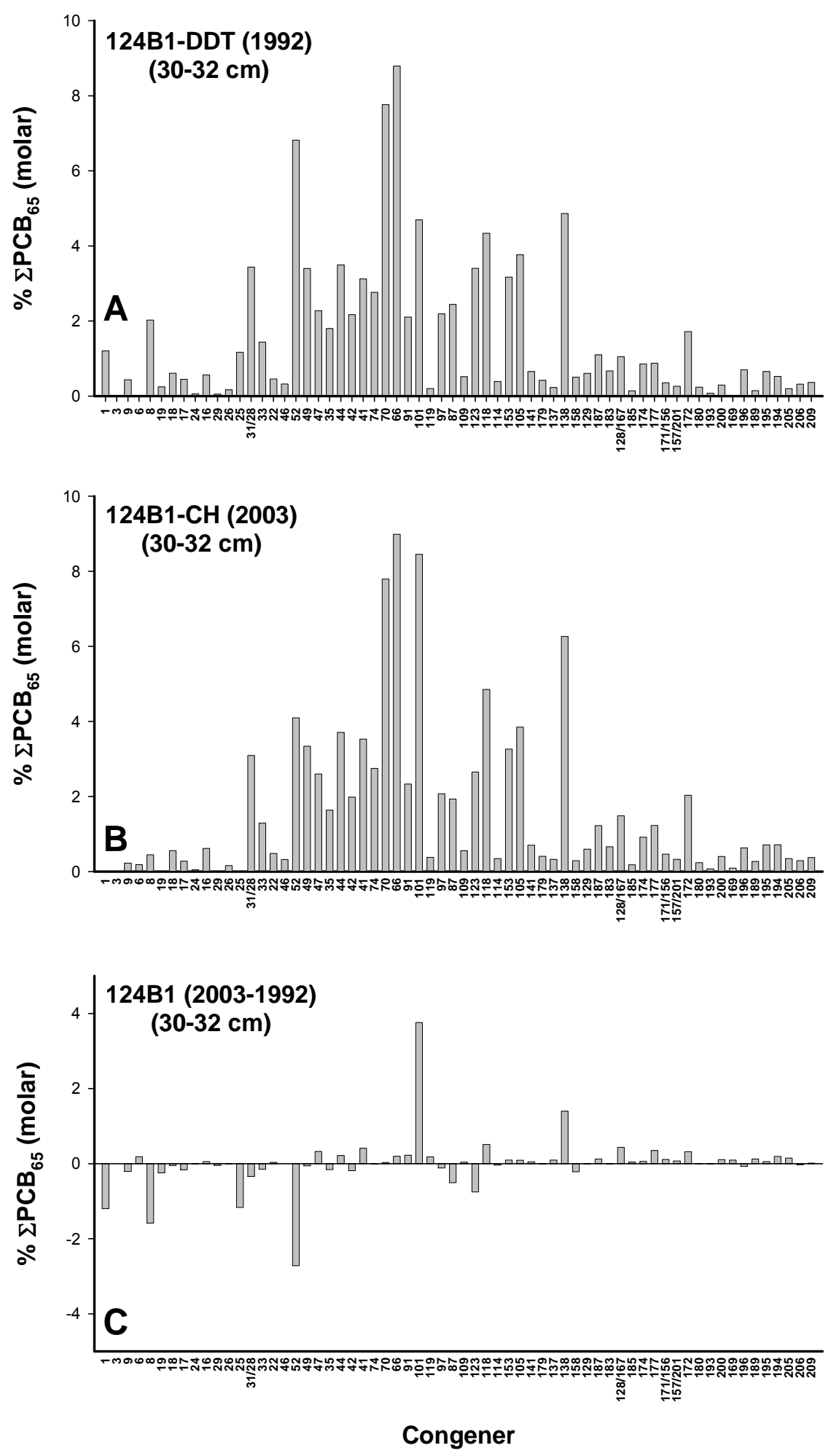

Figure 33. Graphs showing percent molar composition of PCBs in cores $(A)$ 124B1-DDT (1992), and $(B) 124 \mathrm{~B} 1-\mathrm{CH}(2003)$ at depths of maximum concentration $(30-32 \mathrm{~cm})$ along with $(C)$ plot of difference between the cores. Compositions are normalized to $\Sigma \mathrm{PCB}_{65} \cdot \mathrm{PCBs}=$ polychlorinated biphenyls. 


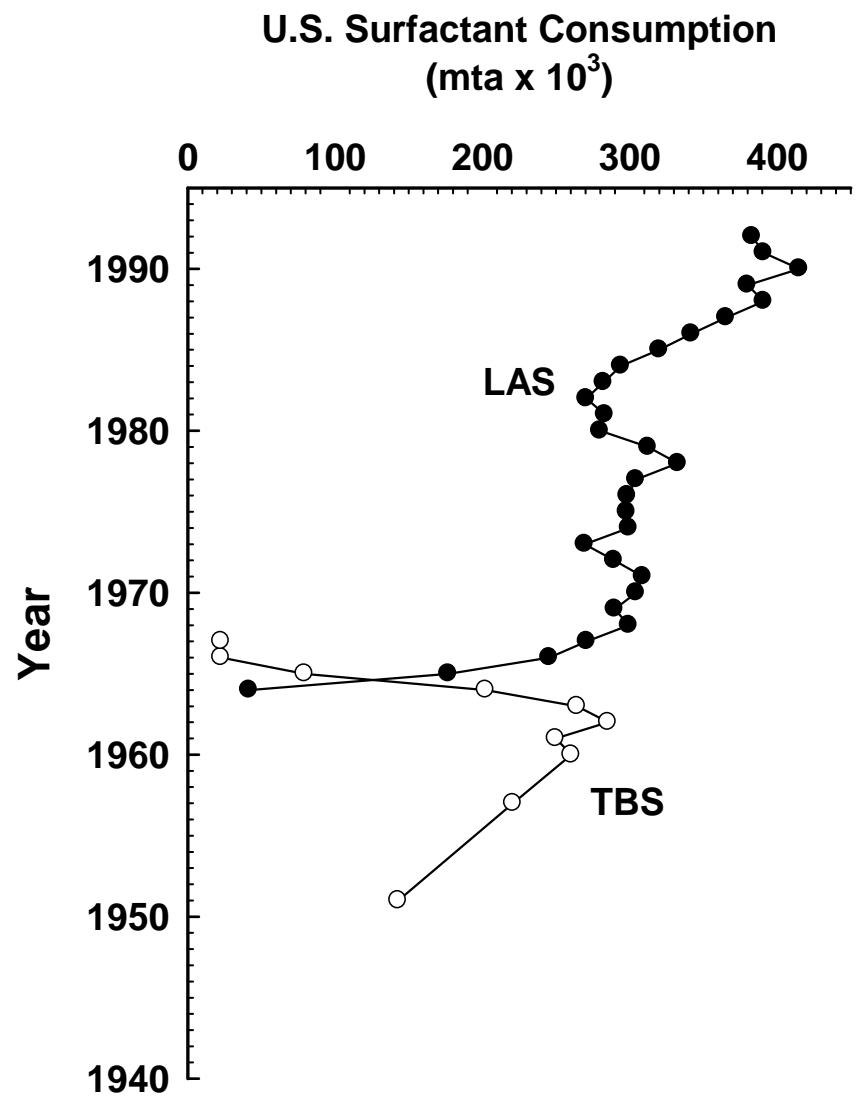

Figure 34. Graph showing synthetic alkylbenzene sulfonate surfactant consumption in the United States (1950-1992) (Modler 1994) $. \mathrm{mta}=$ metric tons per year. LAS $=$ linear alkylbenzene sulfonate. TBS = tetrapropylene benzene sulfonate. 

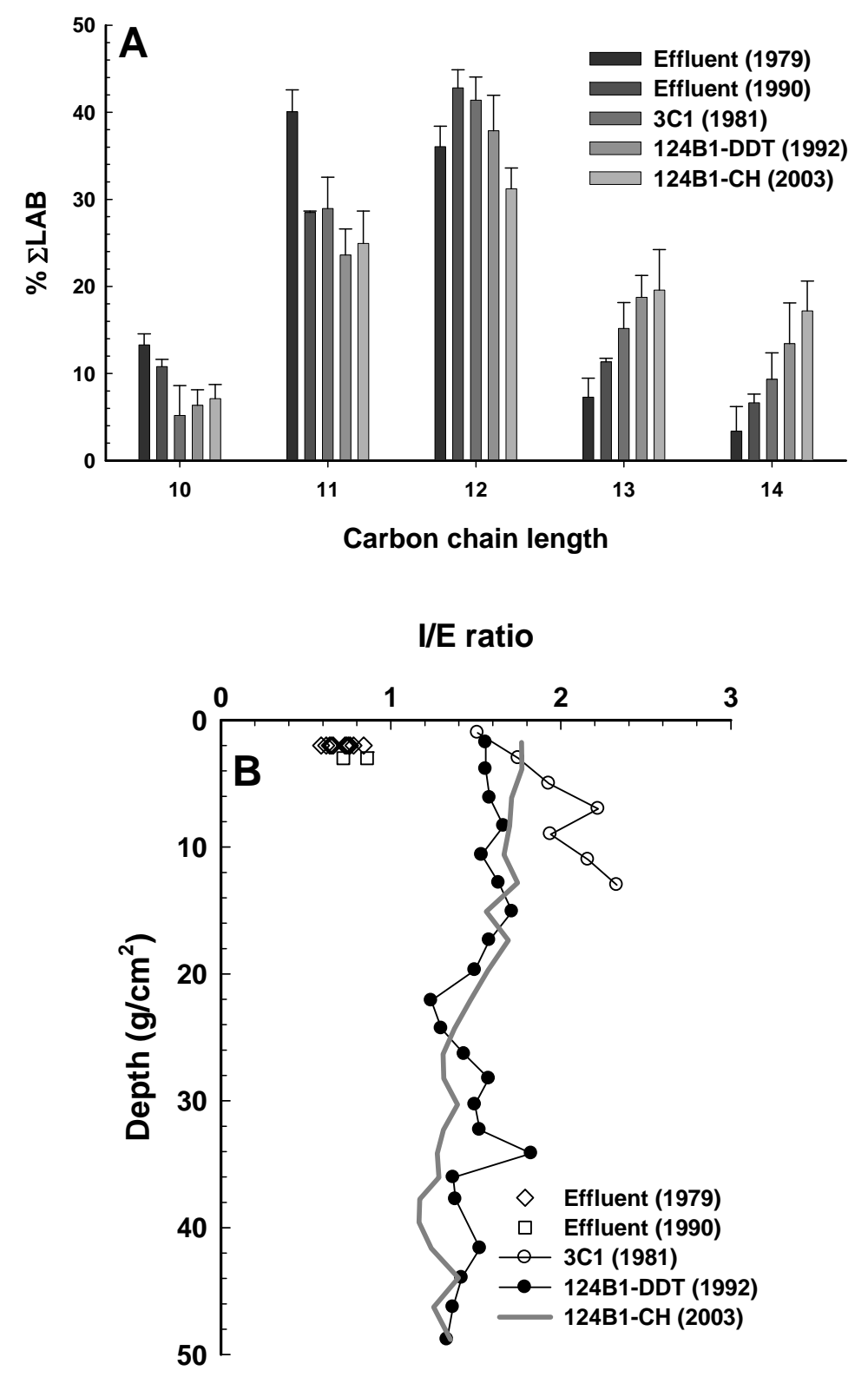

Figure 35. Graph showing $(A)$ alkyl chain length and $(B)$ dodecylbenzene isomer compositions of linear alkylbenzenes in sediment cores, LACSD effluent. Error bars are 1 standard deviation. Data for LACSD effluent: 1979 from Eganhouse and others (1983b), 1990 from Hendricks and Eganhouse (1992); 3C1 core: Eganhouse and others (1983b). Core profiles are not aligned. LACSD = Los Angeles County Sanitation Districts. I/E ratio $=\{[6-$ phenyldodecane $]+[5-$ phenyldodecane $]\} /\{[4-$ phenyldodecane] + [3-phenyldodecane] + [2-phenyldodecane]\} 

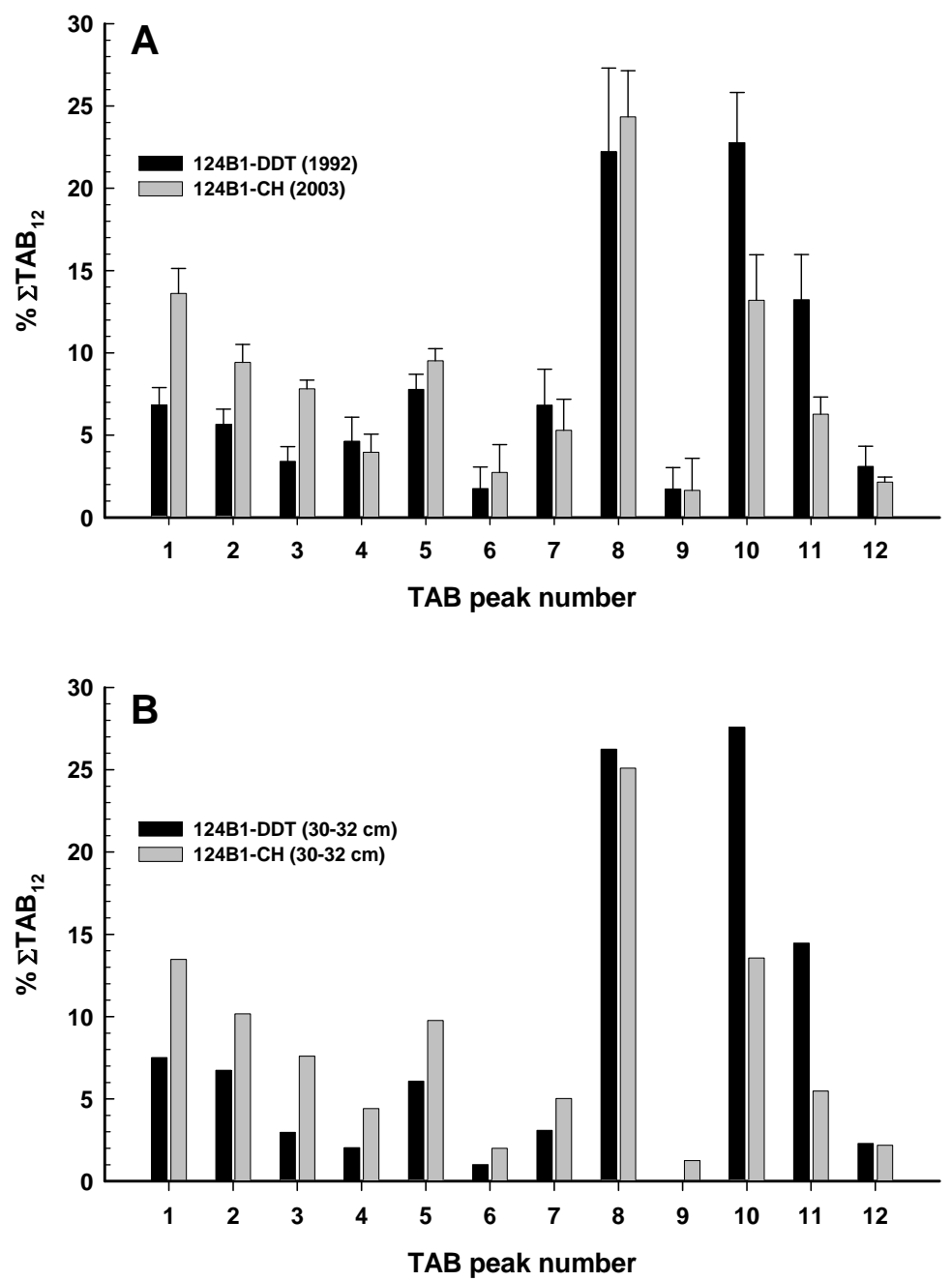

Figure 36. Graph showing composition of TABs in 124B1 cores: $(A)$ average for all depths (error bars $=1$ standard deviation), and $(B)$ abundance in section of maximum PCB/DDT concentration (30-32 $\mathrm{cm})$. TABs $=$ tetrapropylene-based alkylbenzenes, $\mathrm{PCB}=$ polychlorinated biphenyl, DDT = dichlorodiphenyltrichloroethane. 

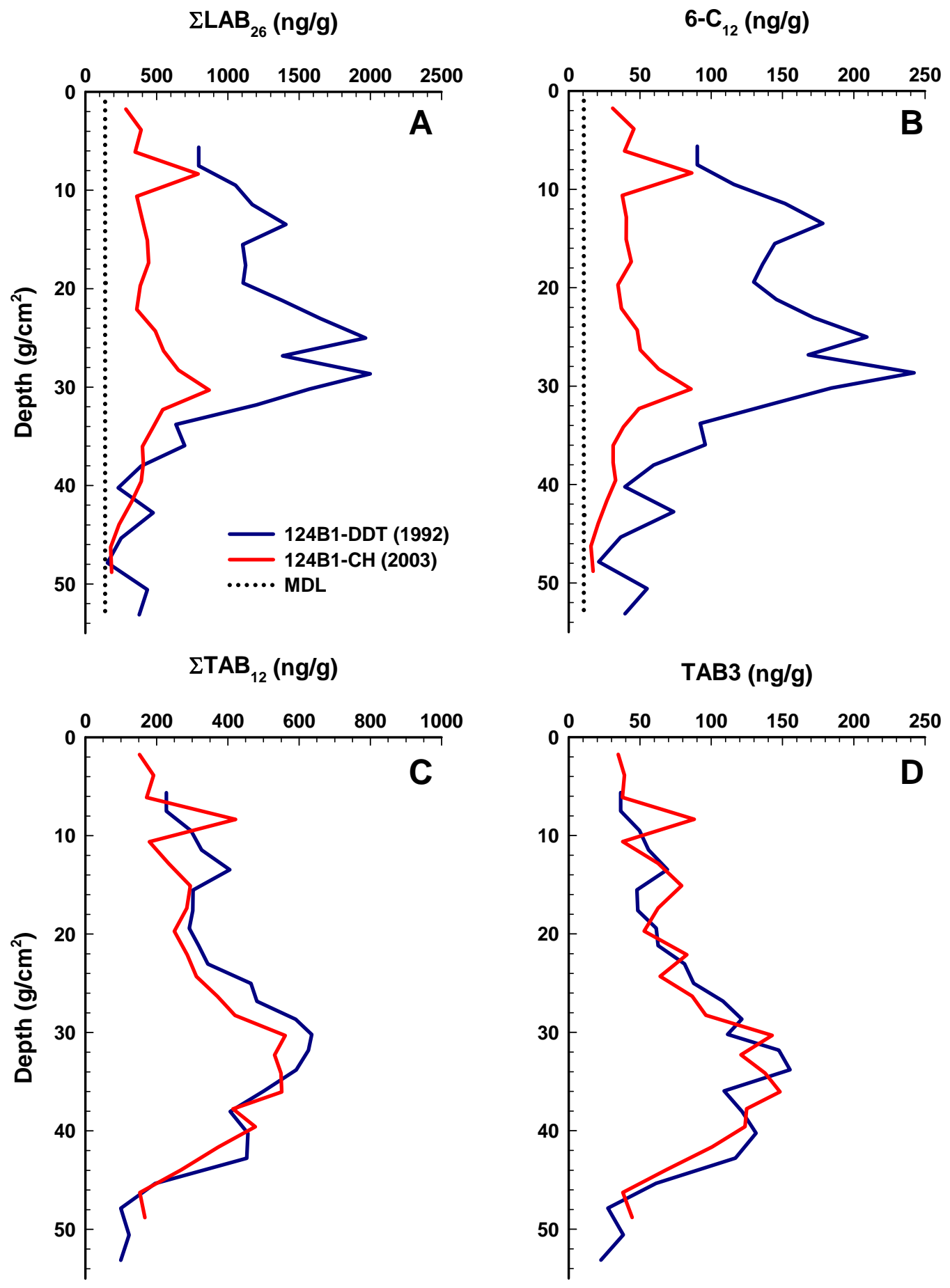

Figure 37. Graph showing vertical concentration profiles of long-chain alkylbenzenes in cores collected by the USGS near station $3 C$ in 1992 and 2003: $(A)$ total linear alkylbenzenes $\left(\Sigma L B_{26}\right),(B) 6$ 6phenyldodecane $\left(6-C_{12}\right),(C)$ summed TAB analytes $\left(\Sigma T A B_{12}\right)$, and $(D)$ a persistent TAB (TAB3). Estimated method detection limits (MDLs) are indicated by the dotted lines. Note: $X$-axes are scaled differently. Core profiles are aligned $\left(4-\mathrm{g} / \mathrm{cm}^{2}\right.$ offset $) . T A B=$ tetrapropylene-based alkylbenzene. 

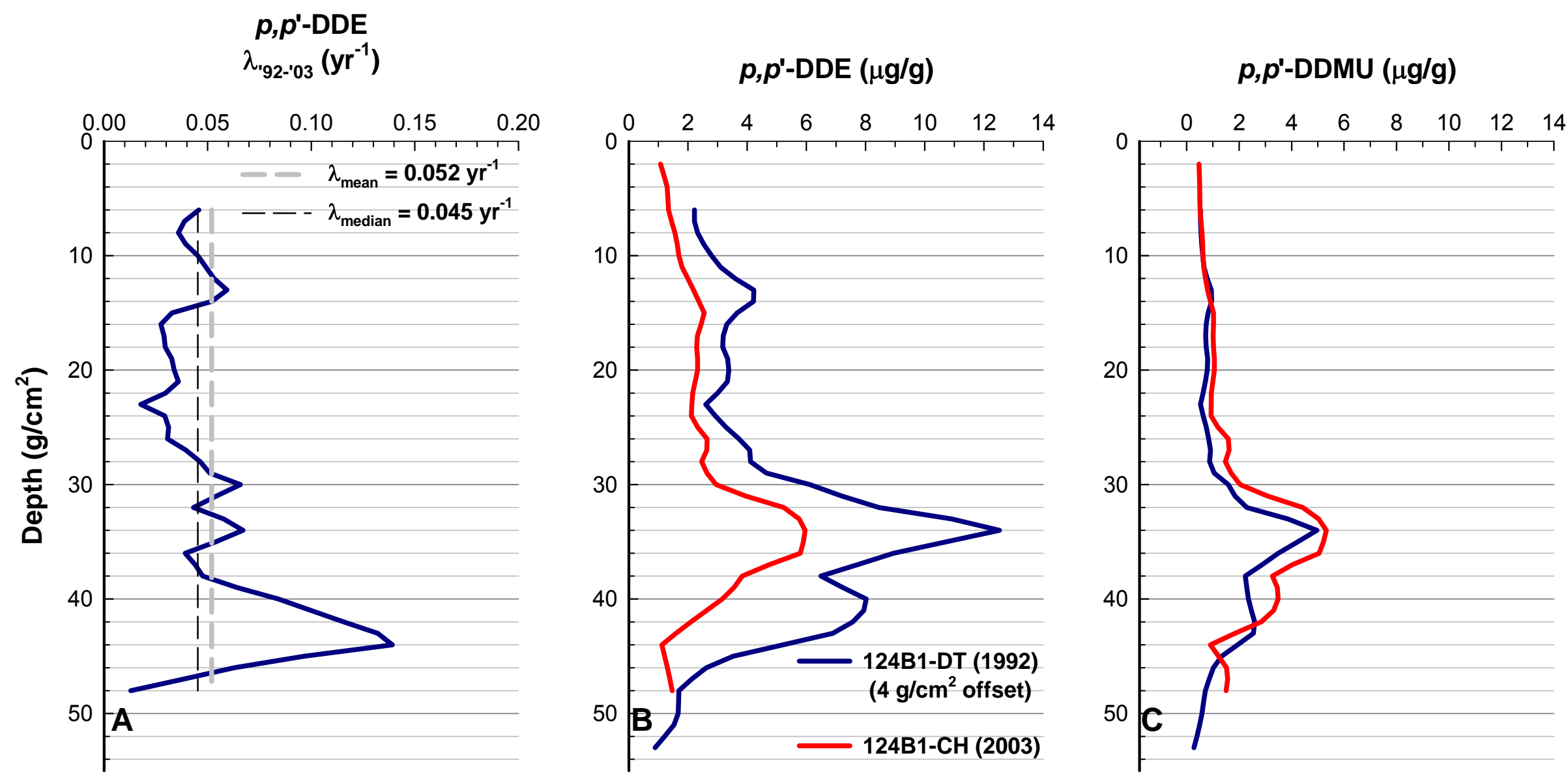

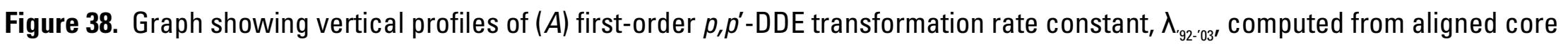
data, and $(B)$ concentration of $p, p^{\prime}$-DDE, and $(C) p, p^{\prime}$-DDMU in 1992, 2003 cores. Core profiles are aligned $\left(4-\mathrm{g} / \mathrm{cm}^{2}\right.$ offset). Uncertainty in estimated first-order $p, p^{\prime}$-DDE transformation rate constants is $0.019-0.025 \mathrm{yr}^{-1}$. 


\section{$p, p^{\prime}-$ DDE $(\mu g / g)$}

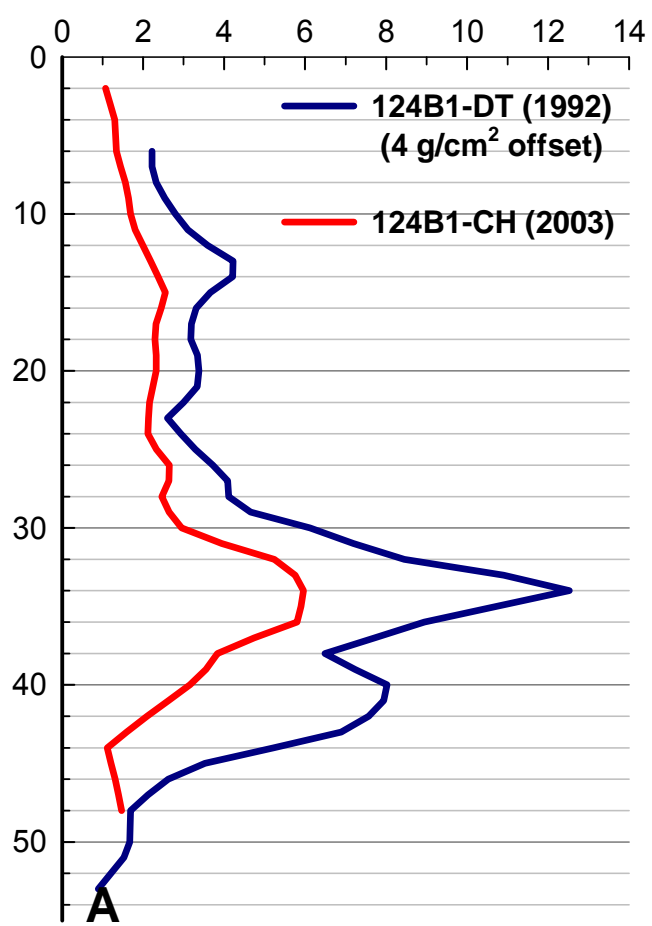

$p, p^{\prime}-$ DDMU $(\mu \mathrm{g} / \mathrm{g})$

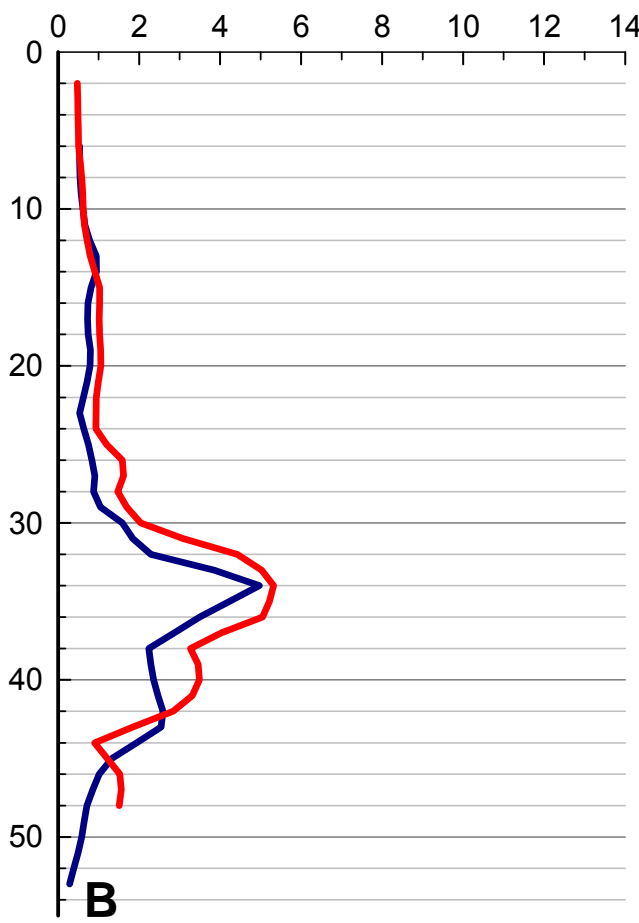

$p, p^{\prime}$-DDNU ( $\left.\mu \mathrm{g} / \mathrm{g}\right)$

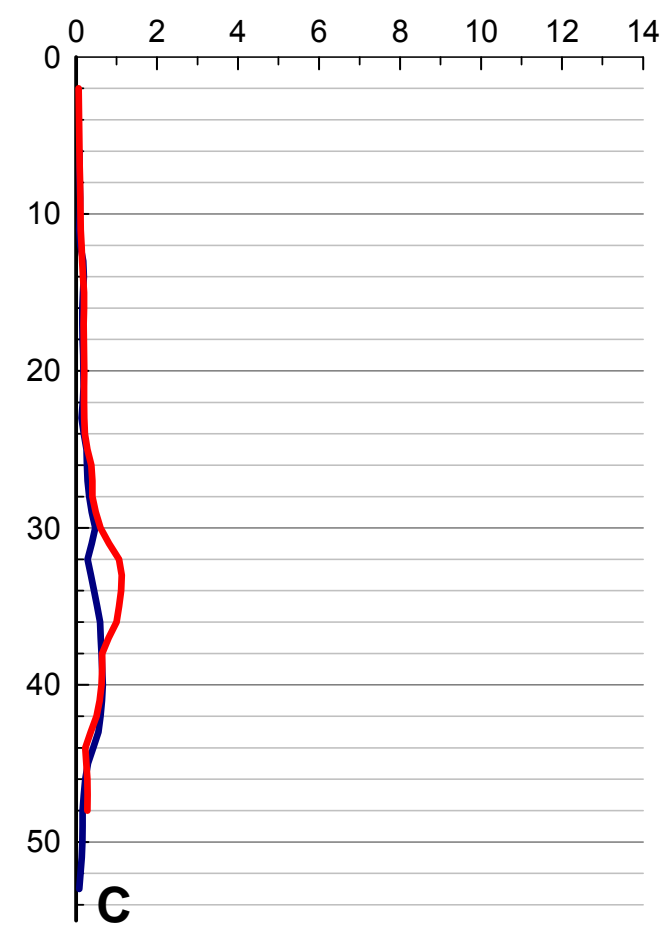

Figure 39. Graph showing vertical concentration profiles of $(A) p, p^{\prime}$-DDE, $(B) p, p^{\prime}$-DDMU, $(C) p, p^{\prime}$-DDNU in cores collected by the USGS near station $3 C$ in 1992 and 2003 . Core profiles are aligned $\left(4-\mathrm{g} / \mathrm{cm}^{2}\right.$ offset). 


\section{$\Sigma[\mathrm{DDE}, \mathrm{DDMU}, \mathrm{DDNU}]\left(\mu \mathrm{mol} / \mathrm{cm}^{2}\right)$}

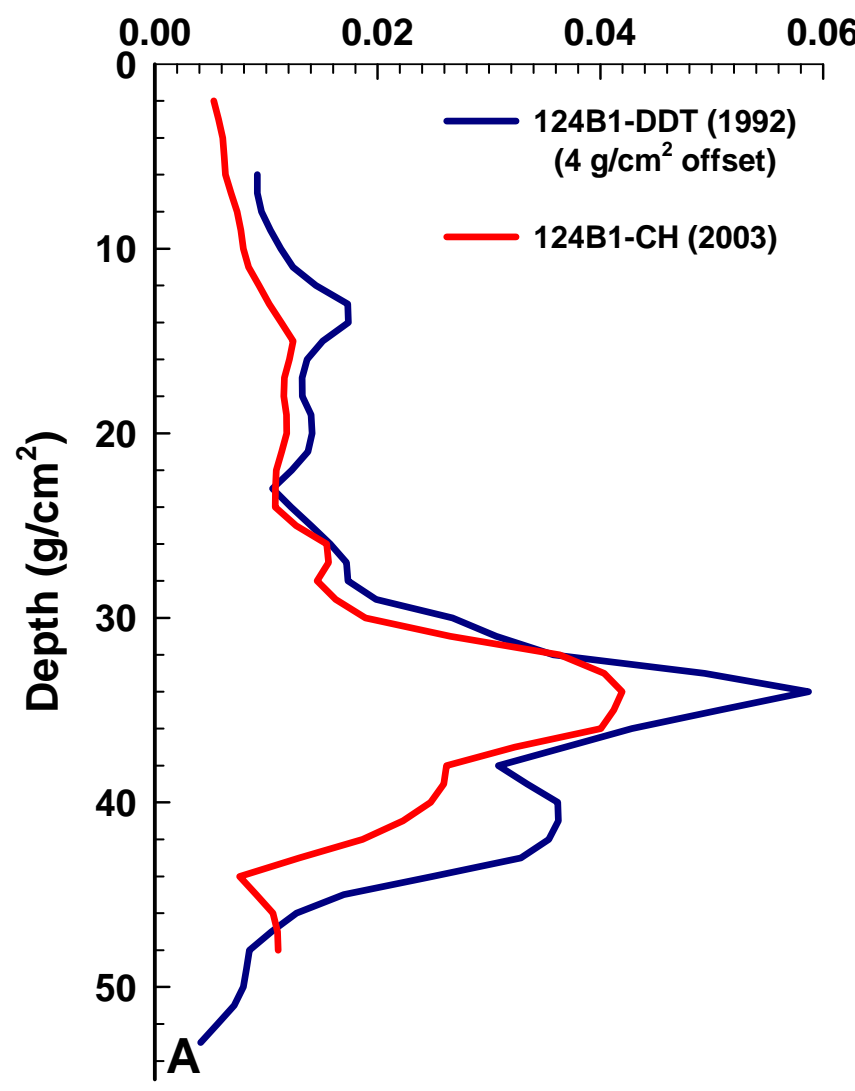

$\Delta_{\text {mass balance }}(\%)$

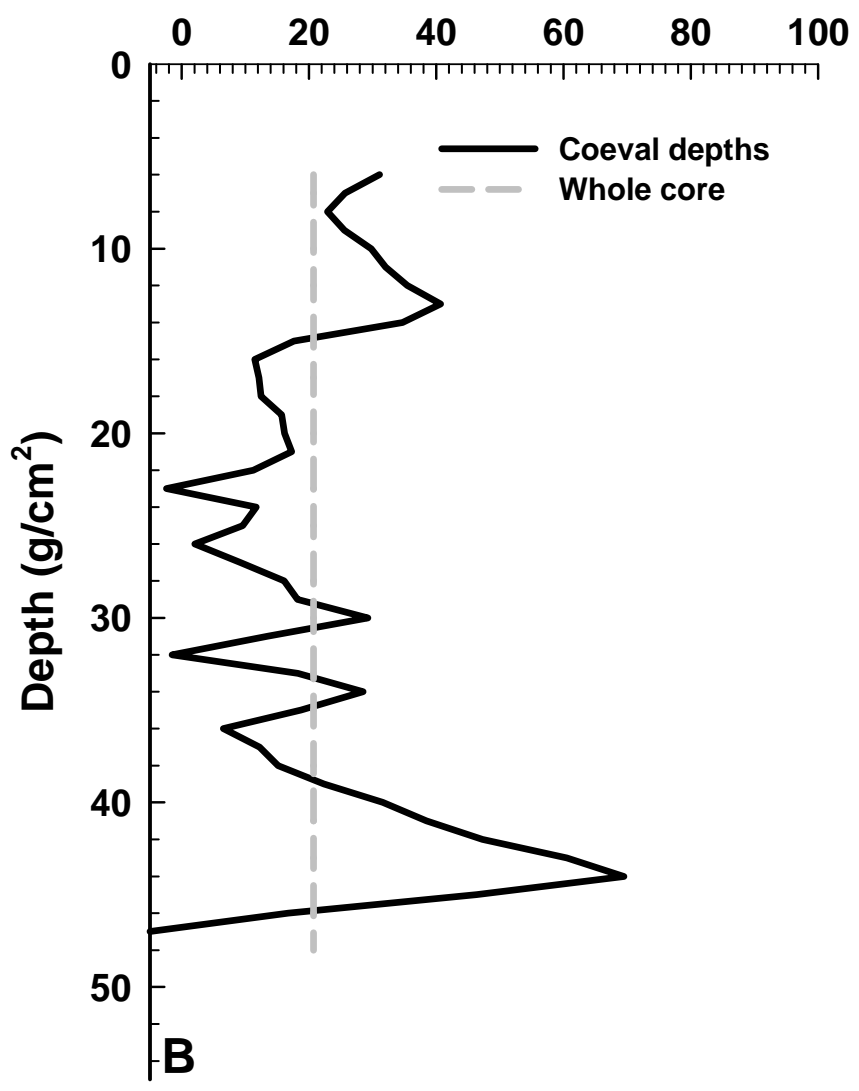

Figure 40. Graph showing vertical profiles of $(A)$ summed molar inventories of $p, p^{\prime}-\mathrm{DDE}+p, p^{\prime}$-DDMU $+p, p^{\prime}$-DDNU, and $(B)$ percent difference in summed molar inventories of cores 124B1-DDT (1992) and 124B1-CH (2003). Core profiles (panel A) are aligned (4-g/ $/ \mathrm{cm}^{2}$ offset). 


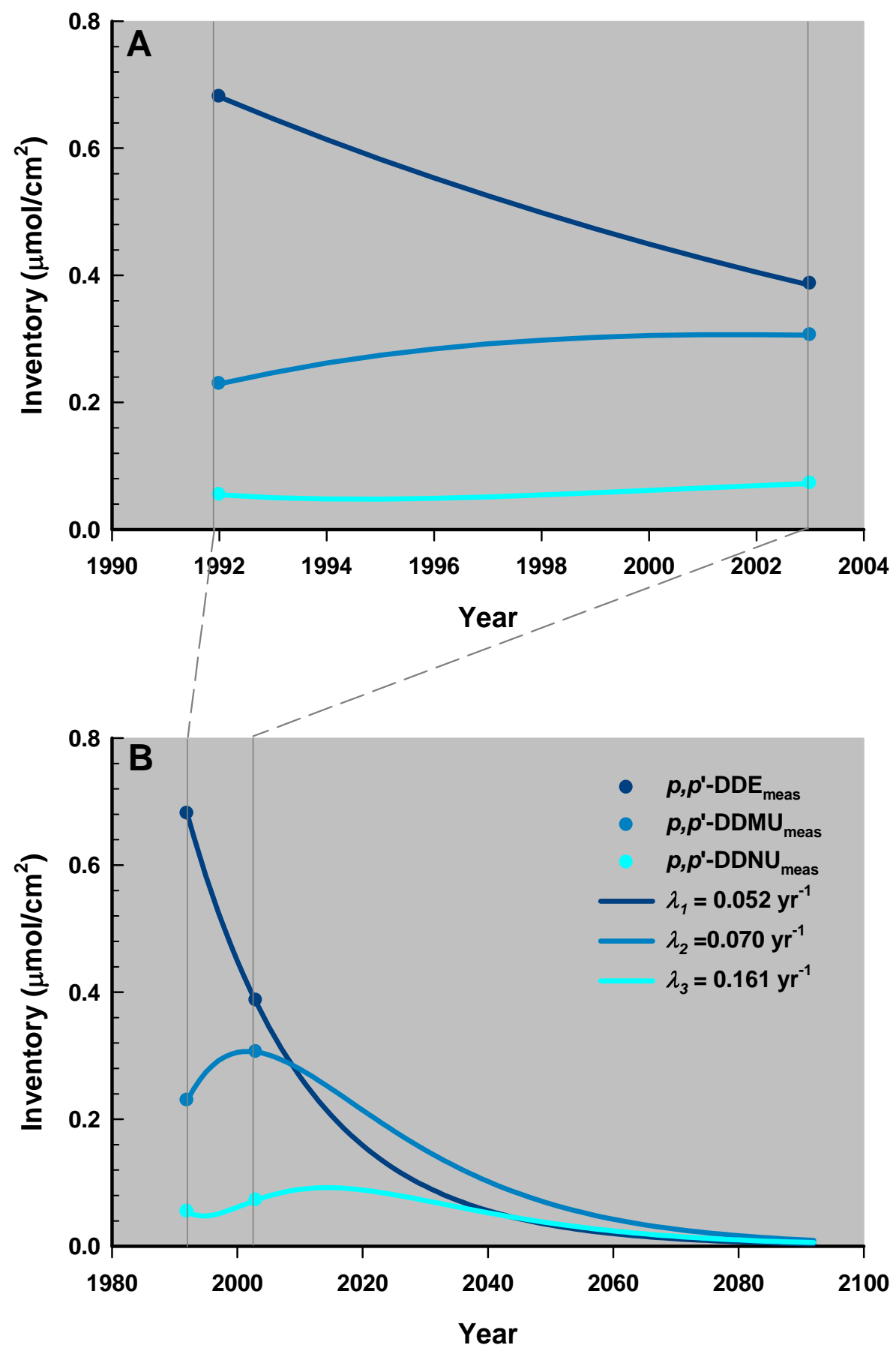

Figure 41. Graph showing trajectories of first-order transformation for $p, p^{\prime}-\mathrm{DDE}, p, p^{\prime}$-DDMU, and $p, p^{\prime}$-DDNU near station 3C: $(A) 1992$ to 2003, $(B) 1992$ to 2092. Solid symbols are measured wholecore molar inventories for cores collected in 1992 and 2003. $\lambda_{n}=$ first-order transformation rates for reaction $\mathrm{n}(1,2,3)$, where $1=\mathrm{DDE} \rightarrow \mathrm{DDMU}, 2=\mathrm{DDMU} \rightarrow \mathrm{DDNU}, 3=\mathrm{DDNU} \rightarrow$ products unknown. 